\title{
STRUCTURAL ANALYSIS OF ORGANOMETALLIC DEPROTONATION AGENTS AND COMPUTATIONAL STUDIES ON FORMALLY HYPERVALENT MOLECULES
}

\author{
Dissertation zur Erlangung des \\ mathematisch-naturwissenschaftlichen Doktorgrades \\ der Georg-August-Universität Göttingen
}

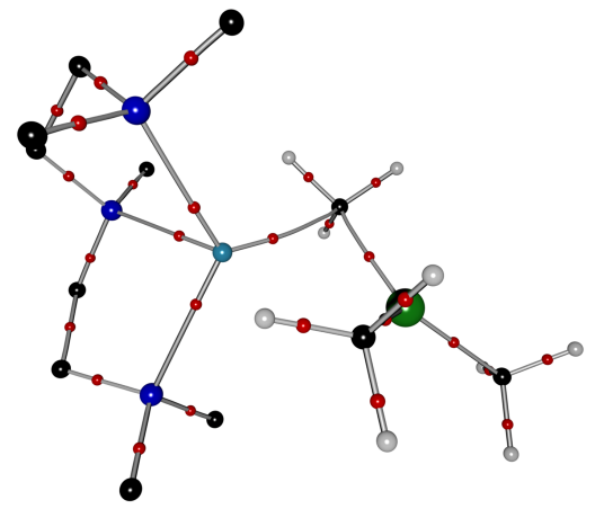

vorgelegt von

Sebastian Merkel aus Schorndorf

Göttingen 2009 
Eingereicht am:

09.12.2009

D7

Referent:

Prof. Dr. D. Stalke

Korreferent:

Prof. Dr. H. W. Roesky

Tag der mündlichen Prüfung:

15.01.2010

Prüfer Anorganische Chemie:

Prof. Dr. D. Stalke

Prüfer Organische Chemie:

Prof. Dr. U. Diederichsen

Prüfer Informatik:

Prof. Dr. W. May

Erweiterte Prüfungskommission:

Prof. Dr. G. Sheldrick

Prof. Dr. G. Eckold 
„Wege entstehen dadurch, dass man sie geht."

Franz Kafka 


\section{List of compounds}

1 2-AnisolLi·pmdeta

2 Scavenger

3 Hydrid

4 Simpkin's amide

$5 \mathrm{Me}_{3} \mathrm{ZnLi}$-diglyme SSIP

$6 \mathrm{Me}_{3} \mathrm{ZnLi}$.pmdeta CIP

$7 \mathrm{Me}_{3} \mathrm{ZnLi} \cdot \mathrm{pmdeta} \mathrm{LiCl}$ SSIP

$8 \mathrm{Me}_{2} \mathrm{Zn}$ theory

$9 \mathrm{Me}_{3} \mathrm{Zn}^{-}$theory

10 Triimid theory

$11 \mathrm{H}_{3} \mathrm{PO}$ theory

$12 \mathrm{SO}_{4}{ }^{2-}$ theory

$13 \mathrm{PO}_{4}{ }^{3-}$ theory

14 Silylene

15 Silylene adduct 


\section{DANKSAGUNG}

Die vorliegende Arbeit wurde in der Zeit von April 2006 bis Dezember 2009 im Arbeitskreis Stalke am Institut für Anorganische Chemie der Universität Göttingen angefertigt.

An allererster Stelle bedanken möchte ich mich bei meinem Chef Professor Dietmar Stalke. Die vielfältigen Möglichkeiten, sowohl präparative Alkalimetallchemie, Kristallographie als auch theoretische Chemie betreiben zu können, sind wohl einmalig und ermöglichen es, sich als ursprünglicher Synthesechemiker auch zusätzliche Fähigkeiten anzueignen und einen weiteren Blickwinkel für die Chemie zu bekommen.

Dem Physiker Dr. Julian Henn habe ich es zu verdanken, dass ich Einblicke in die Welt der Quantenchemie bekommen habe, was meine Arbeit abwechslungsreich gestaltet wie auch wissenschaftlich bereichert hat. Dass er mich auch sportlich beim Joggen und Klettern auf Trab gehalten hat, war ebenfalls wichtig, um nach einem Tag vor dem Computer den Kopf frei zu bekommen.

Herzlich bedanken möchte ich mich auch bei meiner ehemaligen F-Praktikantin Tanja Tatic. Es freut mich, dass ich dich für die lithiumorganische Chemie und den AK Stalke gewinnen konnte, die Zusammenarbeit mit dir hat die Arbeit gleich viel angenehmer gemacht.

Julian Henn und Tanja Tatic danke ich ebenfalls für das Korrekturlesen der vorliegenden Arbeit.

Kathrin Meindl und meinem zweiten Kletterpartner Daniel Stern danke ich für die Unterstützung bei mancher Verzwilligung und Fehlordnung, die die Kristallstrukturanalyse erschwerten.

Meinem Laborpartner und Hobby Fotografen Christian Kling danke ich für die schöne Zeit in unserem Labor, auch wenn es mich später immer mehr in den Computerraum gezogen hat. Besonders die Tips, was man an einem baufälligen Golf 3 alles selber machen kann („Jetzt helfe ich mir selbst“), waren sehr wertvoll.

Unserem Admin Daniel Kratzer und seinem HiWi für die Wartung des Netzwerkes und des Linux Clusters, damit die Quantenrechnungen auch schön parallel liefen.

Ausserdem geht ein Dank an den gesamten Arbeitskreis Stalke für die tolle Arbeitsatmosphäre und den Zusammenhalt.

Bedanken möchte ich mich auch ganz herzlich bei meinen Kooperationspartnern und Kristalllieferanten Dr. Rajendra Ghadwal und Dr. Jiangfeng Li aus dem Arbeitskreis Prof. Roesky. 
Ein grosser Dank geht auch an Herrn Schlote, der für Chemikalien und kontinuierlichen Nachschub an Glasgeräten, die in zahlreichen Kühlschänken verschwanden, sorgte. Desweiteren danke ich den analytischen Abteilungen und der Werkstatt des Institutes, deren Mitarbeiter die Arbeit im Hintergrund leisten, ohne die eine wissenschaftliche Arbeit nicht entstehen könnte.

Der Chemetall AG danke ich für die Bereitstellung zahlreicher lithiumorganischer Basisverbindungen.

Zuletzt möchte ich meinen Eltern und meiner ganzen Familie für die andauernde Unterstützung während des ganzen Studiums danken. 


\section{Content}

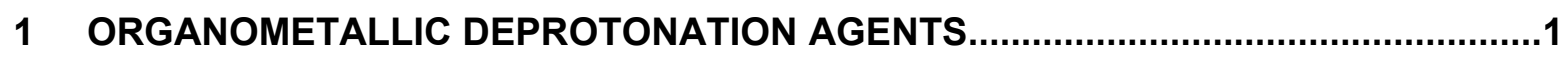

1.1 Monometallic deprotonation agents .....................................................

1.2 Bimetallic deprotonation agents .........................................................

2 STRUCTURAL ANALYSIS OF REACTIVE INTERMEDIATES ...............................13

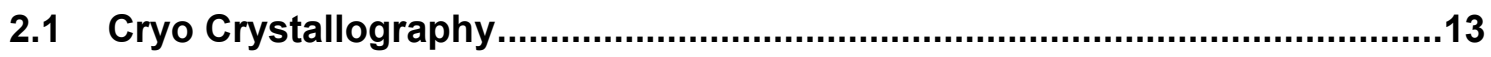

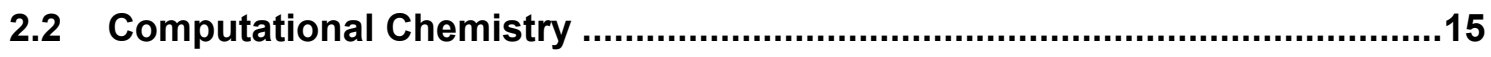

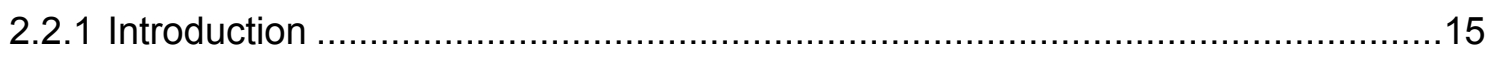

2.2.2 Bridging the gap between $\Psi$ and empirical chemical concepts ......................... 16

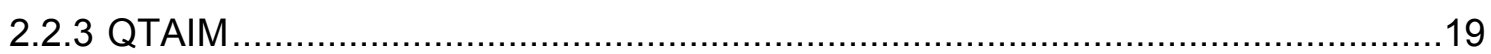

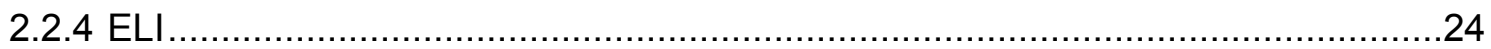

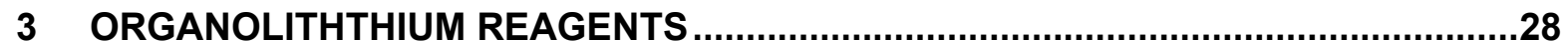

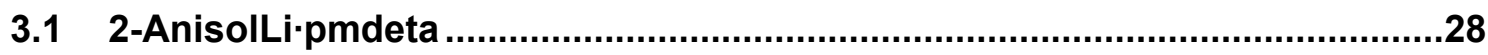

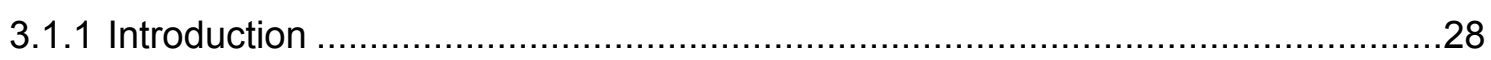

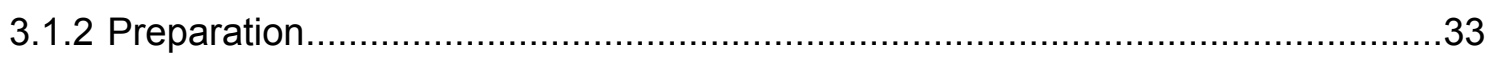

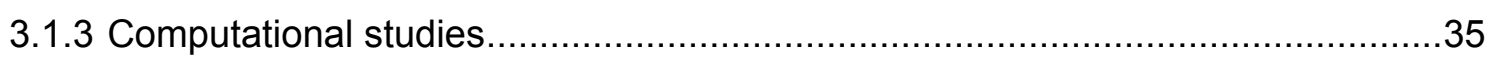

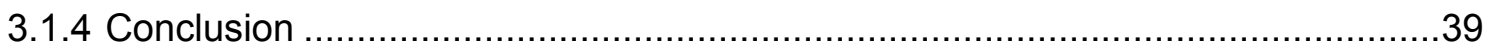

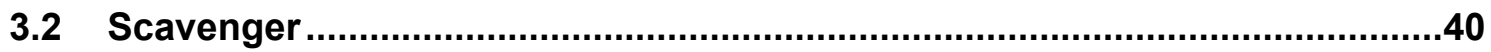

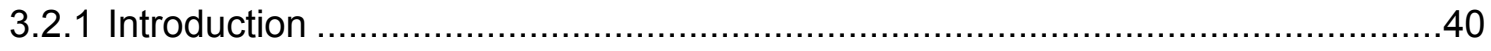

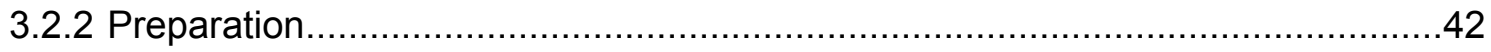

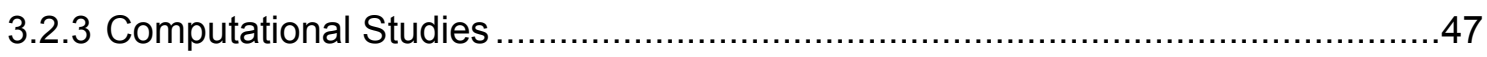

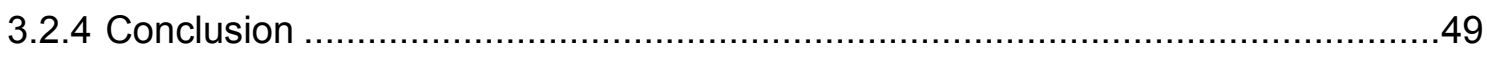

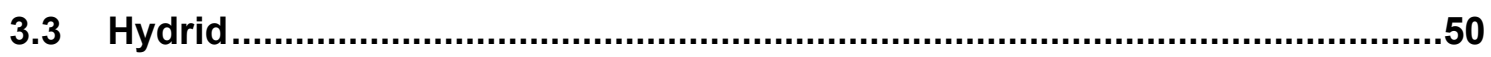

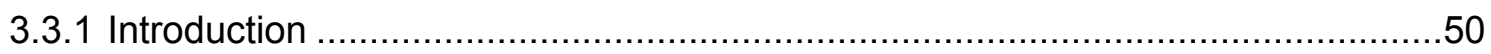

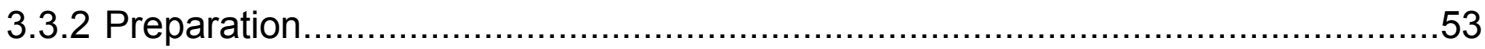

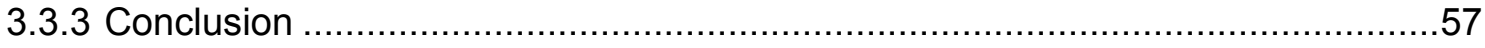

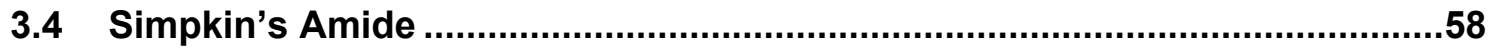

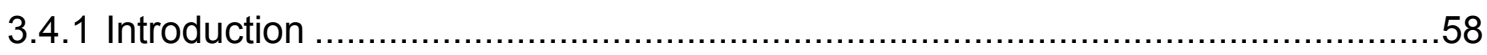

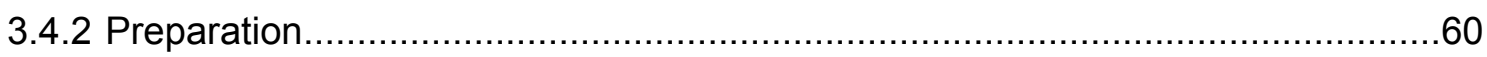

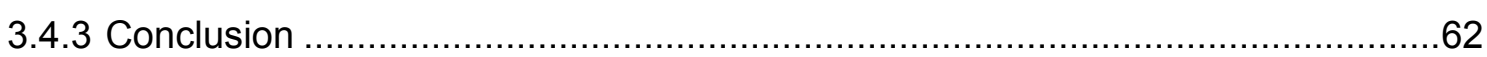




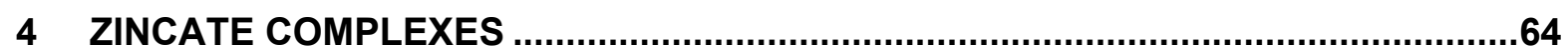

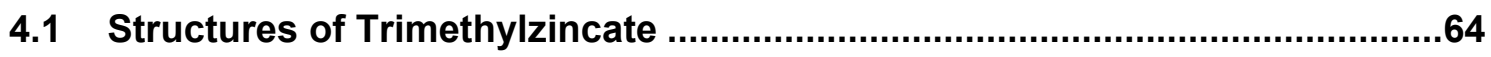

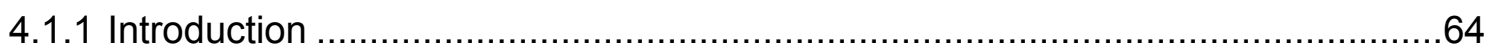

4.1.2 SSIPs and a CIP of parent Trimethylzincate ................................................... 74

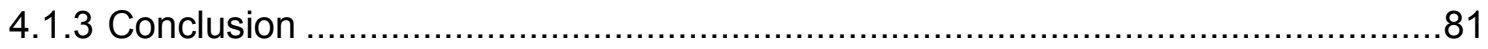

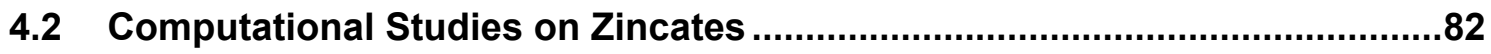

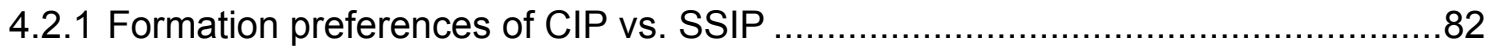

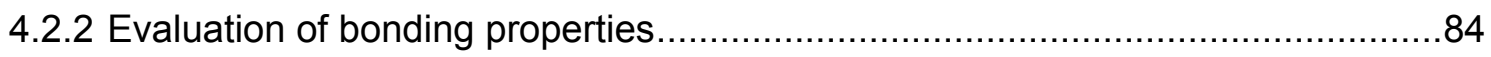

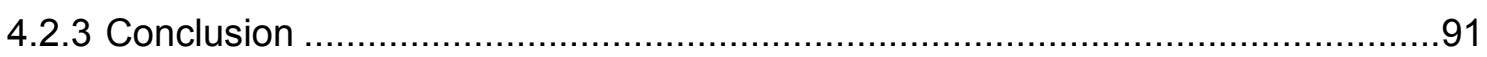

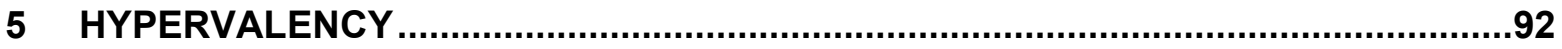

5.1 Evaluation of the bonding situation in formally hypervalent molecules ......92

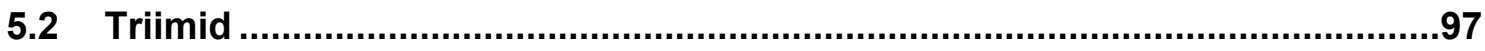

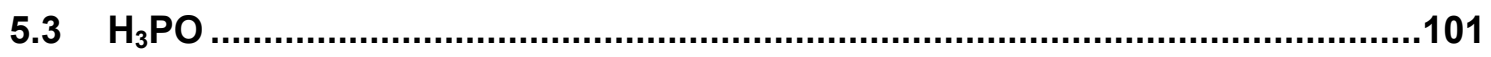

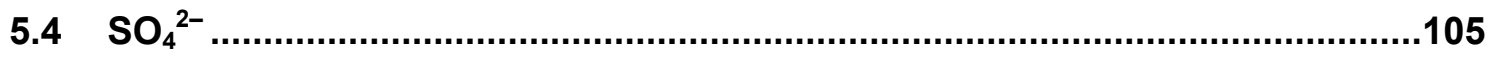

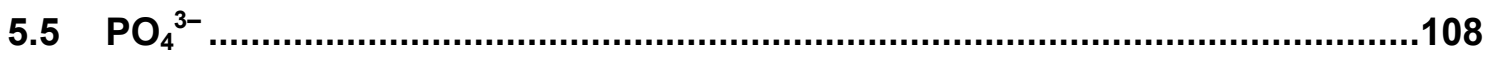

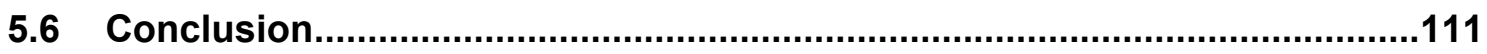

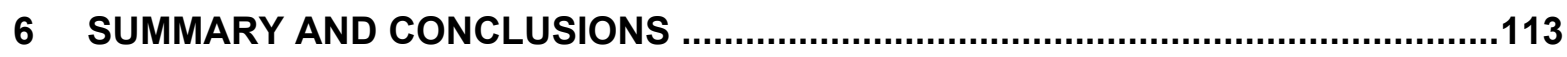

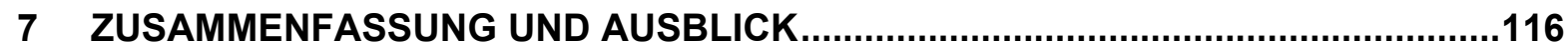

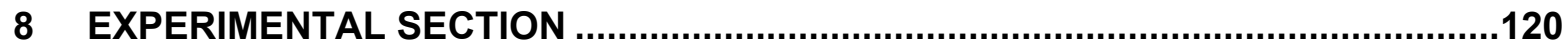

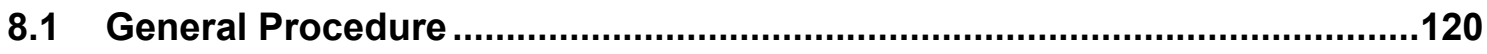

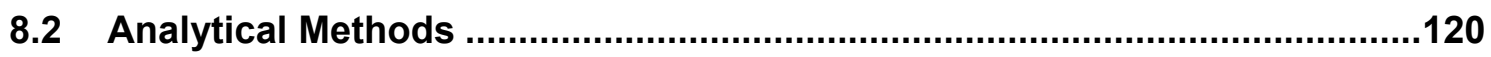

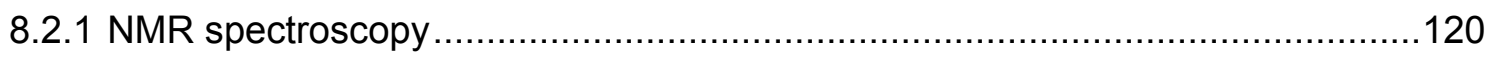

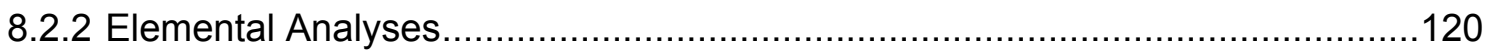

8.3 Syntheses and Characterizations............................................................121

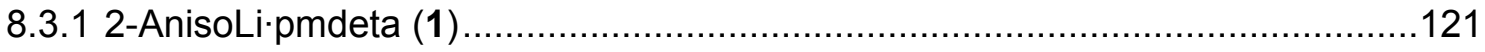

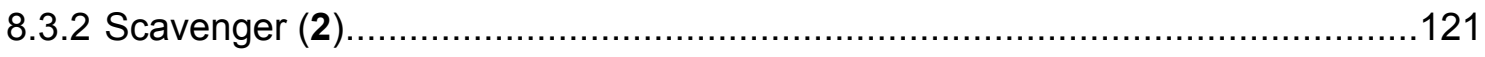

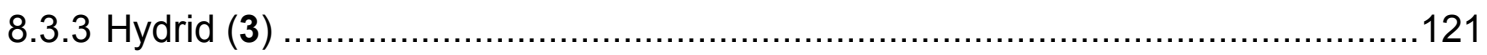

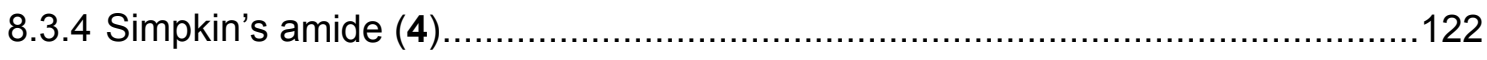

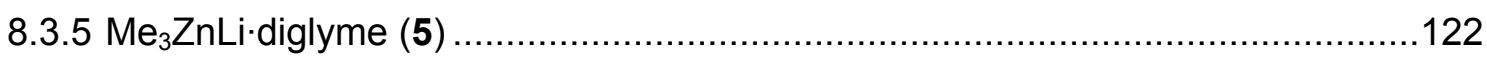

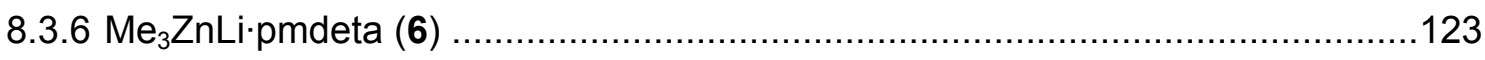




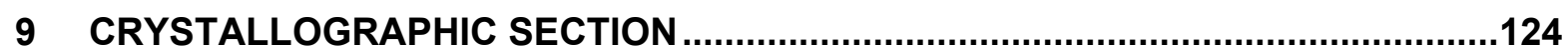

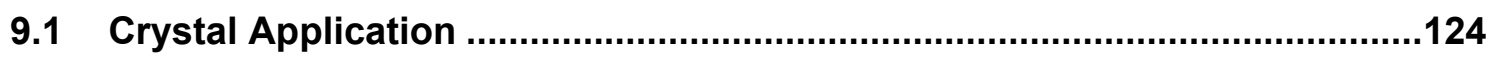

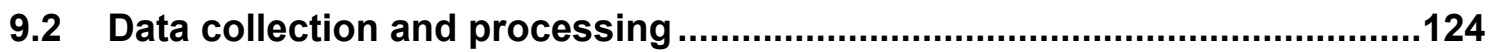

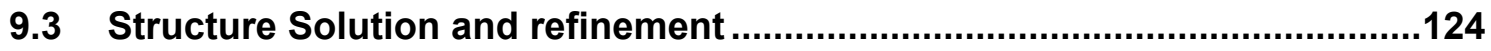

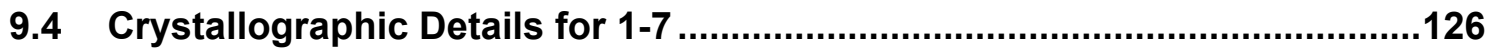

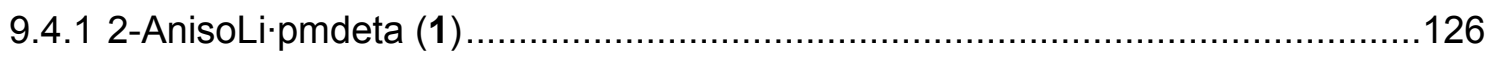

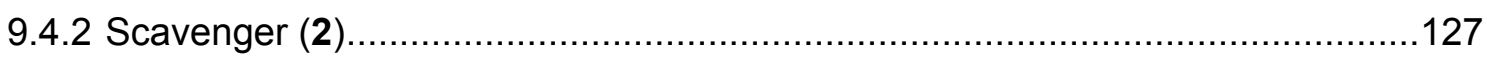

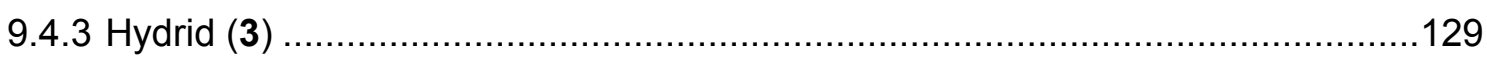

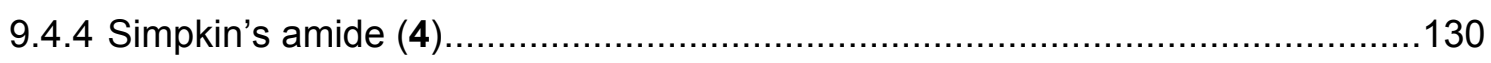

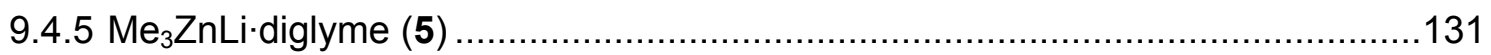

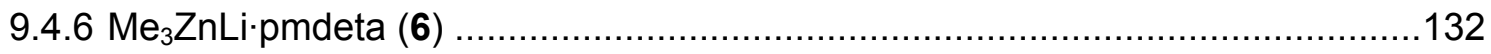

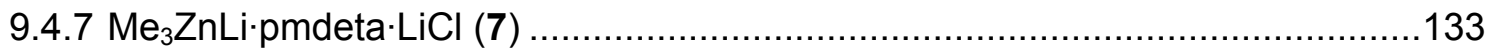

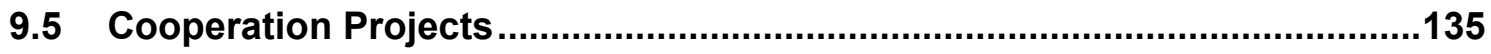

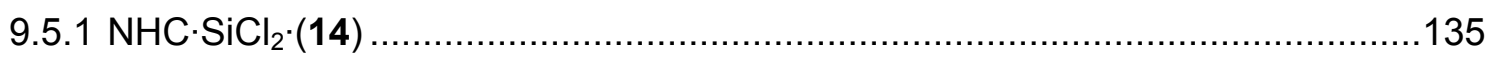

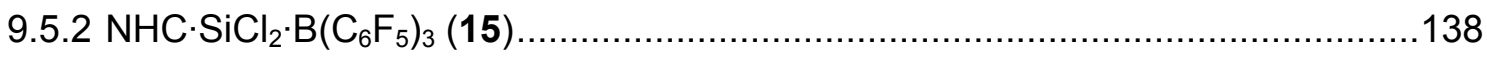

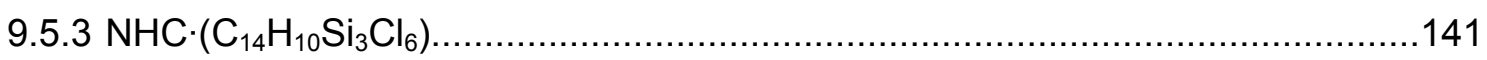

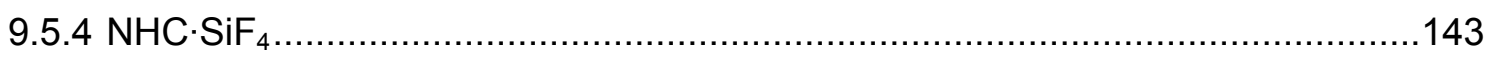

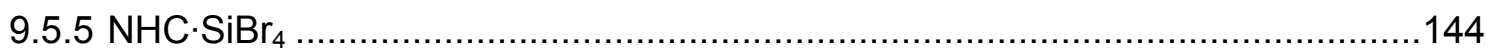

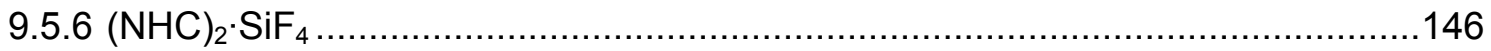

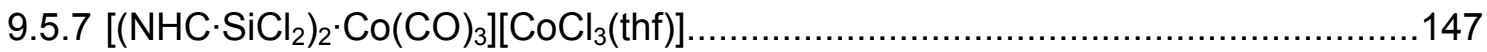

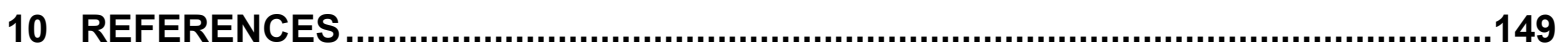




\section{ABbReVAtions}

\begin{tabular}{|c|c|c|c|}
\hline$\AA$ & Ångström & LP & lone pair \\
\hline \multirow[t]{2}{*}{ ADP } & anisotropic displacement & LUMO & lowest unoccupied molecular orbital \\
\hline & parameters & $\max$. & maximal \\
\hline AP & atomic position & M & metal atom \\
\hline au & atomic units & $\mathrm{Me}$ & methyl \\
\hline av. & average & $\min$. & minimal \\
\hline $\mathrm{BCP}$ & bond critical point & MM & multipole model \\
\hline BP & bond path & MO & molecular orbital \\
\hline $\mathrm{Bu}$ & butyl & NBO & Natural bond orbitals \\
\hline $\mathrm{Bz}$ & benzyl & NMR & nuclear magnetic resonance \\
\hline calc. & calculated & $\mathrm{Ph}$ & phenyl \\
\hline DFT & Density Functional Theory & pmdeta & $N, N, N^{\prime}, N^{\prime}, N^{\prime \prime}-$ pentamethyl \\
\hline CIPE & complex induced proximity effect & & diethylenetriamine \\
\hline $\mathrm{CP}$ & critical point & ppm & parts per million \\
\hline CSD & Cambridge structural database & QTAIM & quantum theory of atoms in \\
\hline DoM & Directed ortho metalation & & molecules \\
\hline DOSY & diffusion-ordered spectroscopy & $\mathrm{R}, \mathrm{R}^{\prime}$ & hydrogen atom or alkyl or aryl \\
\hline Diglyme & diethylene glycol dimethyl ether & & group \\
\hline e & electron(s) & RT & room temperature \\
\hline e.g. & for example & sof & site occupation factor \\
\hline ED & electron density & $t$ & tertiary \\
\hline ELF & Electron localization function & thf & tetrahydrofuran \\
\hline ELI & Electron localization indicator & tmeda & tetramethylethylenediamine \\
\hline Eq. & equation & TMS & trimethylsilyl \\
\hline Et & ethyl & tol & toluene \\
\hline et al. & and others & vs. & versus \\
\hline GooF & goodness of fit & VSCC & valence shell charge \\
\hline $\mathrm{h}$ & hour(s) & & concentration \\
\hline HOMO & highest occupied molecular orbital & VSEPR & valence shell electron pair \\
\hline IAM & independent atom model & & repulsion \\
\hline LMO & Localized molecular orbital & & \\
\hline ICKOR & super basic mixtures of & & \\
\hline
\end{tabular}




\section{and potassium alkoxides \\ LIST OF COMPOUNDS}

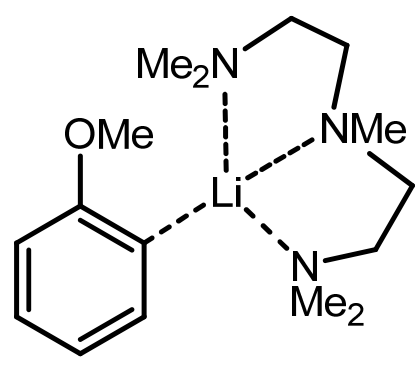

[2-AnisolLi·pmdeta] (1)

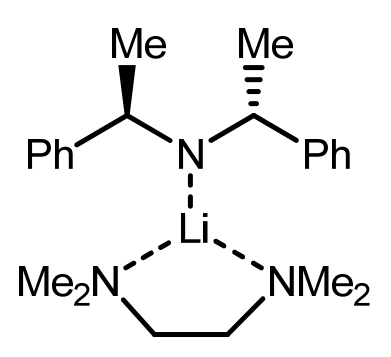

$(R, R)-\left\{[\mathrm{Ph}(\mathrm{Me}) \mathrm{CH}]_{2} \mathrm{NLi} \cdot \mathrm{tmeda}\right\}$ (4)

$\left[\mathrm{Li}_{9}(\mathrm{O})(\mathrm{OtBu})_{5} \mathrm{Me}_{2}\right]_{2}(2)$
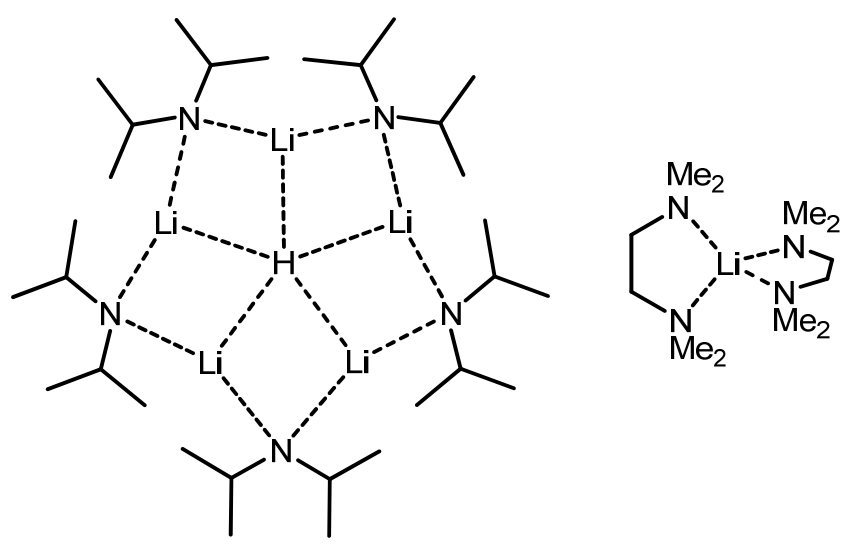

$\left[\left\{(\operatorname{Pr})_{2} \mathrm{NLi}\right\}_{5} \mathrm{H}\right]^{-}\left[(\text {tmeda })_{2} \mathrm{Li}\right]^{+}(3)$

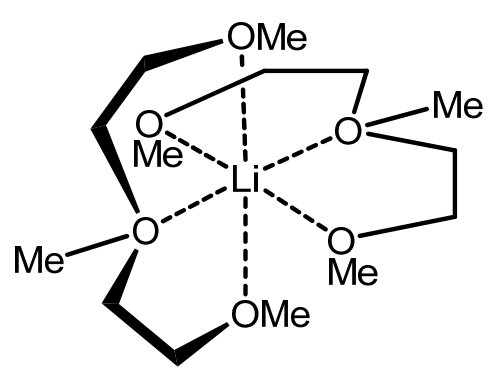

$\mathrm{Me}_{3} \mathrm{ZnLi} \cdot(\text { diglyme })_{2}(\mathbf{5})$<smiles>C[Te](C)C</smiles><smiles>CN1CCN(C)[Te](C)(C)N1C</smiles>

$\mathrm{Me}_{3} \mathrm{ZnLi} \cdot \operatorname{pmdeta}(\mathbf{6})$ 


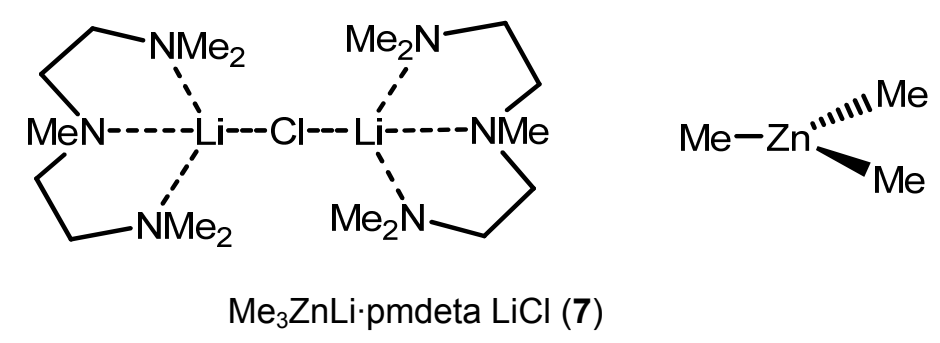<smiles>[1H]N=S(=N)=N</smiles><smiles>O=P</smiles>

$\mathrm{S}(\mathrm{NH})_{3}(10)$

$\mathrm{H}_{3} \mathrm{PO}$ (11)<smiles></smiles><smiles>O=P(=O)([O-])([O-])CO</smiles>

$\mathrm{SO}_{4}{ }^{2-}(12)$

$\mathrm{PO}_{4}{ }^{3-}(13)$<smiles>CCCc1cccc(CCC)c1N1C=CN(c2c(CC)cccc2C(C)C)[C@@H]1[SiH3]</smiles>

$\mathrm{NHC} \cdot \mathrm{SiCl}_{2}(14)$<smiles>CCCc1cccc(CCC)c1N1C=CN(c2c(CCC)cccc2C(C)C)C1C[Si](Cl)(Cl)CP(C(F)(F)F)(C(F)(F)F)(C(F)(F)F)C(F)(F)F</smiles>

$\mathrm{NHC} \cdot \mathrm{SiCl}_{2} \cdot \mathrm{B}\left(\mathrm{C}_{6} \mathrm{~F}_{5}\right)_{3}(\mathbf{1 5})$ 


\section{Organometallic Deprotonation Agents}

\subsection{Monometallic deprotonation agents}

Lithiumorganic compounds are composed of an organic part that is connected to the alkali metal lithium. Lithium itself does not occur in its elemental form because of the high reactivity but in the form of minerals and brines. Among the minerals especially the lithium-rich spodumene played an important role for the extraction of this alkali metal in the past. Today the main sources of lithium are natural brines since their evaporation by solar power is cheaper than the recovering of lithium from ores. One of the world's biggest resources of lithium containing brines is the Salar de Atacama in Chile. The brines are pumped from a depth of $30 \mathrm{~m}$ and processed in a series of evaporation ponds, which span an area over $3 \mathrm{~km}^{2}$ (figure 1-1). ${ }^{[1]}$

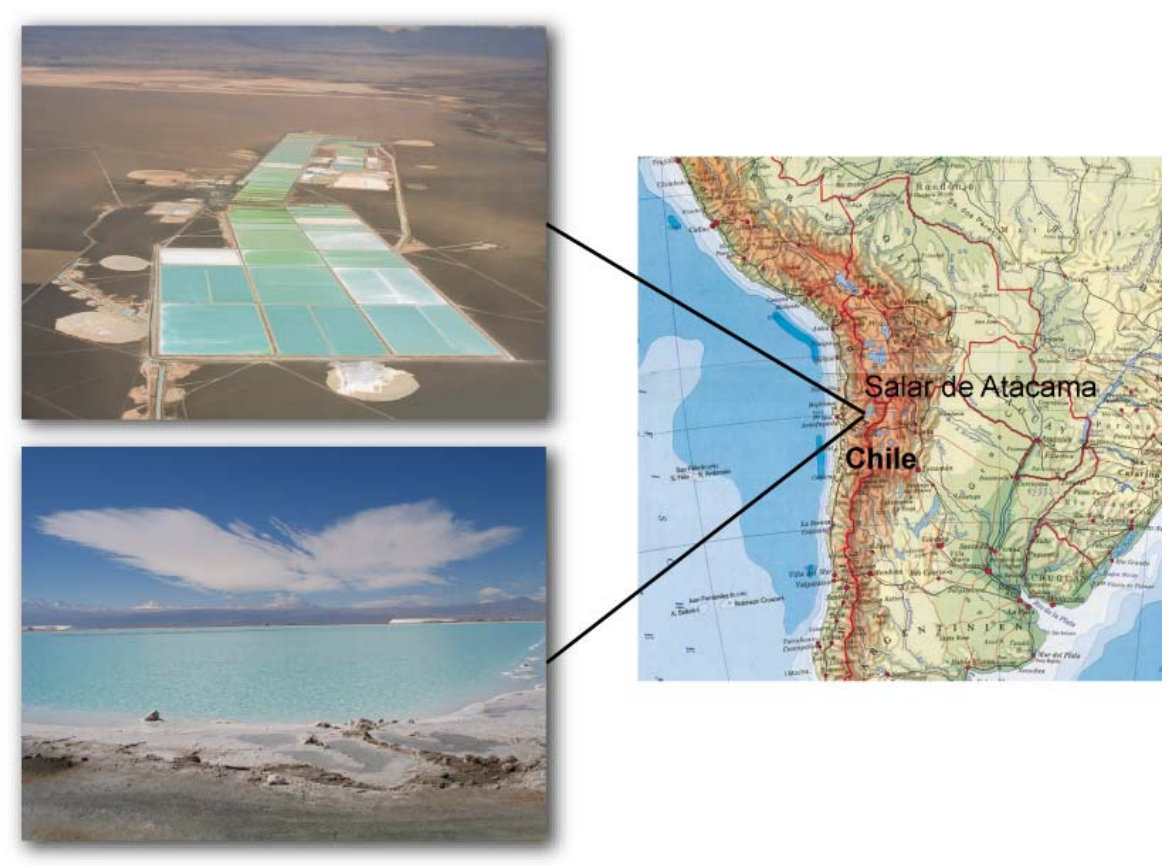

Figure 1-1. Salar de Atacama, Chile.

After 12-18 months this process yields a lithium chloride containing solution (6000 till $60000 \mathrm{ppm}$ ), which can be further worked up. The way to the final lithium organics proceeds via metallic lithium, which is prepared by electrolysis, and is eventually reacted with an organic halide. During this conversion the originally easy to handle raw material becomes a highly reactive organometallic compound, which handling requires sophisticated inert gas techniques. ${ }^{[2]}$

The striking feature that makes these reagents so fascinating is the existence of a carbon-lithium bond. Carbons electronegativity of 2.55 and a half filled valence shell give 
rise to a multitude of compounds, making carbon the atom with the greatest diversity of molecules on earth and the basis of organic chemistry. ${ }^{[3]}$ In organic molecules, carbon forms mainly covalent 2 electron 2 center bonds, which leads to discrete molecules obeying the Lewis octet rule. Even in most organic polymer materials this rule is obeyed, e.g. graphite or diamond. These compounds can easily be described in terms of Lewis structures, in which carbon has a maximum valency of four. When bound to a lithium atom, the behavior of carbon changes dramatically. Since the electronegativity of lithium remarkably smaller than that of carbon, carbon is rendered negative. As the negative charge at carbon is not well stabilized, carbanions are very strong bases, reacting vigorously with moisture and air. Since it is impossible for lithium to obtain an electron octet and because of the high polarity of the bond, organolithiums seek stabilization in forming aggregates. ${ }^{[4]}$ These aggregates are built up according to bonding principles, which exceed the ability of the Lewis model for the description of molecules. The coordination number of carbon can be up to six and the type of bonding has been described as multiple center bonding as well as mostly ionic. ${ }^{[5-8]}$ Furthermore, the degree of aggregation is dependent on solvent, ligands and the presence of co-complexing salts. ${ }^{[9,2,10]}$ Detailed knowledge on the structure of organolithium adducts became available by the developing of cryo techiques in the 1990s making it possible to obtain X-ray data of these highly reactive and pyrophoric reagents. ${ }^{[11]}$ Thereby it was shown that in apolar media the most prominent organolithium $n$-butyllithium is a hexamer. ${ }^{[12]} \mathrm{A}$ donorbase like tmeda is able to break the cluster and produce a dimeric form of $n \mathrm{BuLi}$ (figure 1-2). ${ }^{[13,14]}$
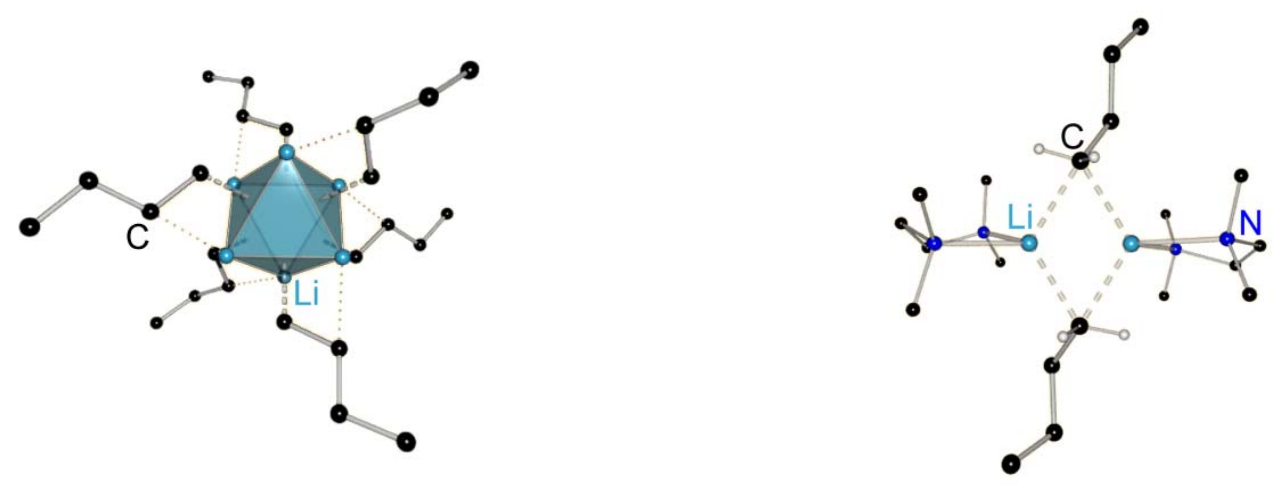

Figure 1-2. Different aggregation grades of $n$ BuLi: As a hexamer in apolar media ${ }^{[12]}$ (left) and a dimer in the presence of tmeda ${ }^{[14]}$ (right).

In some cases solid state structures obtained from single-crystal X-ray analysis reveal just the least soluable or thermodynamically most stable species. In contrast, the situation in solution, where most chemical reactions take place, is more complicated. Here different aggregation states can exist in a rapid superimposed equilibrium. These features of organolithiums make it hard to describe them with simple Lewis structures and write them 
down as if they were single monomers as suggested in many textbooks of organic chemistry. On the other hand, this complexity is responsible for the powerful reactivity in combination with a controllable selectivity of organolithium compounds. Therefore, a deep insight in the structure and bonding modes of these oligomeric aggregates will help to develop new taylor made reagents for the transformation and functionalisation of organic substrates. In the following a few examples shall illustrate the implications due to aggregation behavior in reactions involving organolithium reagents. The first aspect that makes it difficult to postulate a reaction mechanism is the already mentioned equilibrium in solution between different aggregates. Although it has been postulated that reactivity increases with deaggregation, so in principle a monomer should be more reactive than a dimer<tetramer<octamer, it is not always clear, what the active species is. Moreover, newer kinetic studies suggest that dimer based reactions can be even faster than reactions proceeding via a monomer based transition state, since the aggregation energy is not totally forfeited. ${ }^{[15]} A$ second important aspect is the formation of mixed aggregates. ${ }^{[16]}$ In the case of co-complexation with a lithium salt this can enhance the reactivity of the organolithium compound dramatically. ${ }^{[17-20]}$ However, mixed aggregation can also occur between the starting organolithium reagent and a lithiated product, which concentration is rising during the course of reaction. An example for the negative influence of hetero aggregate formation is illustrated in the reaction of $n \mathrm{BuLi}$ with dmaebH (=(R)-[(1-dimethyl-amino)ethylbenzene]) (scheme 1-1). ${ }^{[21]}$ The deprotonation by the established directed ortho metalation $(\mathrm{DoM})^{[22,23]}$ yields the product after quenching with $\mathrm{D}_{2} \mathrm{O}$ in a poor yield of only $50 \%$.
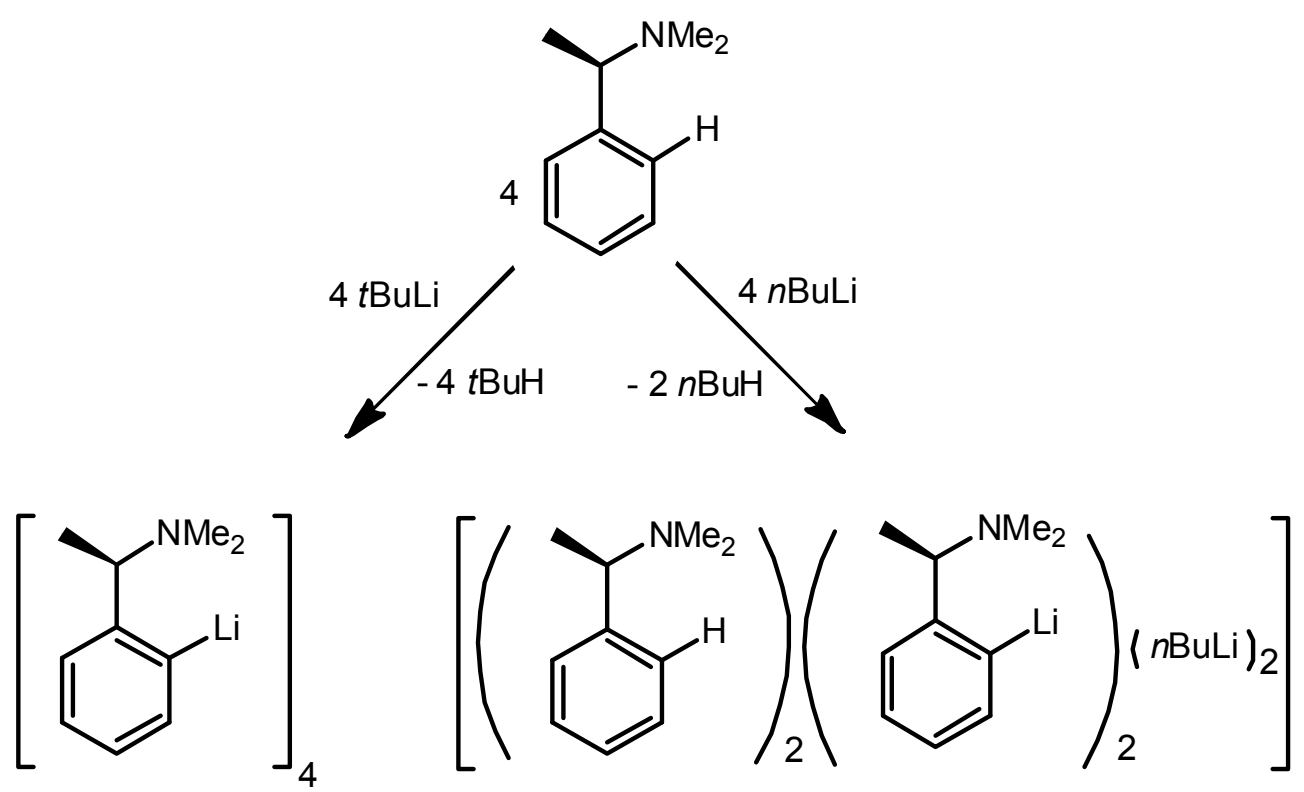

Scheme 1-1. Directed ortho metalation of dmaebH with $t$ BuLi (left) and $n B u L i$ (right). ${ }^{[21]}$

Using tBuLi instead gives complete deprotonation to dmaeLi. Van Koten et al. investigated the reason for this and discovered that not steric effects or the weak nucleophility of the butyl 
anion but the formation of the hetero complex $\left[(\mathrm{dmaebLi})_{2}(n \mathrm{BuLi})_{2}(\mathrm{dmaebH})_{2}\right.$ is responsible for the low outcome with $n \mathrm{BuLi}$. The hetero aggregate is so stable that one equivalent of starting reagent is disabled. ${ }^{[24]}$

Another application area of organolithium bases is the enantioselective deprotonation of organic substrates. Since most bioactive natural products which are of interest for the pharmaceutical industry posses one or more stereogenic centers, asymmetric synthesis of natural products is a key discipline in organic chemistry. While nature performs enantioselective transformations with the help of enzymes, chiral amino acid based catalysts which have been optimized over a long period of time in the evolution, the history of chiral organolithiums is much shorter. Mainly three strategies for the preparation of enantiopure compounds have been developed. The first strategy uses chiral auxiliaries for the induction of enantioselectivity. Chiral auxiliaries like the Schöllkopf-bislactimether ${ }^{[25]}$ or Evan's oxazolidinone ${ }^{[26,27]}$ (scheme 1-2) show a high enantiomeric excess, making them useful tools to introduce and maintain stereocenters in $\alpha$-substituted amino acids or ketones.
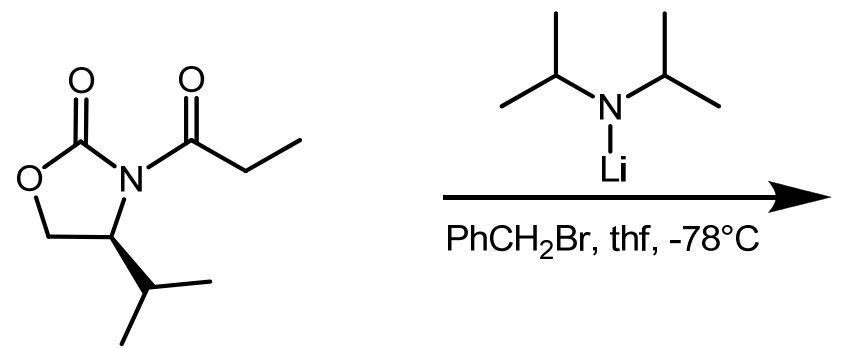

PhCH ${ }_{2} \mathrm{Br}$, thf, $-78^{\circ} \mathrm{C}$

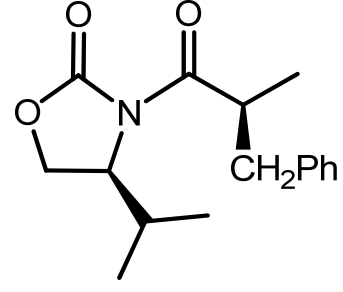

$98 \%$ ee

Scheme 1-2. Evans methodology of the asymmetric alkylation of chiral imide enolates. ${ }^{[28]}$

However, auxiliary-based reactions require the attachment and removal of the auxiliary, two additional steps that decrease the rate of yield. The second strategy is the employment of chiral lithiumamide bases as lithiating agents. ${ }^{[29,30]}$ Unfortunately these suffer from the disadvantage that they must be used in stochiometric amounts.

The third strategy uses chiral ligands as a source of asymmetric induction. Since the chiral additive is employed only in catalytic amounts this method is superior to the previous mentioned strategies. The search for chiral ligands with a broad field of application is an ongoing research area in organolithium chemistry. One of the most successful enatioselective deprotonation system is the combination of a lithium organic and the lupinen alkaloid sparteine (figure 1-5). ${ }^{[31]}$ 

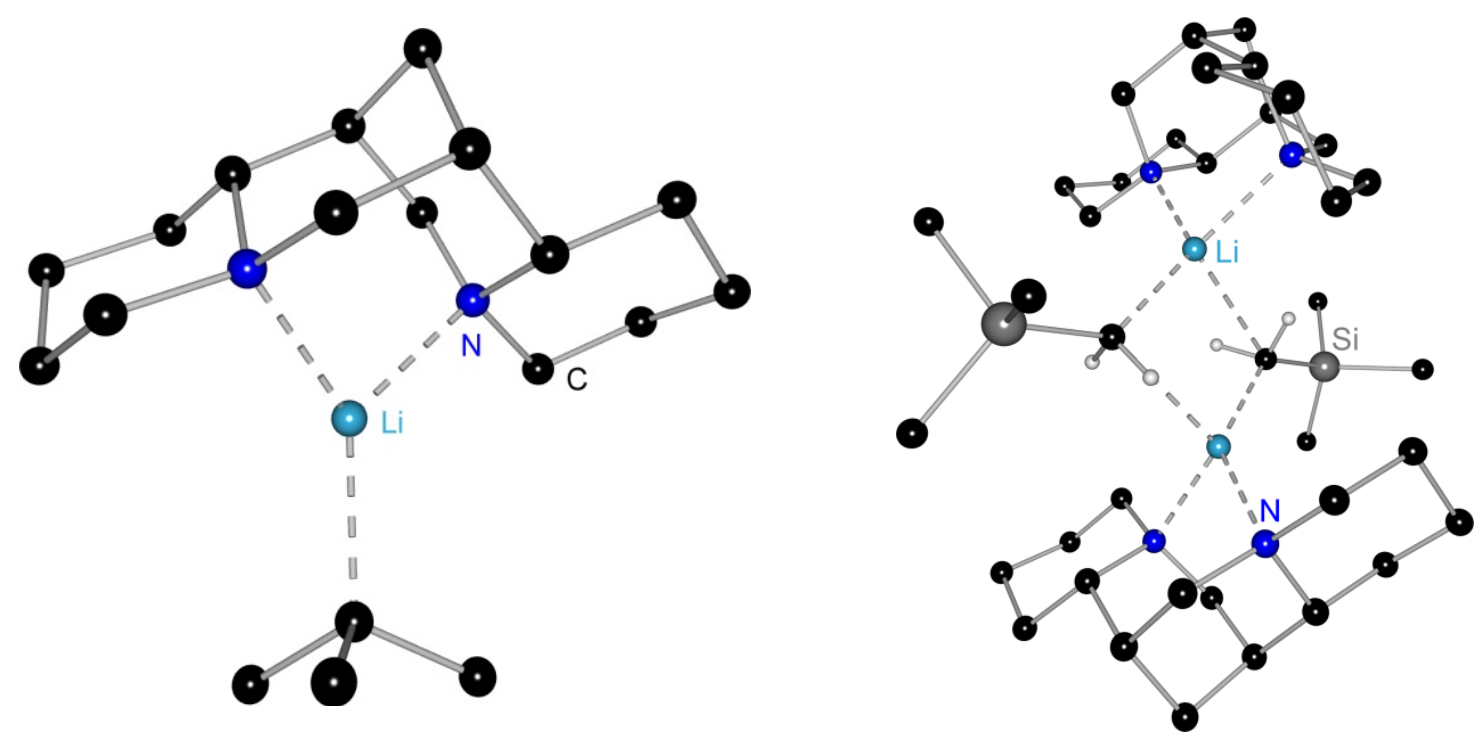

Figure 1-3. Monomeric tBuLi-sparteine ${ }^{[32]}$ and dimeric (TMSMeLi-sparteine) ${ }_{2}{ }^{[33]}$

An example for the specific role of of sparteine is depicted in scheme 1-3. Hoppe et al. used sec-butyllithium and different chiral additives in the stereo selective functionalisation of O-alkyl carbamates. ${ }^{[34]}$ While sparteine is able to induce an enantiomeric excess of $98 \%$, the related (-)-isosparteine fails completely. Other chiral diamines like $(R, R)$-TMCDA show good yields, but the enantiomercic excess is only poor.

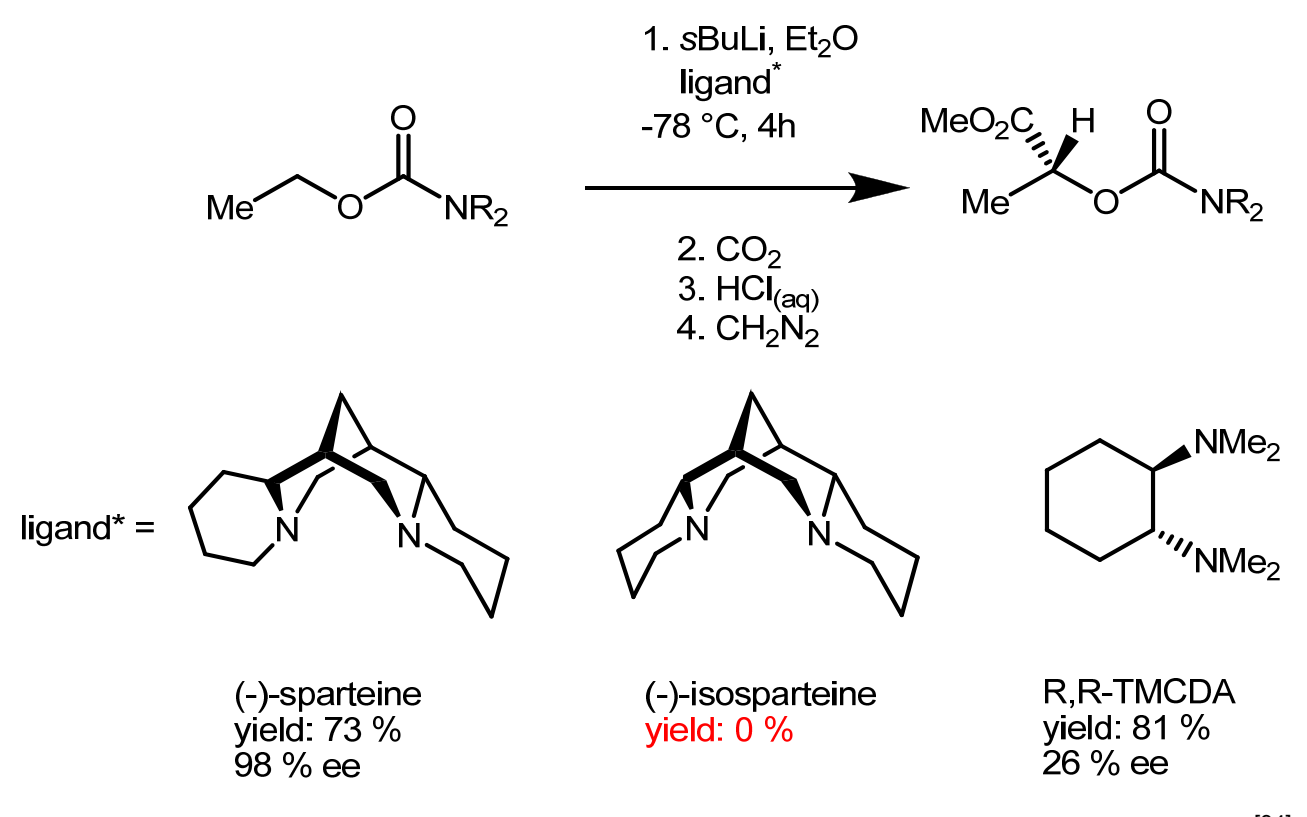

Scheme 1-3. Asymmetric deprotonation of O-alkyl carbamate by sBuLi/sparteine. ${ }^{[34]}$

However, R-Li-sparteine is no universal usable ligand system, since the mechanisms of chiral deprotonation can be quite diverse. Chemists at Schering-Plough found, that in the case of the synthesis of the anti-cancer agent Lonafarnib, sparteine fails completely to induce enantioselectivity, while quinine and hydrochinine $(80-85 \%$ ee) proved to be very 
efficient catalysts for this type of reaction (scheme 1-4). ${ }^{[35]}$ In this special case, the hydroxyl group, which is being deprotonated during the reaction, seems to play a vital role for the enantioselectivity. Furthermore, the enantiomeric excess (ee) can be enhanced by the addition of one equivalent water. This is particularly astonishing since reactions involving moisture and air sensitive organolithiums are normally carried out under inert conditions. Presumably due to the in situ generation of lithium hydroxide aggregation is reduced and a mixed aggregate is formed which has a higher reactivity than the homoleptic aggregate. Interestingly, no effect could be observed, when solid LiOH was added to the reaction. Thus, another ambitious explanation could be that in this rare case water is not deprotonated but rather act as a donor base itself. Indeed, some unusual crystal structures of lithium organics containing coordinated water molecules have been reported. ${ }^{[36,37]}$

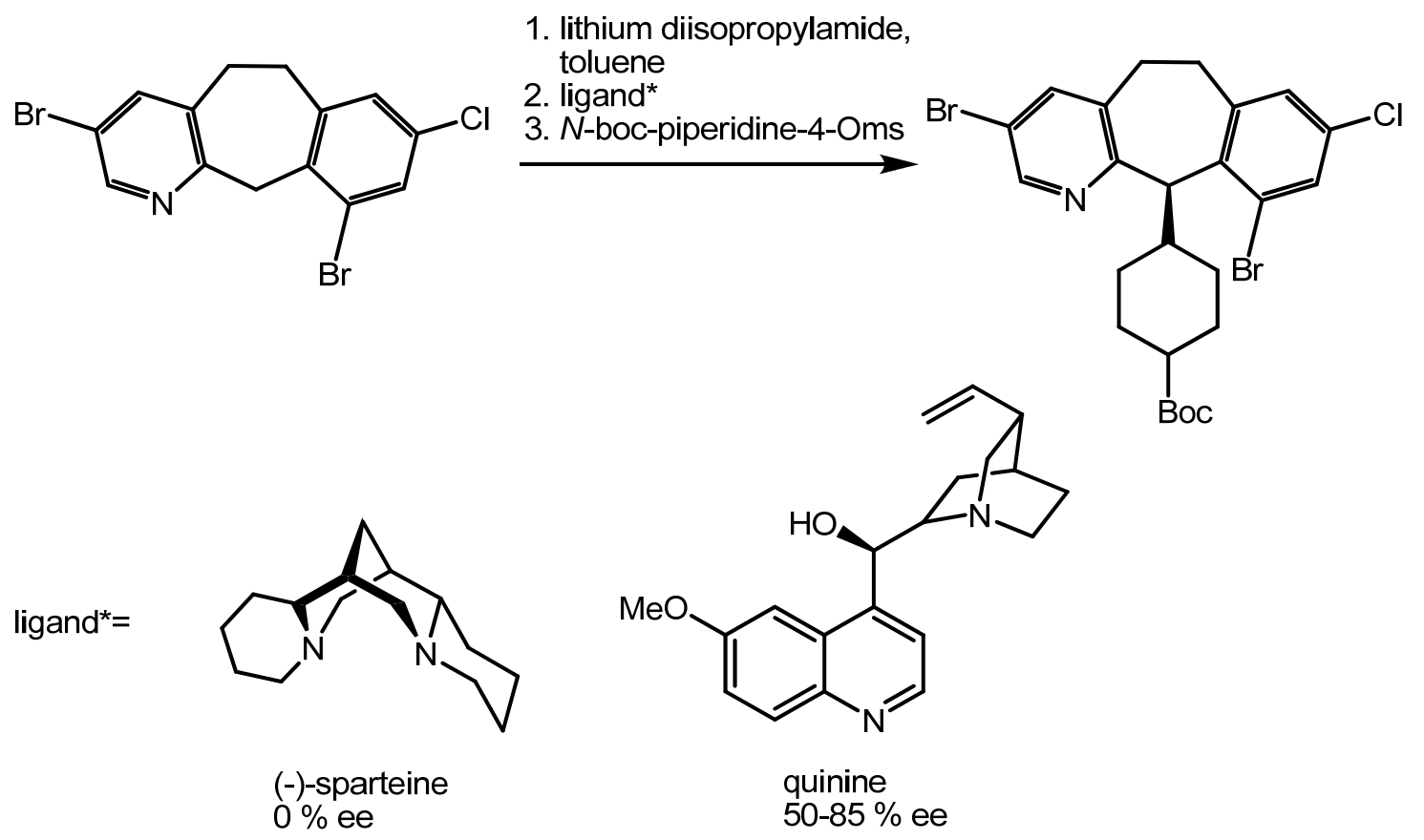

Scheme 1-4. Synthesis of the anti-cancer agent Lonafarnib. ${ }^{[35]}$

A further example, where a mixed species between a chiral lithium alkoxide and lithium acetylide plays an important role, is the asymmetric synthesis of Efavirenz. ${ }^{[38]}$ Efavirenz is an anti-HIV active drug that inhibits the reverse transcriptase. Chemists at Merck have developed a chiral amino alcohol based ligand for the enantioselective addition of $\mathrm{Li}$ acetylide to prochiral ketones. Remarkably, in the synthesis of Efavirenz (scheme 1-5) only Li acetylide addition to the carbonyl function occurs and no abstraction of the acidic nitrogen proton can be detected. 


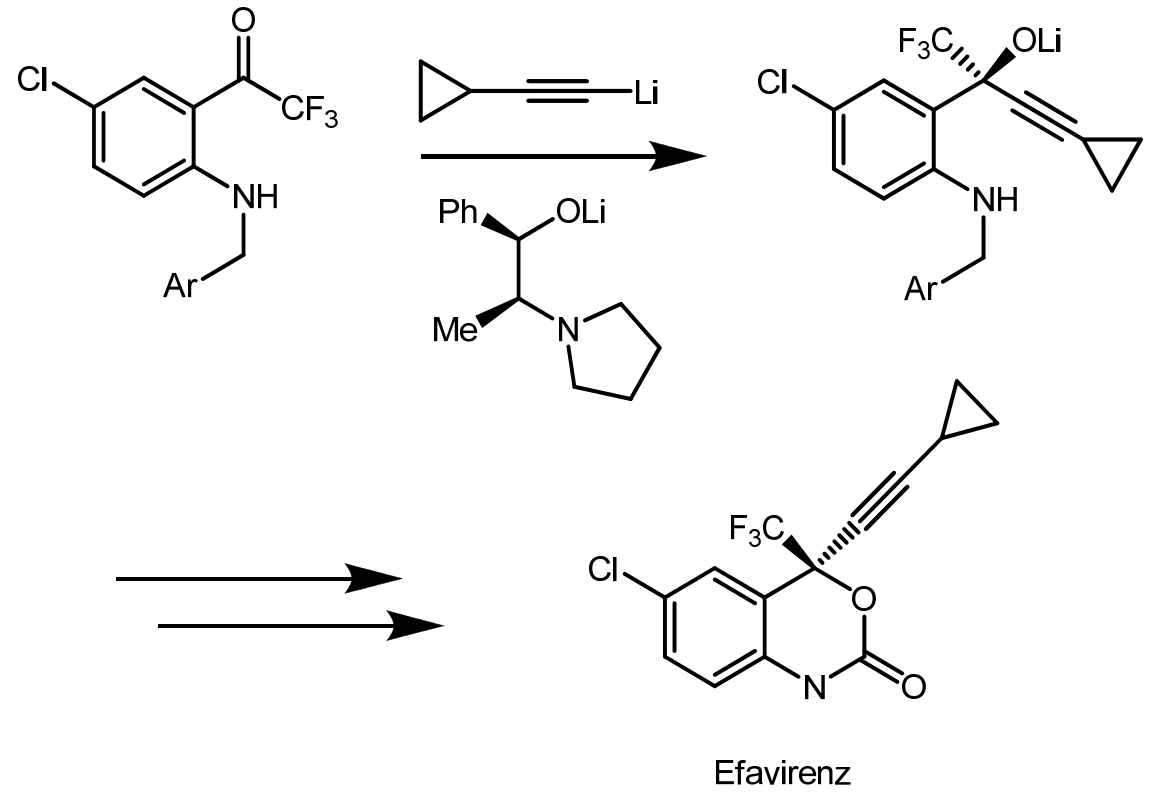

Scheme 1-5. Enantioselective addition of Li acetylide in the synthesis of Efavirenz. ${ }^{[38]}$

In collaboration between Merck chemists and the Collum group the latter were able to deduce the mechanism of the acetylide addition. Both NMR studies in solution and X-ray crystallography support a cubic 2:2 tetramer formed from lithium acetylide and lithium alkoxide to be the reactive species (figure 1-4). ${ }^{[39-41]}$

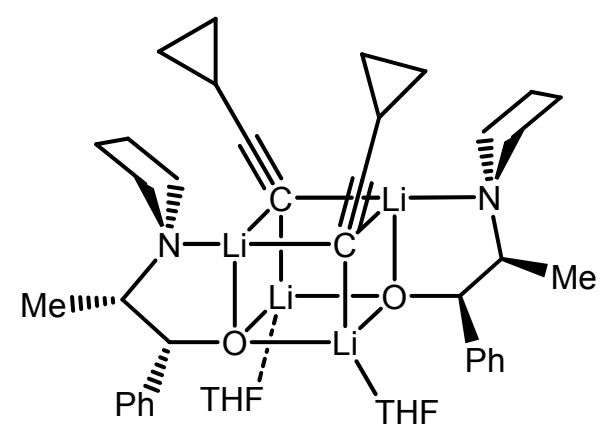

2:2 tetramer

Figure 1-4. Postulated reactive species of the Li acetylide addition in the synthesis of Efavirenz.

While aggregation/deaggregation behavior of organolithium reagents in connection with different donor solvents or ligands is nowadays well understood, the formation principles of hetero aggregates and its implications on reactivity is a field of research that is just starting to emerge. The manifold possibilities of organolithiums to build mixed aggregates make it very difficult to determine the active species and understand the reaction mechanism. Therefore combined solid state and solution studies have to be performed to understand the reaction pathways and hence to be able to develop new reagents. Additional information can be obtained from quantum chemical calculations which can predict the stability of compounds as well as helping in the analysis of bonding in these molecules. The next chapter will 
consequently deal with ate complexes, which are mixed species too, but here two different metals are present in one aggregate, giving rise to a new phenomenon called "synergistic effects".

\subsection{Bimetallic deprotonation agents}

Ate complexes can be regarded as the next step in the evolution of organometallic deprotonation agents. Over the last decade these bimetallic bases have gained much attention since their reactivity outperforms that of classical organolithium reagents in many cases. Ate complexes are metalorganic fragments with formally anionic characteristics. ${ }^{[42]}$ They are formed by the reaction of organometallics possessing a vacant orbital in combination with an unsaturated coordination sphere and a second nucleophilic organometallic (scheme 1-6).

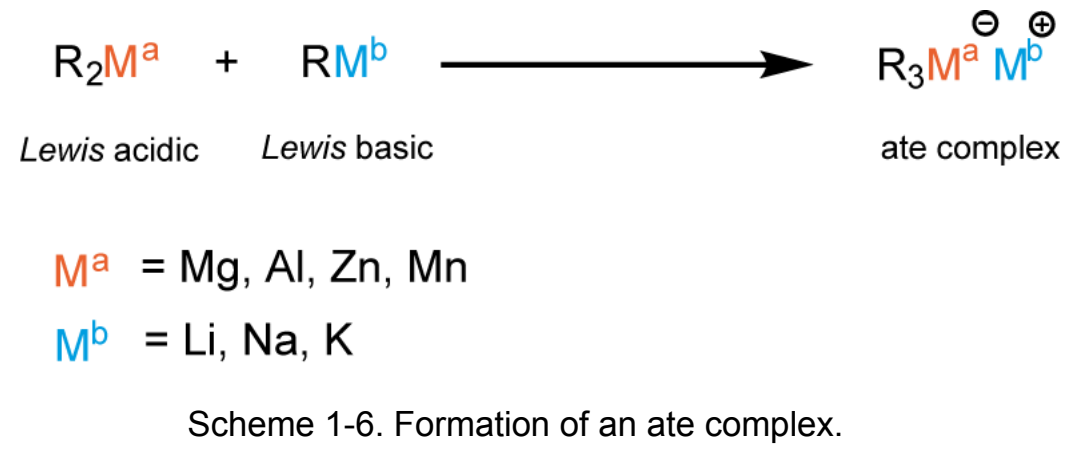

Since it is possible to vary a primary metal center, a secondary metal center and different ligands/solvents independently, the reactivity and selectivity of these reagents can be fine tuned in a way that is not possible for their monometallic congeners (figure 1-5). ${ }^{[43]}$

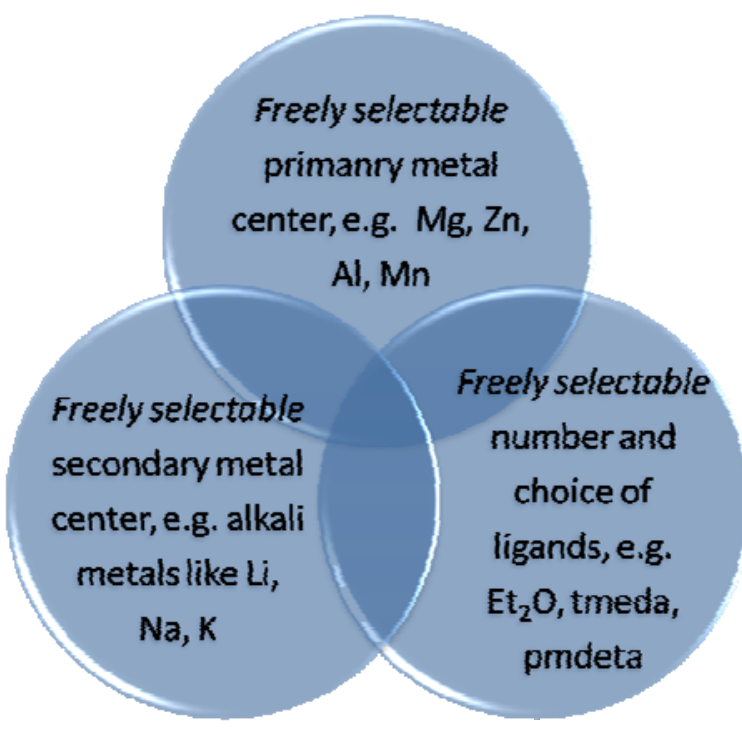

Figure 1-5. The essential building blocks of a modern ate deprotonation agent. 
Ate complexes which play an important role in organic synthesis can be divided in three parts, namely magnesiates, zincates and aluminates. Their application in deprotonation reactions will be outlined by a selection of relevant recent examples, beginning with the magnesiates. A simple but powerful strategy to create them consists in the activation of Grignard reagents by the addition of lithium chloride. The resulting ate complexes of the formula $\left[\mathrm{RMgCl}_{2}\right] \mathrm{Li}$ (scheme 1-7) have been developed by Knochel et al. and proved to achieve a fast bromine-lithium exchange. ${ }^{[44,45]}$ Meanwhile they can be even purchased commercially as so called "Turbo-Grignards" from the Chemetall AG.

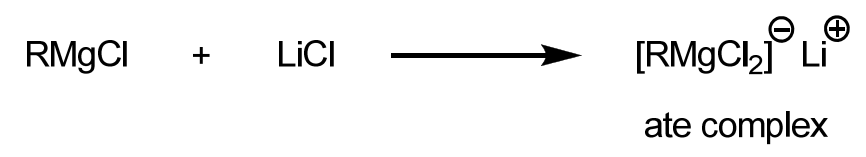

Scheme 1-7. Activation of Grignard reagents with $\mathrm{LiCl}$.

Another useful magnesiate is the mixed amide (tmp) MgCl. $\mathrm{LiCl}$. This agent is able to magnesiate various arenes and hetoroarenes under smooth conditions. Scheme 1-8 exemplifies the high reactivity on the magnesiation of isochinoline. After quenching with iodine the substituted product is obtained with $92 \%$ yield. ${ }^{[46]}$<smiles>c1ccc2cnccc2c1</smiles>

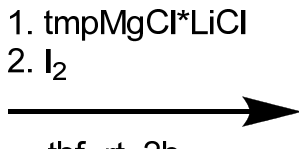

thf, rt, $2 \mathrm{~h}$<smiles>Ic1nccc2ccccc12</smiles>

$92 \%$<smiles>C[C@H](Cl)N1[C@H](C)CCC[C@H]1C</smiles>

Scheme 1-8. Functionalisation of isochinoline with (tmp)MgCl. LiCl.

Howerver, magnesiate complexes can even achieve multiple deprotonations. One of the most impressive demonstrations of the unique reactivity of these reagents consists in the tetrafold metalation of ferrocene. Organolithiums activated by a donor ligand like ether or tmeda are able to deprotonate ferrocene once ${ }^{[47]}$ or twice (once at each ring) respectively, ${ }^{[48]}$ but selective further deprotonations prove to be problematic, since harsher conditions (up to eight equivalents of $n \mathrm{BuLi}$ ) are needed, which leads to a complex reaction mixture containing one till seven-fold metalated ferrocenes. ${ }^{[49]}$ Mulvey et al. where able to show, that neither butylsodium nor dibutylmagnesium alone are able to deprotonate ferrocene, but a mixture of both organometallics generates the $2,2,2^{\prime}, 2$ '-lithiated ferrocene selectively (scheme 1-9). ${ }^{[50]}$ 


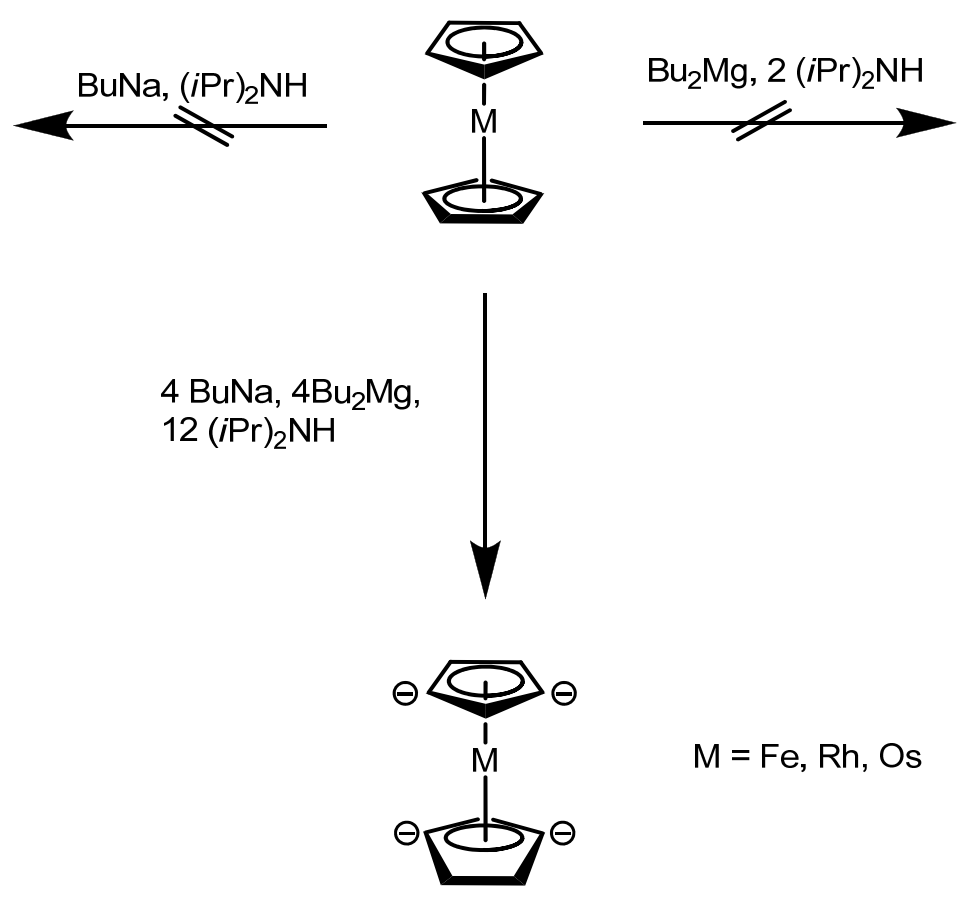

Scheme 1-9. Regioselective tetrafold deprotonation of ferrocene with an ate complex. ${ }^{[50]}$

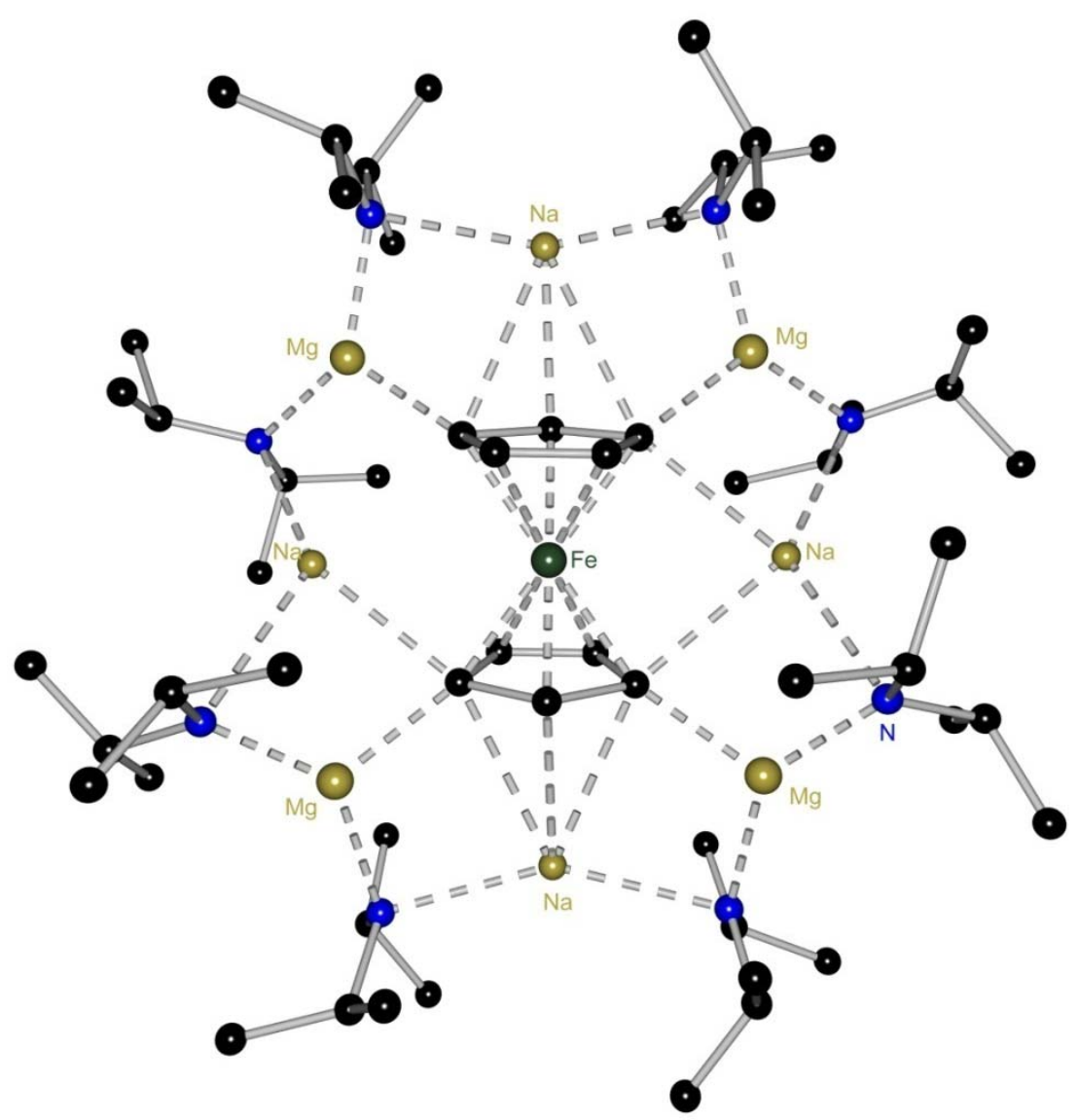

Figure 1-6. Molecular structure of tetrafold metalated ferrocene. ${ }^{[50]}$ 
Since none of the monometallic species alone was able to react with ferrocene, the formation of a highly reactive mixed-metal species must be responsible for this change in reactivity. Mulvey coined the term "alkali metal mediated magnesiation" (AMMM) for this type of reaction, underlying that a synergistic effect between both metals is responsible for this behavior. The X-ray crystal structure gives an explanation how the generation of a fourfold negative anion is possible (figure 1-6). The ferrocene anion is encapsulated by an inverse crown ether, which stabilizes the high negative charge. This inverse crown consists of sodium and magnesium atoms, which are linked by the nitrogen atoms of lithium diisoprolpyl amide.

The second class of ate complexes that has received attention in the last years are aluminium ate bases. These were introduced by Uchiyama et al., who reacted triisobytylaluminium with lithium tmp to obtain $\mathrm{BBu}_{3} \mathrm{Al}(\mathrm{tmp}) \mathrm{Li}$ (scheme 1-10). ${ }^{[51]}$

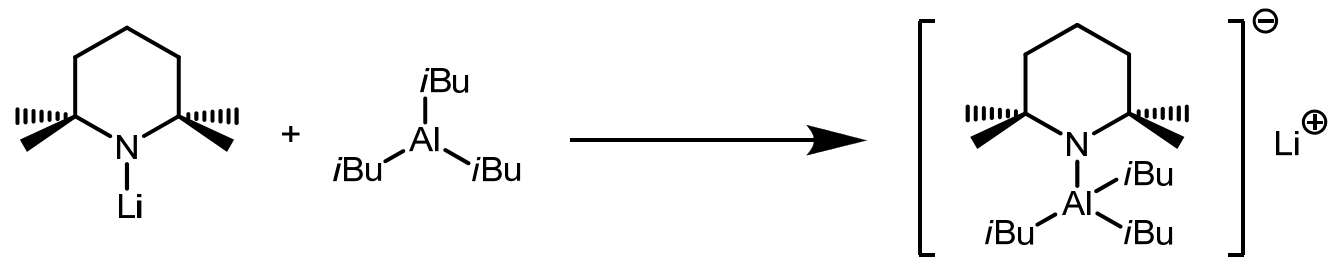

Scheme 1-10. Preparation of the aluminate agent $i \mathrm{Bu}_{3} \mathrm{Al}(\operatorname{tmp}) \mathrm{Li}^{[51]}$

The resulting heteroleptic deprotonation reagent is able to aluminate a range of functionalized aromatics where traditional organolithiums fail because of their incompability with many functional groups. Moreover, a direct alumination of aromatic compounds is not possible but requires a transmetalation step involving organolithium or magnesium reagents, though this leads back to the above mentioned problems with electrophilic functional groups. Scheme 1-11 shows the deprotonation of benzonitrile with $i \mathrm{Bu}_{3} \mathrm{Al}(\mathrm{tmp}) \mathrm{Li}$. Imposingly, the reaction proceeds quantitatively and no side reaction with the cyano group is observed. Mentionable, replacing the $i \mathrm{Bu}$ groups by other alkyl group like methyl, ethyl or tbutyl results in an almost inactive deprotonation reagent.

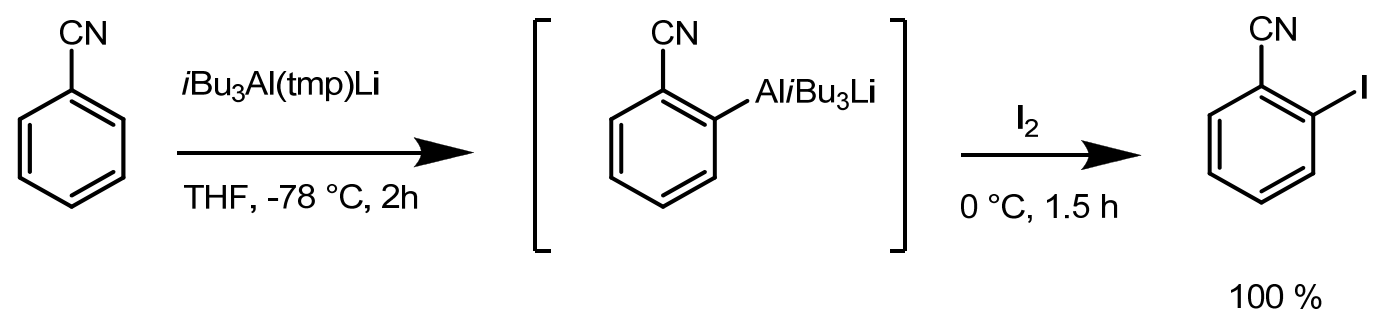

Scheme 1-11. Ortho alumination of benzonitrile with $i \mathrm{Bu}_{3} \mathrm{Al}(\mathrm{tmp}) \mathrm{Li}^{\left[{ }^{[51]}\right.}$

The third important class of ate deprotonation reagents are the zincate complexes. Kondo et al. published in 1999 the mixed amido zincate lithium-di-tert- 
butyl(tetramethylpiperidino)zincate (scheme 1-12) and demonstrated its application as a highly chemoselecive base for directed ortho metalations (DoM). ${ }^{[52]}$

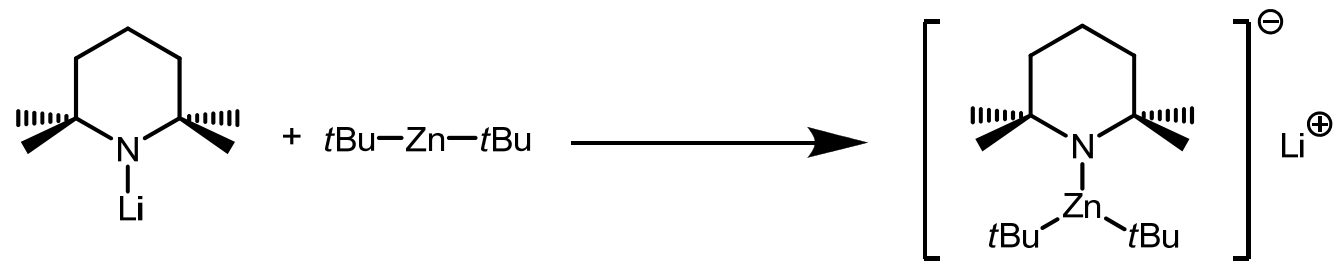

Scheme 1-12. Preparation of the zincate agent $t \mathrm{Bu}_{2} \mathrm{Zn}(\mathrm{tmp}) \mathrm{Li}^{\left[{ }^{[52]}\right.}$

$t \mathrm{Bu}_{2} \mathrm{Zn}(\mathrm{tmp}) \mathrm{Li}$ tolerates even ester groups in substituted benzenes and is able to zincate this substrates regioselective at ambient temperatures (scheme 1-13). ${ }^{[53]}$<smiles>[R]OC(=O)c1ccccc1</smiles>

$\mathrm{R}=\mathrm{Me}, \mathrm{Et}, \mathrm{PPr}, t \mathrm{Bu}$

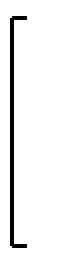

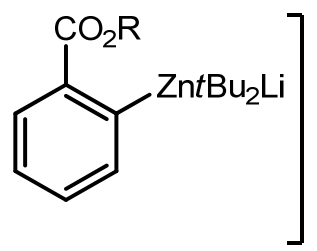

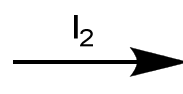

$73-99 \%$

Scheme 1-13. Ortho zincation of alkyl benzoates with $t \mathrm{Bu}_{2} \mathrm{Zn}(\mathrm{tmp}) \mathrm{Li}^{[53]}$

The high tolerance towards functional groups and the smooth reaction conditions make ate reagents in many cases the first choice over traditional monometallic species. Thereby they achieve direct magnesiations, alumination, zincations and recently even manganations ${ }^{[54]}$ of aromatic compounds.

Especially in the synthesis of natural products the employment of protecting groups is reduced, leading to more economic synthetic routes. Furthermore, less side reactions of these highly chemoselective bases provide a higher output and ease product isolation. 


\section{StRUCTURAL ANALYSIS OF REACTIVE INTERMEDIATES}

\subsection{Cryo Crystallography}

Most of the organometallic reagents presented in the previous chapter are thermally unstable compounds which react vigorously when exposed to air or moisture. Therefore they are employed in synthesis under inert conditions. In most cases they are generated in situ and used without further isolation. Instead, they are quenched immediately with an appropriate electrophile and the resulting product is then isolated and characterized. Proposals for the reaction mechanism are mostly based on the obtained product(s), but the structures of the deprotonation agent itself as well as other reactive intermediates along the reaction pathway remain unknown. It seems that these highly reactive organometallics are used more like black box agents.

For a rational approach to optimize these reagents detailed information on the structural properties of reactive intermediates like aggregation state, conformation and steric properties are indispensable. Even more valuable would be precise knowledge on the structural features of the real transition state. Unfortunately, spectroscopic characterization of the transition state is a difficult task since the timescale of the reaction is on femto seconds. Furthermore, quantum mechanics prohibit the observation of the exact transition state as the population at this point reaches zero. Although the reaction pathway is difficult to grasp, nowadays it is possible to "freeze" a reaction at low temperatures and isolate crystals of reactive organometallic intermediates under protective atmosphere. Cryo technologies like the X-Temp 2 system (figure 2-1) developed in the Stalke group make it possible to mount these air, moisture and temperature sensitive crystals on an X-ray diffractometer and collect ultra-low temperature data at $100 \mathrm{~K} .^{[11]}$
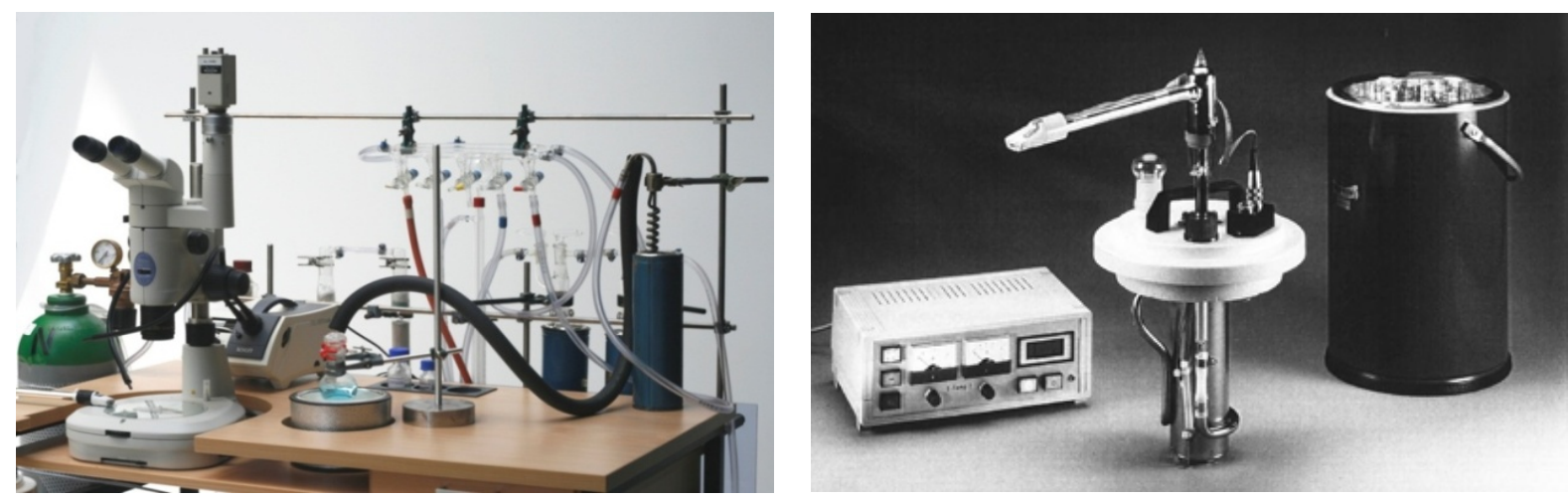

Figure 2-1. Crystal mounting table (left) and X-Temp 2 system (right). 
In this way it is possible to get an insight into the structure and aggregation state of reactive organometallic intermediates, which is crucial for the understanding of reactivity patterns. This methodology was first applied to the alkyllithiums $n$-butyllithium and tertbutyllithium. ${ }^{[12]}$ Both compounds are pyrophoric and in addition $n \mathrm{BuLi}$ is an oil at room temperature. Without the application of cryogenic techniques the elucidation of the crystal structures of these frequently used organolithium reagents would not have been possible.

But identifying the active species is only the first step. For the postulation of the reaction mechanism it is necessary to locate active sites in the molecule e.g. for electrophilic attack and to evaluate the strength of bonds to be broken due to the course of reaction. According to Hohenberg and Kohn this information is contained in the distribution of the electron density (ED). ${ }^{[55]}$ In theory, the ED is experimentally available from X-ray data. However, in standard structure determinations the Independent Atom Model (IAM) is applied. Here, the total electron density is modeled as the superposition of spherical atomic densities. Any deformation of the ED due to bonding phenomena is ignored. Therefore, the IAM model only gives information on atomic positions and anisotropic displacement parameters. A discussion of bond properties is only possible in terms of bond distances and angles. The more advanced multipole model of Hansen and Coppens includes non-spherical density functions. ${ }^{[56]}$ Hereby the atomic ED $\rho(\mathbf{r})$ is constructed from three components, namely a term for the spherical core and one for the spherical valence densities. A third term is accounting for the deformation density. This aspherical density model is able to describe the electron density in bonding regions accurately making it possible to analyze the bond characteristics in a molecule. However, the requirements for this procedure on the crystal quality are extremely high, making it difficult if not impossible for disordered or twinned structures to obtain an experimental charge density. A rare example of a successful multipole refinement of a twinned structure has been published in 2009 by Stalke and coworkers. ${ }^{[57]}$ On the other hand, the electron density can easily be determined by quantum chemical calculations. Ideally, both methods are applied at the same time, making it possible to evaluate experimental results against quantum theoretical calculations. Unfortunately, the thermodynamically unstable organometallics which are the topic of this thesis tended to be hard to crystallize and the crystal quality was not sufficient for experimental charge density studies. Therefore, in this work the structural information from the X-ray experiments (IAM model) was used as a starting point for subsequent computational studies to elucidate bonding properties. 


\subsection{Computational Chemistry}

\subsubsection{Introduction}

Ever since the first theoretical calculations of Heitler and London in 1927, ${ }^{[55]}$ computational chemistry has emerged as a widely used and powerful tool for the calculation of molecular properties. Nowadays, the majority of publications in high ranked journals include experimental results accompanied by quantum chemical studies for verification and comparison. Moreover, computational chemistry can guide experimental chemists by the prediction of yet undiscovered new fascinating molecules. The following section is intended to give a brief history over the basic ideas of quantum mechanics, which today are implemented in popular quantum chemical computer programs like Gaussian ${ }^{[58]}$ or Turbomole..$^{[59-61]}$

Everything started with the Schrödinger equation (Eq 2-1). This equation contains only the fundamental constants such as Planck's constant $\hbar$ the mass of the electron $m_{e}$ and the fundamental charge e. Computational methods that solve the Schrödinger equation without reference to experimental data are termed ab initio.

$$
\left[-\frac{\hbar^{2}}{2 m_{\theta}} \nabla^{2}-\frac{\theta^{2}}{4 \pi \varepsilon_{\theta} \gamma}\right] \Psi=E \psi
$$

Eq 2-1. Schrödinger equation for the hydrogen atom.

A major drawback is that already the $\mathrm{H}_{2}^{+}$ion is the biggest chemically interesting system for which this equation can be solved analytically. This is due to the fact that in manyelectron problems electron-electron repulsion has to be taken into account. In order to make calculations on molecules of practical interests possible, a chain of approximations to facilitate the solution of the Schrödinger equation have been developed. ${ }^{[62]}$ The first step is the introduction of the Born-Oppenheimer approximation, which treats the electrons as if they were moving in a field of fixed nuclei. This is reasonable since nuclei are much heavier than electrons and hence move much slower. In contrast the electrons will react immediately to any change of nuclear positions. Therefore, the electronic part can be solved for different molecular geometries which leads to the potential energy surface (PES). The second step is to find appropriate wavefunctions that are solutions of the Schrödinger equation. The first attempt was made by Hartree, who expressed the $\mathrm{N}$-electron wavefunction as the product of $N$ one-electron wavefunctions. Each of these functions is composed of a part describing the spatial distribution of the electron multiplied by its spin. The product of these spin orbitals is called Hartree-Product and bypasses the problems when solving many electron wavefunctions. However, electron-electron repulsion is included only in an average way and 
the equations have to be solved iteratively until a self consistent field (SCF) is obtained. Eventually, the formulation of $\Psi$ as a determinant ensures that the wavefunction is antisymmetric with respect to the interchange of two electrons. In this way, the Pauli exclusion principle is included in the Hartree-Fock (HF) theory. However, the movement of electrons with opposite spin still remains uncorrelated. In order to perform calculations, a representation of the unknown wavefunction is needed. Thus, the next approximation consists in expanding the solutions of the HF equations as linear combinations of basis functions, which form a so called basis set. Although Slater type functions mimic the true shape of the electron distribution much better than Gaussian type functions, the latter are used in most cases for computational reasons. The correct shape of the molecular orbitals (MOs) is therefore achieved by the combination of a set of overlapping Gaussians. These are used in the Roothaan-Hall equations, which are solved variationally, until the lowest possible energy is reached. Larger basis sets afford more flexibility and will lower the energy until a limit is reached, which is called the Hartree Fock limit $E_{o}$. Since electron motion is not correlated within HF theory, $\mathrm{E}_{0}$ will always be higher than the exact energy. The difference between those two energies is the correlation energy. To target the problem of electronelectron correlation, since the late 1980' different post-HF methods have been developed. These include the Configuration Interaction $(\mathrm{Cl})$ scheme which uses additional determinants of excited states or the Møller-Plesset pertubation theory. Unfortunately, these methods are computationally very demanding. An alternative provides the density functional theory (DFT). ${ }^{[63]}$ The basis of DFT is the Hohenberg-Kohn theorem, which states, that all properties of a molecular system are available from the electron density itself. The goal is to find a functional that connects the density with the molecular properties. One of the most popular functionals is the hybrid functional B3LYP. ${ }^{[64-66]}$ Only since the development of DFT methods it is possible to compute big molecules with high accuracy in appropriate time. In 1998 the impact of DFT on computational chemistry was awarded with the Nobel Prize in Chemistry to Walter Kohn. ${ }^{[67]}$ As DFT provides the best trade-off between performance and accuracy, it was chosen for all calculations in this thesis.

\subsubsection{Bridging the gap between $\Psi$ and empirical chemical concepts}

Generally, the quantum chemical studies started with the coordinates of the crystal geometry as a starting point. After a gas phase optimization to exclude crystal packing effects, the wavefunction $\Psi$ describing the molecule was obtained. At this stage it is a somewhat inconvenient situation for chemists that $\Psi$, being a multidimensional function, is not very descriptive. Although the postulates of quantum mechanics state that $\Psi$ contains all 
molecular information which is accessible via the application of operators, $\Psi$ itself can't be obtained experimentally and its connection to popular chemical concepts is not always apparent. By having a look at the Slater determinant (figure 2-2), this dilemma can be clarified.

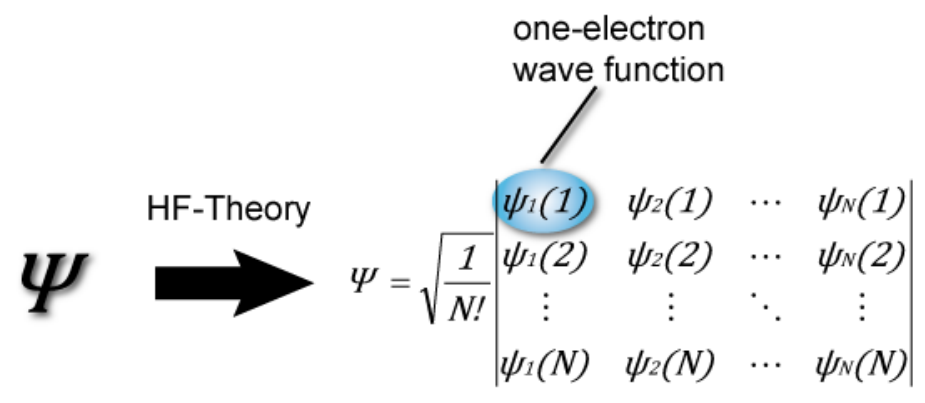

Figure 2-2. Representation of the wavefunction by a Slater determinant.

Every electron is described by its own spin orbital, which is delocalized over the whole molecule. How can we extract chemical meaningful information from $\Psi$, so that we can retrieve popular empirical chemical concepts from the quantum mechanical description? According to Lewis atoms in a molecule are held together by chemical bonds, which consist of two electrons of antiparallel spin, called electron pair. ${ }^{[68]}$ If such an electron pair belongs only to one nucleus, it is denoted a lone pair. Simply by demanding that electron pairs should avoid each other because of Pauli repulsion the Lewis theory leads to the valence shell repulsion theory (VSEPR), which successfully predicts the three dimensional structure of many molecules (figure 2-3).

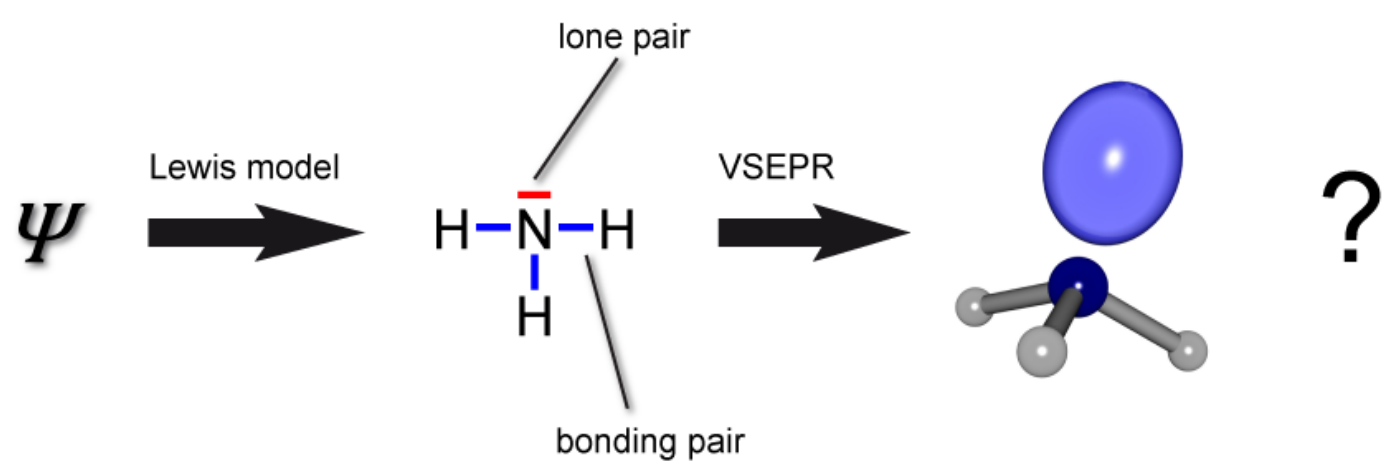

Figure 2-3. Is there a link between the wavefunction and chemical concepts?

It has been a major effort to bridge the gap between the wavefunction and these chemical models during the last decades. Among the earlier attempts valence bond (VB) theory, ${ }^{[69]}$ natural bond orbital (NBO) analysis, ${ }^{[70]}$ Hirshfeld partitioning ${ }^{[71]}$ and Mulliken population analysis $^{[72]}$ have to be mentioned. Nevertheless, in the following two relatively new approaches for this task are outlined. The first is Bader's Quantum Theory of Atoms in 
Molecules (QTAIM). ${ }^{[73]}$ Instead of analyzing the wavefunction, QTAIM focuses on the topological analysis of $\Psi^{2}$. The benefit is that contrary to $\Psi$, the product of this function with its complex conjugate has a physical meaning, which is the electron density distribution of the molecule. Since these can be obtained both by theory and experiment, QTAIM is in a predestinated position compared to other theories which are only applicable to the calculated abstract wavefunction.

The second method used is the Electron Localization Function (ELF) ${ }^{[74]}$ which was developed by Becke and Edgecombe, or rather its successor, the Electron Localization Index $(E L I)^{[75,76]}$ introduced by $M$. Kohout. Both ELF and ELI try to localize electron pair regions in molecules and concatenate them with the traditional Lewis picture. QTAIM and ELF/ELI provide a rigid definition of what a chemical bond is. This is important, since often experimental chemists already seem to know exactly which atoms in a molecule are connected. Thereby it is often overlooked that no clear definition of the chemical bond exists. In the nineteenth century the concept of the chemical bond has rather been developed on the basis of regularities in the proportion between the elements with respect to other elements than on quantum chemical grounds. During that time the term valency describing the maximum connectivity of an atom has been founded. For example in methane the carbon atom has a valency of four, but does this automatically imply that carbon is also bonded to these four hydrogen atoms? Furthermore, on which basis is the carbon atom considered to be bonded to hydrogen but no such interaction is postulated between the hydrogen atoms? Since chemist's association of the chemical bond is rather vague it is no surprise that empirical bonding theories fail to explain bonds in molecules like organolithiums or boranes, where it is not automatically "clear", which atoms are bonded and which are not. ${ }^{[7]}$ This issue clarifies the importance of theories like QTAIM or ELF/ELI, which put the concept of the chemical bond on a more solid (quantum chemical) basis.

In practice the coordinates obtained by an X-ray experiment served as the starting geometry for quantum chemical calculations. Then a gas phase optimization with Turbomole or Gaussian was performed and the resulting wavefunction was analyzed by the DGRID ${ }^{[78]}$ program from M. Kohout. DGRID calculates property values like the ED or ELI (and many others) on an equidistant grid (scheme 2-1). Later a topological analysis including a basin search can be performed numerically on the grid data. 


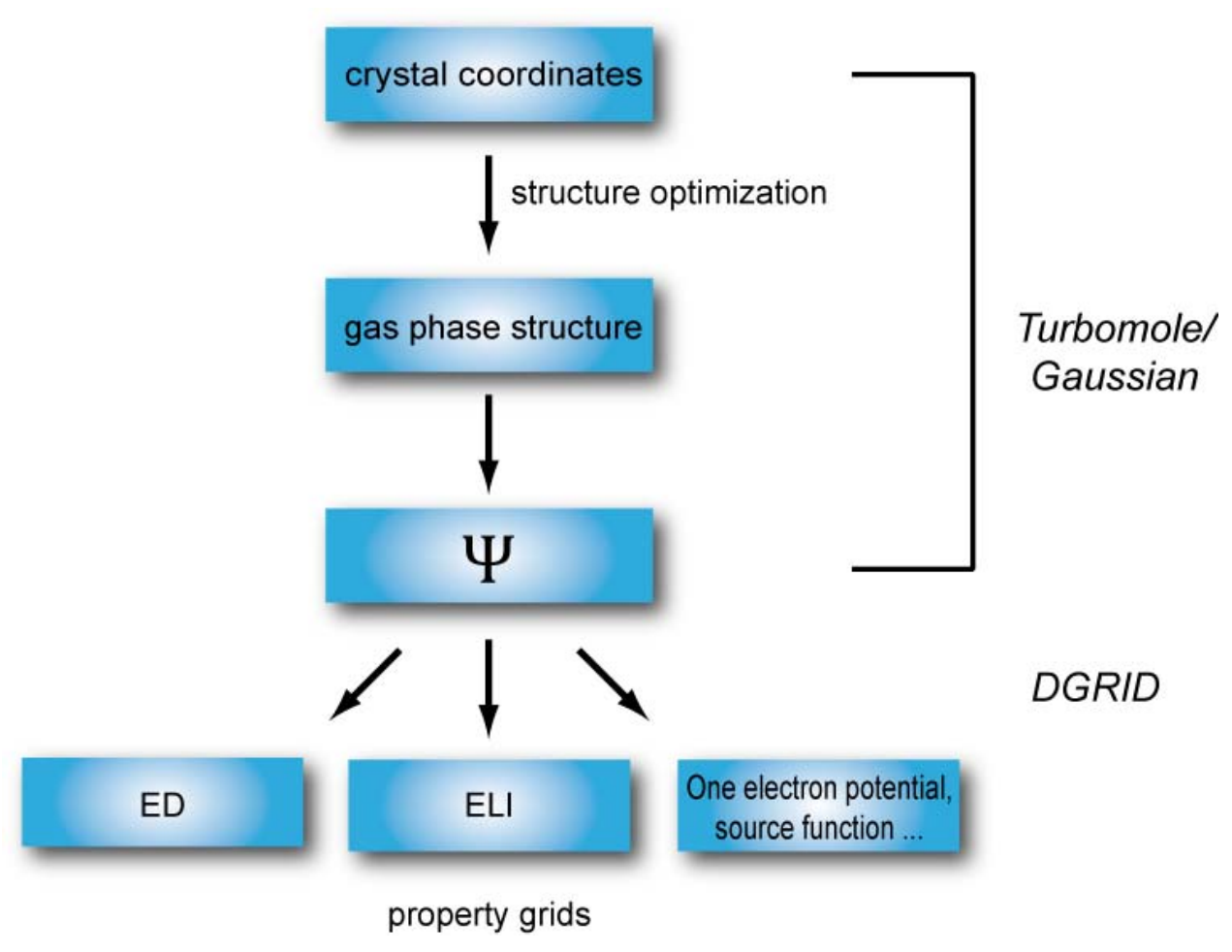

Scheme 2-1. Work flow for the quantum chemical calculations.

\subsubsection{QTAIM}

Bader's QTAIM ${ }^{[73]}$ is a real space partitioning scheme and performs a topological analysis of the electron density. The first question that arises when looking at the electron density is, how is it possible to segment it into its atomic contributions, or in the words of QTAIM, what is an "atom in a molecule"? It can be shown that the gradient vector field naturally partitions the electron density into atomic basins. These are encompassed by surfaces of "zero-flux", which mark the borders between two atoms (figure 2-4).

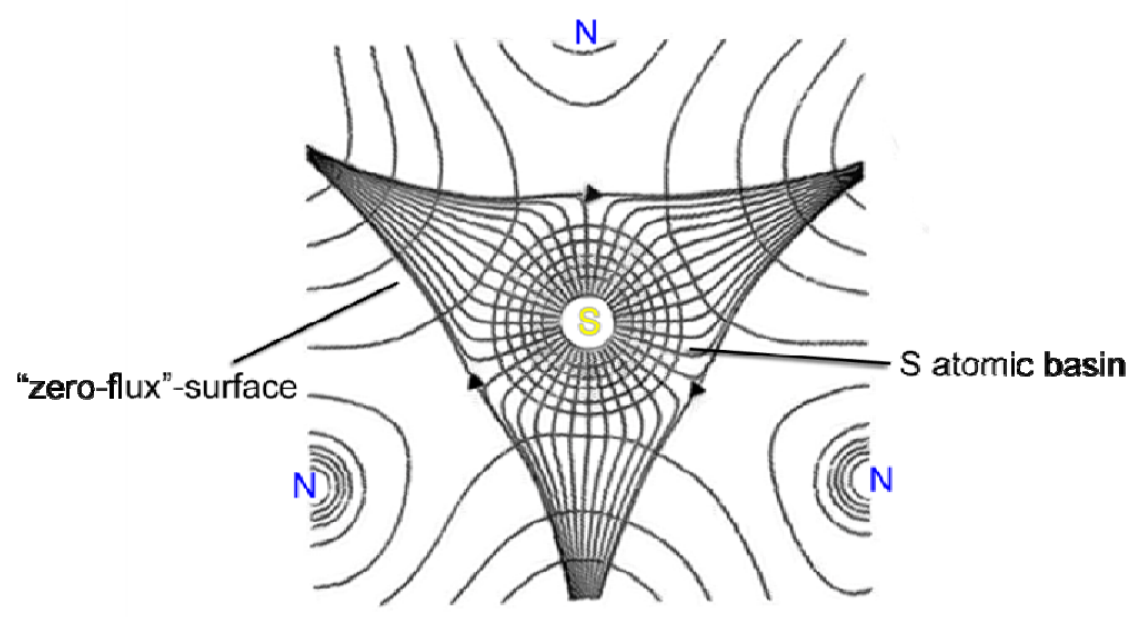

Figure 2-4. Gradient vector field of $\mathrm{S}\left(\mathrm{NH}_{3}\right)_{3}$. 
Mathematically, the zero-flux surfaces are defined as $\nabla \rho(\mathbf{r}) \cdot \mathbf{n}=0$, where $\mathbf{n}$ is the outward directed normal vector. They are also termed interatomic surfaces (IAS). All lines of the gradient vector field end up at the position of a nuclear attractor. Thus every atom consists of an attractor and its corresponding basin. Since all points within the volume of the atomic basin belong to one nucleus, integrating the density over the basin yields the AIM charge of the atom. Figure 2-5 depicts the atomic basin of the sulfur atom in $\mathrm{S}\left(\mathrm{NH}_{3}\right)_{3}$, which nicely shows the deformation of the spherical symmetry due to bonding to three nitrogen atoms.

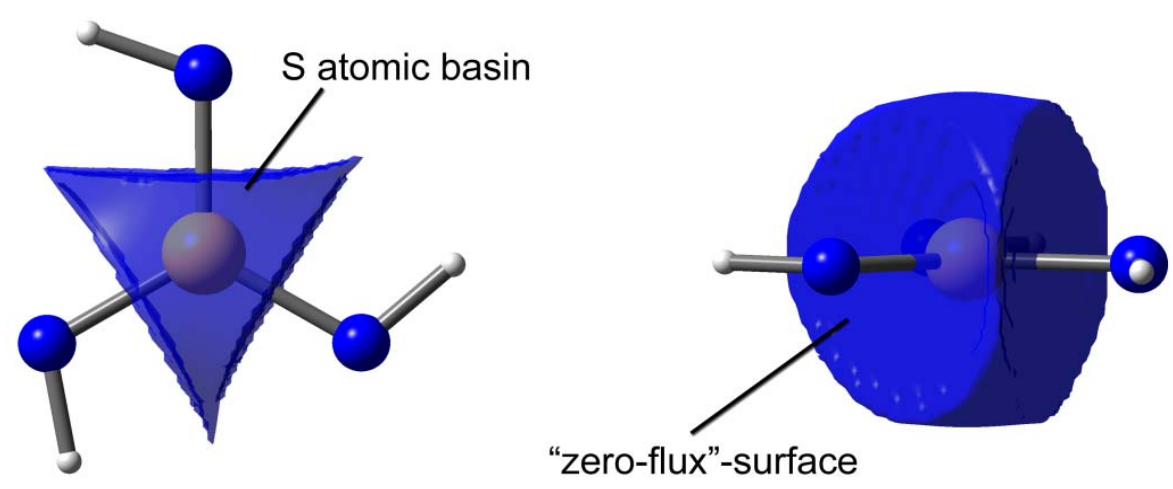

Figure 2-5. Sulfur atomic basins in $\mathrm{S}\left(\mathrm{NH}_{3}\right)_{3}$.

After the definition of an atom in a molecule it is necessary to find a criterion for a bond between two atoms. This information can be extracted from the electron density by a topological analysis. Since the electron density is a 3-dimensional function there are four types of extrema present: Local maxima, local minima and two types of saddle points. These extrema, also called critical points (CP), are classified by using the Hessian of the electron density. This $3 \times 3$ second derivative matrix yields three eigenvalues and three associated eigenvectors. Critical points are classified according to the number of nonzero eigenvalues and the signature, which is the sum of the sign of the curvatures at the CP. Accordingly, local maxima are denoted at $(3,-3)$ critical points. They usually correspond to nuclear positions, although non-nuclear attractors (NNA) exist in metals also. ${ }^{[9]}$ Saddle points in $\rho(\mathbf{r})$ are denoted as $(3,-1)$ critical points. Here two curvatures are negative and $\rho(\mathbf{r})$ is a maximum in the plane perpendicular to the atomic interaction line. In addition $(3,+3)$ and $(3,+1)$ CPs denote cage critical and ring critical points respectively. 


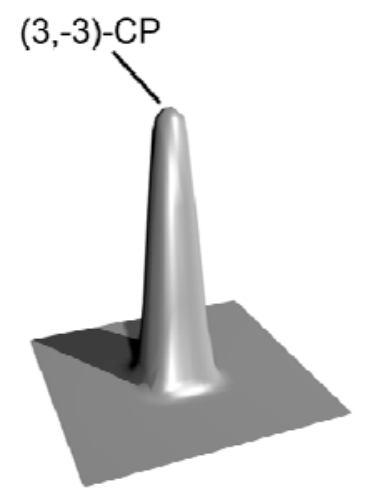

atomic position

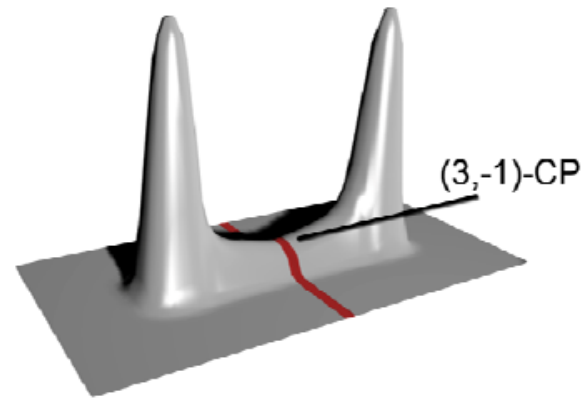

bond critical point

Figure 2-6. Classification of selected critical points in $\rho(\mathbf{r})$.

Within QTAIM $(3,-1)$ critical points are the first criterion for a chemical bond and thus are termed bond critical points (BCPs, figure 2-6). ${ }^{[80]}$ The electron density accumulation between two atoms alone is not sufficient to call them bonded though. The second criterion is that all forces on the nuclei must vanish. QTAIM is not applicable on non-eqilibrium geometries as they appear in molecular vibrations or transition states. If both criteria are fulfilled, two atoms are linked via a bond path. The bond path is defined as the line of maximum density between two atoms, reaching its minimum on the bond critical point. Eventually, the collection of all atoms and bond paths leads to the molecular graph, which can be interpreted as the most dominant Lewis structure. However, the meaning of an AIM bond is mainly an expression of attractive stability between two atoms and cannot always be related to a 2 center 2 electron bond. To examine the properties of the interaction and classify it in terms of chemical concepts like ionic, covalent, single/double bond, QTAIM introduces some additional analytic tools. The first is the analysis of the Laplacian, which is the second derivative of $\rho(\mathbf{r})$. The wealth of information contained in this function can be seen when comparing it to the electron density (figure 2-7). The ED possesses sharp maxima at the nuclear positions from which the density falls up rapidly. Structural features like lone pairs or valence shells cannot be visualized in $\rho(\mathbf{r})$ itself. However, the Laplacian of $\rho(\mathbf{r})$ reveals a fine structure with topological features, the so called VSCCs (Valence Shell Charge Concentrations), which might be interpreted as lone pair densities. 

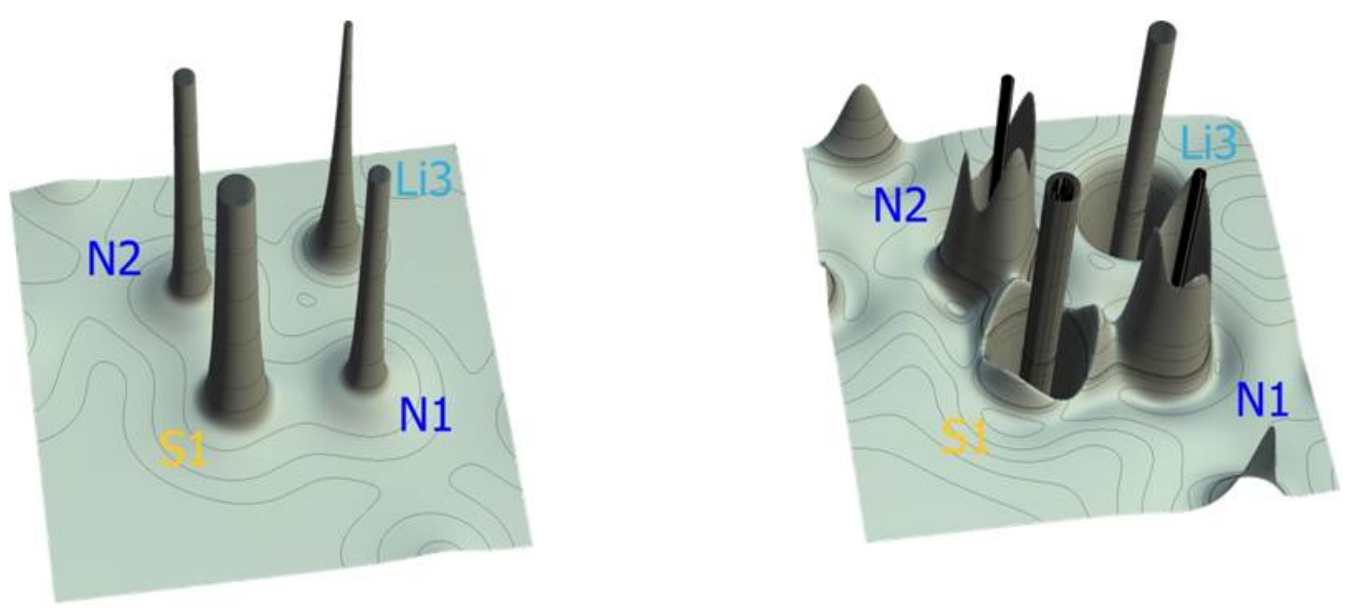

Figure 2-7. Differences between electron density $\rho(\mathbf{r})$ (left) and negative Laplacian $-\nabla^{2} \rho(\mathbf{r})$ (right), exemplary shown for the S1-N1-N2 plane of $\left[(\operatorname{thf}) \mathrm{Li}_{2}\left\{\mathrm{H}_{2} \mathrm{CS}(\mathrm{N} t \mathrm{Bu})_{2}\right\}\right]_{2} \cdot{ }^{[81]}$

Hence the second derivative of $\rho(\mathbf{r})$ acts as a magnifying glass on the electron density and reveals slight deformations which are induced due to the formation of chemical bonds. Bader summarized this behavior with the following words: "Just as the energy changes of interest in chemistry are but small fractions of the total energy of the system, so the changes induced to $\rho$ are only small 'ripples' in the total density."

The meaning of the Laplacian is the local curvature of the electron density at a given point. Since $\rho(\mathbf{r})$ is a three dimensional function, $\nabla^{2} \rho(\mathbf{r})$ is the sum of the three eigenvalues $\lambda_{1}, \lambda_{2}$ and $\lambda_{3}$. Hereby $\lambda_{3}$ displays the curvature along the atomic interaction line (AIL) and is always positive. On the contrary the two other eigenvalues $\lambda_{1}$ and $\lambda_{2}$ are always negative at the BCP and a measure of the curvature perpendicular to the bond path (figure 2-8). While $\lambda_{3}$ moves electron density away from the BCP, $\lambda_{1}$ and $\lambda_{2}$ are responsible for an accumulation of density through the IAS. The sign of $\nabla^{2} \rho(\mathbf{r})$ determines, which of the opposite curvatures dominates.

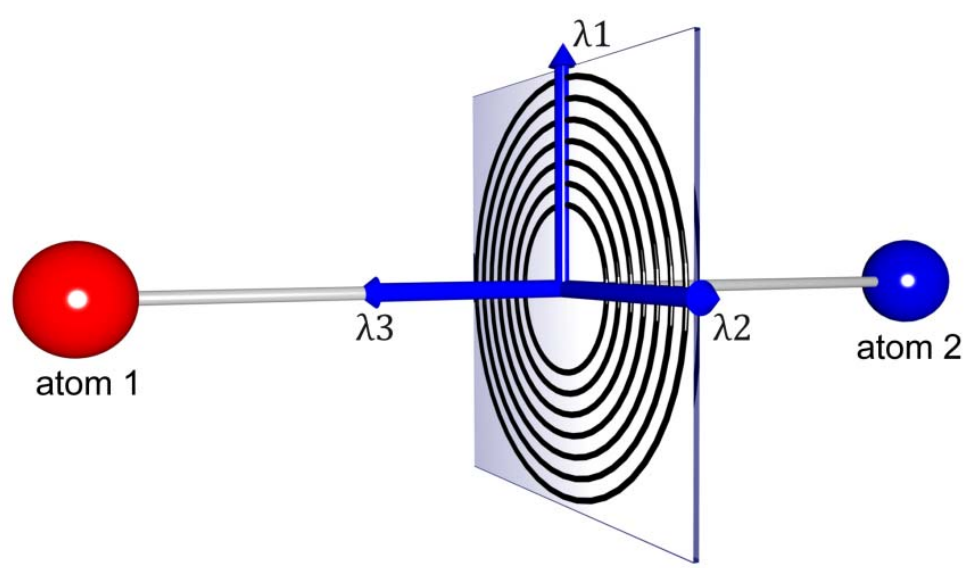

Figure 2-8. Orientation of the three eigenvalues of the Hessian matrix. 
The Laplacian helps to specify the character of the bond. ${ }^{[82]} \mathrm{A}$ negative sign for it shows that electron density is concentrated along the bond path. This is typical for a shared interaction how it takes place in covalent bonds. An example is the diatomic $\mathrm{N}_{2}$ molecule (figure 2-9, left). There are contour lines that encompass the whole molecule and the BCP is located symmetrically between both nitrogen atoms. The other extreme is a positive sign for the Laplacian. This occurs in the case of ionic bonds. There is no electron accumulation between the atoms but rather an electron transfer from the atomic basin of the electropositive element to the atomic basin of the electronegative atom. The attraction between the atoms is mainly electrostatic. This type of interaction is named closed shell interaction and exemplified with lithium hydride (figure2-9, right). Both lithium and hydride are isolated units, the hydridic anion being polarized in the direction to the lithium cation. The BCP is located in a region of charge depletion. An intermediate case can be found in polar bonds like they exist in carbon monoxide. It can be seen that electron density is shifted from the carbon to the oxygen atom and the BCP is settled near a rampant edge, a region where the $\nabla^{2} \rho(\mathbf{r})$ changes sign (figure 2-9, middle).
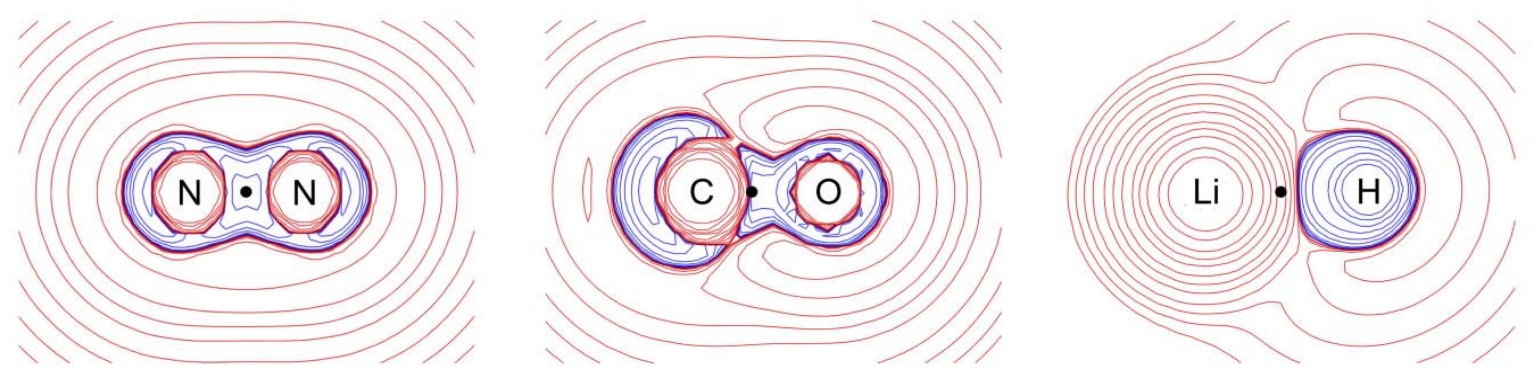

Figure 2-9. Laplacian contour maps of $\mathrm{N}_{2}, \mathrm{CO}$ and $\mathrm{LiH}$. Negative values are depicted by blue lines and positive values by red lines. The black dots assign the BCPs.

In summary, at the BCP ionic bonds have positive values for $\nabla^{2} \rho(\mathbf{r})$ and in addition a low value for $\rho(\mathbf{r})$. Typical covalent bonds are characterized by a negative value for the Laplacian in combination with a high value for $\rho(\mathbf{r})$, which is roughly an order higher than that for ionic bonds. ${ }^{[77]}$

A further application of the Laplacian is the identification of Lewis acidic and Lewis basic regions in a molecule. Molecules with a local non-bonding charge concentration behave as nucleophiles, while the ones with local charge depletion react as electrophiles. The local virial theorem associates acidic regions with zones where the electrons move faster on average. It is important to mention that the local charge concentrations predicted by the Laplacian coincidences in many cases with the corresponding properties of the highest occupied molecular orbital (HOMO). ${ }^{[83]}$

The third tool QTAIM offers is the ellipticity $\varepsilon$. It is defined as $\left(\lambda_{1} / \lambda_{2}\right)-1$ and gives a measure of the extent to which $\rho$ is elongated in one direction compared to another, both 
perpendicular to the AIL. $\varepsilon$ can be regarded as a quantitative index of the $\pi$-character of a bond.

The properties of a bond have been discussed so far only based on values at the BCP. However the concentration on a single point to characterize the whole bond is sometimes not sufficient, especially in the case of difficult bonding modes or if the BCP is located in a zone where $\nabla^{2} \rho(\mathbf{r})$ changes considerably. In this case a detailed investigation of the whole bond path gives additional and more reliable information. ${ }^{[84]}$

\subsubsection{ELI}

A different approach to bridge the wavefunction and chemical models is the Electron Localization Index $(\mathrm{ELI}) .{ }^{[75,76]} \mathrm{ELI}$ is advancement by $M$. Kohout to the electron localization function (ELF) that was introduced by Becke and Edgecombe. ${ }^{[74]}$ The ELF/ELI does not analyze the electron density, but the wavefunction itself. Since $\Psi$ is approximated by molecular orbitals (MOs), electrons are delocalized over the whole molecule, so chemical bonds cannot be visualized easily. The density accumulation encountered between a pair of atoms is the result of many small contributions of all MOs. Therefore, tools that restore "chemical bonds" between atoms which clearly show the connectivity of elements in a molecule are needed to obtain classical Lewis formula. This can be achieved by producing a set of localized molecular orbitals (LMOs). ${ }^{[62]}$ These have the characteristics that they are spatially confined to a small volume and have virtually no contributions outside this volume. It is important to note that this transformation is purely mathematical since the total wavefunction remains unchanged. The only thing that changes is the choice of orbitals, which linear combinations form $\Psi$. However this transformation is somehow arbitrary. This leads to the fact, that different localization schemes can yield different descriptions of the same molecule. An example is the bonding type in ethylene. In the Pikek-Mezey localization scheme the double bond consists of a $\sigma$ and a $\pi$ bond. On the contrary, the Boys procedure leads to two equivalent banana bonds. Since both sets of LMOs produce the same total wavefunction and hence the same electron density, from this point of view these different descriptions of the double bond can't be distinguished. This ambiguity led to the development of ELF, which is an orbital-independent measure of electron localization. The idea behind ELF is simple: In the Lewis picture bonds are composed of electron pairs being shared equally between two atoms. Consequently, Edgecombe et al. searched for a way to localize electron pairs in a molecule, hence finding a mathematical function describing the electron pair density. Their procedure can be explained ostensive by imaging an electron pair that is located in the center $\mathbf{r}$ of a hypothetical sphere with radius $s$ (figure 2-10). The ELF calculates now the probability of a second electron pair within the sphere. This pair density 
can be approximated by the probability of two same spin electrons inside the sphere because every electron pair consists of two electrons with opposite spin. If the probability of finding a same spin electron close to the reference electron is high, the electron pair localization at this point in space is low. On the contrary, if the probability to find a same spin electron nearby is low, the electron pair localization is high.

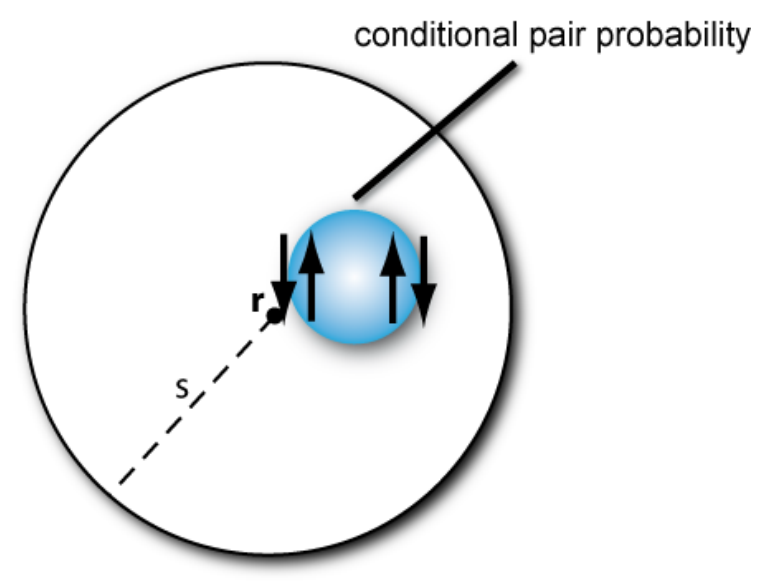

Figure 2-10. Probability to find a same spin electron close to a reference electron at reference point $\mathbf{r}$ within a shell of radius $\mathrm{s}$.

Mathematically the probability of finding two electrons of same spin $\sigma$ simultaniously at position 1 and $\mathbf{2}$ is given as

$$
P_{\Omega}^{\sigma \sigma}(1,2)=\rho_{\sigma}(1) \rho_{\sigma}(2)-1 \rho_{\sigma}(1,2)^{2} \quad \text { Eq.2-2 }
$$

Next we ask for the probability of finding a second same spin electron near a reference electron, which is located at $r$ with certainty. This is done by divding Eq. $2-2$ by $\rho_{\sigma}(\mathbf{1})$ and the conditional pair probability is obtained.

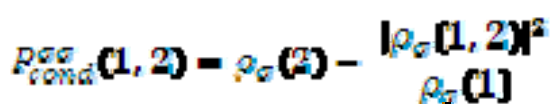

Since we are only interested in the probability of finding a same spin electron close to another, e.g. in the above mentioned sphere, and not elsewhere in the molecule, it is possible to replace Eq. 2-3 by a Taylor expansion of the spherical averaged conditional pair density for small $s$ and with $\tau_{0}$ being the kinetic energy density.

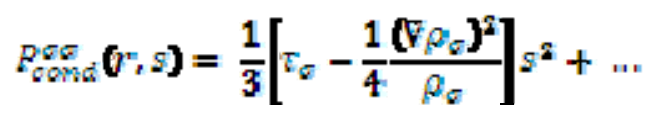

In the Taylor series, the first s-indepent term vanishes because of the Pauli principle and the term that linearly depends on $s$ vanishes because of Kato's cusp condition. ${ }^{[85]}$ The 
quadratic expression in Eq. 2-4 is thus the leading term and the coefficient of $s^{2}$ can be interprated as measure of electron localization.

$$
D_{\sigma}=\tau_{\sigma}-\frac{1}{4} \frac{\left(\bar{\varphi} \rho_{\sigma}\right)^{2}}{\rho_{\sigma}}
$$

It is a little bit distracting, that high values for $D_{\sigma}$ are indicators for a low localization and low values for a high localisation vice versa. Therefore, the final ELF has been defined as

$$
E L F=\left(1+x^{2}\right)^{-1}
$$

with ${ }^{2 \approx}=\frac{D_{\varepsilon}}{D g}$ and $D !$ corresponding to the ELF in the homogeneous electron gas. The transformation in Eq. 2-6 also ensures that ELF is restricted to values between zero and one. A value of one denotes perfect localization, while a value of 0.5 represents perfect delocalization.

While this transformation yields very convenient results, there is some criticism on the introduction of the arbitrary reference of the homogeneous electron gas. Indeed, this

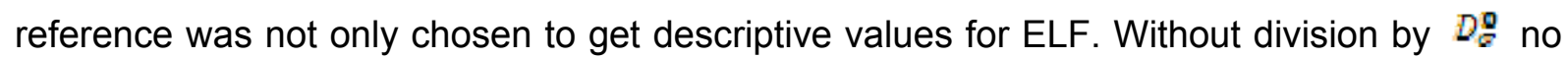
meaningful chemical structure can be recovered from the same spin pair probability. While in ELF this arbitrary chosen reference is essential, the new developed electron localization index (ELI) of M. Kohout does not suffer from this dependency. ELI partitions the whole space into a finite number of small space regions $\Omega_{q}$ which encompass a fixed charge. The integral of the same spin electron pair density within each region tells how many same spin electrons contribute to the charge of $\Omega_{q}$. Low values mean that most of the charge originates from a single $\sigma$-spin electron, which is equivalent to a high degree of localization. Higher values reflect the participation of many same-spin electrons to the fixed charge in $\Omega_{q}$ and the localization decreases. So in contrast to ELF the final ELI is not bound to values between zero and one.

The analytical procedure to interpret the ELF/ELI exhibits some similarities to QTAIM. At the beginning the maxima in the ELI are determined. These maxima are termed attractors. Subsequently the space can be partitioned by assigning each point to an attractor by following the path of highest ascent, which is the gradient vector field of ELI. In the case of closed-shell systems, all points in space, which belong to one attractor, represent the localization domain of an electron pair. Thus while QTAIM segments the molecule only in atomic basins, the ELI performs a more precise partitioning into electron pair localization basins. These can be divided into different classes. The synapticity of a basin states how many atoms the basin is connected to. Monosynaptic basins belong only to one nucleus. They consist of core basins or nonbonding electrons pairs. As an example, ELI is able to 
recover the whole shell-structure of the krypton atom. Four separated localization shells and their contraction can be observed (figure 2-11. left). ${ }^{[86]}$ For chemical bonding analysis disynaptic basins are interesting. They are shared between two nuclei and correspond generally to a classical 2 electron 2 center bond. Likewise double bonds can be visualized. In ethene two disynaptic ELI basins can be found along the carbon-carbon trajectory, which are located above and below the molecular plane (figure 2-11, right).
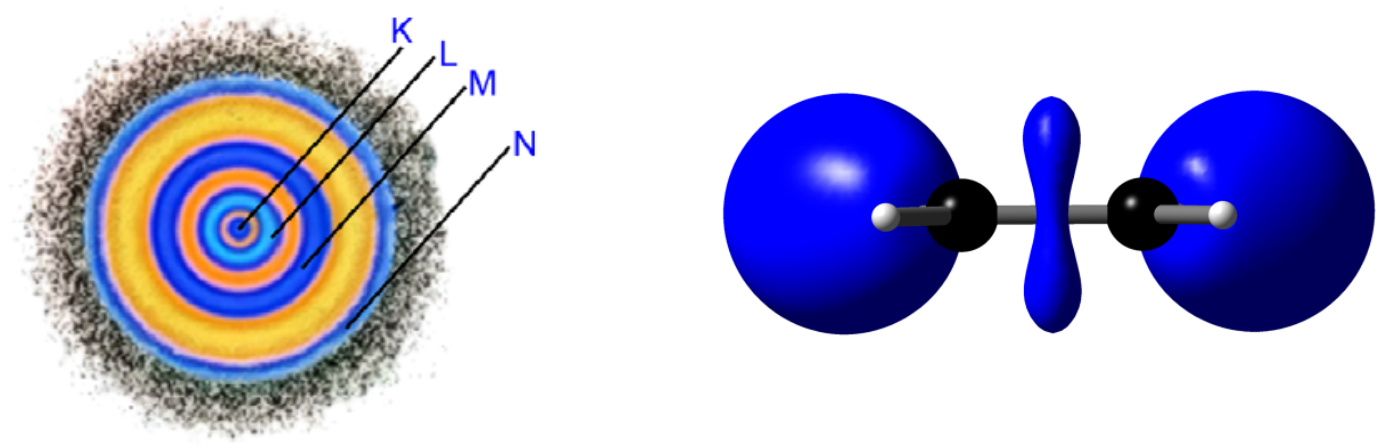

Figure 2-11. ELI contour plot displaying the shell structure of the krypton atom (left) and ELI isosurface representation of the double bond in ethene (right). Blue colors indicate high ELI values.

Furthermore, the ELI is able to relate the position of an attractor to the degree of polarity in a chemical bond. ${ }^{[86]}$ In the case of perfect covalent bonding the attractor is located exactly in the middle of the bonded atoms. If the polarity increases, the ELI maximum migrates towards the more electronegative element.

The pictures obtained by ELI show similar features like the QTAIM results. Both tools are able to deduce the information needed for an understanding of chemical reactivity from quantum mechanics. Combined they can be used for the verification of new interesting molecular interactions, which exceed the scope of existing empirical models. 


\section{ORganoliththiUm REAgents}

\subsection{2-AnisolLi·pmdeta}

\subsubsection{Introduction}

Many natural products that are of interest for the pharmaceutical and agrochemical industry contain substituted aromatic or heteroaromatic rings. The regiospecific functionalisation of these aromatic building blocks is a major research area in organic chemistry. Among the techniques that are employed to introduce substituents into aromatic rings are the electrophilic aromatic substitution (Friedel-Crafts) and its counterpart the nucleophilic aromatic substitution. ${ }^{[3]}$ In addition to these classical organic reaction types exists a powerful methodology using organometallic reagents to functionalize aromatics. The Directed ortho Metalation (DoM) is one of the most used approaches to transform singly substituted aromatics into more complex ones. ${ }^{[23]}$ The first example for this type of reaction, the ortho deprotonation of anisole, was discovered independently in 1939/1940 by Gilman and $B e b b^{[87]}$ as well as Wittig and Fuhrmann. ${ }^{[88]}$ In the DoM reaction, the aromatic compound is being metalated in ortho position to a functional group with a high regioselectivity. Precondition for the ortho deprotonation is that the functional group exhibits some directing and/or acidifying effect of an adjacent hydrogen atom. Even so the DoM process requires a very strong deprotonation reagent. Traditionally, this is the domain of alkyllithium bases. To further enhance their reactivity (by deaggregation) these are usually employed in polar solvents like ether, thf or in the presence of the diamine ligand tmeda.

Besides the undoubted importance and usefulness of this reaction, there is still a continuing debate on the mechanism and the origin of the regioselectivity. The reactivity of the lithiation of benzene and anisole differ by the factor $1000 .{ }^{[89]}$ Early it has been proposed that inductive effects acidify the ortho protons in anisole, explaining the higher reactivity as well as the ortho selectivity. ${ }^{[90]}$ Newer models include the concept of a precomlexation step with a discrete lithium-substituent interaction. Scheme 3-1 shows a reaction pathway involving the complex induced proximity effect (CIPE) as proposed by Beak et al. for the deprotonation of anisole. ${ }^{[22,91]}$ In the first step the organolithium reagent is coordinated to a Lewis basic heteroatom of the directing metalation group (DMG), bringing the carbanionic $\alpha-$ carbon in close proximity to one hydrogen next to the DMG. In the next step the ortho hydrogen is abstracted resulting in a lithiated intermediate, which is considered to be stabilized by an oxygen lithium interaction. Subsequent quenching with an electrophile leads to ortho substituted anisole. 


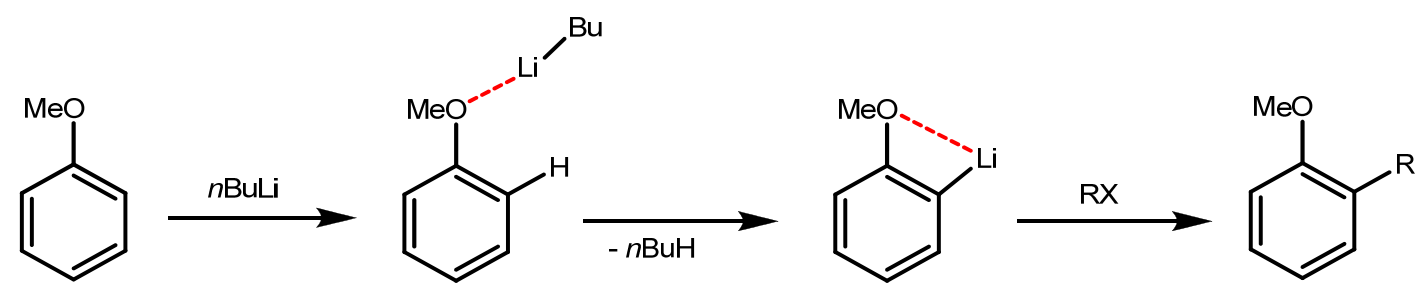

Scheme 3-1. CIPE model for the DoM of anisole. ${ }^{[22]}$

Collum et al. ${ }^{[89]}$ published a different mechanism depicting the ortho selectivity and posed the provocative question: "Are $n B u L i / t m e d a$ mediated arene ortholithiations ortho-directed?" On the basis of rate studies they suggest a mechanism via dissolvated dimers and triple ions (scheme 3-2), in which the occurrence of a CIPE precomplex is not a necessity. Indeed, spectroscopic results approve that $n \mathrm{BuLi}$.tmeda is dimeric in hydrocarbons. ${ }^{[92]}$ In accordance, their kinetic results of the ortho lithiation suggest a stochiometry of the transition state of $\left[(n \mathrm{BuLi})_{2}(\text { tmeda })_{2}\right.$ (anisole $\left.)\right]$.

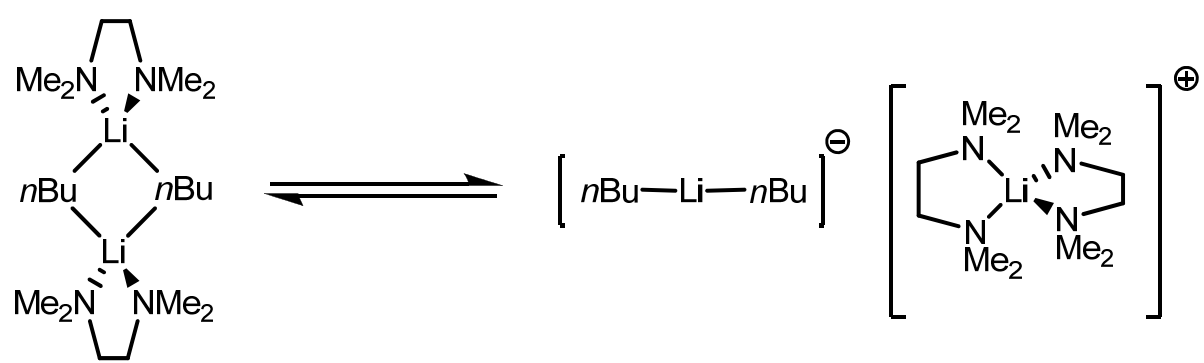

Scheme 3-2. Formation of $n$ BuLi triple ions.

Additional computational studies support a mechanism via the participation of triple ions. Triple ions strongly resemble the qualities of heterobimetallic ate complexes and should have an increased basicity because of the additional negative charge. Scheme 3-3 outlines the whole mechanism. The reason for the ortho selectivity is mainly founded in inductive effects. However, Collum emphasizes explicitly that these results are only valid for the DoM of anisole and do not contradict Beak's CIPE theory in general. In the presence of stronger DMGs a pre-metalation complex could still be possible. 


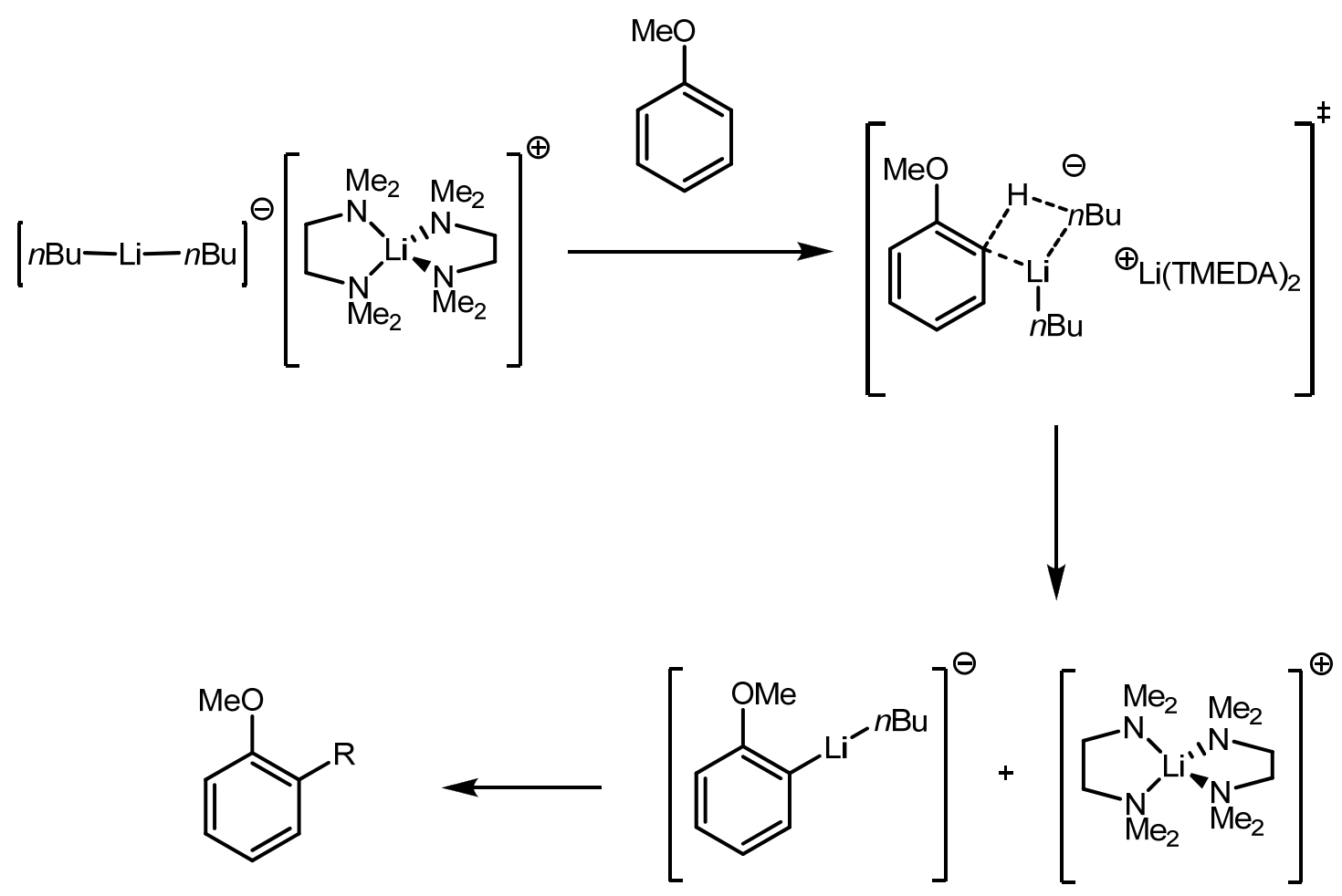

Scheme 3-3. Ortho lithiation of anisole via triple ions.

While NMR and kinetic studies give an impression on the reaction in solution, X-ray structure determination in the solid state can yield valuable information on the exact geometry of the involved species. Often, solid state structures of solvated molecules are retained in solution thus giving important information on the reaction pathway. For example, the already mentioned $n \mathrm{BuLi} /$ tmeda is dimeric in apolar solvents as in the solid state. Harder et al. were able to elucidate the structures of lithiated anisole compounds. They obtained donor free 1-Lithio-2-methoxybenzene in crystalline form by a lithium bromine exchange with $n$-butyllithium and 1-bromo-2-methoxybenzene in pentane. The solid state structure showed that 1-Lithio-2-methoxybenzene aggregates to a tetramer, consisting of a $\mathrm{Li}_{4}$ tetrahedron which faces are capped by carbon atoms. (figure 3-1). ${ }^{[93]}$ 


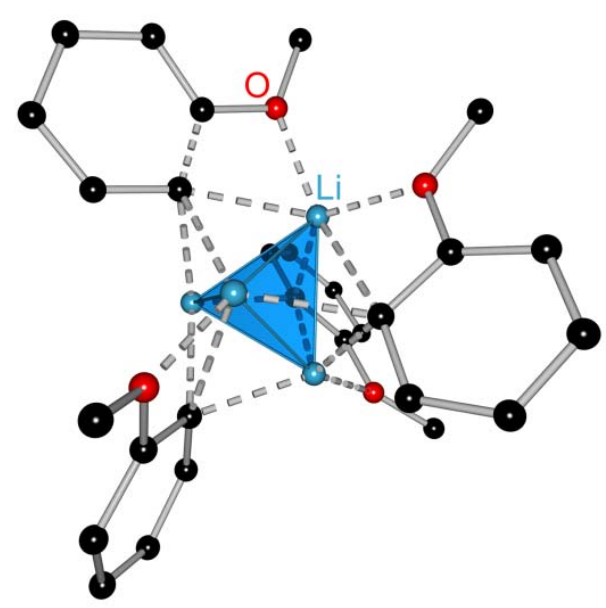

Figure 3-1. X-Ray structure of donor free 1-lithio-2-methoxybenzene. ${ }^{[93]}$

They also deprotonated anisole with $n \mathrm{BuLi}$ in hexane with two equivalents of tmeda. Addition of this bidentate amine ligand normally breaks up lithium clusters resulting in smaller aggregates. Interestingly, in this case they found that in the solid state tmeda bridges two symmetrically equivalent lithium tetrahedrons thus leading to the even bigger aggregate [1-

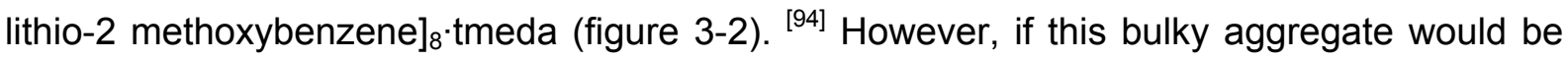
retained in solution, it would contradict kinetic data which show high reaction rates. Moreover, this molecular structure is not concordant with the solvent separated $[\mathrm{Ar}-\mathrm{Li}-n \mathrm{Bu}]^{-}$ $\left[(\text { tmeda })_{2} \mathrm{Li}\right]^{+}$proposed by Collum for a mechanism via triple ions.

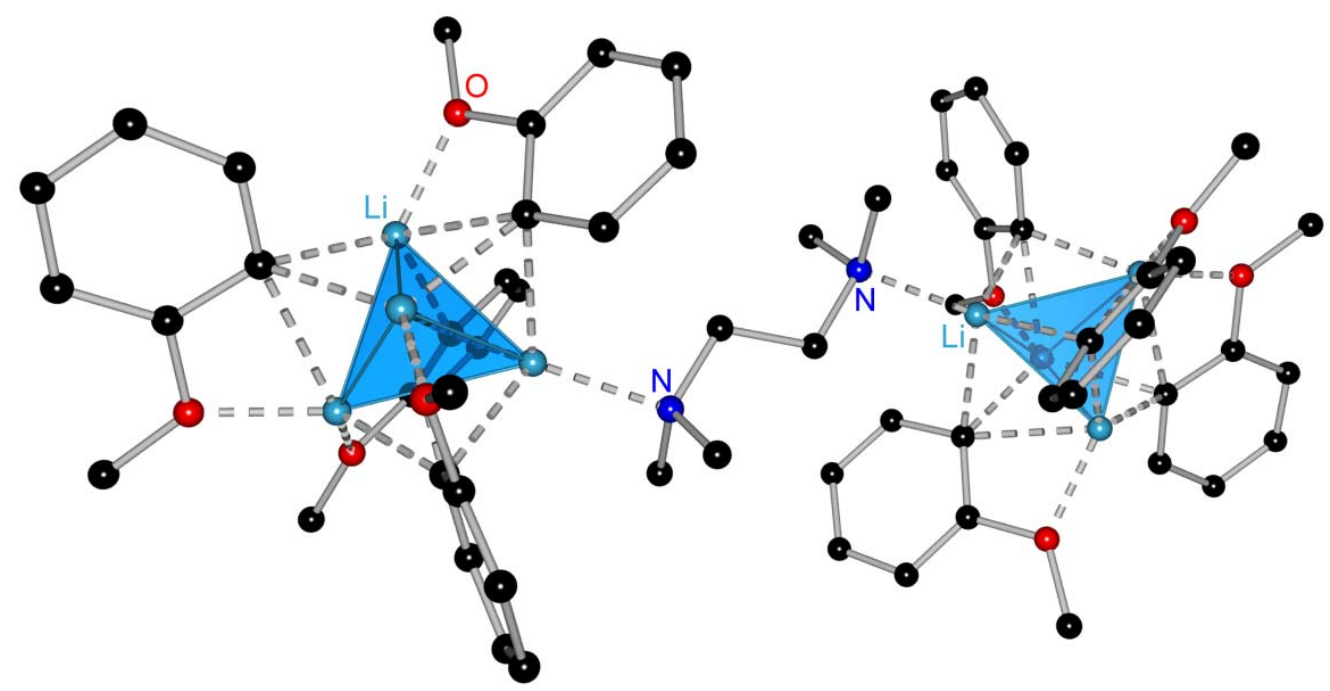

Figure 3-2. X-Ray structure of [1-lithio-2 methoxybenzene] $]_{8} \cdot$ tmeda $^{[94]}$

A similar coordination behavior where tmeda fails to reduce aggregation can be found in the crystal structure of $\left[(\text { tmeda })_{2}(\mathrm{MeLi})_{4}\right]_{\infty .}{ }^{[95]}$ Instead of chelating one lithium atom with both 
nitrogen atoms, tmeda links $(\mathrm{MeLi})_{4}$ tetramers and produces a three dimensional network, leading to an insoluble polymer (figure 3-3).

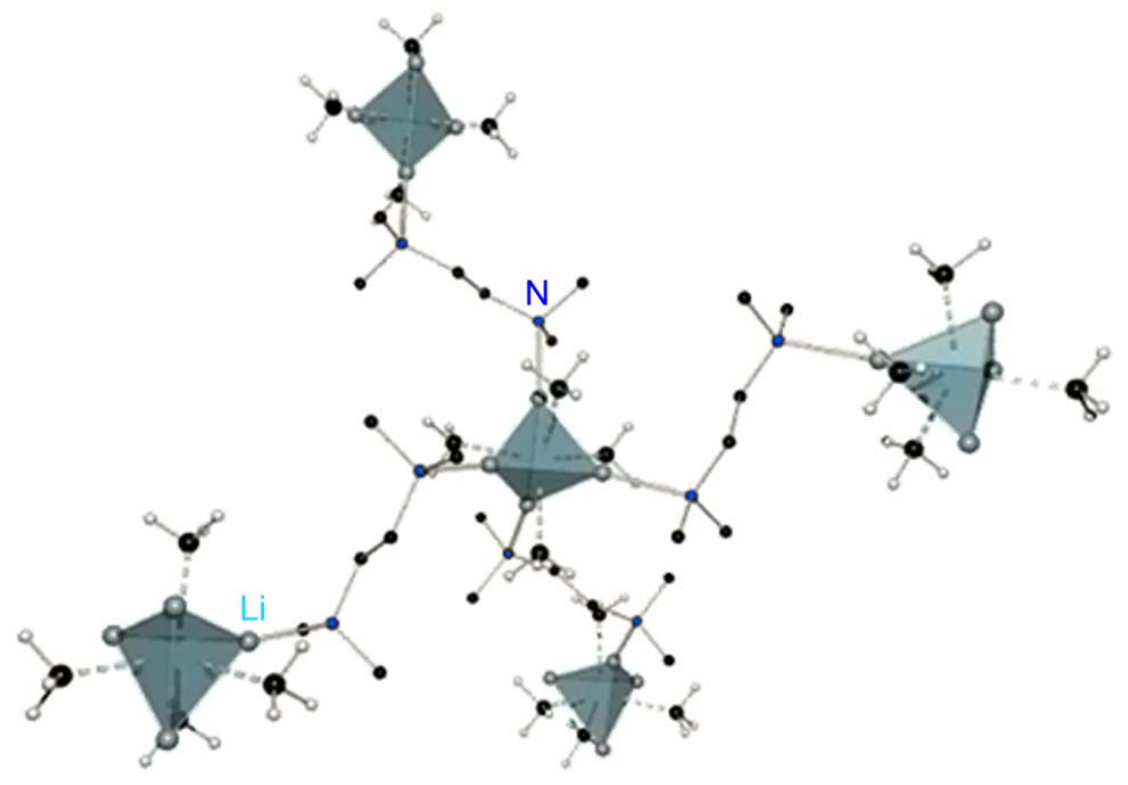

Figure 3-3. Solid state structure of $\left[(\mathrm{MeLi})_{4}(\mathrm{tmeda})_{2}\right]_{\infty}{ }^{[95]}$

To compare the solid state structures with solution structures Harder et al. performed NMR-experiments on ortho lithiated anisole in donor free solvents and in the presence of thf, tmeda and pmdeta. ${ }^{[93]}$ For solution studies NMR spectroscopy can yield important information on the structure and aggregation state of organolithiums, especially when ${ }^{6} \mathrm{Li}$ enriched samples are available. Here the situation seems to be partly different. The solid state structure of 1-Lithio-2-methoxybenzene is retained in solution. The low temperature ${ }^{13} \mathrm{C}$ NMR of donor free 1-Lithio-2-methoxybenzene in toluene- $d_{8}$ shows, that the ortho carbon signal is splitted into seven lines, indicating that it is bond to three ${ }^{6} \mathrm{Li}$ nuclei. This is furthermore supported by the ${ }^{13} \mathrm{C}-{ }^{6} \mathrm{Li}$ coupling constant of $5.1 \mathrm{~Hz}$, which is typical for a carbon capping a lithium triangle. In contrast, when two equivalents of tmeda are added to the solution instead of a septet the signal of the lithiated carbon becomes a quintet and the ${ }^{13} \mathrm{C}-{ }^{6} \mathrm{Li}$ coupling constant raised to $6.8 \mathrm{~Hz}$, suggesting only two carbon lithium contacts. Apparently, in solution tmeda is able to break the tetramer down to a dimer and the solid state structure reveals just the aggregate with the lowest solubility.

Since organolithium chemists are often interested in producing monomers, Harder et al. employed the tridentate donor ligand pmdeta to achieve further deaggregation. Indeed, their NMR studies showed that the resonance of the lithiated carbon is now split into a triplet $\left(\left(J\left({ }^{13} \mathrm{C}-{ }^{6} \mathrm{Li}\right)=13.6 \mathrm{~Hz}\right)\right.$, which is indicative of one carbon-lithium bond. They conclude that 2AnisolLi pmdeta is monomeric in solution and state that it "seems likely that that the ligand is coordinated to lithium with all its three nitrogen donor atoms,...; because of the rigidity of the 
methoxybenzene anion, at least weak intramolecular coordination of the methoxy substituent must be assumed in this monomer." ${ }^{[93]}$ However, presumably due to the high reactivity no crystal structure of 2-AnisolLi-pmdeta or another monomeric lithiated anisole compound can be found in the CCDC. ${ }^{[96]}$ Therefore the crystallization and X-ray analysis of this very sensitive monomer was achieved to get a definite picture of this molecule and the complexation of the lithium cation. Based on the atomic coordinates of an X-ray analysis computational studies can then be performed to get a deeper insight into the bonding situation.

\subsubsection{Preparation}

2-AnisolLi-pmdeta (1) was prepared by the addition of $n$-butyllithium and the tridentate donorbase pmdeta to a solution of anisole in hexane (scheme 3-4). The resulting solution was refluxed for 2 hours and stored at $-24{ }^{\circ} \mathrm{C}$ over night. The next day air and moisture sensitive crystals were obtained, that already had a light brown color due to decomposition. These were suitable for an X-ray structure analysis and 1 was obtained as a monomeric aggregate in which lithium was co-coordinated by pmdeta.

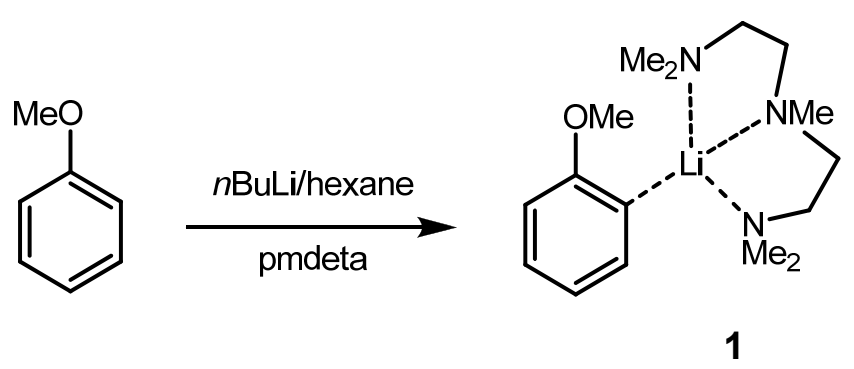

Scheme 3-4. Ortho lithiation of anisole.

The compound crystallizes in the monoclinic space group $P 2_{1} / n$. The lithium cation is tertracordinated by the deprotonated carbon at $\mathrm{C} 1$ and three nitrogen atoms of the donor base pmdeta (figure 3-4). 


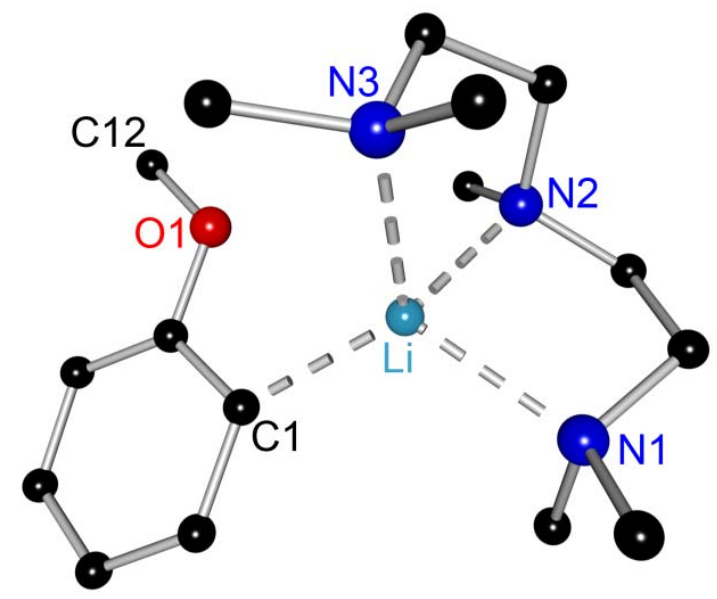

Figure 3-4. Molecular structure of monomeric 2-AnisolLi-pmdeta (1). Hydrogen atoms are omitted for clarity.

The C1-Li1 bond length of 210.9(3) pm is remarkably shorter than in the solvent free tetrameric 1-lithio-2-methoxybenzene $(229.5 \mathrm{pm}) \cdot{ }^{\left[{ }^{[93]}\right.}$ This reflects the trend that carbon-lithium distances in monomers usually tend to be shorter than in tetramers since the charge is concentrated only between one lithium and one carbon atom. ${ }^{[10]}$ The $\mathrm{N}-\mathrm{Li}$ donor bonds are in the range of 214-215 pm and are slightly longer than the primary C1-Li1 contact. The question arises whether the coordination sphere of lithium is completed by now. Although a tetrahedral coordination of lithium is found in many examples, this does not originate from the fact that lithium wants to attain a Lewis electron octet. Rather lithium-anion interactions are electrostatic in nature so the metal center will be surrounded by as many ligands sterically possible. $\mathrm{O} 1$ possesses two nonbonding electron pairs, which could provide electron density towards the electron deficient lithium cation, since the backside of Li1 is not shielded completely by the pmdeta ligand. That is because this half-space is sterically occupied by only one $\mathrm{N}$-methy group, while the opposite side is shielded by two $\mathrm{NMe}_{2}$ groups. Moreover, the bond angles between $\mathrm{C} 1$, Li1 and the $\mathrm{NMe}_{2}$-nitrogens forward-tuned C1-Li1-N1 $\left(117.37(14)^{\circ}\right.$ and C1-Li1-N3 $\left(114.90(14)^{\circ}\right)$ are considerably smaller than the C1-Li1-N2 angle $\left(133.11(15)^{\circ}\right)$ to the NMe group pointing backwards. Thus for steric reasons an oxygen-lithium donor bond would be possible. However, although the orientation of the oxygen lone pairs cannot be deduced from a standard X-ray experiment, it can be asserted that the O1-Li1 distance of 296.8(3) pm is notably longer than the $\mathrm{N}-\mathrm{Li}$ donor bonds. Therefore, already at this point a stabilization effect of 1 through an interaction between the methoxy group and lithium is questionable. The only effect that the methoxy group induces in the structure is a tilt of pmdeta so that the angle between the anisole ring and the plane containing Li-N2-C12 is $40.4^{\circ}$. This tilt can be accredited to a minimizing of steric repulsion between anisole's methoxy group and the methyl group at N2 of pmdeta. In 
solvent free tetrameric 1-lithio-2-methoxybenzene the situation is more ambiguous. On the one hand there are short $\mathrm{Li}-\mathrm{O}$ contacts present $(195.5 \mathrm{pm})$. On the other hand these interactions are only present for three of the four lithium atoms, while the coordination sphere of Li4 shows no such contacts. Harder et al. ascribe the absence of a Li4-O contact to a long range van der Waals interaction (291 pm) between Li4 and one phenyl ring carbon of an adjacent tetramer. However, in total it remains unclear whether short atomic distances in a crystal structure alone can be regarded as a measure of attractive interaction or whether the proximity of two atoms is a byproduct induced by other superordinate structure principles like the formation of a tetrameric aggregate.

Table 3-1. Selected bond lengths [pm] and angles [ $\left.{ }^{\circ}\right]$ for 2-AnisolLi.pmdeta (1) and related compounds

\begin{tabular}{|c|c|c|c|c|}
\hline & $\begin{array}{l}\text { 2-AnisolLi·pmdeta } \\
\text { (1) }\end{array}$ & Theory & $\begin{array}{c}{[1 \text {-lithio-2- }} \\
\text { methoxybenzene }]_{8} \cdot \text { tmeda }^{[94]}\end{array}$ & $\begin{array}{l}\text { 1-lithio-2- } \\
\text { methoxybenzene }\end{array}$ \\
\hline Li1-C1 & $210.9(3)$ & 208.69 & 229.5 (av.) & 225.2 (av.) \\
\hline Li1-N1 & $214.0(3)$ & 222.70 & 213.6 & - \\
\hline Li1-N2 & $214.4(3)$ & 220.39 & - & - \\
\hline Li1-N3 & $215.0(3)$ & 222.35 & - & - \\
\hline Li1-O1 & 296.8(3) & 312.17 & 195.0 & 195.5 (av.) \\
\hline C1-Li1-N1 & $117.37(14)$ & 117.21 & - & - \\
\hline C1-Li1-N2 & $133.11(15)$ & 133.12 & - & - \\
\hline C1-Li1-N3 & $114.90(14)$ & 114.91 & - & - \\
\hline
\end{tabular}

\subsubsection{Computational studies}

To resolve the question of an attractive interaction between oxygen and lithium, as proposed in the literature, terminally, quantum chemical calculations have been carried out. These were followed by the application of two tools for bonding analysis, QTAIM and ELI, that have been introduced in the previous chapter.

Starting from the crystal coordinates, a gas phase optimization with the Turbomole ${ }^{[59]}$ program package on the B3-LYP ${ }^{[64-66]}$ level employing the Ahlrich's TZVP ${ }^{[97]}$ basis set was carried out. The resulting wavefunction was exported to molden format with the tool tm2molden. The molden files were then read in by the DGRID-4.4 ${ }^{[78]}$ program of M. Kohout. Thereon the scalar fields of the ED, the Laplacian and ELI have been calculated on a grid with a mesh size of $0.1 \mathrm{Bohr}$ and a border size around the molecule of 5.0 Bohr. Generally even smaller mesh sizes are preferable, but since the size of a mesh scales with $\mathrm{N}^{3}$, reducing the mesh size to $0.5 \mathrm{Bohr}$ would increase the grid by the factor 8 . The integral of the total electron density over the grid was 157.54 e (total number of electrons in 1 is 158). 
This is due to the compromise for the mesh size and because the electron density is cropped at some level by the grid border. However, the qualitative predictions made by QTAIM and ELI remain intact. The resulting grid files were analyzed in a subsequent step by the basin search program BASIN-4.4.

To identify the coordination number of lithium in 1, a topological analysis of the ED according to QTAIM was carried out. Within QTAIM, the coordination number of an element is simply given by the number of bond paths that originate from it. The result of the analysis yielded all atomic basins (51) as well as all anticipated BCP between them. The molecular graph obtained is represented in figure $3-5$.

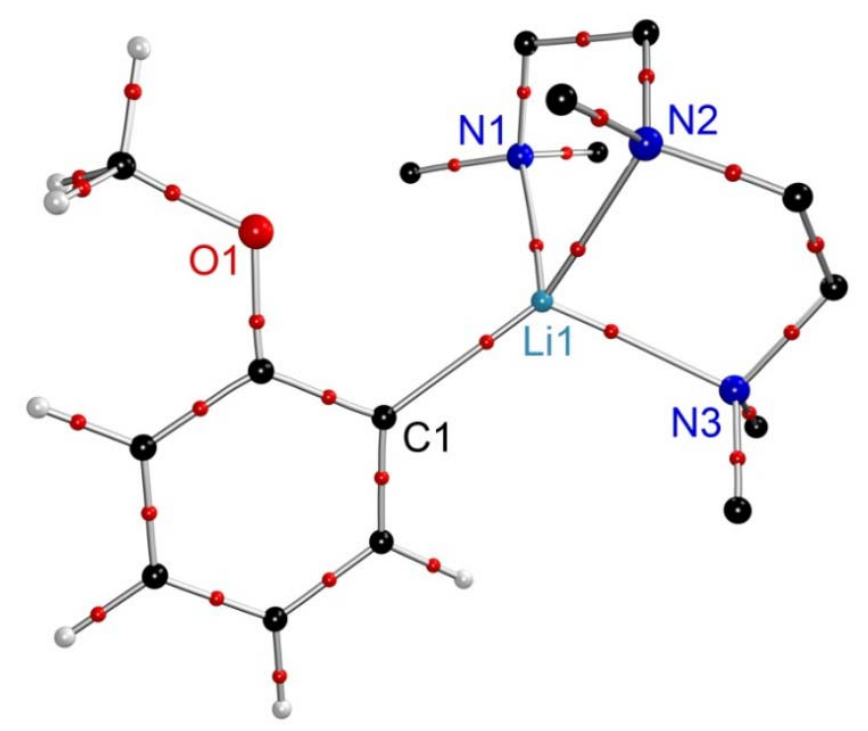

Figure 3-5. Molecular graph of 2-AnisolLi·pmdeta (1).

The situation around lithium is the following: There are four bond paths from the lithium atom to $\mathrm{C} 1$ as well as to all nitrogen atoms of the donor base pmdeta. Although the QTAIM method is a very sensitive tool, no BCP between $\mathrm{O} 1$ and Li1 were detected. Lithium is tetracoordinate and threefold pmdeta coordination seems to be favorable over intramolecular side arm coordination of the methoxy group as suggested by Harder et al. ${ }^{[93]}$ The three nitrogen donors alone saturate the electron deficiency at the lithium cation. All three $\mathrm{N}-\mathrm{Li}$ bonds can be regarded as equal, although the density at the BCP of the N2-Li bond $\left(0.13 \mathrm{e} \AA^{-3}\right)$ seems to be a little higher than at the BCPs between lithium and the outer $\mathrm{NMe}_{2}$ groups $\left(0.12 \mathrm{e} \AA^{-3}\right)$. The Laplacian at these BCPs, which are located close to the lithium atom, is positive which is in agreement with a strong polar donor bond. The electron density values at the $\mathrm{BCP}$ between carbon and lithium is with $0.23 \mathrm{e} \AA^{-3}$ significantly higher compared to the values of the $\mathrm{Li}-\mathrm{N}$ donor bonds, but it has to be mentioned, that in the optimized gas phase structure the $\mathrm{C} 1-\mathrm{Li} 1$ bond length is much shorter than the $\mathrm{N}-\mathrm{Li}$ donor 
bonds (on av. by $12 \mathrm{pm}$ ). In the X-ray experiment the carbon-lithium and the lithium-nitrogen distances differ only by $4 \mathrm{pm}$.

Table 3-2. Electron densities $\left[\mathrm{e} \AA^{-3}\right]$, Laplacians $\left[\mathrm{e}^{-5}\right]$, and ellipticities at the BCPs of the $\mathrm{Li}-\mathrm{C}$ and Li$\mathrm{N}$ bonds

\begin{tabular}{c|ccc}
\hline & $\rho\left(\mathbf{r}_{\mathrm{BCP}}\right)$ & $\nabla^{2} \rho\left(\mathbf{r}_{\mathrm{BCP}}\right)$ & $\varepsilon\left(\mathbf{r}_{\mathrm{BCP}}\right)$ \\
\hline C1-Li1 & 0.23 & 3.57 & 0.04 \\
N1-Li1 & 0.12 & 2.59 & 0.09 \\
N2-Li1 & 0.13 & 2.78 & 0.00 \\
N3-Li1 & 0.12 & 2.63 & 0.09 \\
\hline
\end{tabular}

The strong polarity of the carbon-lithium bond is not only evident from the values at the BCP but also from the integrated QTAIM charges over the corresponding atomic basins. The atomic charge of lithium of $+0.88 \mathrm{e}$ is close to the loss of a whole electron which is a definite sign for the predominant ionic character of the bond. This is in accordance with earlier QTAIM studies, which assigned positive values between 0.87-0.93 e to the lithium cation. ${ }^{[98,99]}$

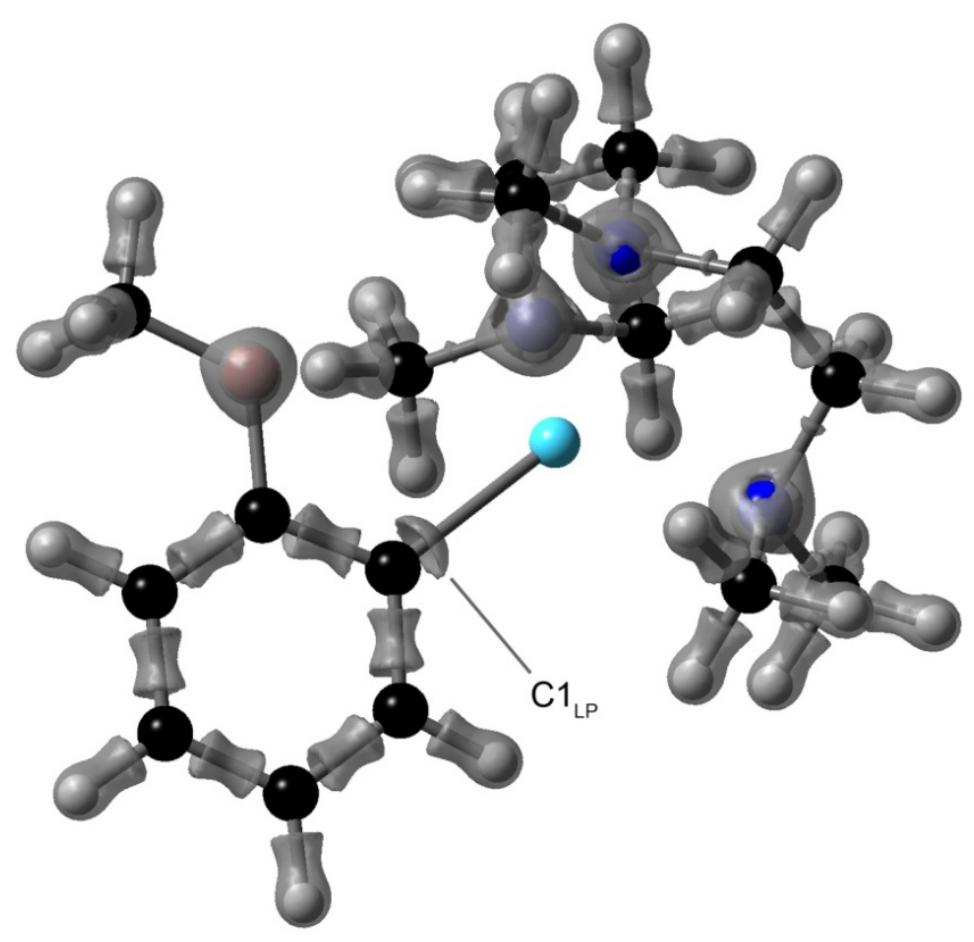

Figure 3-6. Laplacian isosurface representation of 1 at $-5.30 \mathrm{e}^{-5}$.

Figure 3-6 shows an isosurface of $\nabla^{2} \rho$ at -5.30 e $\AA^{-5}$. At this negative level charge accumulations can be made visible and the carbanionic lone pair density pointing in the 
direction of the lithium atom can be seen. The electron density at the nitrogen atoms is slightly polarized towards the lithium cation which is indicative of a dative polar bond. However, against chemical intuition no interaction between the lone pair densities of the methoxy group with the lithium atom can be observed.

As QTAIM results about the existence or absence of chemical bonds have been discussed controversial in the literature over the last years, ${ }^{[0,100,101]}$ the situation was also parsed from the ELI perspective. Especially the location of the lone pair basins on the oxygen atom should provide an indication of an oxygen-lithium donor bond. In agreement with Bader's theory the ELI analysis yields a disynaptic basin between C1 and Li1 as well as three disynaptic basins between the pmdeta nitrogen atoms and Li1. While the C-Li basin contains 2.16 e and has a volume of $152.46 \AA^{3}$, the $\mathrm{N}-\mathrm{Li}$ basins contain only 1.94 e. Due to the high electronegativity the ELI-D maximum of the disynaptic $\mathrm{N}-\mathrm{Li}$ basins is closer to nitrogen than to lithium and the basins are very compact with a volume of only $42-50 \AA^{3}$. In accordance with QTAIM results, no O1-Li1 interaction can be identified. The oxygen lone pairs are manifested by monosynaptic oxygen basins that are not polarized towards the electron deficient lithium atom and contain approximately 2.4 electrons each. Figure 3-7 shows selected ELI basins on an ELI level of 1.4.

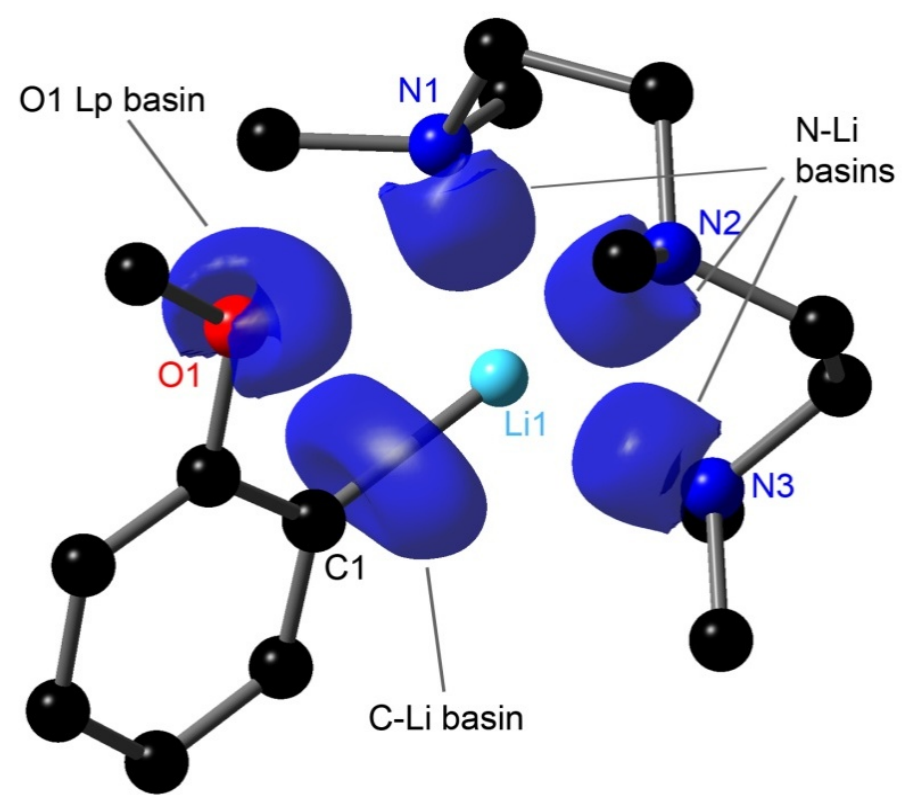

Figure 3-7. Isosurface representation of selected ELI basins of 1 on an ELI level of 1.4. 
Table 3-3. ELI-D properties for the disynaptic Li-C and Li-N basins and the monosynaptic oxygen basins in 1. Distances in $[\mathrm{pm}]$ and Volumes in $\left[\AA^{3}\right]$.

\begin{tabular}{|c|c|c|c|c|c|c|}
\hline & ELI-D max & $\begin{array}{c}\rho(\mathbf{r}) \\
\text { Integral }\end{array}$ & ECCNT & $\begin{array}{c}\text { Distance from } \\
\text { A }\end{array}$ & $\begin{array}{c}\text { Distance from } \\
\text { B }\end{array}$ & Volume \\
\hline C1-Li1 & 2.1620 & 2.47 & 0.033 & 86.8 & 121.7 & 152.46 \\
\hline N1-Li1 & 1.9365 & 2.33 & 0.257 & 75.1 & 148.1 & 49.90 \\
\hline N2-Li1 & 1.9394 & 2.30 & 0.107 & 73.6 & 142.9 & 42.29 \\
\hline N3-Li1 & 1.9484 & 2.32 & 0.172 & 74.6 & 148.2 & 47.34 \\
\hline O1 Lp & 1.7228 & 2.45 & - & 59.3 & - & 40.79 \\
\hline O1 Lp & 1.7182 & 2.38 & - & 58.7 & - & 42.58 \\
\hline
\end{tabular}

\subsubsection{Conclusion}

While rate studies of Collum et al. indiciate that the importance of lithium-oxygen interactions in a pre-metalation complex may be overrated to explain the observed ortho

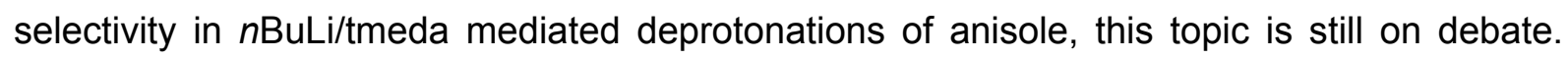
However, this stabilizing interaction is also postulated to exist in the ortho-lithiated intermediate, which is formulated as a monomer in many textbooks of organic chemistry.

By determining the first crystal structure of a monomeric lithiated anisole and subsequent computational studies a clear statement about such a stabilizing effect in the ortho-metalated intermediate can be given. Both QTAIM and ELI results coincidence in that no oxygenlithium donor bond in $\mathbf{1}$ is present. Even if the only bidentate ligand tmeda is employed, one should be careful to postulate a lithium-oxygen interaction, since these compounds are not monomeric in solid state and solution but seek stabilization in aggregation. The orientation of a functional group in such an oligomer can be dictated by various steric factors also. Moreover, it has to be emphasized that short bond distances derived from X-ray structure determinations alone are not necessarily a sign for an attractive interaction. 


\subsection{Scavenger}

\subsubsection{Introduction}

The tendency of organolithium reagents to form oligomeric aggregates of decreased reactivity and solubility in apolar solvents makes it necessary to activate them for deprotonation reactions. ${ }^{[102,103]}$ The goal is to break up the oligomers to produce smaller, more reactive dimers or even monomers. ${ }^{[104]}$ This is normally achieved by addition of neutral donor ligands like tmeda or dme or the addition of co-complexing salts. Intramolecular side arm complexation also can result in lower aggregation grades. ${ }^{[105]} \mathrm{A}$ special case is the activation of organolithiums with another organometallic species like a metal alkoxide. Since solvation of the lithium cation should increase with the basicity of the donor base, the addition of lithium alkoxides should accelerate metalation reactions in particular. In addition, the thereby formed mixed species can have a completely different regioselectivity. Evidence for the formation of mixed aggregates was found by NMR spectroscopy in solution and by Xray structure determination in the solid state. Hereby it was found that $n \mathrm{BuLi} / \mathrm{LiOnBu}$ is tetrameric in thf. ${ }^{[106]}$ In the solid state a donor free form of $n \mathrm{BuLi} / \mathrm{LiOtBu}$ has been characterized (figure 3-8). ${ }^{[107]}$ Lobomir et al. found that the stability of these mixed species is biggest if the substituent at the alkoxide is large while the substituents on the organolithium reagent are small. ${ }^{[108]}$ The reason could be that in sterically demanding alkoxides the formation of supermolecular aggregates is hindered and adduct formation with organolithium compounds is expedient.

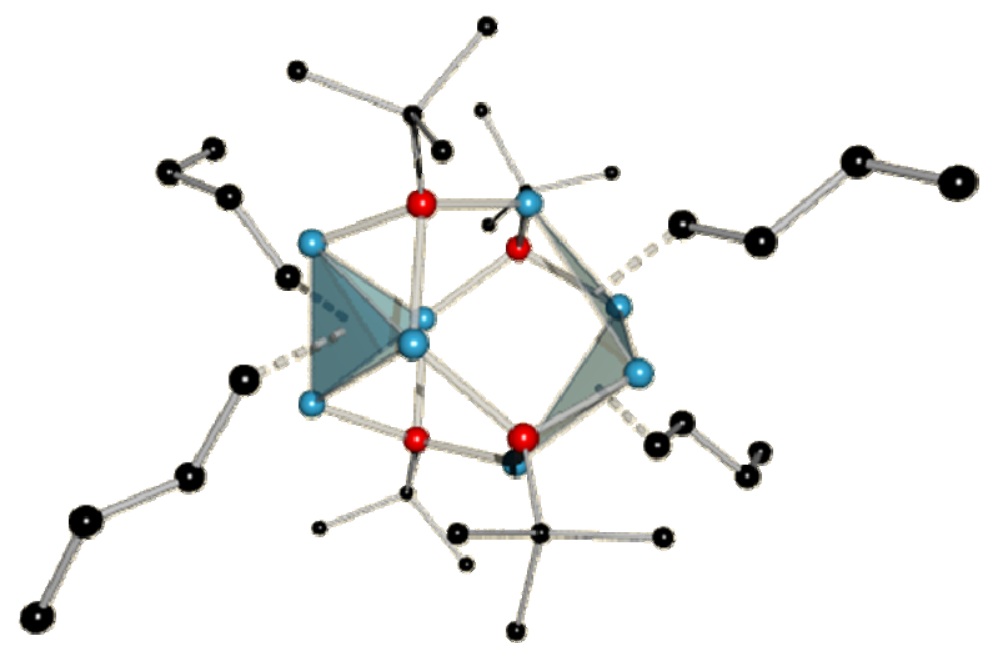

Figure 3-8. Molecular structure of a mixed aggreagate from $n \mathrm{BuLi}$ and $\mathrm{Li}-\mathrm{tBuOLi} .{ }^{[107]}$ 
In practice the alkoxides of heavier alkali metals like sodium and potassium are even stronger activators than lithium alkoxides. In this case in addition to initial adduct formation a metal interchange of lithium and the heavier alkali metal can take place leading to the formation of highly reactive alkyl sodium or alkyl potassium species. The most impressive activation of this kind was found by Lochmann and Schlosser. ${ }^{[103]}$ They could show, that the addition of one equivalent potassium tert-butoxide to a solution of $n$-butyllithium improves the reactivity so dramatically, that they used the term "superbase" or LICKOR base for these new types of mixed-metal reagents. ${ }^{[109]}$ In contrast to conventional organolithium reagents LICKOR bases deprotonate benzene or toluene easily. The identity of the reactive species and the reaction mechanism of super bases is still an object of controversial debate. Opposite to the monometallic system $n \mathrm{BuLi} / \mathrm{LiO} n \mathrm{Bu}$, for the LICKOR superbase NMR studies do not provide the existence of a mixed aggregate. ${ }^{[110]}$ It has also been suggested that the active species in superbase deprotonations is a pure organo potassium compound.

Related to this class of compounds are Mulvey's inverse crown ethers. ${ }^{[111]}$ Their fascinating structure motif can be formed when certain alkali metal amides are mixed with certain zinc or magnesium bisamides. Classical crown ethers are polydentate ligands, in which Lewis basic oxygen atoms are linked via ethylene bridges to form a macrocycle. This cryptand is able to coordinate a variety of metal atoms, especially the ones of the alkali and earth alkali group. In contrast, inverse crown ethers consist of a ring system which is built up of Lewis acidic alkali metals which are bridged by amide ligands. Therefore they are able to solvate anions (figure 3-9).
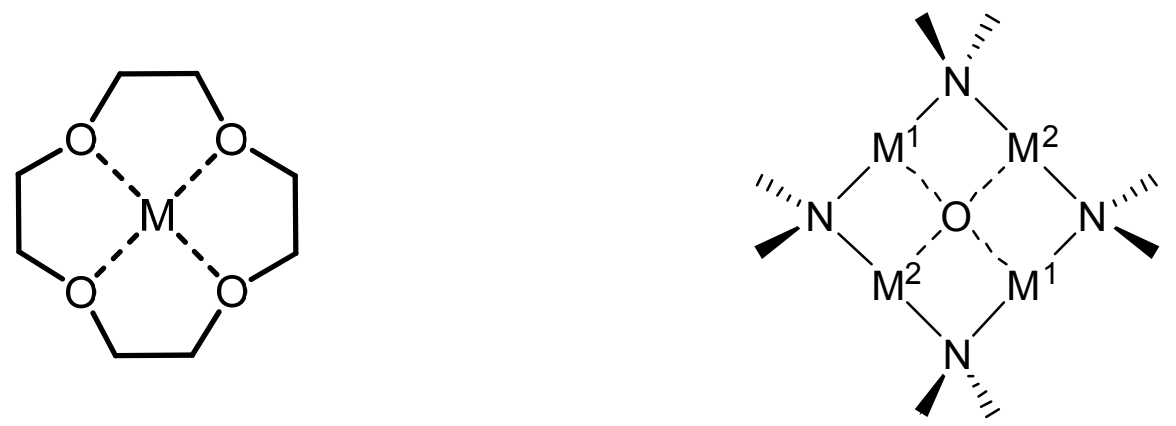

Figure 3-9. Classical crown ether (left) and inverse crown ether (right).

The environment inside the inverse crown is even able to solvate dianionic species. This is demonstrated by the twofold deprotonation of benzene. Hereby, not the strength of the base is the crucial factor but the stabilization of two negative charges by $\sigma$-interactions to magnesium and additional $\pi$-interactions to sodium (scheme 3-5). ${ }^{[12]}$ 


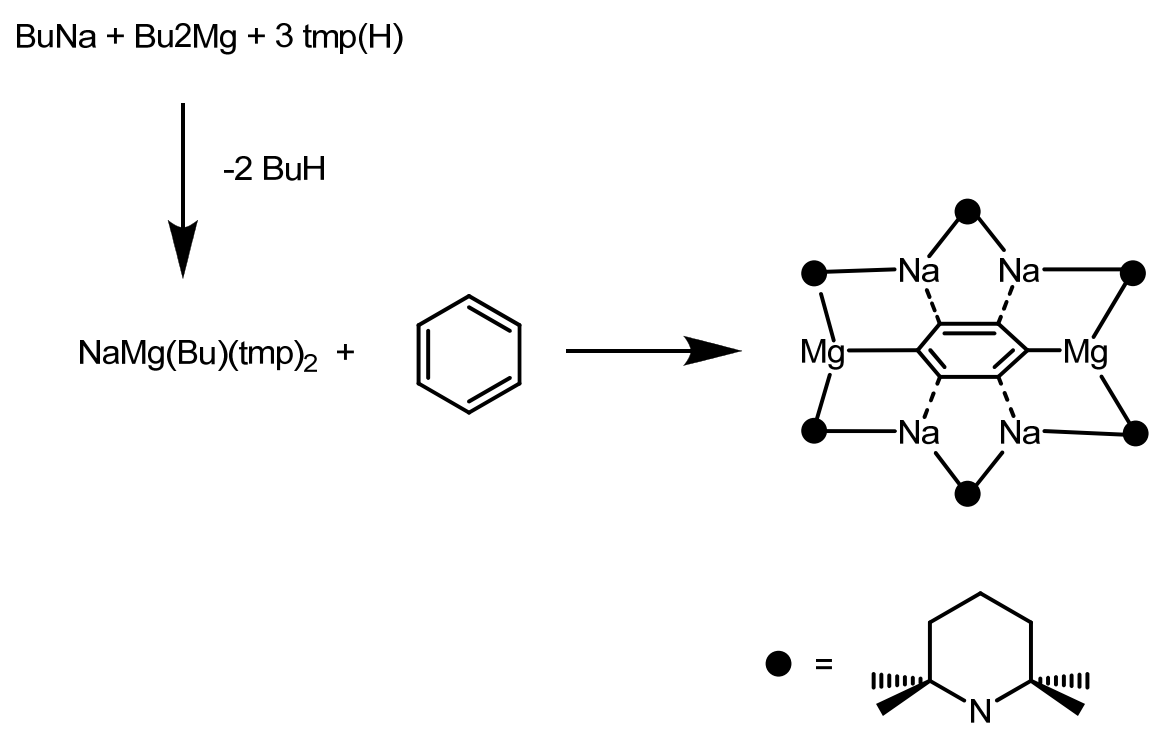

Scheme 3-5. Twofold deprotonation of benzene by a mixed metal reagent and encapsulation of the dianion in an inverse crown ether. ${ }^{[112]}$

No mixed complexes between the smallest organolithium methyllithium and lithium alkoxides have been reported so far. This is surprising since Lobomir et al. stated that the stability of these mixed species is highest if the substituent at the alkoxide is large while the substituents on the organolithium reagent are small. ${ }^{[108]}$ Since the instability of these compounds is a limiting factor for the isolation, the preparation of the mixed reagent MeLi/LiOtBu seemed to be a promising target.

\subsubsection{Preparation}

tert-butanol was reacted with two equivalents of methyllithium in diethyl ether at $0^{\circ} \mathrm{C}$ and stirred for $1 \mathrm{~h}$ (scheme 3-6). In the first step, the alcohol is deprotonated. In principle the resulting alkoxide should be able to complex the second equivalent of methyllithium to form a mixed aggregate. After storing the flask one week at $5{ }^{\circ} \mathrm{C}$, colorless crystals that were suitable for X-ray analysis were obtained.

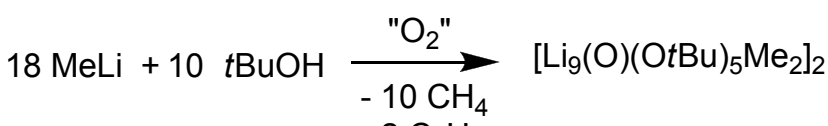

$$
\begin{aligned}
& \text { - } 2 \mathrm{C}_{2} \mathrm{H}_{6}
\end{aligned}
$$

Scheme 3-6. Reaction of MeLi with $t \mathrm{BuOH}$.

Surprisingly, the crystal structure of $\mathbf{2}$ consists of four methyllithium-, ten lithium tertbutoxide and two $\mathrm{O}^{2-}$ ions. Presumably, molecular oxygen was reduced and encapsulated in a three dimensional inverse crown cage. Reactions of organolithiums with molecular oxygen have observed before and a radical mechanism has been proposed. ${ }^{[113]}$ Thereby molecular 
oxygen inserts into an $\mathrm{O}-\mathrm{Li}$ bond forming a peroxide $t \mathrm{Bu}-\mathrm{O}-\mathrm{OLi}$. This peroxide itself can react with another tert-butyllithium molecule to give lithium-tert-butoxide.

The asymmetric unit of $\mathbf{2}$ contains only half of the molecule. The second part is generated by an inversion center. The two encapsulates $\mathrm{O}^{2-}$ anions $\mathrm{O} 1$ and $\mathrm{O}^{\prime}$ are sevenfold coordinated by lithium cations (figure 3-10).

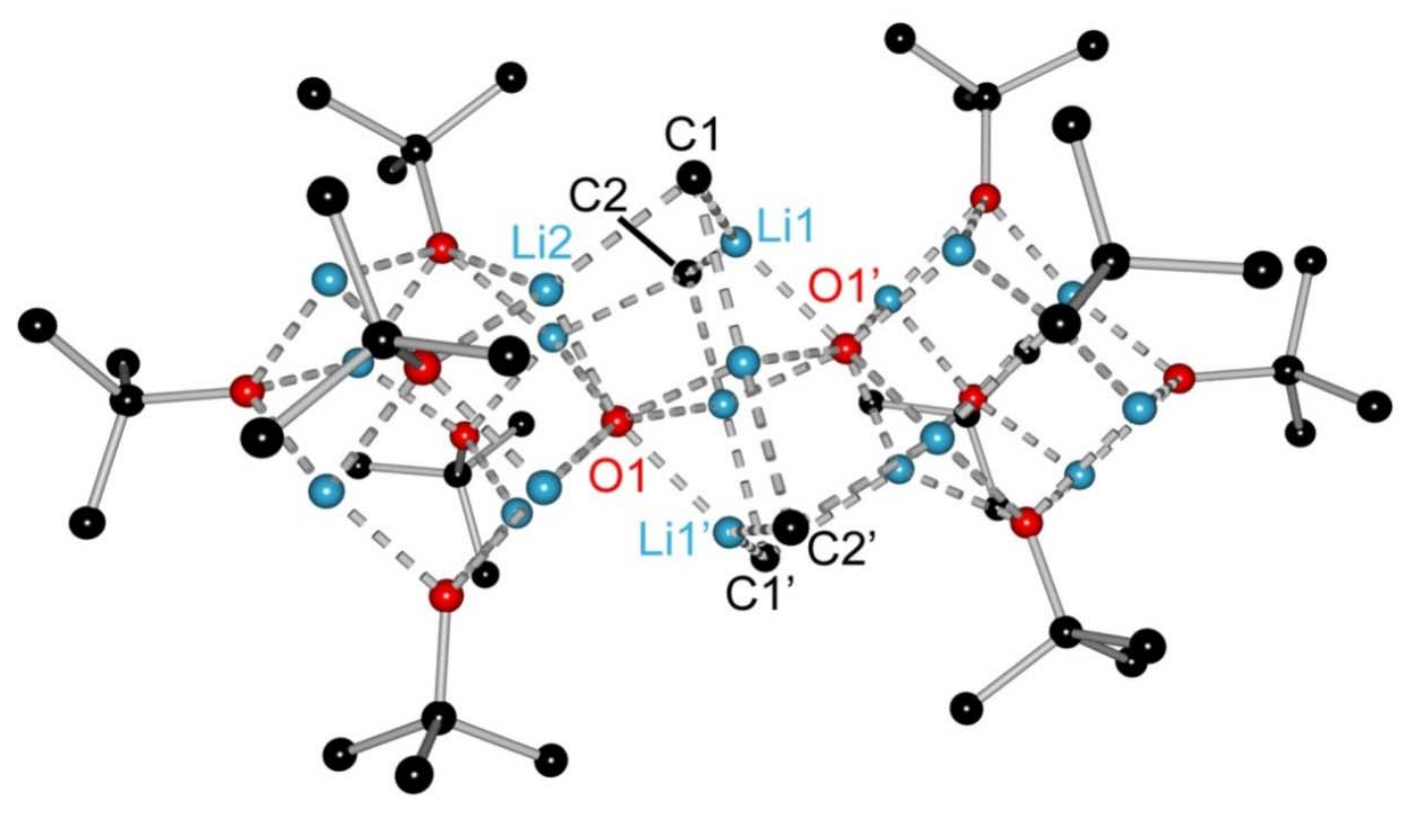

Figure 3-10. Molecular structure of scavenger (2).

The structure can be understood with the aid of the ring stacking and laddering principle known from lithium amides. ${ }^{[14]}$ This model can easily be extended to the aggregation of lithium alkoxides. Scheme 3-7 shows the ladder association of six lithium tert-butoxide units which after cyclization form a hexamer.

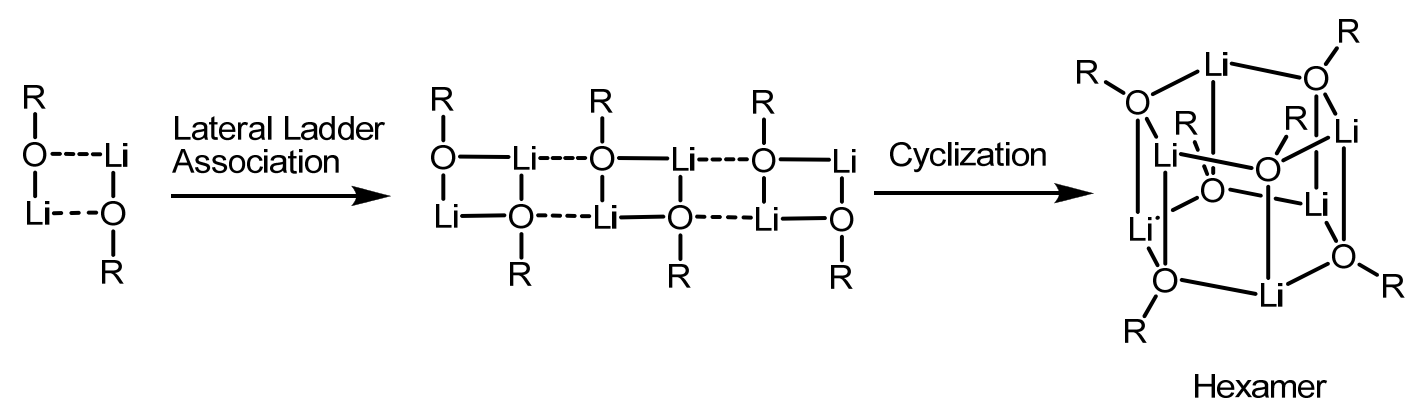

Scheme 3-7. Stacking principles of lithium alkoxides.

Although lithium tert-butoxide has been proposed to be hexameric in apolar solvents from NMR, cryoscopy and mass spectrometry, ${ }^{83}$ it was until 2002 that its crystal structure was reported by Nekola et al. ${ }^{[115]}$ Indeed, the solid state structure of lithium tert-butoxide is in compliance with the solution structure obtained as an octahedron $(\mathrm{BuOLi})_{6}$. Interestingly, a novel octameric modification of $t \mathrm{BuOLi}$ has been published shortly after. ${ }^{[116]}$ 
To rationalize the structure building principle of 2 , hexameric $(t \mathrm{BuOLi})_{6}$ is a good starting point. Normally, these organometallic clusters are described as lithium atoms formally building a polyeder which triangular faces are capped by anions. In the following case it is convenient to switch the perspective and connect the six oxygen atoms to an octahedron. Then in 2 formally one vertex of $(t \mathrm{BuOLi})_{6}$ has been replaced by an $\mathrm{O}^{2-}$ anion. This encapsulated anion is bridged by lithium contacts of a spacer consisting of four methyllitium units to a second identical octahedron (scheme 3-8). Interestingly, a similar structural motif can be found in the related $(\mathrm{tBuOLi})_{6} \cdot(\mathrm{LiOH})_{6}$, that was reported by Lambert et al. ${ }^{[117]}$ In their case the structural components of the $(t \mathrm{BuOLi})_{6}$ octahedron remains unaltered also and the connection of two such polyeders is achieved by bridging $\mathrm{LiOH}$ units.

$2(t \mathrm{BuOLi})_{6}$

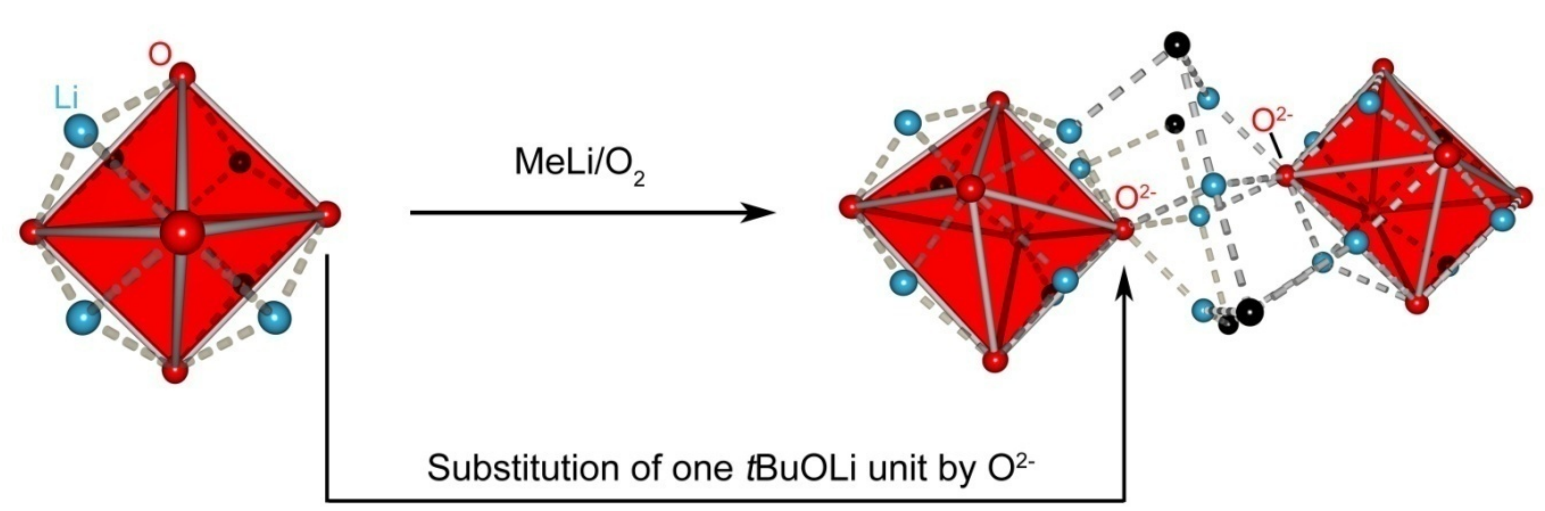

Scheme 3-8. Formal transition of a hexameric $t \mathrm{BuOLi}$ cluster to 2. Carbon atoms of $t \mathrm{Bu}$-groups have been omitted for clarity.

The bond lengths of the Li-O contacts vary between 185.5 and 206.2 pm (table 3-4). In comparison, $\mathrm{Li}-\mathrm{O}$ bond distances for $(\mathrm{BuOLi})_{6}$ have been reported to be in a range between 186.1 and 198.4 pm. ${ }^{[115]}$ However, because of severe disorders in the structure, no explicit bond lengths and angles with estimated standard deviations have been published. The octameric form of lithium tert-butoxide does not suffer from such problems and the bond distances here were determined to be within 188.9 and $196.8 \mathrm{pm} .{ }^{[118]}$ 


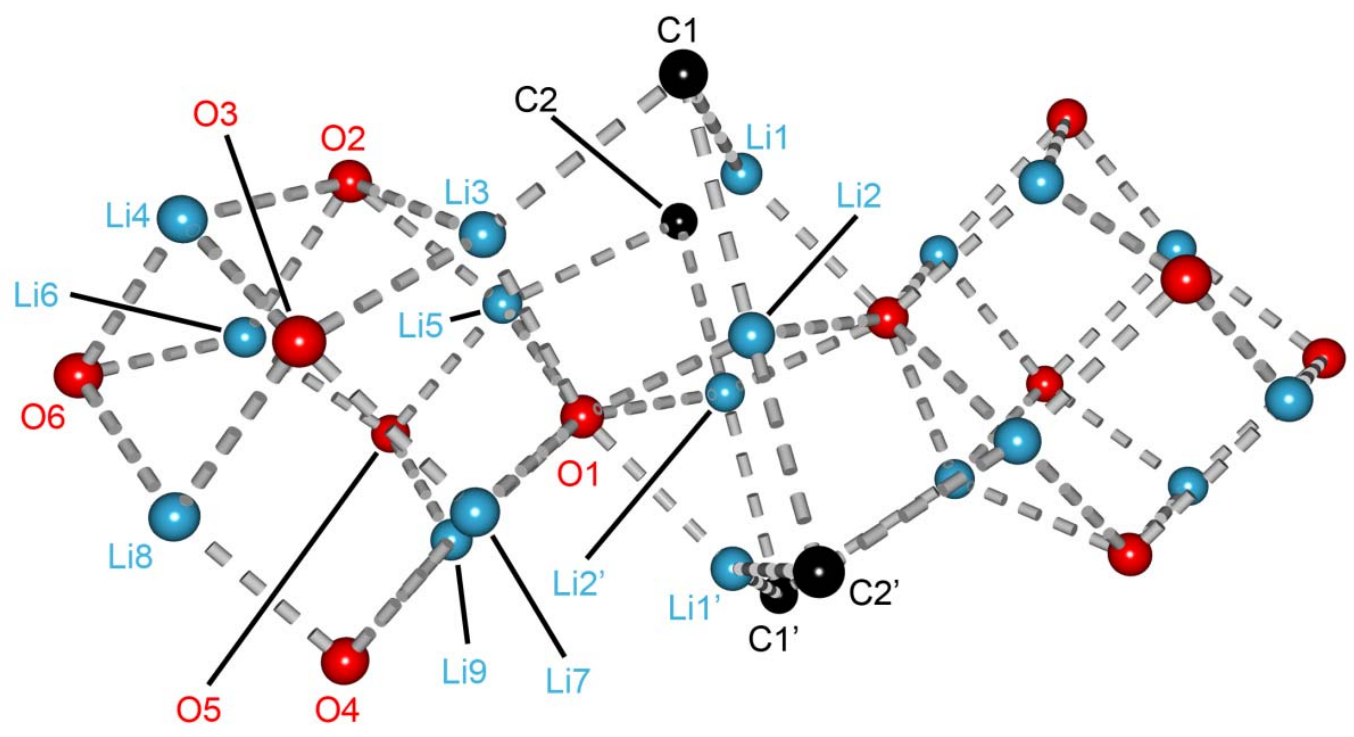

Figure 3-11. Structure 2 displaying the lithium-oxygen core and the bridging methyllithium units.

The reason for the large distribution of lithium-oxygen bond lengths in 2 originates from the existence of three types of $\mathrm{Li}-\mathrm{O}$ bonding modes (figure 3-12, right side). $\mathrm{O} 1$ is in an exceptional position since it is the captured ion. It possesses no attached tBu-group, is doubly negative charged and features mainly electrostatic interactions to the surrounding lithium cations. The additional charge resulting from the replacement of a $t \mathrm{BuO}^{-}$group by $\mathrm{O}^{2-}$ is counterbalanced by a seventh lithium cation (Li3) which sits on top of a $\mathrm{Li}_{6} \mathrm{O}_{6}$ chair (figure 3-9, left side). The Li-O contacts from 01 with a mean value of $196 \mathrm{pm}$ are at the head of lithium-oxygen bond lengths. O1-Li5 is the longest distance with a value of $206.2 \mathrm{pm}$ reflecting the geometrically distorted coordination sphere of $\mathrm{O} 1$.
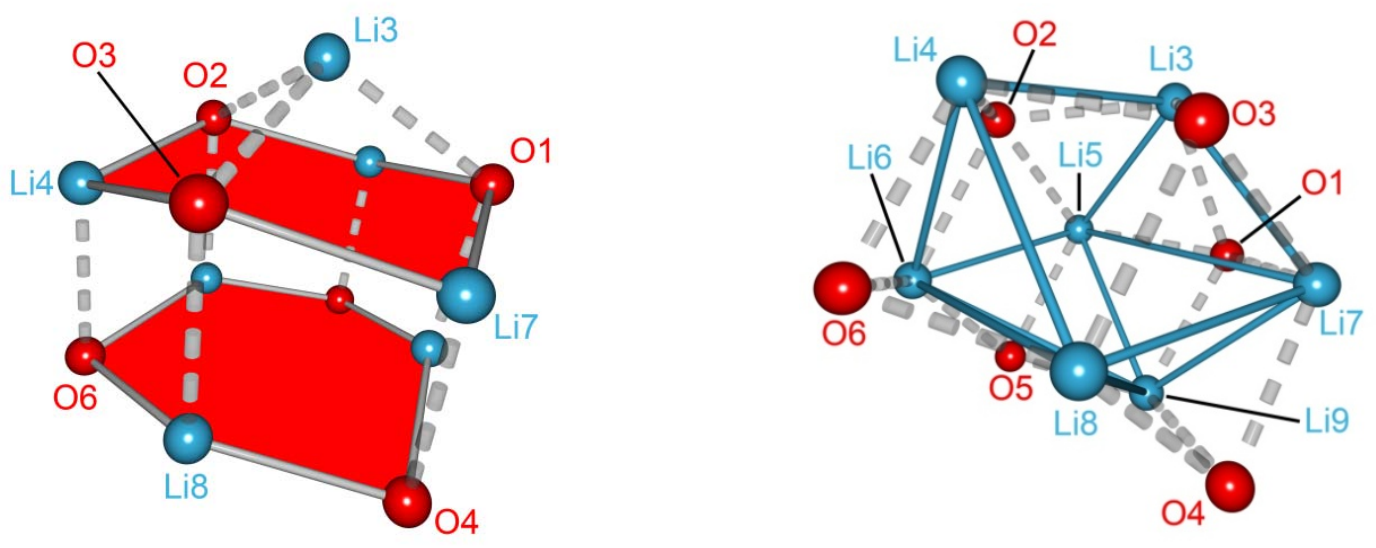

Figure 3-12. Chair motif (left) and oxygens capping triangular and quadratic lithium faces (right).

The remaining $t \mathrm{BuO}-\mathrm{Li}$ contacts can be divided into two classes. While $\mathrm{O} 2$ and $\mathrm{O} 3$ are capping quadratic faces and thus are linked to four lithium atoms, O4, $\mathrm{O} 5$ and $\mathrm{O} 6$ show the 
same coordination behavior like in the deltaeder $(\mathrm{tBuOLi})_{6}$, forming three $\mathrm{Li}-\mathrm{O}$ bonds each. Hence the average bond length between $\mathrm{O} 2 / \mathrm{O} 3$ and lithium (av. $198.1 \mathrm{pm}$ ) is prolonged compared to the Li-O contacts with O4/O5/O6 (av. 189.4 pm).

The methanide ions $C 1$ and $C 2$ are sitting on top of the classical lithium triangle. This bonding mode is the most prominent in organolithium chemistry, characterizing the building principle of most higher polyhedric oligomers. The mean carbon-lithium distance is $224.35 \mathrm{pm}$ and thus comparable to that in pure organolithium clusters. ${ }^{[119,120,12]}$ However, the positions of $\mathrm{C} 1$ and $\mathrm{C} 2$ with respect to the lithium faces are not identical. Whereas $\mathrm{C} 1$ is located nearly above the center of $\mathrm{a} \mathrm{Li}_{3}$ triangle and the associated lithium-carbon distances are the same within 5 pm, C2 exhibits two short (220.2(8) and 225.8(2) pm) and one notably longer (231.1(2) pm) Li-C interactions. Such a shift of the $\alpha-C$ away from the center of the $\mathrm{Li}_{3}$ triangle has also been observed in the case of $n \mathrm{BuLi}^{\left[{ }^{[12]}\right.}$

Table 3-4. Selected bond lengths [pm] in Scavenger (2).

\begin{tabular}{l|lllll}
\hline \multicolumn{1}{l}{} & \multicolumn{1}{l}{ X-Ray } & Theory* & & X-Ray & Theory \\
\hline O1-Li5 & $206.2(2)$ & 208.84 & O2-Li3 & $198.1(2)$ & 200.86 \\
O1-Li3 & $197.4(2)$ & 199.39 & O2-Li4 & $192.0(2)$ & 192.4 \\
O1-Li7 & $190.1(2)$ & 189.89 & O2-Li5 & $202.2(2)$ & 206.04 \\
O1-Li9 & $190.5(2)$ & 191.46 & O2-Li6 & $201.5(2)$ & 201.78 \\
O1-Li2 & $197.8(2)$ & 198.19 & O3-Li3 & $202.5(2)$ & 209.16 \\
O1-Li2' & $195.0(2)$ & 197.11 & O3-Li4 & $192.1(2)$ & 192.87 \\
O1-Li1' & $195.2(2)$ & 195.48 & O3-Li7 & $195.3(2)$ & 196.88 \\
C1-Li3 & $223.9(2)$ & 225.43 & O3-Li8 & $201.4(2)$ & 204.69 \\
C1-Li1 & $220.1(3)$ & 220.04 & O4-Li7 & $190.0(2)$ & 190.44 \\
C1-Li2 & $225.2(3)$ & 226.77 & O4-Li8 & $186.7(2)$ & 187.11 \\
C2-Li5' & $231.1(2)$ & 230.60 & O4-Li9 & $195.0(2)$ & 195.65 \\
C2-Li2 & $225.8(2)$ & 226.68 & O5-Li5 & $192.7(2)$ & 195.36 \\
C2-Li1' & $220.0(2)$ & 220.54 & O5-Li6 & $185.9(2)$ & 185.92 \\
& & & O5-Li9 & $189.8(2)$ & 190.03 \\
& & & O6-Li4 & $185.5(2)$ & 187.71 \\
& & & O6-Li6 & $190.1(2)$ & 190.61 \\
& & & O6-Li8 & $188.7(2)$ & 192.36 \\
\hline
\end{tabular}

*B3-LYP/SVP

When discussing the bonds at the lithium centers, it is helpful to recall the building principle of $(t \mathrm{BuOLi})_{6}$. This hexamer can be regarded to be composed of two $\mathrm{Li}_{3} \mathrm{O}_{3}$ stacked rings. The contacts between those two rings are afforded by four-membered $\mathrm{Li}_{2} \mathrm{O}_{2}$ rings. 
Therefore the $\mathrm{O}-\mathrm{Li}-\mathrm{O}$ bond angles can be divided into two sets. Within the $\mathrm{Li}_{2} \mathrm{O}_{2}$ rings, they have a mean value of $100.82(10)^{\circ}$, whereas the O-Li-O bond angles that are part of the sixmembered $\mathrm{Li}_{3} \mathrm{O}_{3}$ chair have a mean value of $133.58(12)^{\circ}$. However, as distinguished from parent $(t \mathrm{BuOLi})_{6}$, in $\mathbf{2}$ the stacking chair is capped by an additional lithium cation. As a result, $\mathrm{Li} 7$ forms on its part $\mathrm{Li}_{2} \mathrm{O}_{2}$ rings with the stacked chair, so the bond angles in this former $\mathrm{Li}_{3} \mathrm{O}_{3}$ ring falls down to $100^{\circ}$.

Table 3-5. Selected experimental bond angles $\left[{ }^{\circ}\right]$ in Scavenger (2).

\begin{tabular}{l|lll}
\hline O1-Li3-O2 & $104.67(10)$ & O5-Li5-O1 & $98.40(9)$ \\
O1-Li3-O3 & $98.76(9)$ & O5-Li5-O2 & $98.06(10)$ \\
O1-Li7-O3 & $103.95(10)$ & O5-Li6-O2 & $100.59(10)$ \\
O1-Li9-O4 & $100.84(10)$ & O5-Li6-O6 & $132.60(12)$ \\
O2-Li3-O3 & $97.14(9)$ & O5-Li9-O1 & $105.18(10)$ \\
O2-Li4-O3 & $102.88(11)$ & O5-Li9-O4 & $133.37(12)$ \\
O2-Li5-O1 & $100.09(9)$ & O6-Li4-O2 & $103.16(10)$ \\
O4-Li7-O1 & $102.85(10)$ & O6-Li4-O3 & $102.00(10)$ \\
O4-Li7-O3 & $100.85(10)$ & O6-Li6-O2 & $98.04(10)$ \\
O4-Li8-O3 & $99.78(10)$ & O6-Li8-O3 & $97.52(10)$ \\
O4-Li8-O6 & $134.78(12)$ & & \\
\hline
\end{tabular}

\subsubsection{Computational Studies}

The chemistry of anion solvating inverse crown ethers can be regarded as exceptional. Already the building principles in simple organolithium compounds, lithium alkoxides or lithium amides are multi-faceted, but the formation of complexes with mixed composition and their ability to capture anions add yet another crucial aspect. While bonding concepts for the class of lithium reagents have been developed, the nature of interactions between solvated ions and Lewis acidic alkali metals is much less discovered. Furthermore, bond properties for lithium bonded to $\mathrm{O}^{2-}$ are interesting to compare with bonds of lithium to alkoxides and neutral oxygen containing donor bases since the basicity of the oxygen atom plays an important role in the deaggregation process of organolithiums.

The first observation from the X-ray structure analysis yields seven neighboring lithium cations for the encapsulated oxygen anion. But it must be noted that the lithium-oxygen contacts differ in length considerably (nearly $20 \mathrm{pm}$ ) making it difficult to state the exact coordination number and compare the different Li-O interactions. Bader's QTAIM is able to identify bonds between atoms beyond a discussion soley based on atomic distances. Consequently, theoretical calculations on the B3-LYP/SVP level of theory were carried out to 
obtain the ED for a topological analysis. After a gas phase optimization values for $\rho(\mathbf{r})$ and $\nabla^{2} \rho(\mathbf{r})$ were calculated on a grid with 0.1 Bohr mesh size. Since 1 is a very huge molecule and grid size scales by the factor $\mathrm{N}^{3}$, the grid was calculated for a cube containing the central spacer unit and the solvated oxygen anions.

During the topological analyis (figure 3-13) bond critical points on all seven 01-lithium trajectories could be found. Furthermore, the carbanions develop three bond paths to lithium atoms which reflects the prominent $\mu_{3}$ capping motif found in other lithium organics.

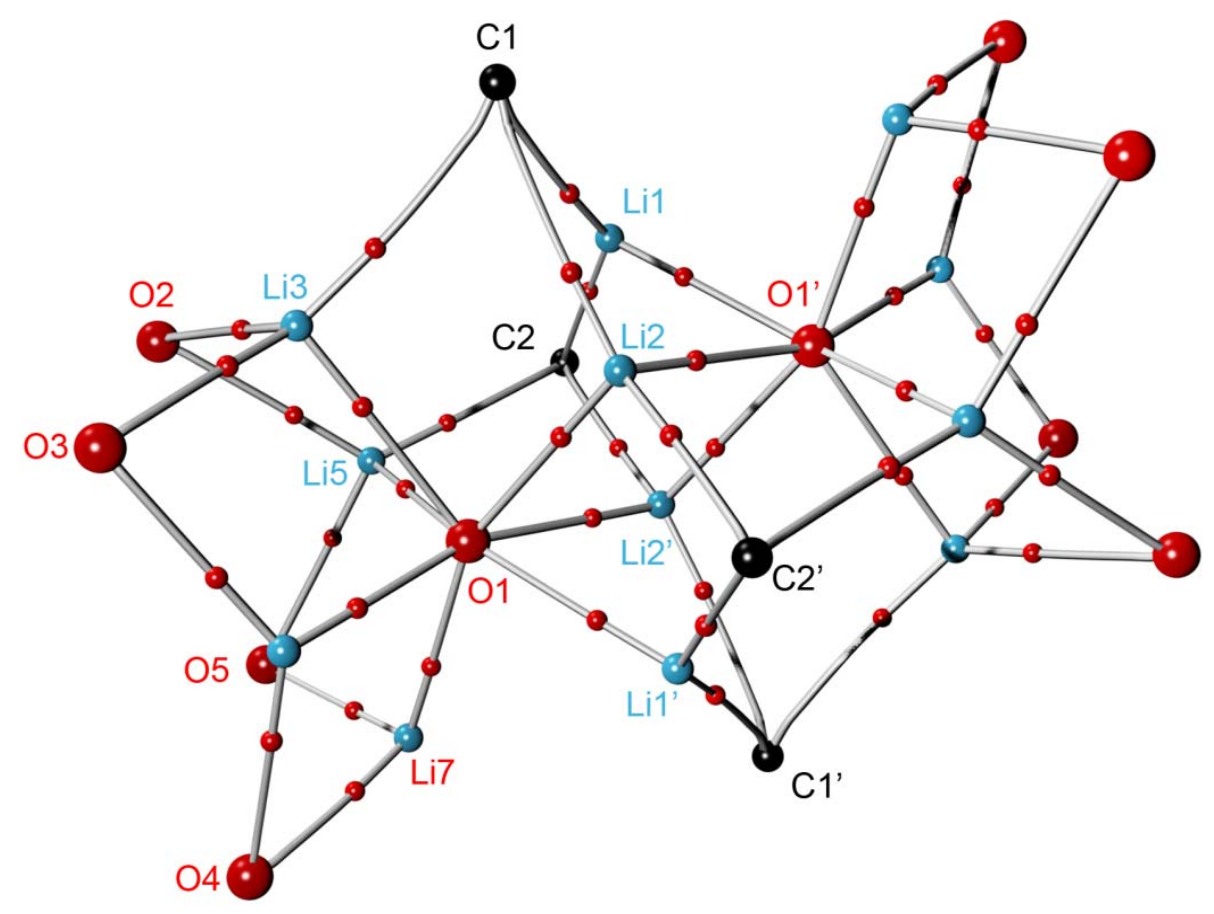

Figure 3-13. Molecular graph of scavenger (2).

The values for $\nabla^{2} \rho(\mathbf{r})$ of all investigated BCPs between lithium and oxygen or carbon atoms are positive. This is in accordance to the anticipated strong polar bonding, thus all BCPs are shifted towards the more electropositive element and situated in a region of charge depletion. The density values at the critical points between $\mathrm{O} 1$ and the coordinating lithium atoms vary between 0.16 and $0.25 \mathrm{e}^{-3}$. The lowest value is obtained for O1-Li5 while the highest value is found for $\mathrm{O} 1-\mathrm{Li} 7$. This directly corresponds to the respective bond distances, reflecting that the shortest O1-Li7 bond with 189.89 pm (X-ray 190.1(2)) is also the strongest and the extremely long O1-Li7 interaction with 208.84 pm (X-ray 206.2(2)) is much weaker. The density at $\mathrm{BCP}_{\mathrm{O} 3-\mathrm{Li} 3}\left(0.19 \mathrm{e}^{-3}\right)$ can be regarded as an internal standard for lithium oxygen interaction in lithium alkoxides. The differences between an $\mathrm{O}^{2-}$-lithium interaction and an $\mathrm{O}^{1-}$-lithium interaction are not as pronounced as could be expected from uncoupled electrostatic considerations. However, the high coordination number of $\mathrm{O} 1$ has to be taken 
into account, too. Therefore the additional charge is shared between seven lithium atoms, which on average leads just to a slight increase of electron density at each BCP.

The fact that the methanide ions $\mathrm{C} 1$ and $\mathrm{C} 2$ cap the triangular lithium faces in an asymmetric fashion has been mentioned in the structural discussion. In fact, these differences are reflected by the values of the respective BCPs between carbon and lithium, too. Both BCPs which correspond to the short carbon-lithium contacts C1-Li1 and C2-Li1' reveal significantly higher values for $\rho(\mathbf{r})\left(0.16 \mathrm{e} \AA^{-3}\right.$ each) than the corresponding values for the elongated carbon-lithium bonds (av. $0.14 \mathrm{e}^{-3}$ ).

Table 3-6. Electron densities $\left[\mathrm{e} \AA^{-3}\right]$, Laplacians $\left[\mathrm{e}^{-5}\right]$ at the BCPs of the $\mathrm{Li}-\mathrm{O}$ and $\mathrm{Li}-\mathrm{C}$ bonds in 2.

\begin{tabular}{l|lll}
\hline & $\rho\left(\mathbf{r}_{\mathrm{BCP}}\right)$ & $\nabla^{2} \rho\left(\mathbf{r}_{\mathrm{BCP}}\right)$ & $\varepsilon\left(\mathbf{r}_{\mathrm{BCP}}\right)$ \\
\hline O1-Li1 & 0.22 & 4.68 & 0.05 \\
O1-Li2 & 0.20 & 4.44 & 0.03 \\
O1-Li2' & 0.20 & 4.30 & 0.03 \\
O1-Li3 & 0.19 & 4.08 & 0.02 \\
O1-Li5 & 0.16 & 3.04 & 0.04 \\
O1-Li7 & 0.24 & 5.66 & 0.02 \\
O1-Li9 & 0.23 & 5.34 & 0.03 \\
O3-Li7 & 0.19 & 4.38 & 0.11 \\
C1-Li1 & 0.16 & 2.57 & 0.08 \\
C1-Li2 & 0.14 & 2.22 & 0.06 \\
C1-Li3 & 0.14 & 2.27 & 0.15 \\
C2-Li1' & 0.16 & 2.57 & 0.08 \\
C2-Li2 & 0.14 & 2.22 & 0.04 \\
C2-Li5' & 0.13 & 1.96 & 0.12 \\
\hline
\end{tabular}

\subsubsection{Conclusion}

The bonding situation of $\mathrm{O}^{2-}$ captured inside an inverse crown cage has been examined by QTAIM. Since the coordination sphere of the anion is highly distorted and the distances to the surrounding lithium cations vary considerably, the coordination number cannot be deduced from an X-ray standard structure clear-cut. The existence of seven bond paths to all neighboring lithium atoms proves the existence of these attractive interactions. By evaluating the Laplacian at the BCPs, the electrostatic nature of the interactions can be confirmed. In addition, by evaluating $\rho\left(\mathrm{r}_{\mathrm{BCP}}\right)$ it can be seen that the $\mathrm{Li}-\mathrm{O}$ bonds are not equal but differ in strength. Hereby, the variances in strength are in accordance with the experimentally obtained atomic distances. 


\subsection{Hydrid}

\subsubsection{Introduction}

A further class of popular deprotonation agents are the lithium amides. ${ }^{[15]}$ Key feature of these reagents is a strong basicity in combination with a relatively low nucleophility. Therefore, in reactions involving hydrogen abstraction bulky lithium amides are often preferred over the even more basic organolithum bases since the higher reactivity of the latter can lead to more side reactions.

Without any doubt lithium diisopropyl amide (LDA) is the most prominent representative of this class. Traditionally it is used for the enolization of carbonyl compounds or esters. ${ }^{[121]}$ Nevertheless reactions involving nucleophilic addition of LDA to alkenylphosphaten, ${ }^{[122]}$ acid chlorides $^{[123]}$ benzynes $^{[124]}$ and substituted enones ${ }^{[125]}$ have been reported also. Generally, LDA is prepared in situ from $n \mathrm{BuLi}$ in hydrocarbons without further purification. Since LDA has a tendency to precipitate from hydrocarbon solution, donor ligands like thf are employed for solvatation. The resulting LDA-thf complex has been shown to be dimeric by NMR and Xray experiments. ${ }^{[126]}$

The structure principles of lithium dialky amides differ from that of lithium alkoxides presented in the last chapter. The geometrical properties of lithium alkoxides resemble that of lithium imines. Here, a face to face stacking of dimeric ot tryciclic $(\mathrm{NLi})_{\mathrm{X}}$ or $(\mathrm{OLi})_{\mathrm{X}}$ rings occurs. However, this arrangement is not possible for lithium dialkyl amides for steric reason. While in imides or alkoxides the $\alpha$-carbon atom shares a plane with the $(\mathrm{NLi})_{x}$ ring, in dialkyl amides there are two organic substituents pointing above and below the $(\mathrm{NLi})_{x}$ ring plane. This makes a face to face stacking impossible. Instead, a vertical edge to edge stacking occurs, resulting in a ring laddering (figure 3-14). ${ }^{[14]}$ 
a)

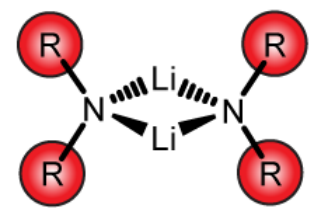

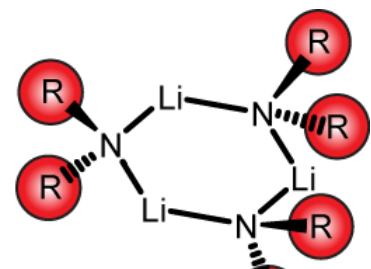

$\mathrm{R}$

b)

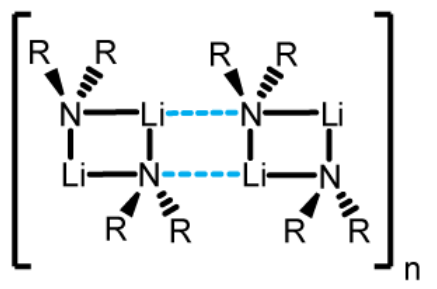

Figure 3-14. a) Bulky substituents above and below the $(\mathrm{NLi})_{n}$ plane prevent face to face stacking; $\left.\mathrm{b}\right)$ lateral association of $(\mathrm{NLi})_{\mathrm{n}}$ units," ring laddering".

For uncomplexed lithium amides, the ring laddering can extend in principle to infinite lengths leading to insoluble polymers. The ladder structures can be broken down to smaller units by the addition of donor ligands, which terminate the ladder at both ends (figure 315). ${ }^{[127]}$

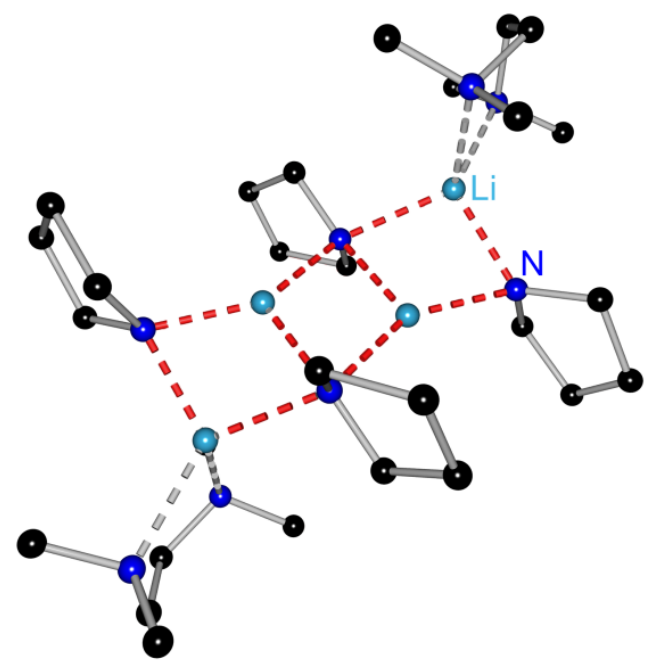

Figure 3-15. Ring ladder motif in the molecular structure of $\left\{\left[\mathrm{H}_{2} \mathrm{C}\left(\mathrm{CH}_{2}\right)_{3} \mathrm{NLi}\right] \cdot T M E D A\right\}_{2}{ }^{[127]}$

Mulvey et al. encountered a surprising aggregation behavior of LDA when he used the common chelat ligand tmeda for activation. He obtained a crystalline modification that contained no coordinating donor base at all. ${ }^{[128]}$ This is surprising because in general tmeda is considered to be a good ligand for lithium, ${ }^{[129,130]}$ generating discrete monomeric or dimeric aggregates. In hydrocarbon solution the tmeda complexed LDA has been reported to exist exclusively as a dissolvated dimer. ${ }^{[131]}$ Moreover, in this particular case LDA does not aggregate according to the ring laddering principle as anticipated for uncomplexed lithium 
amides. Instead, LDA forms a polymer by adopting an intriguing helical arrangement (figure 3-16). Every lithium atom possesses two nitrogen contacts and the resulting nearly linear $\mathrm{N}-$ $\mathrm{Li}-\mathrm{N}$ units connect to form a chain. The possibility of ring closure and subsequent ring stacking is prohibited by the tetrahedral $\mathrm{sp}^{3}$ geometry at the nitrogen atoms. Instead of that the growing chain makes a twist after four $\mathrm{N}-\mathrm{Li}-\mathrm{N}$ units and coils up to a helical polymer. The fact that this helix is not disrupted at some stage by the strong donor ligand tmeda like in the ladder structure shown in figure 3-15 is astonishing. However, traces of tmeda could be detected by NMR spectroscopy of the washed and dried crystalline material. The empirical composition was found to be $\left[\left(\mathrm{Pri}_{2} \mathrm{NLi}\right)\left(\mathrm{Pri}_{2} \mathrm{NH}\right)_{0.1}(\mathrm{tmeda})_{0.025}\right]$ by evaluation of the integrals of the ${ }^{1} \mathrm{H}$-spectrum. ${ }^{[128]}$ It is tempting to postulate that these catalytic amounts of tmeda act rather like a structure promoter helping in the building process of the helix than as a complexation reagent as usual.

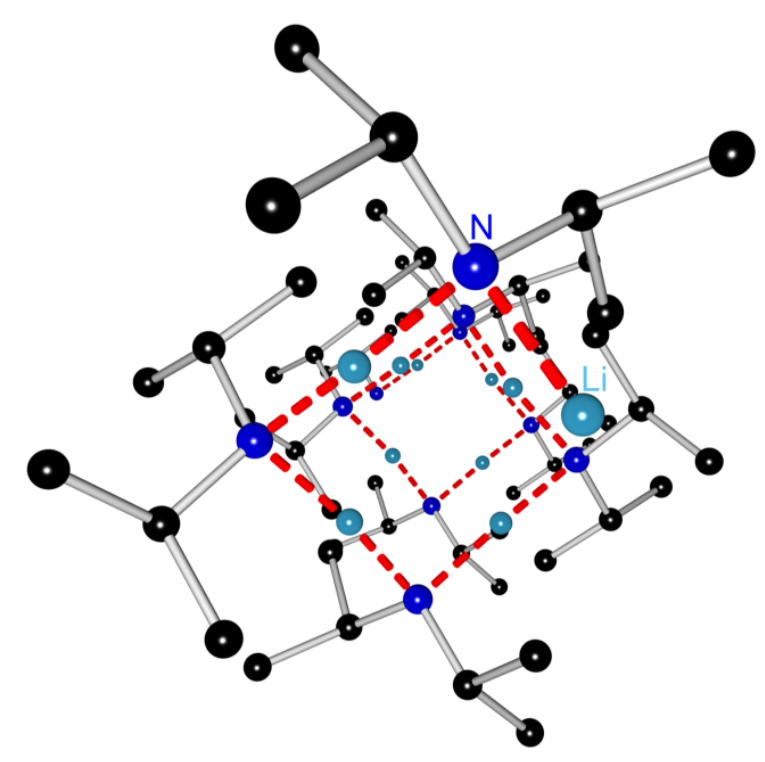

Figure 3-16. View along the helical axis of crystalline LDA. ${ }^{[128]}$

Besides the elegant way of self-assembly organometallic helices possess a property that could make them valuable for synthetic chemists: They exhibit a sense of rotation and thus can occur as left handed or right handed helices. If the feature of helical chirality could be used for asymmetric deprotonation of organic substrates, no stochiometric amount of a chiral additive would be necessary. However, owing to the fact that enantiomers have the same enthalpy of formation the ratio between left hand and right hand LDA helices is 1:1. If tmeda actual acts as a structure promoter for the helix, replacing it by an appropriate chiral diamine base could lead to the preferential construction of chiral helices.

During the studies of the building principles of such helical arrangements, which are very rare in lithium organic chemistry (as an example for a helical modification of methyllithium see Stalke et al. ${ }^{[132]}$ ), a new related inverse crown ether modification of LDA was found. In 
contrast to the majority of inverse crown ethers, which are composed of two different s-block metals, ${ }^{[111]}$ a monometallic inverse crown is being built in this case.

\subsubsection{Preparation}

Diisopropylamine was deprotonated with $n \mathrm{BuLi}$ in hexane at $0^{\circ} \mathrm{C}$. After storing the flask at $-18^{\circ}$ over night, a white solid of polymeric LDA precipitated. The LDA was washed with cold hexane and the solvent was removed in vacuo. Afterwards, the LDA precipitate was dissolved in pure tmeda at $0^{\circ} \mathrm{C}$ and placed in a freezer at $2^{\circ} \mathrm{C}$. Within a week, a separated red phase formed. After a second week, dark red crystals that were suitable for an X-Ray analysis were obtained. These consisted of the solvent separated ion pair (SSIP) $\left[\left\{(\operatorname{Pr})_{2} \mathrm{NLi}\right\}_{5} \mathrm{H}\right]^{-}\left[(\text {tmeda })_{2} \mathrm{Li}\right]^{+}$, which crystallizes in the monoclinic space group Pn. The LDA units form a five-membered $(\mathrm{NLi})_{5}$ ring in which a hydrid anion is encapsulated. The negative charge is balanced by a lithium cation which is tetracoordinated by two tmeda ligands. The origin of this hydridic guest could not be verified, but it could result from a $\beta-\mathrm{H}$ abstraction of one LDA molecule. The eliminated lithium hydrid is then solvated by an excess of tmeda and LDA (scheme 3-9).

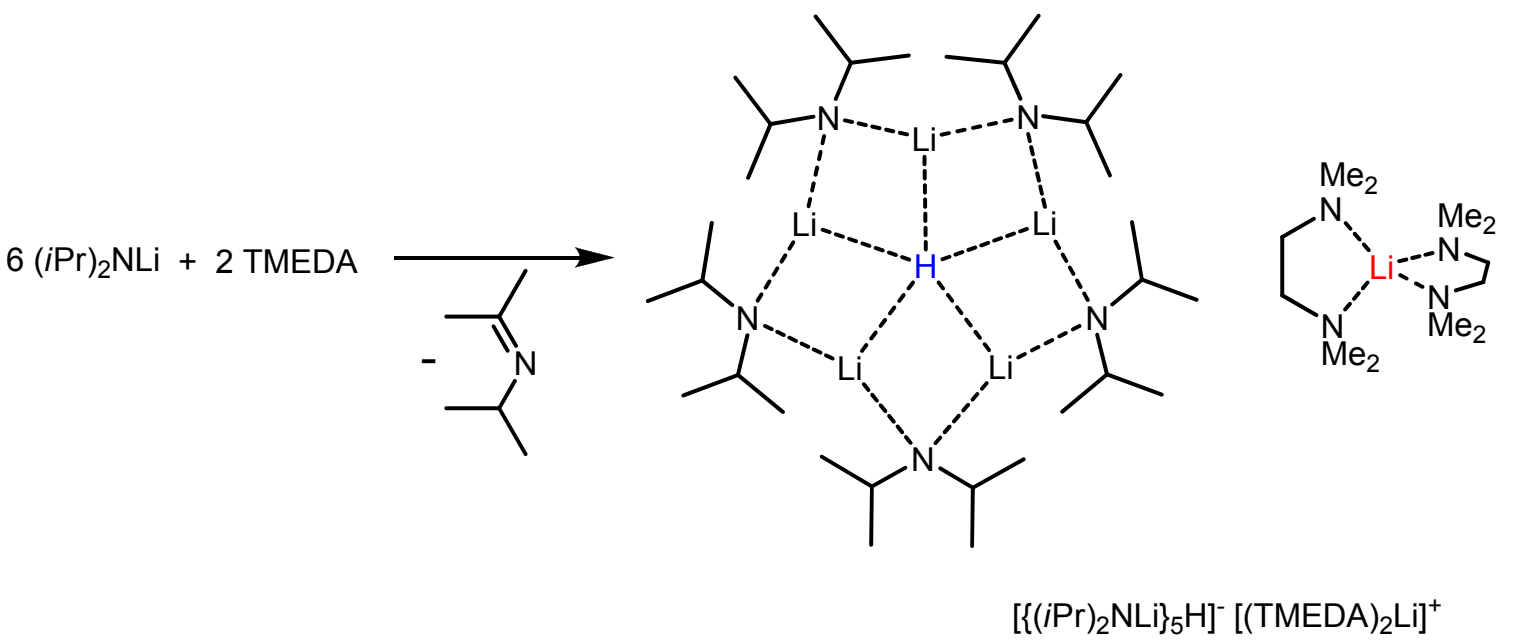

Scheme 3-9. $\beta$-H elimination of LDA.

The hydrid is solvated inside an inverse crown ether of the anionic part of 3 (figure 3-17). Every nitrogen atom in the inverse crown possesses two lithium contacts. The lithium atoms are arranged in a distorted pentagon and coordinate to the central hydrid anion. 


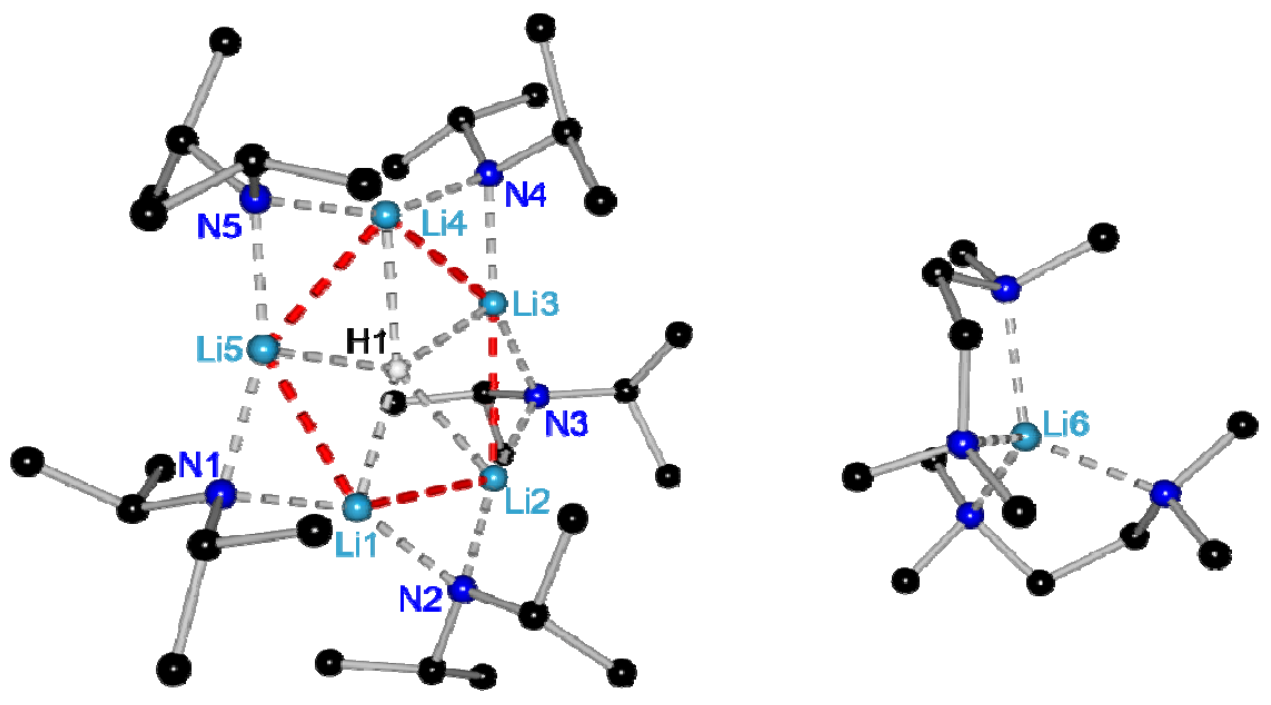

Figure 3-17. Molecular structure of Hydrid (3). 
At the beginning of the aggregation process, the structure of the LDA chain is quite similar to Mulvey's LDA-helix, but in this case, the anionic guest makes the difference. It prevents the formation of a helix by keeping all lithium atoms within a plane and the ring closes after five units. The nitrogen-lithium distances in the inverse crown ether vary between 193 and 202 pm (av. 198.7 pm). This discrepancy is notably bigger than in Mulvey's LDA helix, where each lithium is engaged in one short bond (av. 193.7 pm) and one long bond (av.195.7 pm). However, in 3 there is no alternating pattern of short and long bond distances as in the helical form. Furthermore, the mean $\mathrm{Li}-\mathrm{N}-\mathrm{Li}$ bond angle of $81.9^{\circ}$ is remarkably smaller than in Mulvey's helix (av. $108.9^{\circ}$ ). This reduction is probably due to the fact that this motif is part of a NLiLiH four-membered ring, in which the bridging lithium atoms are attracted to the hydrid anion. This effect is even stronger observable in the LDA-thf complex, where the hydrid position has been replaced by a more electronegative nitrogen atom. Therefore, the resulting $\mathrm{Li}-\mathrm{N}-\mathrm{Li}$ bond angle drops to $72.2^{\circ}$ (figure 3-18). Accordingly different angles can also be observed along the $\mathrm{N}-\mathrm{Li}-\mathrm{N}$ units. While these are essentially linear in Mulvey's helix (av. $\left.176^{\circ}\right)$, they are slightly bent in $3\left(\right.$ av. $\left.155.41^{\circ}\right)$.
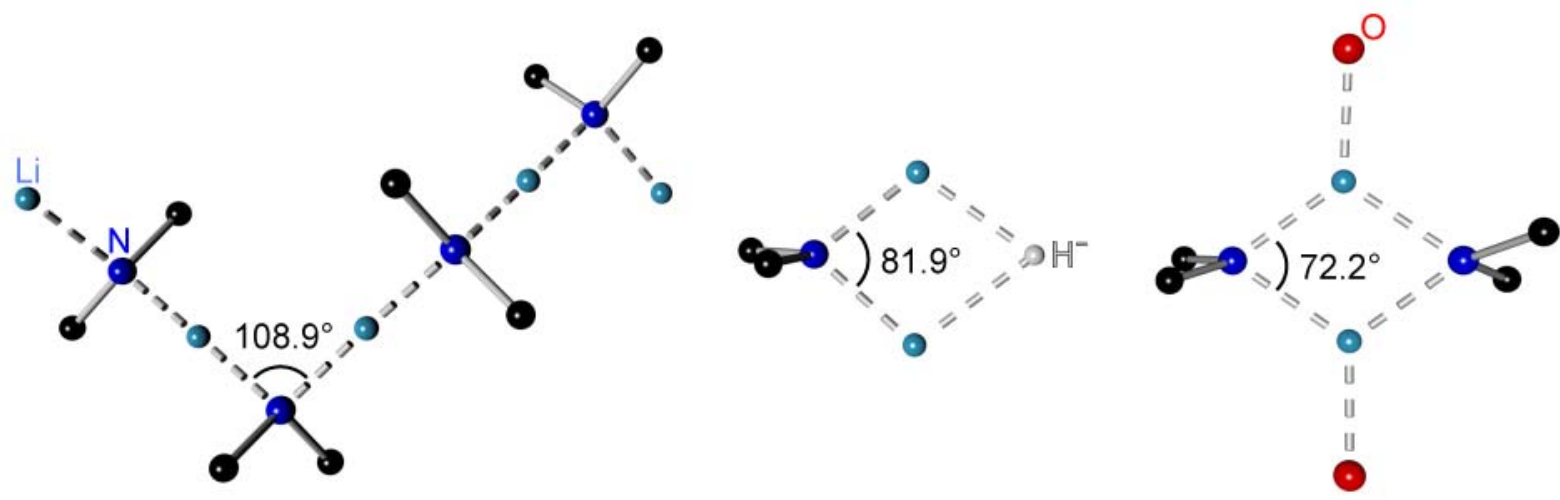

Figure 3-18. Comparison of Li-N-Li angles in Mulvey's LDA helix (left), Hydrid (3) (middle), and the dimeric LDA-thf complex (right). For clarity only structural informative parts are depicted.

The precise location of hydrogen atoms in X-ray experiments is a difficult task since the lightest atom possesses only one electron. Furthermore, due to the small electronegativity the electron density of hydrogen is strongly polarized towards the elements it is bonded too. Thus the position of the maximum of the ED does not coincidence with its atomic position. Therefore hydrogen atoms from X-ray experiments are inherently too short. In practice this problem is solved by the application of rigid body constraints. ${ }^{[133]}$ 
Table 3-7: Selected bond lengths [pm] and angles $\left[^{\circ}\right]$ for Hydrid (3) and related compounds.

\begin{tabular}{|c|c|c|c|c|}
\hline & Hydrid (3) & Mulvey's Helix ${ }^{[128]}$ & & Hydrid (3) \\
\hline Li1-N1 & $196.8(5)$ & $195.7(5)$ & $\mathrm{Li} 1-\mathrm{H} 1$ & $222(3)$ \\
\hline Li1-N2 & $201.7(5)$ & $193.4(5)$ & Li2-H1 & $249.7(13)$ \\
\hline Li2-N2 & $193.0(7)$ & 195.6(4) & Li3-H1 & 202(3) \\
\hline Li2-N3 & $194.0(7)$ & $193.9(4)$ & Li4-H1 & $208(2)$ \\
\hline Li3-N3 & $200.4(6)$ & & Li5-H1 & $220(2)$ \\
\hline Li3-N4 & 197.0(6) & & & \\
\hline Li4-N4 & $200.5(5)$ & & & \\
\hline Li4-N5 & $199.5(5)$ & & & \\
\hline Li5-N1 & $200.0(5)$ & & & \\
\hline Li5-N5 & $198.8(5)$ & & & \\
\hline Li1-N1-Li5 & $80.3(2)$ & $108.3(2)$ & & \\
\hline Li2-N2-Li1 & $83.94(19)$ & $109.5(2)$ & & \\
\hline Li2-N3-Li3 & $83.70(19)$ & & & \\
\hline Li3-N4-Li4 & $81.3(2)$ & & & \\
\hline Li5-N5-Li4 & $80.42(8)$ & & & \\
\hline N1-Li1-N2 & $150.1(3)$ & $177.9(3)$ & & \\
\hline N2-Li2-N3 & $167.15(13)$ & $174.2(2)$ & & \\
\hline N4-Li3-N3 & 149.6(3) & & & \\
\hline N5-Li4-N4 & $155.8(3)$ & & & \\
\hline N5-Li5-N1 & $154.4(3)$ & & & \\
\hline
\end{tabular}

The location of a hydrid anion is still problematic. Despite the presence of two electrons, which raises the X-ray scattering power of the element, the electronegativity of hydrogen is still too low so the additional electron density is not located at the core but smeared out over an extensive volume. The hydrid atom in $\mathbf{3}$ was found in a difference Fourier map and was refined without any constraints. It is located within a bend plane spanned by the lithium atoms Li1-Li5 (figure 3-19). Because of this deviation from a regular pentagon, the hydrid is not exactly centered and the hydrid-lithium distances vary over a broad range from 202 to $250 \mathrm{pm}$. 

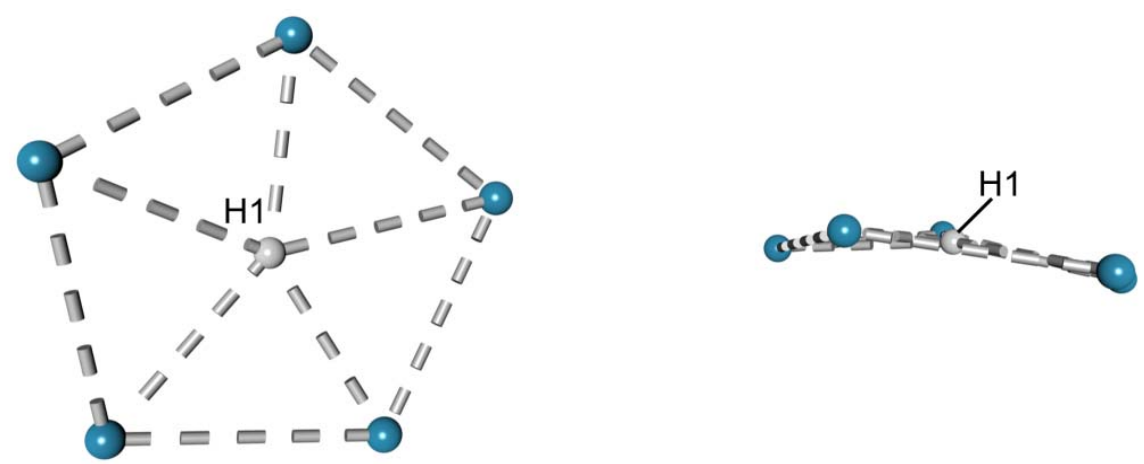

Figure 3-19. Two views on the pentagonal geometry of the lithium atoms. On the right side the deviation from a planar pentagon can be seen.

\subsubsection{Conclusion}

The molecular structure of a novel pentagonal inverse crown ether with a hydridic guest has been elucidated. This structural motif bears similarities to the helical modification of LDA and to inverse crown ethers, although these are composed of two different alkali/ earth-alkali metals in most cases. The origin of the hydrid anion could not be determined, but a $\beta-\mathrm{H}$ abstraction of one LDA molecule seems to be reasonable. 


\subsection{Simpkin's Amide}

\subsubsection{Introduction}

Since lithium amide bases posses a central position in deprotonation reactions, chiral variants of LDA for asymmetric deprotonation have been developed also. ${ }^{[29]}$ Figure 3-20 shows some representatives for homochiral lithium amide bases, which have been found to be successfully in asymmetric proton abstractions. ${ }^{[134]}$ Since these expensive reagents have to be employed usually in stochiometric amounts, strategies have been developed for the regeneration of the chiral base by using bulky lithium amide bases with a low kinetic basicity. ${ }^{[135]}$<smiles>C[C@H](c1cccc2ccccc12)N(C)[AlH]</smiles><smiles>[CH]N(Cl)[C@H](C)c1ccccc1</smiles><smiles>C[C@H](c1ccccc1)N(Cl)Cc1ccccc1</smiles><smiles>CN[C@H]1CCCC[C@H]1NCl</smiles>

Figure 3-20. Examples for chiral lithium amide bases.

One of the most employed chiral lithium amide is the $\mathrm{C}_{2}$-symmetric Simpkin's amide. Among other things it has been used for the reaction with prochiral cyclic ketones. (scheme 3-10). ${ }^{[136]}$ After quenching with $\mathrm{Me}_{3} \mathrm{SiCl}$ in situ the O-silylated product is obtained with an enantiomeric excess (ee) of $82 \%$.
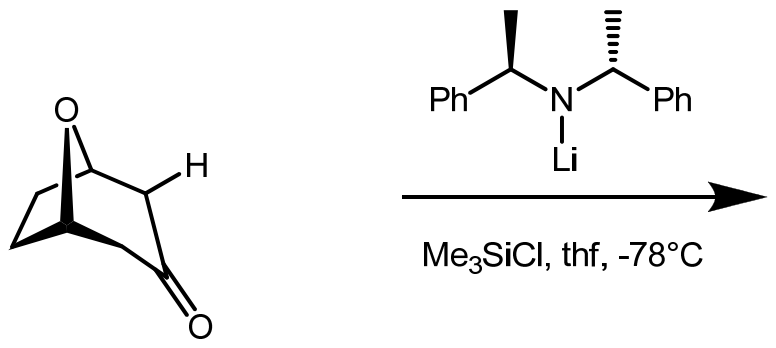

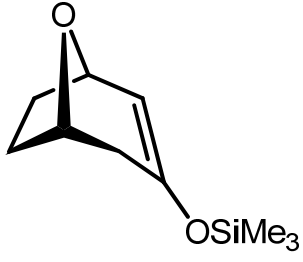

$82 \%$ ee

Scheme 3-10. Asymmetric deprotonation of a cyclic ketone by $(R, R)-\left\{[\mathrm{Ph}(\mathrm{Me}) \mathrm{CH}]_{2} \mathrm{NLi}\right\}$.

Majewski and Gleave searched for the reason of the asymmetric deprotonation and the different reactivates observed for a whole series of chiral amide bases. In many cases, the stereoselectivity of enolate formation can be explained by the Ireland model, ${ }^{[137]}$ which proceeds on the assumption that the transition state consists of a pericyclic ring. As the model failed in the case of Simpkin's amide, Majewski and Gleave evaluated the possibility 
that the active species could be dimeric. To establish the correct conformation of the dimer, they performed molecular mechanics (MM2) calculations in the gas phase. ${ }^{[138]}$ Afterwards, a transition state model including the lowest energy conformer approaching the ketone has been proposed. While their assumption on the aggregation grade could be confirmed by $X-$ ray crystal structure analysis, their postulated conformation seems to be wrong. ${ }^{[139]}$ This is probably due to the inability of the MM2 force field to describe attractive interaction between the lithium and the methyl groups of the Simpkin's amide correctly.

The crystal structure of $(R, R)-\left\{[\mathrm{Ph}(\mathrm{Me}) \mathrm{CH}]_{2}-\mathrm{NLi} \cdot \mathrm{thf}\right\}_{2}$ is similar to the structure of LDA-thf featuring the known four-membered $\left(\mathrm{N}_{2} \mathrm{Li}_{2}\right)$-ring motif (figure 3-21, left). The plane containing the amidic nitrogens and $\mathrm{C} 1$ and $\mathrm{C} 2$ is almost orthogonal to the $\left(\mathrm{N}_{2} \mathrm{Li}_{2}\right)$ ring plane. Yet the most eye-catching fact is the orientation of the four methyl groups of the chiral amide side chains. They feature remarkably short carbon-lithium distances, C3 and C4 being located closest to the lithium atoms (C4-Li1 274.1(14) pm and C3-Li2 278(2) pm). This causes the thf ligands to be pushed above the $\left(\mathrm{N}_{2} \mathrm{Li}_{2}\right)$ ring into a cisoid arrangement. Since these methyl-lithium interactions are neglected in the MM2 calculations, which leads to a wrong conformation, the molecular structure from the X-ray experiment appears to be a better model for the rationalization of the enantioselectivity.
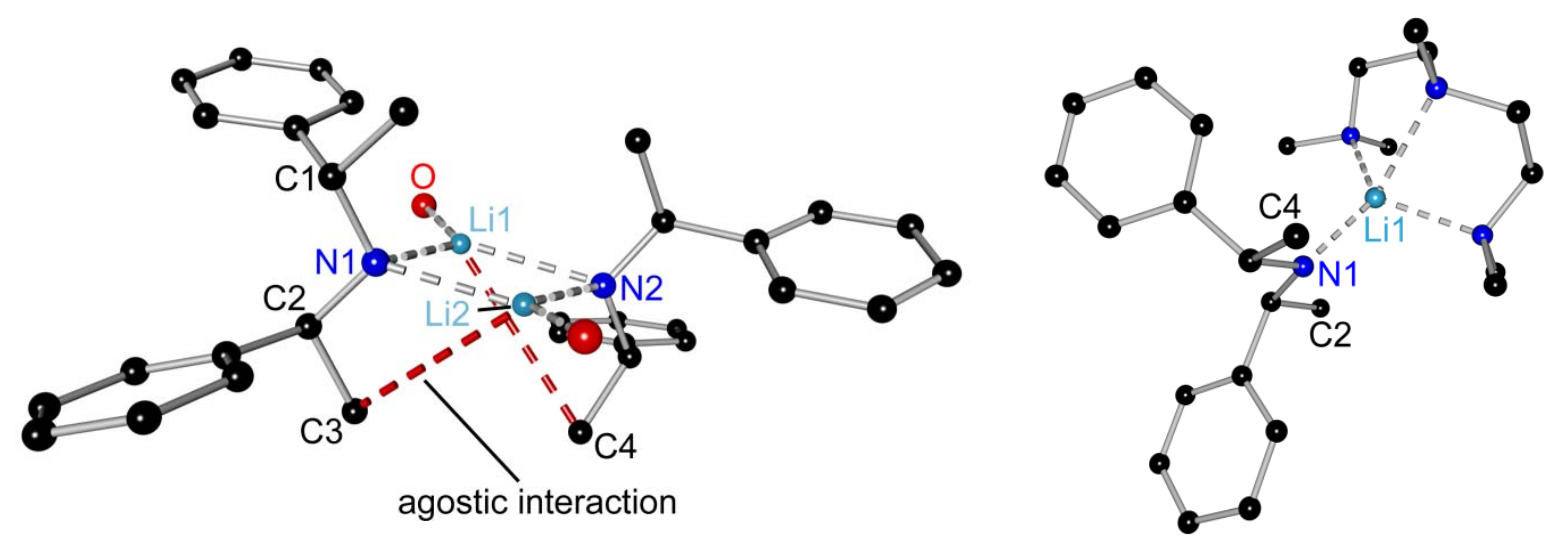

Figure 3-21. Molecular structures of $(R, R)-\left\{[\mathrm{Ph}(\mathrm{Me}) \mathrm{CH}]_{2}-\mathrm{NLi} \cdot \mathrm{thf}_{2}{ }^{[139]}\right.$ (left, carbon atoms of thf omitted for clarity) and $(R, R)-\left\{[\mathrm{Ph}(\mathrm{Me}) \mathrm{CH}]_{2} \mathrm{NLi} \cdot \mathrm{pmdeta}\right\}^{[140]}$ (right).

To generate the monomer, Andrews et al. applied the tridentate donor base pmdeta. ${ }^{[40]}$ This results in a tetrahedral coordination of the lithium atom with one lithium-amide contact and three $\mathrm{N}-\mathrm{Li}$ donor bonds (figure 3-21, right). The positions of the methy groups are of special interest here also. As in $(R, R)-\left\{[\mathrm{Ph}(\mathrm{Me}) \mathrm{CH}]_{2}-\mathrm{NLi} \cdot \operatorname{thf}\right\}_{2}$, an asymmetry for the methyllithium distances can be found. However, the analog carbon-lithium distances that have been assumed to have some influence on the stereochemical control ${ }^{[139]}$ are clearly longer in $(R, R)-\left\{[\mathrm{Ph}(\mathrm{Me}) \mathrm{CH}]_{2} \mathrm{NLi} \cdot \mathrm{pmdeta}\right\}$ (289.9 and $\left.332.5 \mathrm{pm}\right)$. 
Thus, with thf as a single donor and pmdeta as a tridentate ligand the dimer and the monomer of Simpkins's amide have been synthesized. The missing peace between is the didentate donor tmeda, that already showed an unpredictable behavior in the presence of parent LDA. Since the molecular structure of LDA-thf consists with the thf-solvate of the chiral amide, it is interesting to see whether it is also possible in the case of tmeda to transfer the knowledge of basic lithium structures to slightly altered ones. The question arises, whether the Simpkin's amide would be able to forms a respective chiral helix, maybe even supported by a m-interaction of the phenyl rings, or does a hydrogen abstraction occur resulting in the formation of a chiral inverse crown ether?

\subsubsection{Preparation}

The synthesis of $(R, R)-\left\{[\mathrm{Ph}(\mathrm{Me}) \mathrm{CH}]_{2} \mathrm{NLi} \cdot\right.$ tmeda $\}$ is analog to the preparation of 3 (scheme 3-11). After one day of storage at $-18^{\circ} \mathrm{C}$, the solution was filtrated and stored for a further week at $-18^{\circ} \mathrm{C}$. Dark red crystals that were suitable for X-ray analysis could be obtained.
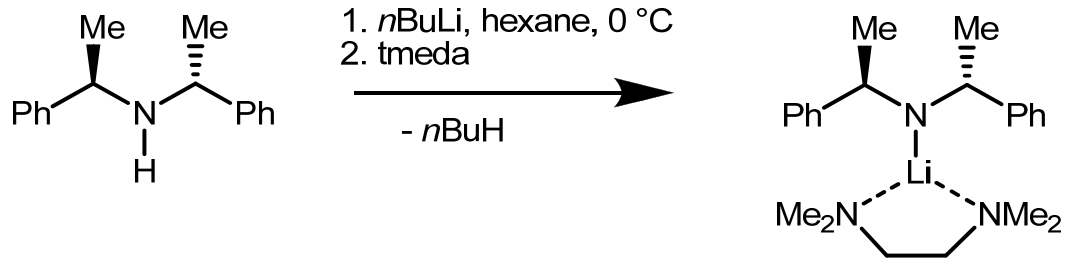

Scheme 3-11. Synthesis of $(R, R)-\left\{[\mathrm{Ph}(\mathrm{Me}) \mathrm{CH}]_{2} \mathrm{NLi} \cdot \mathrm{tmeda}\right\}$ (4).

4 crystallizes in the chiral space group $P 2{ }_{1} P 2_{1} P 2_{1}$ (figure 3-22). The asymmetric unit contains half of the molecule. The complete molecule is generated by a $\mathrm{C}_{2}$-axis going to the N1-Li1 trajectory. Opposite to the situation in LDA, this time tmeda is successful in deagreggating the Simpkin's amide to a discrete monomer. Obviously, the substitution of two methyl groups by sterically more demanding phenyl groups is sufficient to suppress a vertical stacking or the formation of a helical arrangement. 


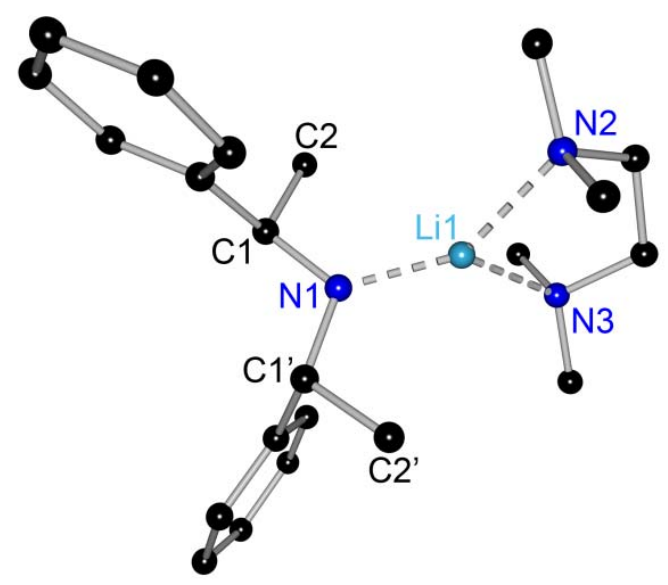

Figure 3-22. Molecular structure of 4.

The lithium atom is only tricoordinate. This coordination number of lithium in monomers is much less frequently and occurs typically either in the presence of bulky bases like sparteine ${ }^{[32]}$ or when sterically demanding substituents of the organic residue prevent the accretion of further ligands.

The amide-lithium bond (186.3(4) pm) is only by $10 \mathrm{pm}$ shorter than the two $\mathrm{N}-\mathrm{Li}$ donor bonds $(206.7(3) \mathrm{pm})$. In the comparable $(R, R)-\left\{[\mathrm{Ph}(\mathrm{Me}) \mathrm{CH}]_{2} \mathrm{NLi} \cdot\right.$ pmdeta $\}$ these bond lengths differ considerably. While the N1-Li1 contact is slightly longer than in 4 (194.9(6) pm), the lithium-nitrogen distances to the pmdeta ligand are for steric reasons substantially elongated (av. $222.4 \mathrm{pm}$ ). They are also notably longer than the corresponding $\mathrm{N}-\mathrm{Li}$ donor bonds in 2AnisolLi-pmdeta (1), which have a mean value of $214.5 \mathrm{pm}$. Of special interest are the distances $\mathrm{C} 2 / \mathrm{C} 2$ ' to lithium. While in the pmdeta and the thf analog there are two sets of long and short carbon-lithium contacts, because of crystal symmetry these distances are identical in 4. In addition, these are remarkably longer (302.2 pm) than the short distances in the thf (av. $276.1 \mathrm{pm}$ ) and the pmdeta derivative $(289.9 \mathrm{pm}$ ). Although tmeda is sterically less demanding than pmdeta, allowing the amidic nitrogen to approach closer to lithium (186.4(4) vs. 194.9(6) pm), this is not accompanied by a decrease in the C2/C2'-Li1 bond lengths. Although lithium is only tricoordinate a stabilizing interaction of this kind does not seem to occur. Furthermore, it has to be noted that the orientation of tmeda does not prevent such an interaction. $\mathrm{C} 2$ and $\mathrm{C} 2$ ' deviate from the plane defined by $\mathrm{C} 1, \mathrm{~N} 1$ and $\mathrm{C} 2$ by $16.82^{\circ}$. The angle between this plane and the N2-Li1-N3 is close to orthogonal $\left(67.5^{\circ}\right.$ ) (figure $3-23$ ). Thus the orientations of the $\mathrm{NMe}_{2}$ groups do not prevent $\mathrm{C} 2 / \mathrm{C} 2$ ' to establish an agostic interaction to the lithium cation. 


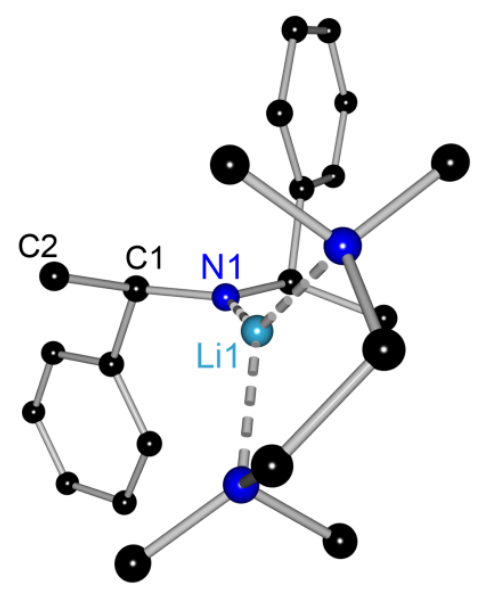

Figure 3-23. View along the $\mathrm{N}-\mathrm{Li}$ axis showing the tilt of the tmeda ligand.

Table 3-8. Selected bond lengths $[\mathrm{pm}]$ and angles $\left[^{\circ}\right]$ for $(R, R)-\left\{[\mathrm{Ph}(\mathrm{Me}) \mathrm{CH}]_{2} \mathrm{NLi} \cdot \mathrm{tmeda}\right\}(4)$ and related compounds.

\begin{tabular}{r|ccl}
\hline \multicolumn{2}{c}{$\begin{array}{c}(R, R)- \\
\left\{[\mathrm{Ph}(\mathrm{Me}) \mathrm{CH}]_{2} \mathrm{NLi} \cdot \mathrm{tmeda}\right\} \\
(\mathbf{4})\end{array}$} & $\begin{array}{c}(R, R)- \\
\left\{[\mathrm{Ph}(\mathrm{Me}) \mathrm{CH}]_{2} \mathrm{NLi} \text {.pmdeta }{ }^{[140]}\right.\end{array}$ & $\begin{array}{c}(R, R)-\left\{[\mathrm{Ph}(\mathrm{Me}) \mathrm{CH}]_{2}-\right. \\
\mathrm{NLi} \cdot \operatorname{thf}\}_{2}^{[139]}\end{array}$ \\
\hline N1-Li1 & $186.3(4)$ & $194.9(6)$ & av. 204.1(14) \\
N2-Li1 & $206.7(3)$ & $234.3(6)$ & - \\
N3-Li1 & $206.7(3)$ & $222.2(6)$ & - \\
N4-Li1 & - & $217.0(5)$ & - \\
C2-Li1 & 302.2 & 289.9 & av. 276.1 \\
\hline
\end{tabular}

\subsubsection{Conclusion}

The search for catalytic deprotonation systems still is a rewarding task. Most techniques employed today require ether stochiometric amounts of a chiral additive or stochiometric usage of a chiral lithium amide base.

For the development of new catalytic variants, detailed knowledge of the structurereactivity pattern of lithium amides is indispensable. The structural diversity of these compounds makes it often difficult to predict the molecular structure of new reagents even if there is already a huge amount of knowledge on parent structures available.

The origin of the enantioselectiviy of Simpkin's amide remains unclear. Mair and Simpkins emphasized that "maximization of methyl contacts to lithium" is a structure determining factor. As these attractive interaction lead to a conformation of the chiral side groups with a lower energy they, concluded that they should also play an important role for the transition state. In monomeric $(R, R)-\left\{[\mathrm{Ph}(\mathrm{Me}) \mathrm{CH}]_{2} \mathrm{NLi} \cdot \mathrm{pmdeta}\right\}$ such short carbon-lithium distances cannot be retrieved. One could argument that the reason for this is founded in the steric 
demand of pmdeta and lithium's electrondeficiency being saturated by three nitrogen donor atoms. However, in the synthesized tmeda analog, these two factors are clearly attenuated. In spite of that, the corresponding carbon-lithium distances are over 12 pm longer. Thus it has to be questioned whether carbon-lithium interactions represent a structure determining factor or even play a role for the enantioselectivity of Simpkin's amide. 


\section{ZiNCATE COMPLEXES}

\subsection{Structures of Trimethylzincate}

\subsubsection{Introduction}

Bimetallic reagents, which are composed of one alkali metal ion, a second metal atom $(\mathrm{Zn} / \mathrm{Al} / \mathrm{Mg})$ and variable ligands have gained much attention over the last few years since they show a reactivity pattern that outperforms simple monometallic species easily. ${ }^{[43]}$ Especially zincate complexes, although known to chemists for more than 150 years, ${ }^{[141]}$ recently have been discovered to metalate substituted aromatic substrates at positions, which can neither be reached by common organolithium nor by organomagnesium compounds alone.

The precursors of zincate complexes are the neutral diorganozinc reagents. Since the electronegativity of zinc is quite low compared to lithium or magnesium, the zinc-carbon bond offers a more pronounced covalent character. The resulting lower reactivity might appear as a disadvantage at first glance. However, this characteristic allows the tolerance of many functional groups which turns the need for protecting groups dispensable. Though to compensate for the low reactivity it is often necessary to activate these reagents with amino alcohols ${ }^{[142]}$ or transition metals. ${ }^{[143,144]}$ For example, polyfunctional organozinc reagents have been used successfully in Negishi cross coupling reactions (figure 4-1). ${ }^{[145]}$
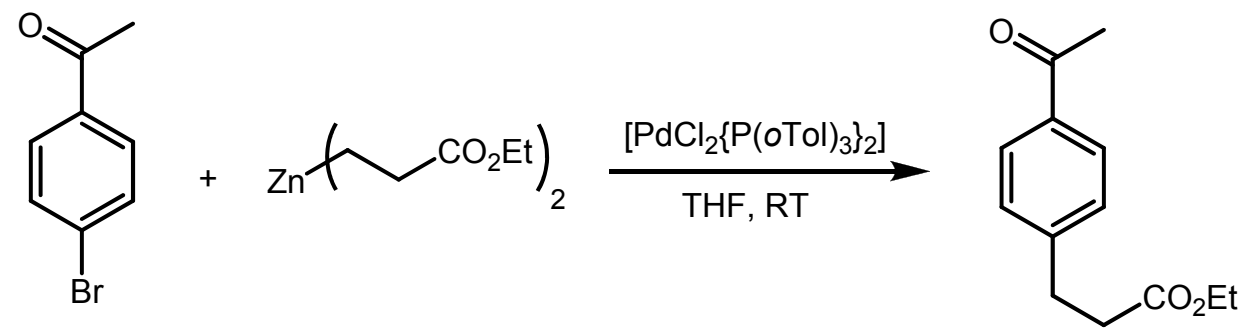

Figure 4-1. Reaction of 1-(4-bromophenyl)ethanone with a functionalized organozinc reagent in a Negishi cross coupling reaction. ${ }^{[145]}$

A drastic increase of reactivity is observed, when these organometallics form ate complexes. In diorganozinc reagents the metal atom exhibits a Lewis acidity because of a vacant orbital. Furthermore, the coordination sphere is not completed which provides the opportunity for a second nucleophilic organometall reagent - for example an organolithium to attack. This leads to the formation of ate complexes of the general formula $R_{3} Z n L i$ or $\mathrm{R}_{4} \mathrm{ZnLi}_{2}$ (scheme 4-1). Already in 1917 Schlenk and Holtz elaborated in their milestone paper on alkali-metal organics ${ }^{[146]}$ that the reaction of diethylzinc and lithium or sodium yields only zinc and an alkali-metal ethylzinc compound; hence, transmetalation does not occur. An 
unexpected transmetalation reaction of a organolithium compound and dimethyl zinc to give a diorgano zinc complex was reported as well. ${ }^{[147]}$

First investigations on the formation and reactivity of these compounds were performed by Wittig et al. in $1951 .{ }^{[148]}$ For this purpose they added ethereal solutions of phenyllithium and diphenylzinc. A first informative basis on the formation of a new adduct was supplied by a negative Gilman test. ${ }^{[149]}$ Even after exceeding the 1:1 ratio of $\mathrm{PhLi} \mathrm{Ph}_{2} \mathrm{Zn}$, the test stays negative until 1.5 equivalents of organolithium reagent have been added. $A$ further increase in PhLi concentration lastly results in a strong positive Gilman test, indicating the presence of the free organolithium reagent. These findings anticipate the formation of higher coordinated lithium zincate species. Their reactivity could be demonstrated at the deprotonation of fluorene, which lithiated form features a strong orange color. Remarkably, by this means it was possible to relate the reaction time to the discoloring speed of the solution. Mobley and Berger performed NMR studies on the system $\mathrm{Me}_{3} \mathrm{ZnLi} / \mathrm{Me}_{4} \mathrm{ZnLi}_{2}$ and were able to show that the equilibrium is on the side of the lower coordinated zincate. ${ }^{[150]}$ However, higher coordinated zincates are believed to be more reactive. In many reactions less stable $\mathrm{R}_{4} \mathrm{ZnLi}_{2}$ is assumed to be the active species which consumption during the reaction shifts the equilibrium to the right side. ${ }^{128]}$

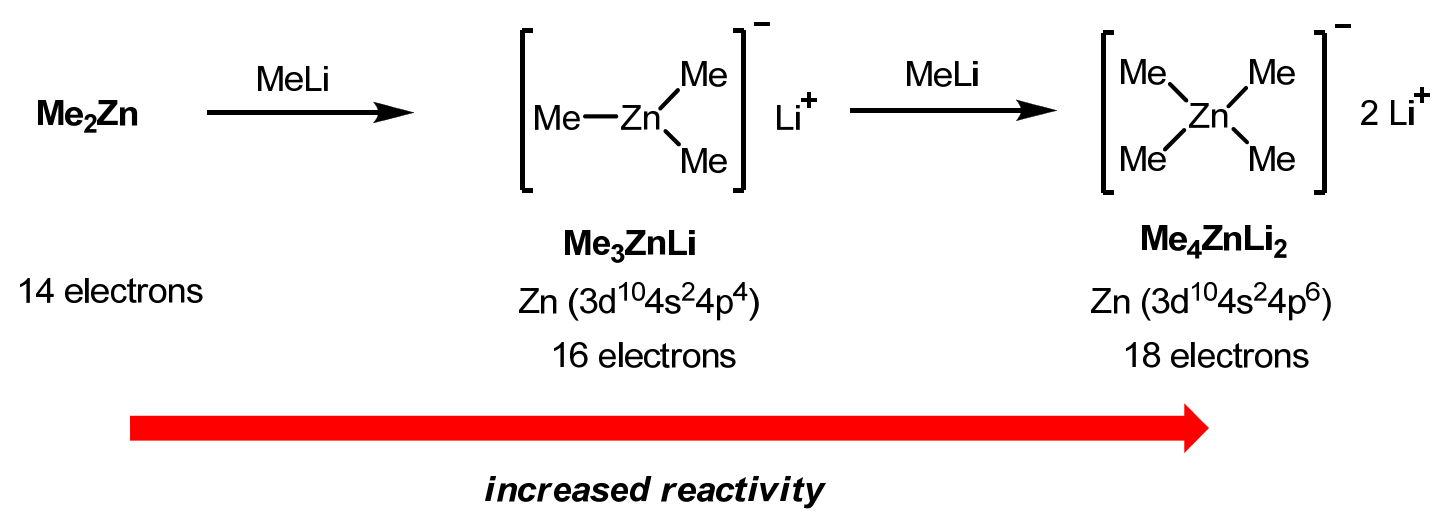

Scheme 4-1. Reactivity increase from diorganozinc reagents over triorganozincates to higher coordinated zincates.

The formation of ate species is not limited to the case that a second organometall reagent is added. In the presence of coordinating ligands diorganozinc reagents can disproportionate leading to small amounts of $\mathrm{R}_{3} \mathrm{Zn}^{-}$also. These are believed to be responsible for diethylzinc mediated deprotonations of a number of hydrocarbons. ${ }^{[151]}$

Because of the higher negative charge triorganozincates possess a substantially stronger nucleophilic power than their neutral divalent forerunners. This is reflected by an extended scope of reactions (scheme 4-2), which involves 1,4-conjugated addition with $\alpha, \beta-$ unsaturated carbonyl compounds, ${ }^{[152]}$ metal-halogen exchange reactions of aromatic 
compounds, ${ }^{[153]}$ or reduction of carbonyl groups. ${ }^{[154]}$ In the more recent past their qualification as deprotonation agents has also been documented. ${ }^{[155]}$

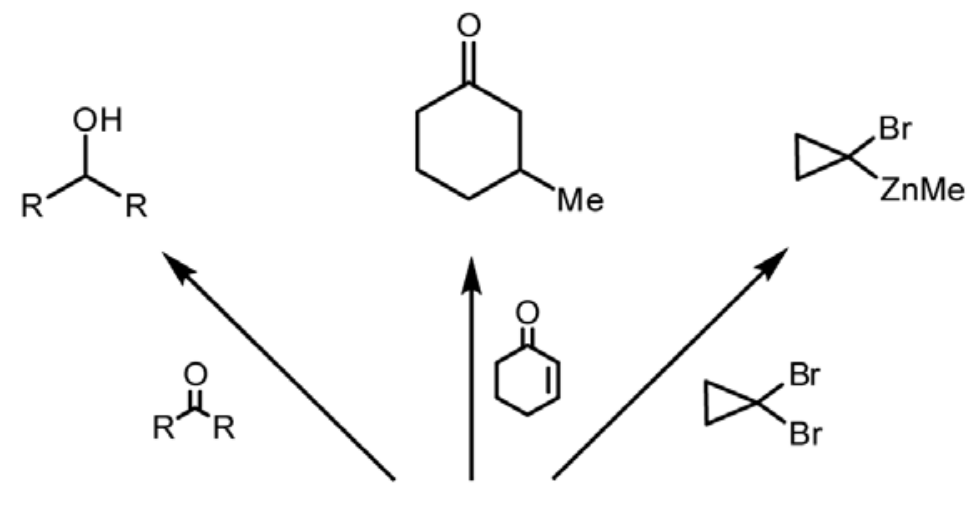

\section{$\left[\mathrm{LiZnMe}_{3}\right]$}<smiles>Ic1ccccc1</smiles>

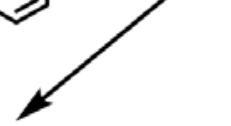<smiles>CC(C)c1ccccc1</smiles>
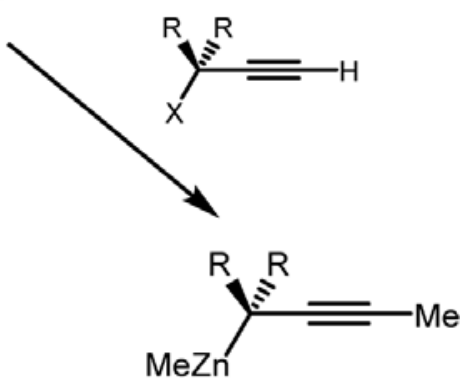

Scheme 4-2. Selected reactions of classical triorganozincates.

The first crystallographically characterized lithium zincate was solvent free dilithium tetramethylzincate $\mathrm{Me}_{4} \mathrm{ZnLi}_{2}{ }^{[156]}$ It consist of tetrahedral $\mathrm{ZnMe}_{4}{ }^{-}$units and isolated lithium counter ions (figure 4-2).

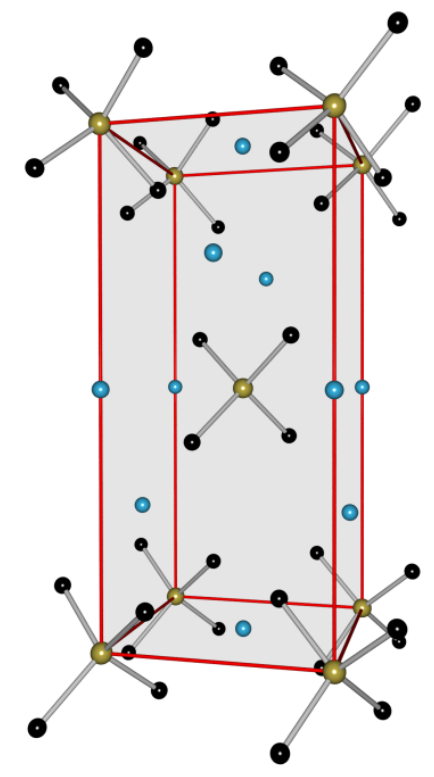

Figure 4-2. Molecular structure of solvent free $\mathrm{Me}_{4} \mathrm{ZnLi}_{2} \cdot{ }^{[156]}$ 
While triorganozincates were well-established in the field of organometallic synthesis for the last 50 years, the reactivity of higher coordinated zincates was not well studied until the 1990's. Uchiyama and coworkers prepared reagents of this type by adding methyllithium, lithium cyanide and lithium thiocyanide to thf solutions of trimethylzincate. To monitor the becoming of the new zincate species they used NMR spectroscopy. The ${ }^{1} \mathrm{H}$ resonances of the methyl groups in these organometallics is usually shifted to high field in the NMR spectrum due to the anionic charge which shields the protons from the external magnetic field. The degree of the high field shift can be approximately related to the negative charge and the nucleophilic power of the metal reagent. The ${ }^{1} \mathrm{H}$-shifts of common organometallic reagents are depicted in table 4-1.

\begin{tabular}{l|c} 
Table 4-1. ${ }^{1} \mathrm{H}-\mathrm{NMR}$ shifts $[\mathrm{ppm}]$ in thf for selected organometallic reagen \\
\cline { 2 - 2 } organometal reagent & $\delta_{\mathrm{Me}}$ \\
\hline $\mathrm{MeLi}$ & -1.96 \\
$\mathrm{MeMgBr}$ & -1.62 \\
$\mathrm{Me}_{3} \mathrm{Al}$ & -0.82 \\
$\mathrm{Me}_{2} \mathrm{Zn}$ & -0.84 \\
$\mathrm{Me}_{3} \mathrm{ZnLi}$ & -1.08 \\
$\mathrm{Me}_{4} \mathrm{ZnLi}{ }_{2}$ & -1.44 \\
\hline
\end{tabular}

Indeed the high field shift in the series $\mathrm{Me}_{2} \mathrm{Zn}>\mathrm{Me}_{3} \mathrm{ZnLi}>\mathrm{Me}_{4} \mathrm{ZnLi}$ indicates a more anionic character of the higher coordinated zincates. The methyl signal for $\mathrm{Me}_{4} \mathrm{ZnLi}$ was observed as a sharp singulett at $-1.44 \mathrm{ppm}$ between the signals of $\mathrm{MeLi}$ and $\mathrm{Me}_{3} \mathrm{ZnLi}$. Subsequently, Uchiyama et al. explored they scope of reactivity of the new organozinc derivatives toward halogen-zinc exchange, Michael additions, carbozincations and epoxide ring opening reactions. In all cases a remarkable increase of reactivity for the tetraorganozincates could be observed. They even accomplish reactions, where MeLi or $\mathrm{Me}_{3} \mathrm{ZnLi}$ show no outcome at all. For example, in the bromine-zinc exchange of bromobenzene neither organolithium nor triorganozincate reagents did afford any yield, while the reaction with $\mathrm{Me}_{4} \mathrm{ZnLi}$, proceeded to give benzhydrol in $47 \%$ yield after quenching with benzaldehyde. In 2008 the same working group published the development of the new highly chemoselective zincate base dilithium tetra-tert-butylzincate $t \mathrm{Bu}_{4} \mathrm{ZnLi}_{2}{ }^{[157]}$ Among metalhalogen exchange reactions this dianion-type zincate also promotes $S_{N} 2$ reactions with propargyl bromides. The high regioselectivity hereby has been attributed to the bulkiness of the $t \mathrm{Bu}$ groups. Furthermore, $t \mathrm{Bu}_{4} \mathrm{ZnLi} \mathrm{i}_{2}$ can serve as an initiator for the anionic polymerization of styrene and various isopropylacrylamides. 
The next big progress in organozinc chemistry was achieved with the introduction of mixed amidozincates. In 1999, Kondo and coworkers reported on the chemoselective zincation of various functionalized aromatics using a tmp zincate base. ${ }^{[52]}$ Although it has been recognized at this time that precomplexation between tmpLi and the organozinc reagent is essential for the metalation process, except for NMR studies in thf no information on this bimetallic reagent was known. In the following years the work of Knochel, Uchyiama and Kondo pushed the limits of ate chemistry to a new level making reactions possible that are unimaginable with their monometallic forerunners. The crucial aspect is the smooth metalation of many substituted aromatics where the traditionally used lithiumorganics fail because of their incompatibility with many functional groups. Scheme 4-3 summarizes some of the most important reactions involving zincate bases.

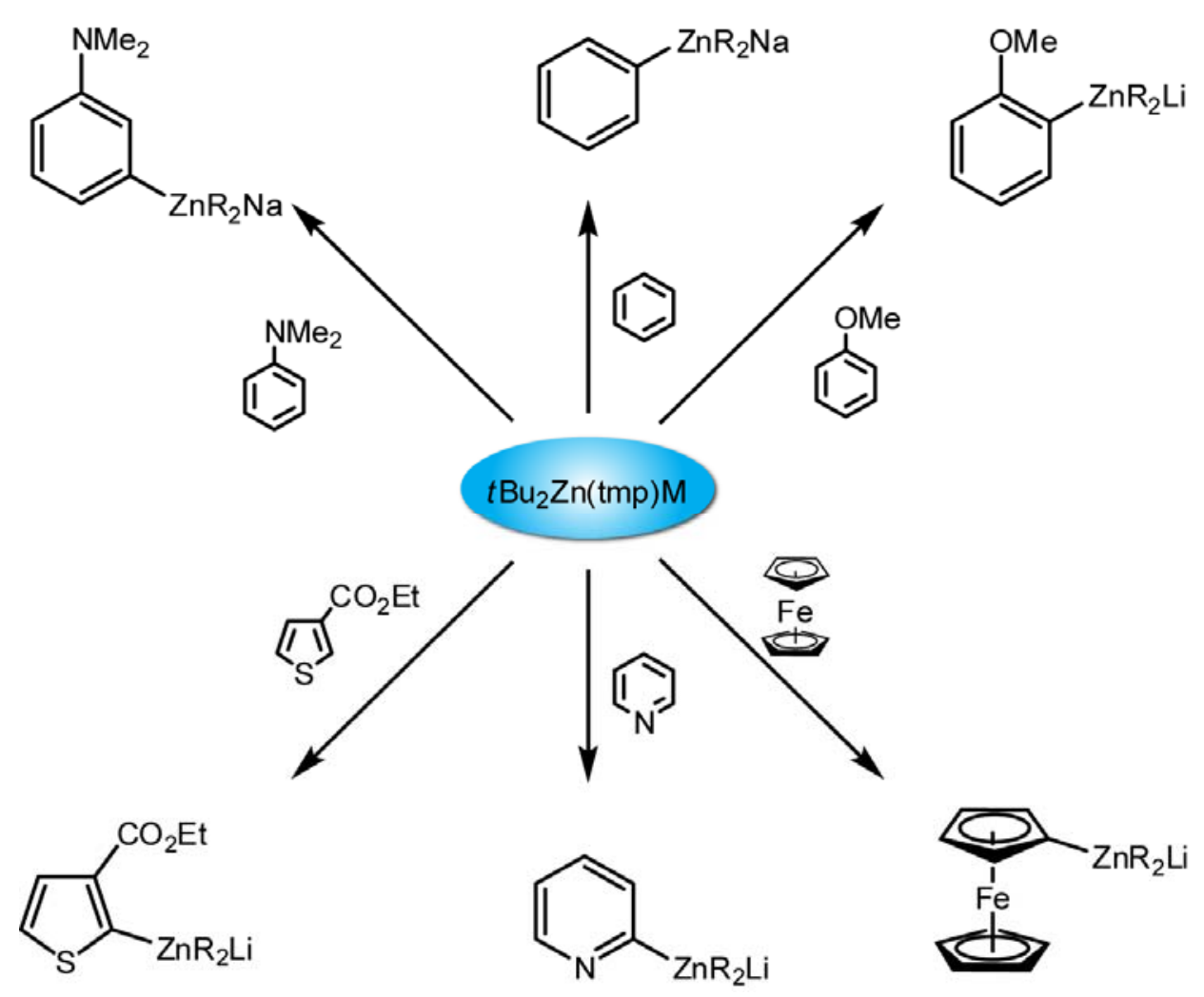

Scheme 4-3. Selected reactions of novel amido zincates.

The fast-growing number of new transformations based on ate complexes created the demand for structural information to obtain a better understanding of the synergistic effects taking place in these heterobimetallic compounds. Especially the work of Mulvey et al. contributed to the structural elucidation of many ate complexes revealing the building principles of these novel compounds. Besides the molecular structure of Kondo's original tmp zincate base, ${ }^{[158]}$ they discovered various aromatic functionalisations and were able to characterize important reactive intermediates along the reaction pathway by X-ray crystallography. An illustrative example is the deprotonation of benzene with a sodium 
zincate base. ${ }^{[159]}$ As no functional group is present in this case neither a precomplexing step nor an acidification of the aromatic protons through inductive effects can occur. For this reason benzene is relatively inert concerning metalation with organolithiums or organomagnesium compounds that have not been activated before. Likewise, the components of the mixed metal base, sodium tmp and di-tert-butylzinc, are not in the position to react with benzene on their own. However, a mixture of both leads to the formation of the ion-contacted [(tmeda) $\left.\mathrm{Na}\left(\mu-{ }^{t} \mathrm{Bu}\right)(\mu-\mathrm{tmp}) \mathrm{Zn}^{t} \mathrm{Bu}\right)$. The contact between both metals is mainly achieved by the bridging of one tmp ligand. However, short distances to the methyl group $\mathrm{C} 1$ of one $t \mathrm{Bu}$ ligand are a possible sign for a supplementary agostic interaction (scheme 4-4, left side). This frames a five-membered ( $\mathrm{NaNZnCC}$ ) ring system which rigid conformation keeps the metal centers into close proximity, which is essential for bimetallic cooperative effects to arise. These allow the smooth deprotonation of benzene at ambient temperatures.

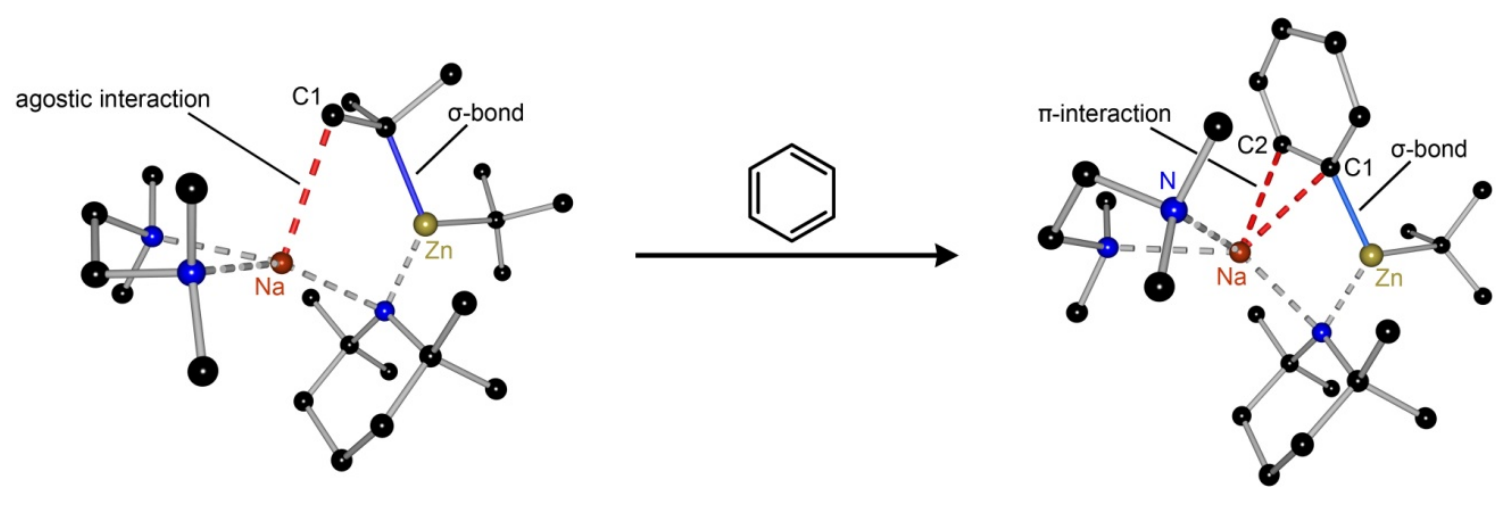

Scheme 4-4. AMMZ of benzene. ${ }^{[159]}$

The metalated product was isolated also and its molecular structure was determined (scheme 4-4, right side). At first sight the retention of the structure can be seen. The orientations of all ligands in the product concord with the educt structure except for the bridging $t \mathrm{Bu}$ group that has been replaced by the deprotonated phenyl ring. From the fact that the zinc atom is nearly in the plane of the phenyl ring, while the sodium atom is basically orthogonal above the aromatic ring plane, a $\delta$-bond between $\mathrm{Zn}$ and $\mathrm{C} 1$ and a $\pi$-type interaction between $\mathrm{C} 1 / \mathrm{C} 2$ and $\mathrm{Na}$ can be assumed. This metal-phenyl bonding type has been observed in Mg-Na inverse crown ethers before. ${ }^{[111]}$ Thus at the end a zincation of benzene takes place although in general organozinc reagents are far less reactive than organosodium bases. These findings brought Mulvey to coin the phrase 'alkali metal mediated zincation' (AMMZ) for reactions of this kind.

Regarding the metalation of substituted aromatics, important mechanistic details could be deduced from isolated reaction intermediates too. The AMMZ of $N, N$-diisopropylbenzamide 
proceeds with a high ortho selectivity which suggests a comparison to the DoM reaction discussed in a previous chapter. There especially the existence of a complex induced proximity effect (CIPE) is the matter of debate. While kinetic data of Collum et al. question the importance of the CIPE there is no structural proof for a precomlexation adduct between an organolithium and a mono substituted aromatic ring. In the reaction of $N, N-$ diisopropylbenzamide with Kondo's original base [(thf) $\mathrm{Li}(\mu-\operatorname{tmp})(\mu-t \mathrm{Bu}) \mathrm{Zn}(t \mathrm{Bu})]$ an authentic pre-metalation complex of this type could be crystallized and the structure was determined. ${ }^{[158]}$ Again a striking compliance between educt and product structures, in this case metalation agent and the formed precomplex, can be observed (scheme 4-5, left side). Although sodium and tmeda have been replaced by lithium and one thf in comparison to the ate base of the last example the subjacent structural motif remains unchanged. The agostic interaction in the lithium zincate $(241.0(6) \mathrm{pm})$ is even shorter than in the sodium analog $(275.0(10) \mathrm{pm})$. In the pre-matalation complex thf has been repelled by $\mathrm{N}, \mathrm{N}$ diisopropylbenzamide which coordinates to lithium via $\mathrm{O} 1$ of its functional group (scheme 45 , right side).
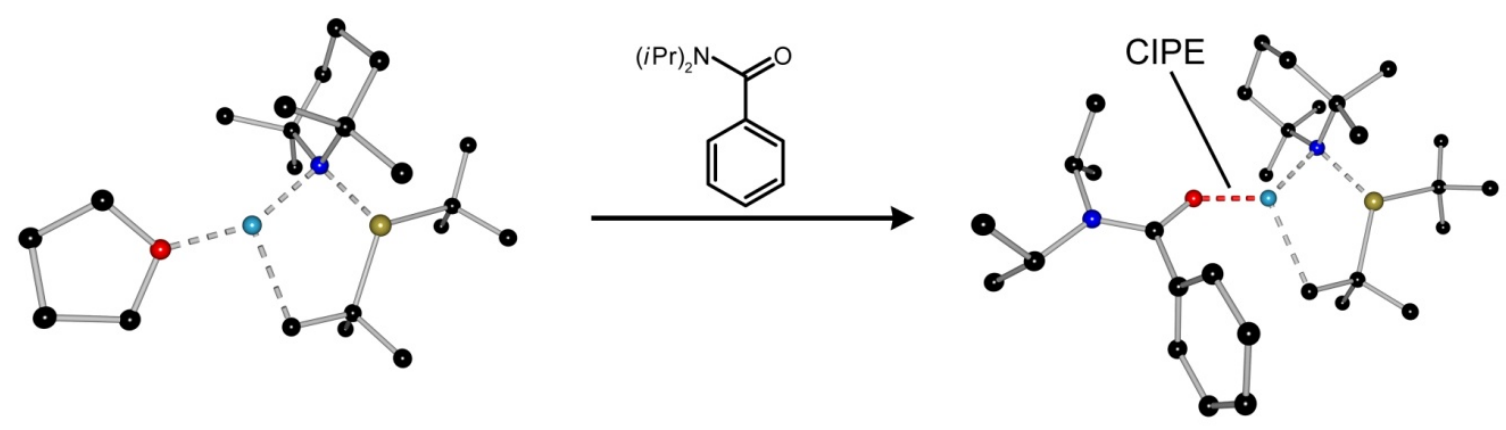

Scheme 4-5. CIPE complex for the ortho zincation of $N, N$-diisopropylbenzamide. ${ }^{[158]}$

Even if there is a lack of complementing kinetic data for the AMMZ reaction, this precomplex seems to be at least a key intermediate on the reaction path. The final orthozincated structures were also obtained for both the reactions with the lithium and the sodium zincate base. ${ }^{[160]}$ Interestingly, two completely different molecular structures were obtained. In the events of the sodium zincate the course of reaction proceeds as anticipated. The ortho-zincated product features the already known backbone with one tBu group replaced by the metalated phenyl ring (figure 4-3, right). 

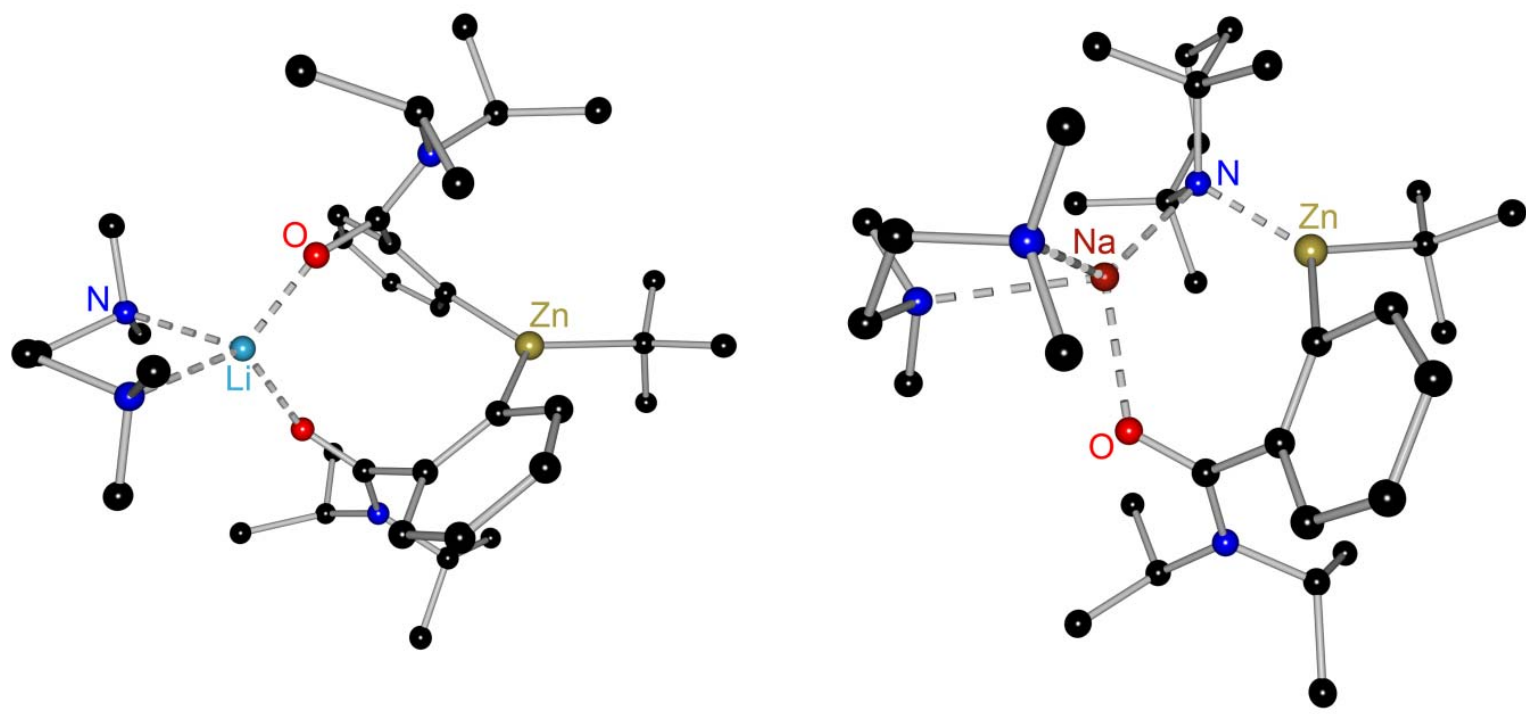

Figure 4-3. Molecular structures of twofold (left) and onefold (right) ortho-zincated $N, N$ diisopropylbenzamide. ${ }^{[160]}$

The contact to the sodium atom is achieved via the oxygen donor atom of the functional group closing a seven-membered (NaNZnCCCO) ring. In contrast, the lithium zincate accomplishes deprotonation of two equivalents of benzamide resulting in a totally diverse structure, which core is composed of a ten-membered [ $\mathrm{Li}(\mathrm{OCCC})_{2} \mathrm{Zn}$ ] ring (figure 4-3, left). Hereby two ortho zincated benzamides occupy the bridging positions between zinc and lithium.

Yet mechanisticially even more relevant is the observation that in the sodium structure $\mathrm{tmp}$ is preserved while in the lithium structure it is not. In general, mixed amido zincates exhibit both alkyl basicity and amido basicity. In view of the elucidated crystal structures so far where the alkyl ligand has been repelled in favor of the amide base it might errenous be concluded that these reagents function as an alkyl base. However, these findings were already contradicted by Kondo's original work, which suggests the metalated benzamides to be $R_{\text {aryl }}(t B u)_{2} Z n L i$ on the basis of NMR data. Not until comprehensive computational studies have been performed clarification on this discrepancy has been reached. ${ }^{[161]}$ By comparing the relative energies of products and educts DFT calculations confirm the general thermodynamic preference for an overall alkyl transfer. However, an examination of the whole reaction sequence reveals unexpected results that draw a mechanistically more precise picture (scheme 4-6). Initial formation of a pre-metalation complex should provide a proximity between the deprotonation agent and the substrate hydrogen to be abstracted. In this adduct two orientations PC1 and PC2, the first facilitating deprotonation by the alkyl base and the second deprotonation by the amido base, are possible. While the energy differences between PC1 and PC2 are still negligible, on approaching the transition states TS1 and TS2, 
a remarkably higher activation energy for the deprotonation with the alkyl base is encountered. On the contrary, the final product formation step is more exothermic for the alkyl than for the amido base.

\section{alkyl base}

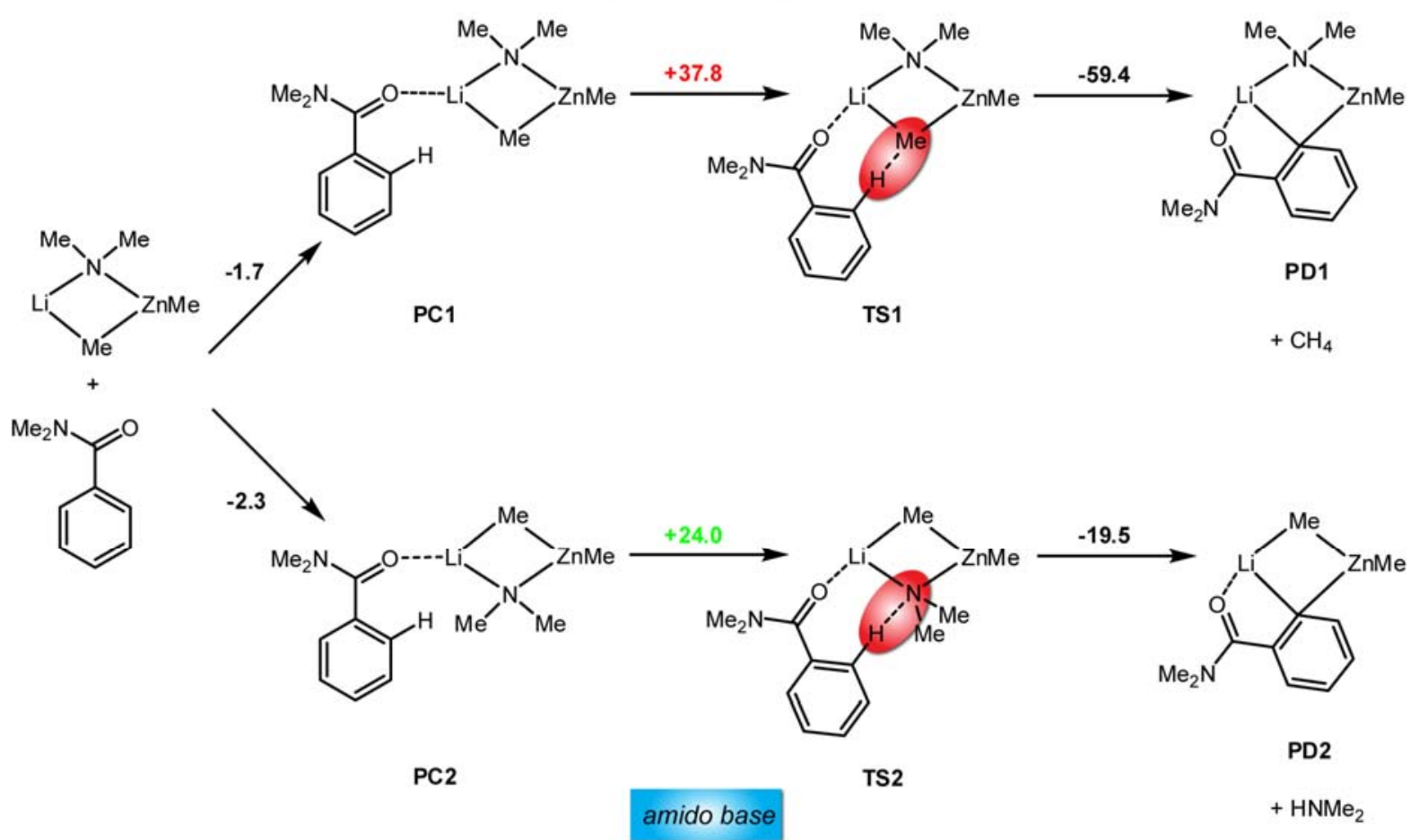

Scheme 4-6. Kinetic and thermodynamic reactivity of amidozincates. ${ }^{[161]}$

These data clearly show that the overall deprotonation with the alky base is the thermodynamically winning way. However, the activation energies suggest a strong kinetic preference for the amido base. In fact further calculations manifest the impression of a stepwise ortho deprotonation where tmp-Li acts as the kinetic base. In a subsequent step PD2 reacts with $\mathrm{HNMe}_{2}$ to give the final thermodynamic favored product PD1 and methane gas is produced.

Finally one of the most impressive examples of mixed metal synergy is the 'meta deprotonation' of functionalized aromatic compounds, complementing the established directed ortho metalation (DoM) by Snieckus et al.. ${ }^{[22,23,91]}$ Mulvey first accomplished to metalate $\mathrm{N}, \mathrm{N}$ dimethylaniline employing the amido zincate $\left[(\text { tmeda}) \mathrm{Na}\left(\mu-{ }^{t} \mathrm{Bu}\right)(\mu-\mathrm{tmp}) \mathrm{Zn}^{\mathrm{t}} \mathrm{Bu}\right)^{[162]}$ as well as toluene using $[(\operatorname{tmeda}) \mathrm{Na}(\mu-\mathrm{Bu})(\mu-\mathrm{tmp}) \mathrm{Mg}(\mathrm{tmp})]^{[163]}$ in the previously inaccessible metaposition. It is especially worth mentioning that during the deprotonation of toluene the thermodynamically more acidic methyl group hydrogens are not abstracted and the formation of a resonance stabilized carbanion is not observed. The reaction with $N, N$-dimethylaniline proceeds also contrary to initial predictions. The dimethylamino group is known to exhibit an ortho-directing effect in metalation reactions with monometallic phenyl- and butyllithium 
reagents. ${ }^{[164,165]}$ On the contrary, regioselective meta deprotonation is observed when the heterobimetallic tmp zincate base is employed (figure 4-4). ${ }^{[162]}$ The fail for the directing effect to appear is probably due to the fact that the nitrogen lone pair of the functional group occupies a p-orbital which is engaged in conjugation with the $\pi$-system of the phenyl ring.

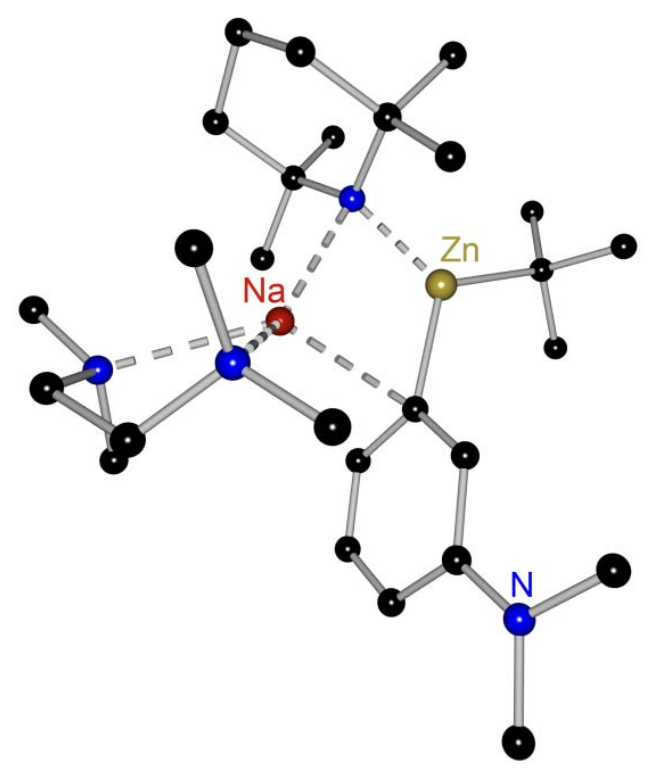

Figure 4-4. N,N-dimethylaniline zincated in meta position. ${ }^{[162]}$

All AMMZ reactions have in common that both metals have to be in close proximity. This is essential for synergistic effects to become feasible. Thus a contact ion pair between the negatively charged organozinc fragment and the positive alkali metal counterion can be anticipated in solution which is inert towards dissociation. Obviously the situation is completely different for the classical triorganozincates. As mentioned, one way for them to be formed consists in the disproportionation of diorganozincates as it occurs in the presence of polycyclic macrocycles. ${ }^{[151]}$ The resulting active species responsible for the increase in reactivity is a homometallic solvent separated $R_{3} Z^{-}$species. Similarly the reaction of diorganozinc reagents with organolithium nucleophiles in the presence of tmeda yields solvent separated ion pairs (figure 4-5). ${ }^{[166]}$ If the donor ligand is omitted, dissociation into the alkyllithium and the dialkylzinc species occurs. ${ }^{[167]}$ Thus not only the superiority of higher kinetic basicity of the tmp ligand over the retarded reactivity of the alkyl ligand are responsible for the enhanced possibilities of mixed amidozincates over classical trialkylzincates. Completely different molecular structures (CIP vs. SSIP) are possible, which should influence the reaction behavior to a much greater extent. 


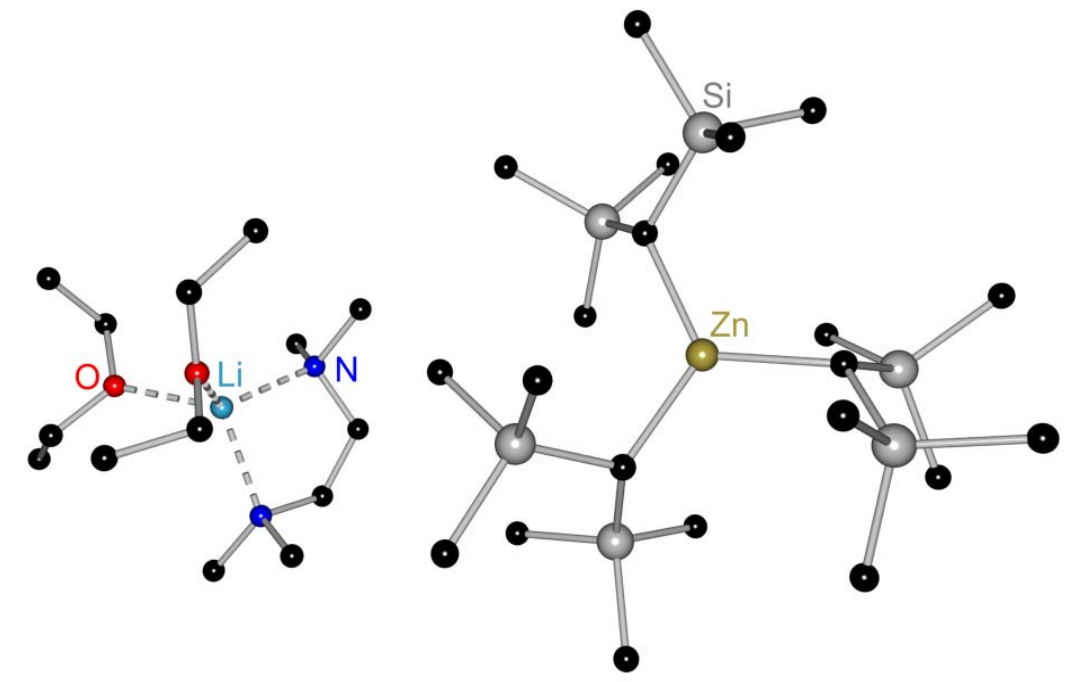

Figure 4-5. Molecular structure of the solvent seperated lithium-tris-[bis(trimethylsilyl)methyl]zincate $\cdot \mathrm{tmeda}^{2} 2 \mathrm{Et}_{2} \mathrm{O} .{ }^{[166]}$

Zincate agents play an important role in synthetic chemistry and the great diversity of reactions (by switching from alkylzincates to mixed amidozincates) makes them a valuable target for analytical chemists. Unfortunately, structural information on this generally highly reactive organometallic compounds is only available in form of systems which are stabilized by bulky substituents ( $t \mathrm{Bu}, \mathrm{SiMe}_{3}$, phenyl etc.), which exhibit a shielding effect on the reactive metal center. Admittedly, these compounds are less air and moisture sensitive and therefore easier to isolate, but the information on the molecular architecture gained cannot be transferred unequivocally to highly reactive ate complexes containing smaller alkyl ligands. This is due to the fact that the steric demand of stabilizing ligands can change the structure of the parent ate compound making it impossible to deduce reasonable reactivity patterns from these model compounds. Since our working group is specialized in the handling of highly sensitive compounds and their crystal application at ultra-low temperature, authentic structural information for the parent compound of triorganozincates, lithium trimethylzincate, could be elucidated and the influence of neutral donor ligands investigated.

\subsubsection{SSIPs and a CIP of parent Trimethylzincate}

Dimethylzinc in toluene was added to a solution of methyllithium in diethyl ether at $0{ }^{\circ} \mathrm{C}$. After one hour of stirring a white precipitate formed. 1.5 eqivalents of diglyme were added to the solution (scheme 4-7). After additional stirring for one hour the solution was filtrated under protective atmosphere and the resultant solution was stored at $-45^{\circ} \mathrm{C}$. Highly reactive crystals grew over a period of one week that were suitable for an X-ray analysis. 


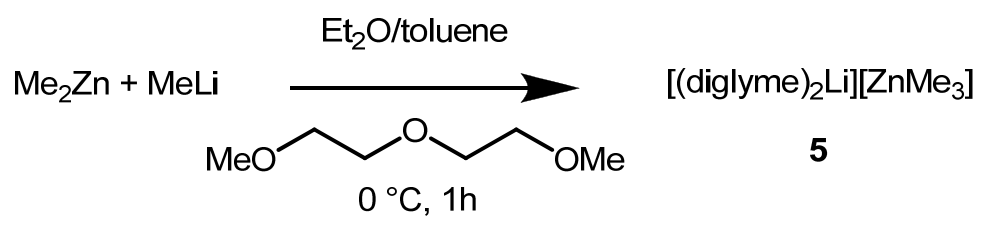

Scheme 4-7. Synthesis of 5.

The crystals were applied to the diffractometer at $-100^{\circ} \mathrm{C}$. At slightly higher temperatures they decompose rapidly. The crystal structure consisted of the solvent separated complex [(diglyme $\left.)_{2} \mathrm{Li}\right]\left[\mathrm{ZnMe}_{3}\right](5)$ that crystallizes in the monoclinic space group $P 2_{1} / c$. (Figure 4-6). The zinc atom is coordinated by three methyl groupsto give a trigonal planar environment. The lithium ion is coordinated by the six oxygen atoms of the two almost orthogonal diglyme molecules forming a slightly distorted octahedral coordination polyhedron. 5 represents the first SSIP structure revealing the undisturbed $\mathrm{Me}_{3} \mathrm{Zn}^{-}$anion. The $\mathrm{Zn}-\mathrm{C}$ bond lengths are identical within the estimated standard deviations (202.8(5) to 203.4(4) pm, table 4-2). They are somewhat shorter than zinc-carbon bonds in Weiss's $\mathrm{Me}_{4} \mathrm{ZnLi}_{2}(207.1 \mathrm{pm}){ }^{[156]}$ where tetra-coordination of zinc and the additional negative charge lead to stronger repulsion between the four methyl ligands. However, the 1968 determined bond lengths contain some variance because of high estimated standard deviations (esds) and an uncertainty in the assignment of the correct space group. In the triorganozincate $\left.\left[(\text { tmeda })_{2} \text { (thf }\right)_{2} \mathrm{Li}\right]\left[\mathrm{Zn}\left\{\left(\mathrm{CH}\left(\mathrm{SiMe}_{3}\right)_{2}\right\}_{3}\right]^{[166]}\right.$ the zinc-carbon contacts are even longer (av. $208.9 \mathrm{pm}$ ). Obviously the bulk of the trimethylsilylgroups is a strong factor for the ligand metal distance in zincates while the extension of the coordination sphere in the case of small methyl ligands has a slightly reduced impact. The sum of $\mathrm{C}-\mathrm{Zn}-\mathrm{C}$ angles $\left(359.99^{\circ}\right)$ witnesses that the $\mathrm{Me}_{3} \mathrm{Zn}^{-}$anion is essentially planar.

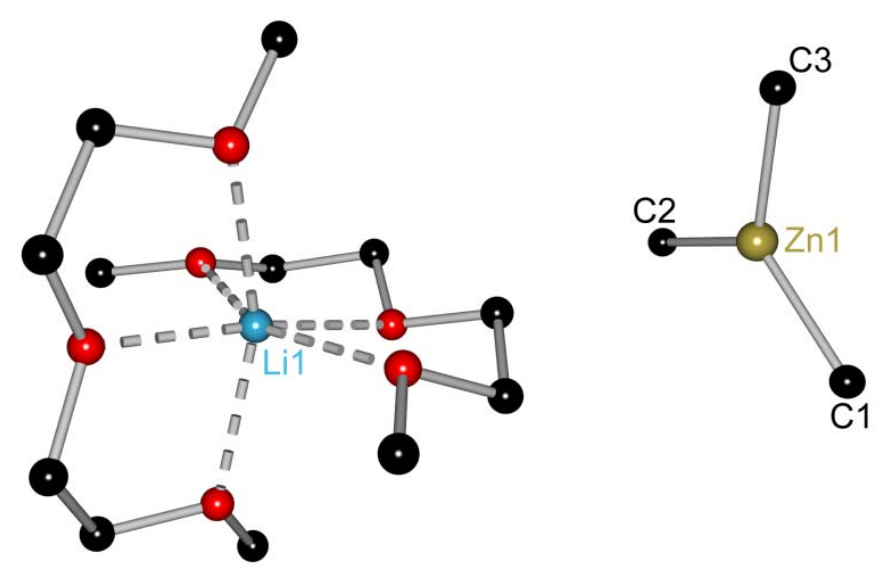

Figure 4-6. Molecular structure of [(diglyme $\left.)_{2} \mathrm{Li}\right]\left[\mathrm{ZnMe}_{3}\right](\mathbf{5})$. 
Table 4-2. Selected bond lengths $[\mathrm{pm}]$ and angles $\left[{ }^{\circ}\right]$ for $\left[(\text { diglyme })_{2} \mathrm{Li}\right]\left[\mathrm{ZnMe}_{3}\right](\mathbf{5})$ and related compounds.

\begin{tabular}{|c|c|c|c|}
\hline & $\begin{array}{c}{\left[(\text { diglyme })_{2} \mathrm{Li}\right]\left[\mathrm{ZnMe}_{3}\right]} \\
\mathbf{( 5 )}\end{array}$ & $\mathrm{Me}_{4} \mathrm{ZnLi}{ }_{2}^{[156]}$ & $\begin{array}{l}{\left[(\text { tmeda })_{2}(\text { thf })_{2} \mathrm{Li}\right]} \\
{\left[\mathrm{Zn}\left\{\left(\mathrm{CH}\left(\mathrm{SiMe}_{3}\right)_{2}\right\}_{3}\right]^{[166]}\right.}\end{array}$ \\
\hline $\mathrm{Zn} 1-\mathrm{C} 1$ & $202.8(5)$ & av. 207.1. & $208,5(9)$ \\
\hline Zn1-C2 & $203.4(4)$ & & $209,2(9)$ \\
\hline Zn1-C3 & $203.1(4)$ & & $209,1(9)$ \\
\hline $\mathrm{C} 1-\mathrm{Zn} 1-\mathrm{C} 2$ & $122.08(18)$ & av. $108.5^{\circ}$ & $120.4(4)$ \\
\hline $\mathrm{C} 2-\mathrm{Zn} 1-\mathrm{C} 3$ & $116.91(18)$ & & $119.6(4)$ \\
\hline C3-Zn1-C1 & $121.00(18)$ & & $118.7(4)$ \\
\hline
\end{tabular}

At otherwise the same conditions the reaction was carried out in the presence of the tridentate amine ligand pmdeta. While in the case of diglyme a SSIP was generated, this time a CIP was isolated (scheme 4-8).

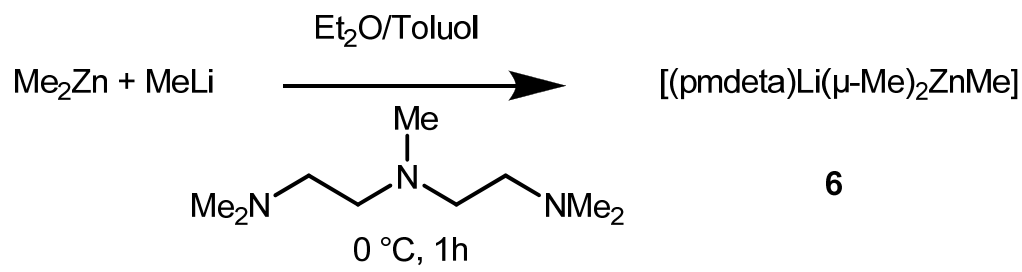

Scheme 4-8. Synthesis of 6.

[(pmdeta)Li( $\mu-\mathrm{Me})_{2} \mathrm{ZnMe}$ (6) crystallizes in the orthorhombic space group Pbca. (figure 47). A central four-membered ( $\left.\mathrm{LiC}_{2} \mathrm{Zn}\right)$-ring is the structural motif. Surprisingly, both $\mathrm{Li}-\mathrm{C}$ bond lengths vary considerably. While the $\mathrm{Li}-\mathrm{C} 1$ distance of $232.2(3) \mathrm{pm}$ is in the normal range considered in lithiumorganics, the $\mathrm{Li}-\mathrm{C} 2$ bond of $271.0(3) \mathrm{pm}$ is at the far end of that range (table 4-3). Taking these distances into account it might be anticipated that the closer bond to $\mathrm{C} 1$ has some impact on the $\mathrm{Zn}-\mathrm{C}$ bonds of the trigonal planar $\mathrm{ZnMe}_{3}$ moiety. In fact the closer $\mathrm{Li}-\mathrm{C} 1$ bond gives rise to the longest $\mathrm{Zn}-\mathrm{C} 1$ bond of 205.7(2) pm, compared to the pending Zn-C3 bond of 201.8(2) pm. Obviously, the competition of two electropositive metals for the electron density of the methanide elongates the zinc-carbon bond. The lithium coordination widens the $\mathrm{C} 1-\mathrm{Zn}-\mathrm{C} 2$ angle to $122.13(7)^{\circ}$. 


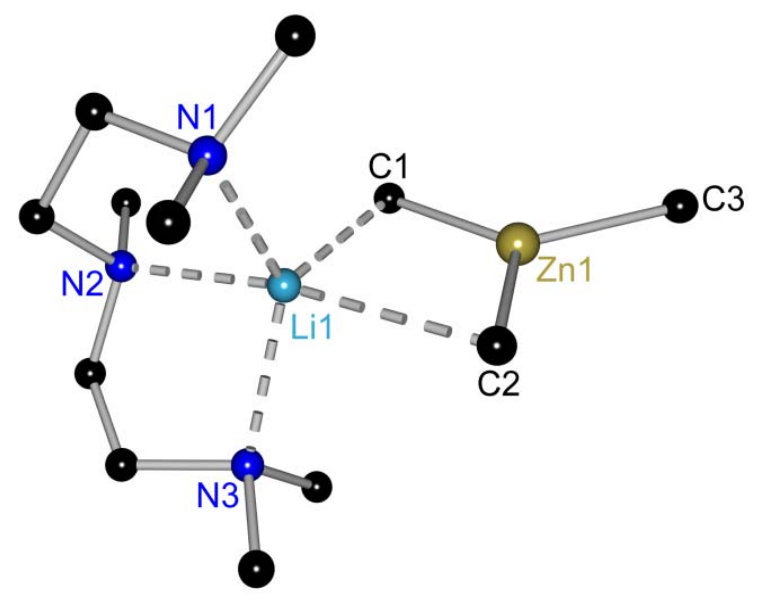

Figure 4-7. Molecular structure of 6.

A similar four-membered $\left(\mathrm{LiC}_{2} \mathrm{Zn}\right)$-ring is found in the structure of the related higher coordinated zincate [(tmeda) $\left.{ }_{2} \mathrm{Li}_{2} \mathrm{ZnMe}_{4}\right]$ which was published in 2008 by Hevia et al. (figure 4-8). ${ }^{[168]}$ The Li-C distances there (219.3(5)-225.1(5) pm) are more balanced and 7-12 pm shorter than the respective close contact in 6 . The smaller $\mathrm{C}-\mathrm{Zn}-\mathrm{C}$ angles of the tetraorganozincate $\left(\operatorname{av} 109.46^{\circ}\right)$ enable a tighter symmetric coordination of lithium. Concerning the carbon-zinc bonds a distinct elongation in Hevia's zincate can be seen (av. $212.9 \mathrm{pm}$ ). The difference of these distances between tri- and tetraorganozincates seem to be more pronounced for the CIP than for the SSIP structures.

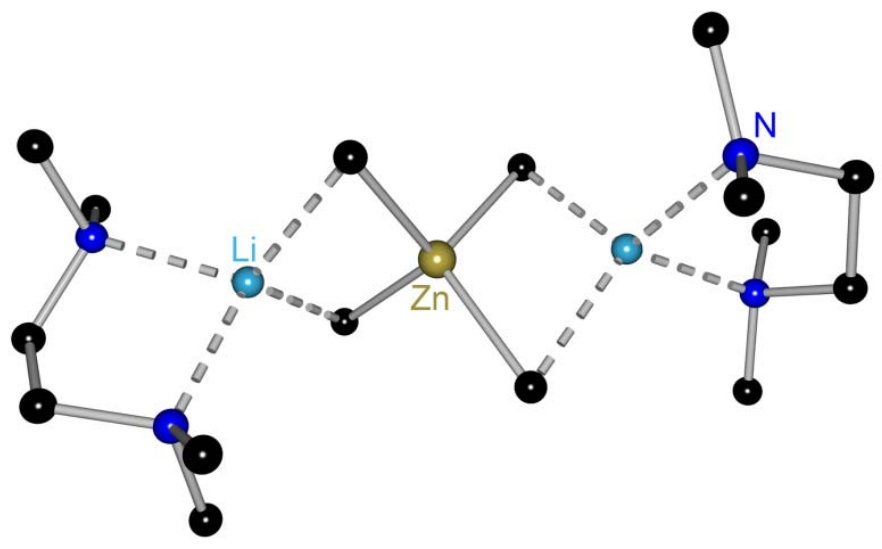

Figure 4-8. Molecular structure of [(tmeda $\left.)_{2} \mathrm{Li}_{2} \mathrm{ZnMe}_{4}\right] .{ }^{[168]}$

It is also interesting to make a comparison to the lithium-carbon distances of uncomplexed $\mathrm{Me}_{4} \mathrm{ZnLi}_{2}$. These are between 252 and $284 \mathrm{pm}$ and thus far longer than the corresponding short distances in $\left[(\text { tmeda })_{2} \mathrm{Li}_{2} \mathrm{ZnMe}_{4}\right]$ and 6. Obviously the lithium carbon distances in zincates exhibit a great variance while the changes in the zinc-carbon bonds 
are less pronounced. This supports the assumption that the interaction to the alkali metal is the key factor for the reactivity of these compounds.

Table 4-3. Selected bond lengths $[\mathrm{pm}]$ and angles $\left[{ }^{\circ}\right]$ for $\left[(\right.$ pmdeta $\left.) \mathrm{Li}(\mu-\mathrm{Me})_{2} \mathrm{ZnMe}\right](\mathbf{6})$ and a related compound.

\begin{tabular}{l|ll}
\hline & {$\left[(\right.$ pmdeta $\left.) \mathrm{Li}(\mu-\mathrm{Me})_{2} \mathrm{ZnMe}\right](6)$} & {$\left[(\text { tmeda })_{2} \mathrm{Li}_{2} \mathrm{ZnMe}_{4}\right]^{[168]}$} \\
\hline Zn1-C1 & $205.7(2)$ & $211.8(3)$ \\
Zn1-C2 & $203.9(2)$ & $212.0(3)$ \\
Zn1-C3 & $201.8(2)$ & $213.5(2)$ \\
Zn1-C4 & - & $214.3(3)$ \\
Li1-C1 & $232.2(3)$ & \\
Li2-C2 & $271.0(3)$ & \\
N1-Li1 & $215.1(3)$ & \\
N2-Li1 & $222.7(3)$ & \\
N3-Li1 & $220.8(11)$ & av.109.46 $210.05(5)$ \\
C1-Zn1-C2 & $122.13(7)$ & \\
C2-Zn1-C3 & $118.88(8)$ & \\
C3-Zn1-C1 & $118.99(7)$ & \\
\hline
\end{tabular}

At attempts to synthesize the tmeda analogon of 6 by reacting MeLi with $\mathrm{Me}_{2} \mathrm{Zn}$ in a $1: 1$ ratio in hexane Hevia encountered an interesting behavior. Although the previously mentioned NMR studies by Mobley and Berger revealed that in thf solution the trimethyzincate is more stable than the highly coordinated zincate, only crystals of the latter were obtained. Theoretical studies modeling the reaction of of MeLi with $\mathrm{Me}_{2} \mathrm{Zn}$ in the presence of tmeda yielded an energy gain of $-19.98 \mathrm{kcal} / \mathrm{mol}$. However, subsequent addition of a second equivalent of MeLi is also exothermic by $-14.88 \mathrm{kcal} / \mathrm{mol}$. Therefore the synthesis of the hypothetical triorganozincate $\left[(\right.$ tmeda $\left.) \mathrm{Li}(\mu-\mathrm{Me})_{2} \mathrm{ZnMe}\right]$ failed. The fact that the respective ate complexes $\mathbf{5}$ and $\mathbf{6}$ were successfully obtained now in the presence of the tridentate donors pmdeta and diglyme shows that is possible to control not only the formation of CIP vs. SSIP but also the equilibrium between $\mathrm{Me}_{3} \mathrm{ZnLi}$ and $\mathrm{Me}_{4} \mathrm{ZnLi}_{2}$. 
The solid state structures of organometallics obtained from X-ray data are not necessarily consistent with the molecular structures in solution. Sometimes just the least soluable species crystallizes and the solvent hull being replaced by the crystal environment can induce substantial changes in molecular geometry, too. Furthermore, in solution different aggregation states might appear in superimposed equilibria, ${ }^{[150]}$ where the most reactive species is just the minor component. To compare the solid state and solution structures of $\mathbf{5}$ and 6 we performed ${ }^{1} \mathrm{H}$ Diffusion ordered NMR experiments (DOSY). ${ }^{[169,170]}$

It was found that the diffusion constants of the [(Diglyme $\left.)_{2} \mathrm{Li}\right]^{+}$cation $\left(D=-8.73 \log \left(\mathrm{m}^{2} / \mathrm{s}\right)\right)$ and the $\left[\mathrm{ZnMe}_{3}\right]^{-}$anion $\left(D=-8.59 \log \left(\mathrm{m}^{2} / \mathrm{s}\right)\right)$ in 6 are different, indicating no interaction between the anion and the cation in the SSIP in solution (figure 4-9).

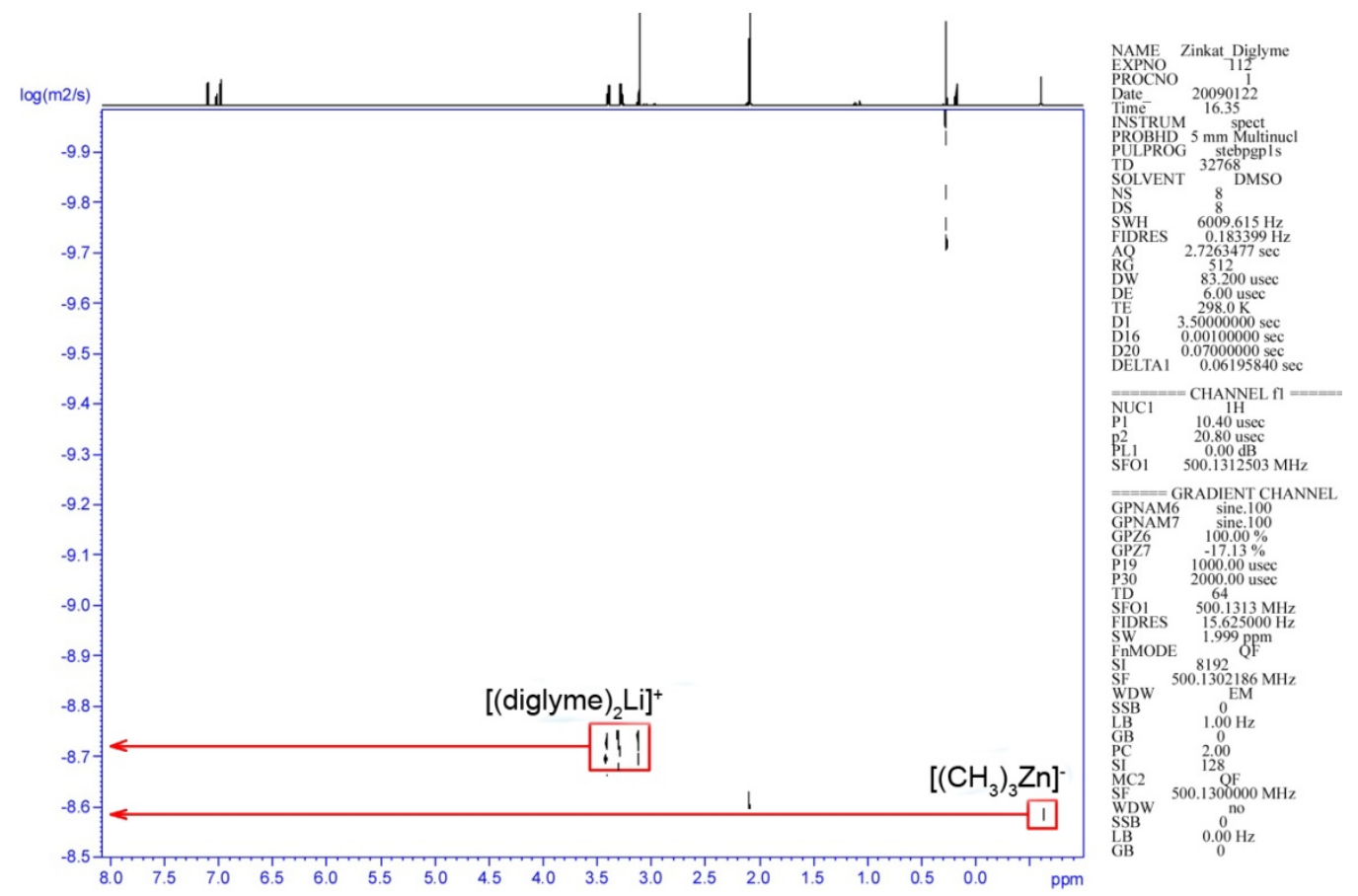

Figure 4-9. DOSY of 5.

Of greater importance though is the question whether the CIP of $\mathbf{5}$ is preserved in solution or whether solvent molecules can break the cluster apart. The ${ }^{1} \mathrm{H}-\mathrm{NMR}$ sprectra show only one singlett for the protons of the $\left[(\mathrm{Me})_{3} \mathrm{Zn}\right]$-group indicating the rapid exchange of the Mepositions or even an SSIP in solution. However, the diffusion constants of pmdeta and $\mathrm{ZnMe}_{3}$ were determined to be identical $\left(D=-8.92 \log \left(\mathrm{m}^{2} / \mathrm{s}\right)\right.$, proving that the CIP is retained in solution (figure 4-10). 


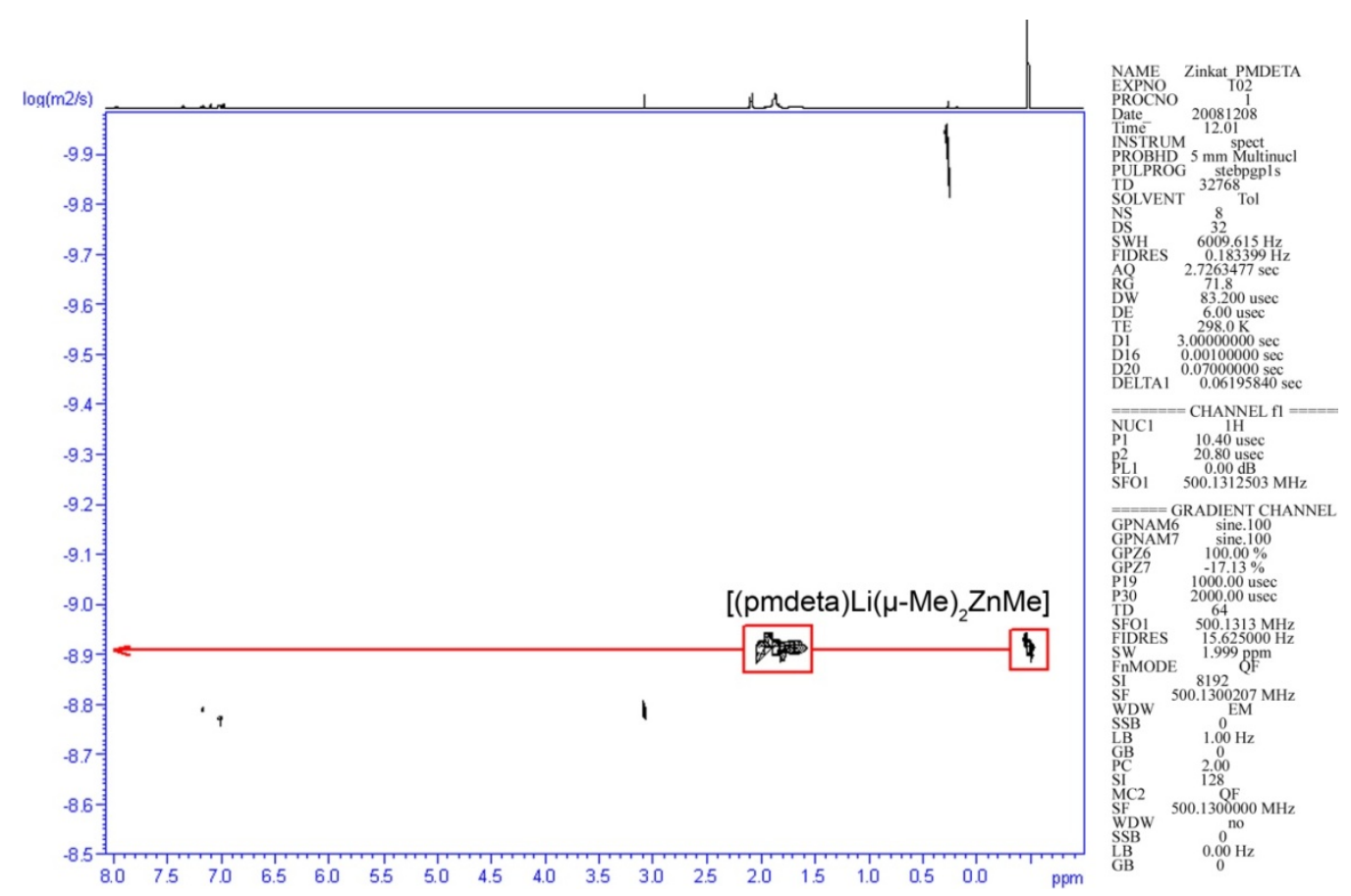

Figure 4-10. DOSY of 6.

As expected, the trend of the diffusion constants, correlated to the size of the particles, is in tune with the aggregates along $\left[(\right.$ pmdeta $\left.) \mathrm{Li}(\mu-\mathrm{Me})_{2} \mathrm{ZnMe}\right]>\left[(\text { diglyme })_{2} \mathrm{Li}\right]^{+}>\left[\mathrm{ZnMe}_{3}\right]^{-}$. Hence, both DOSY experiments prove that the structural information from the single crystal diffraction experiments mirror the aggregation state of zincates $\mathbf{1}$ and $\mathbf{2}$ even in solution, which is essential for the prediction of reasonable reactivity patterns.

An interesting salt effect on the formation of the CIP $\left[(\right.$ pmdeta $\left.) \mathrm{Li}(\mu-\mathrm{Me})_{2} \mathrm{ZnMe}\right](6)$ could be obtained accidently. Surprisingly, one reaction following the procedure to synthesize 6 yielded crystals of a different composition. It turned out that traces of lithium chloride impurities must have been present which have been incorporated in the molecular structure (scheme 4-9).

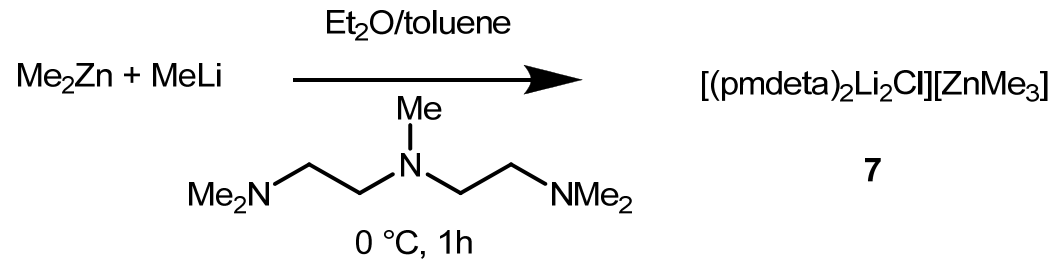

Scheme 4-9. Unexpected synthesis of 7.

[(pmdeta $\left.)_{2}\left(\mathrm{Li}_{2} \mathrm{Cl}\right)\right]\left[\mathrm{ZnMe}_{3}\right](7)$ crystallizes in the space group $P 2_{1} / n$. Fascinatingly LiCl cocomlexation is able to destroy the CIP. Usually pmdeta is unable to coordinate lithium with two equivalents, but the presence of $\mathrm{LiCl}$ leads to the formation of a $\mathrm{Li}-\mathrm{Cl}-\mathrm{Li}$ fragment which both ends are terminated by a pmdeta ligand. The chlorine in the middle keeps both pmdeta molecules apart so no steric interference can occur (figure 4-11). 

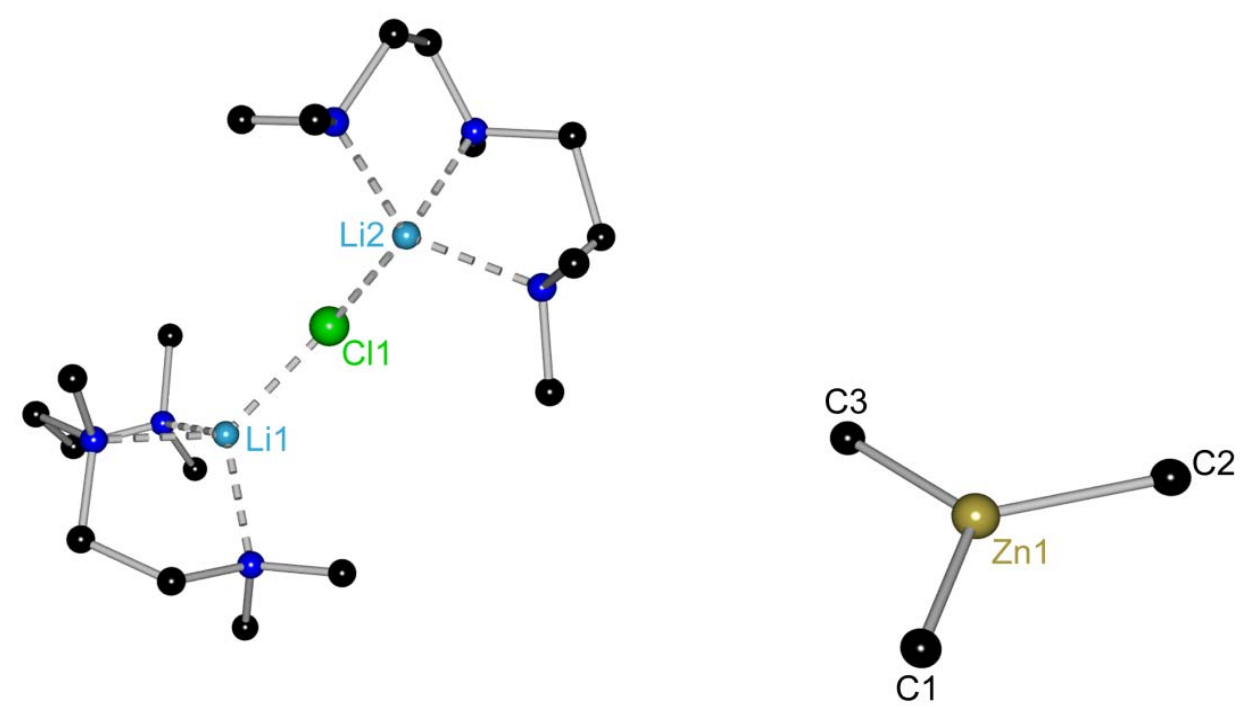

Figure 4-11. Molecular structure of $\left[(\text { pmdeta })_{2}\left(\mathrm{Li}_{2} \mathrm{Cl}\right)\right][\mathrm{ZnMe} 3](7)$

The zinc-carbon distances are in the range of 203.6(3) and 204.1(3) pm (table 4-4) and thus nearly identical to SSIP 5.

Unfortunately selective formation of 7 could not be reproduced by the addition of either one equivalent or excess solid lithium chloride.

Table 4-4. Selected bond lengths $[\mathrm{pm}]$ and angles $\left[^{\circ}\right]$ for $\left[(\text { pmdeta })_{2}\left(\mathrm{Li}_{2} \mathrm{Cl}\right)\right]\left[\mathrm{ZnMe}_{3}\right](7)$.

\begin{tabular}{|c|c|c|c|}
\hline \multicolumn{3}{|c|}{$\left[(\text { pmdeta })_{2}\left(\mathrm{Li}_{2} \mathrm{Cl}\right)\right]\left[\mathrm{ZnMe}_{3}\right](7)$} & \multirow{2}{*}{$\frac{\left[(\text { pmdeta })_{2}\left(\mathrm{Li}_{2} \mathrm{Cl}\right)\right]\left[\mathrm{ZnMe}_{3}\right](7)}{119.59(11)}$} \\
\hline $\mathrm{Zn} 1-\mathrm{C} 1$ & 203.6(3) & C2-Zn1-C3 & \\
\hline Zn1-C2 & 203.9(3) & C3-Zn1-C1 & $120.49(11)$ \\
\hline Zn1-C3 & $204.1(3)$ & Li1-Cl1 & $225.1(4)$ \\
\hline $\mathrm{C} 1-\mathrm{Zn} 1-\mathrm{C} 2$ & $119.92(11)$ & Li2-Cl1 & $228.0(4)$ \\
\hline
\end{tabular}

\subsubsection{Conclusion}

The molecular precomplex formed between a Lewis acidic and a Lewis basic organometall compound is of fundamental importance for the reactivity of ate reagents. This precomplex can exist either as a SSIP or a CIP. Since especially the latter has drawn much attention because of the exertion of cooperative bimetallic effect, the control of selective formation is irreplaceable. It could be demonstrated that this is achievable exclusively by choice of the appropriate donor base. Mixing dimethylzinc and methyllithium in the presence of pmdeta or diglyme yields selectively the triorganozincate. While diglyme induces the formation of a SSIP, pmdeta is able stabilize the CIP (scheme 4-10). The sensitivity of these 
building preferences could be shown on the crystal structure of $\left[(\text { pmdeta })_{2}\left(\mathrm{Li}_{2} \mathrm{Cl}\right)\right]\left[\mathrm{ZnMe}{ }_{3}\right](7)$, where traces of lithium chloride were able to destroy the CIP in favor of a SSIP.

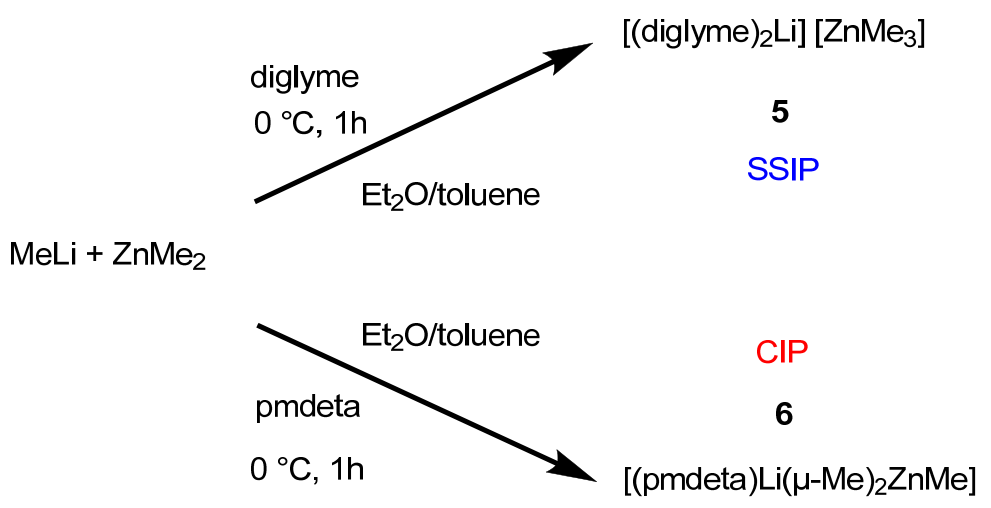

Scheme 4-10. Control of selective formation of a CIP vs. SSIP of trimethyzincate.

\subsection{Computational Studies on Zincates}

\subsubsection{Formation preferences of CIP vs. SSIP}

Heterobimetallic ate bases open routes to new unexpected products through sophisticated reaction mechanism. Their complex composition and the manifold possibilities to aggregate make it indispensable to establish knowledge on the exact formation principles. The experimental results represented in the previous chapter displayed that it can be a difficult task to predict the actual structure resulting on mixing the different precursor reagents although profound experience on their monometallic congeners is available. Therefore theoretical DFT calculation about the thermodynamic stability of possible precomplexes can be helpful in the development of new reagents. To see whether the outcome of the experimental results could have been forecast this way the relative energies of modeled CIPs and SSIPs of trimethylzincate were envisaged. First the hypothetical CIP of $\mathrm{Me}_{3} \mathrm{ZnLi}$ with diglyme was compared to the SSIP. Both CIP and SSIP were modeled and optimized with the B3LYP/6-31g basis set. By subtracting the energy of educts calculated separately on the same level of theory, a relative stabilization of $-171.98 \mathrm{~kJ} / \mathrm{mol}$ was obtained for the CIP. For the SSIP, which consists of one equivalent $\mathrm{Me}_{3} \mathrm{ZnLi}$ and two equivalents of diglyme, the same procedure gave a relative stability of $-193.64 \mathrm{~kJ} / \mathrm{mol}$ (scheme $4-11$ ). Of course the stability refers to gas phase structures, but in apolar solvent like hexane, where no competeting solvation effects against the strong donor bases employed should occur, the SSIP is clearly favourable. 


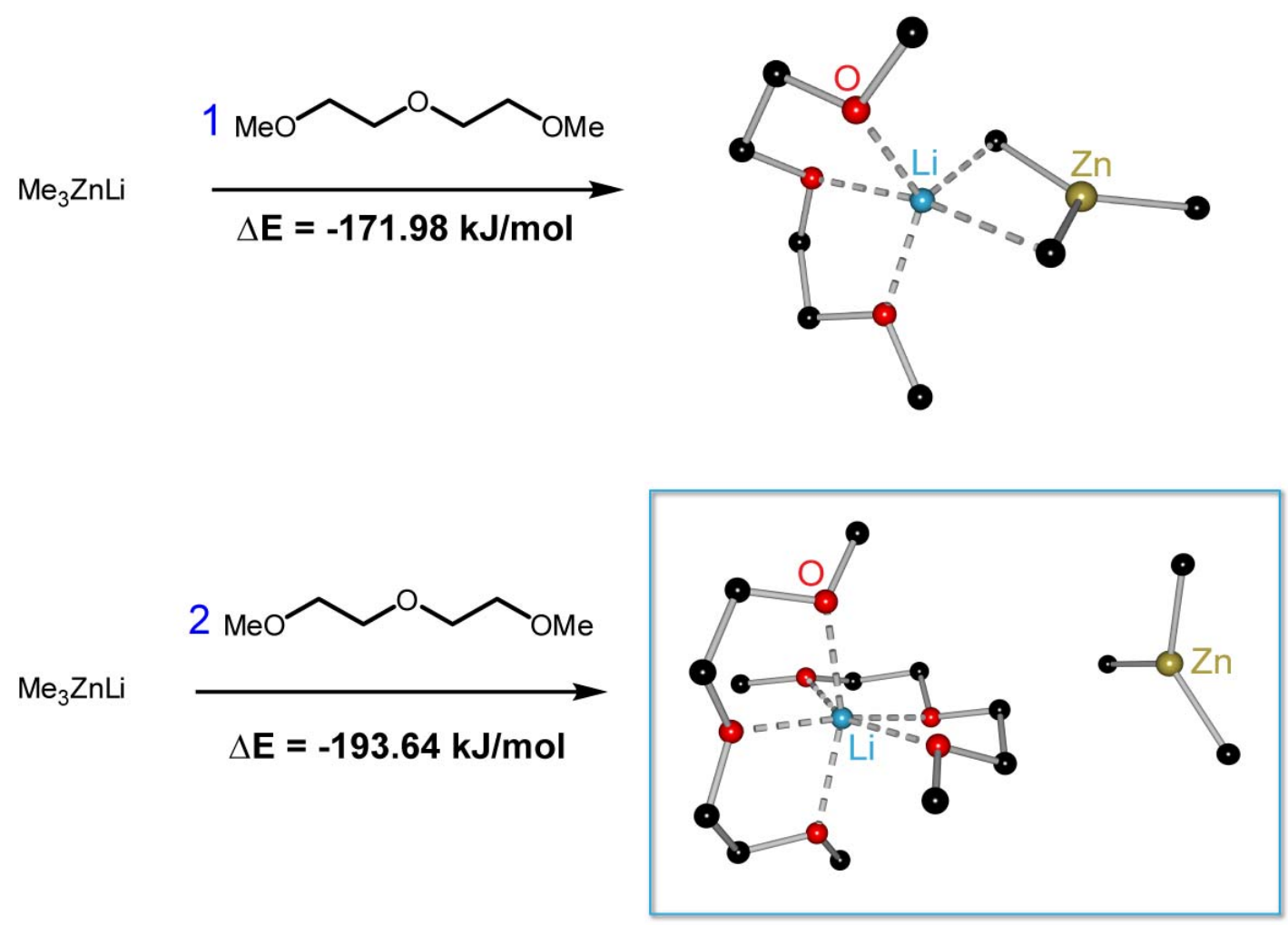

Scheme 4-11. Comparison of the relative stabilization energies of [(diglyme $\left.) \mathrm{Li}(\mu-\mathrm{Me})_{2} \mathrm{ZnMe}\right]$ and [(diglyme $\left.)_{2} \mathrm{Li}\right]\left[\mathrm{ZnMe}_{3}\right]$.

When comparing the CIP and the hypothetical SSIP of $\mathrm{Me}_{3} \mathrm{ZnLi}$ and tmeda, the situation is even clearer. The contact ion pair yielded a relative stability of $-166.86 \mathrm{~kJ} / \mathrm{mol}$, which is comparable to that of the calculated CIP with diglyme. But when trying to optimize the hypothetical SSIP, no stable minimum on the potential energy surface (PES) could be detected. During the course of optimization, one of the two pmdeta ligands coordinating to lithium is pushed away leaving lithium complexed by one pmdeta ligand only (scheme 4-12). Presumably the energy of three additional lithium-nitrogen contacts does not compensate for the huge amount of steric repulsion arising between the bulky $\mathrm{NMe}_{2}$ groups. This repulsive effect seems to be distinctly smaller for the sterically less demanding methoxy groups of diglyme. Hence the observed experimental results could be completely confirmed by computational studies. 

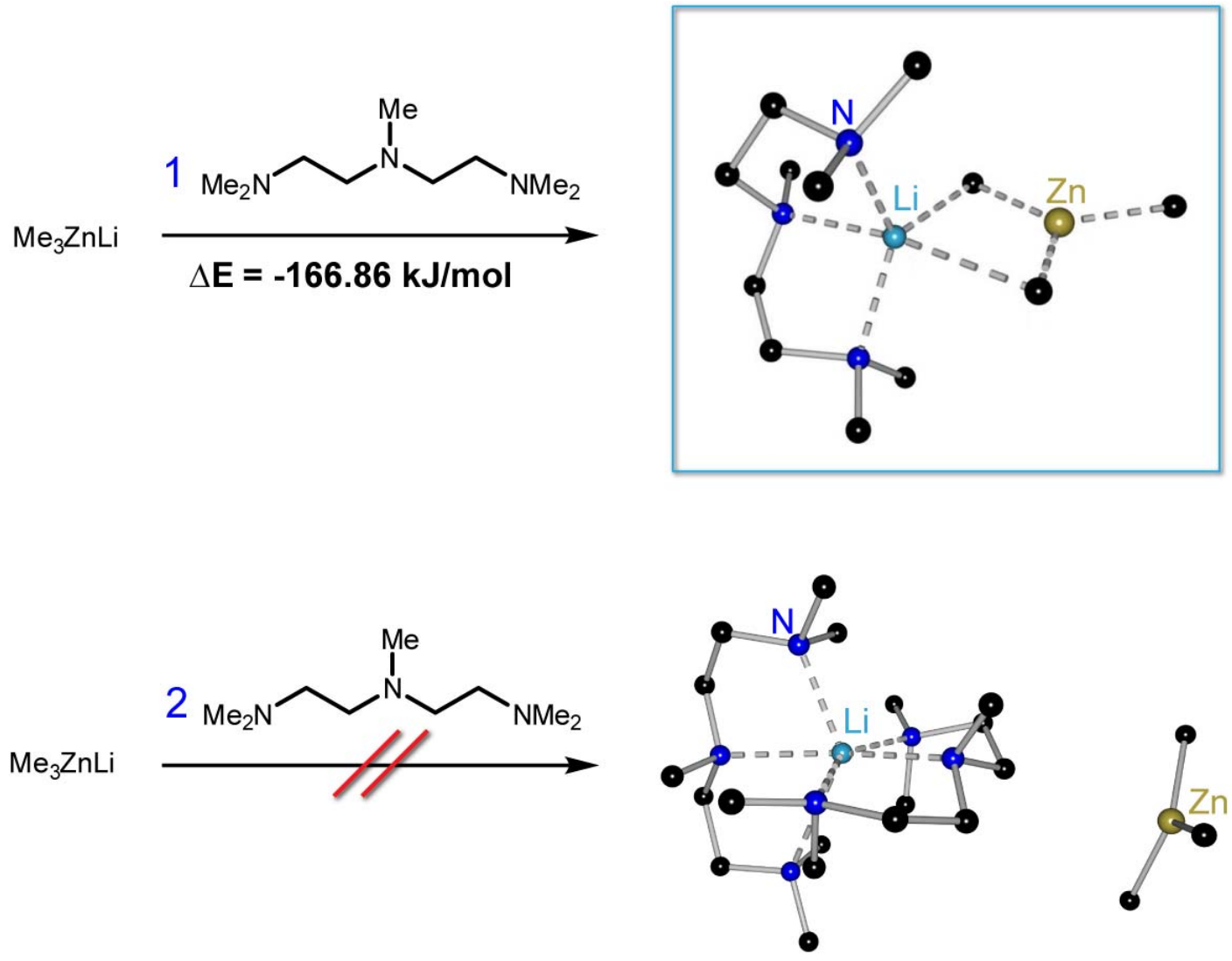

no stable minimum on PSE

Scheme 4-12. Comparison of the relative stabilization energies of $\left[(\right.$ pmdeta $\left.) L i(\mu-M e)_{2} \mathrm{ZnMe}\right]$ and $\left[(\text { pmdeta })_{2} \mathrm{Lij}_{[}\left[\mathrm{ZnMe}_{3}\right]\right.$.

\subsubsection{Evaluation of bonding properties}

After performing calculations on the building preferences of CIPs and SSIP, is seemed worth to analyze to bonding situation in the relevant CIP in more detail. Since these bimetallic reagents often outperform there monometallic congeners, there must be some cooperative effect between both metal atom centers, which should be detectable by analyzing the ED distribution with Bader's QTAIM. ${ }^{[73]}$

A series of calculations with the intension to monitor the change in bonding properties started from the neutral dimethyl zinc. Afterwards the diorganozinc compound was compared to the corresponding free $\mathrm{Me}_{3} \mathrm{Zn}^{-}$anion, which can serve as a model for the SSIP anion. In the last step the bonding properties in $\left[(\right.$ pmdeta $\left.) \mathrm{Li}(\mu-\mathrm{Me})_{2} \mathrm{ZnMe}\right](6)$ were analyzed in order to find a reason for the extraordinary reactivity of CIPs.

The first question to be tackled concerned the polarity of the metal-carbon bond. Can it be described better in terms of covalency or in terms or is the interaction predominantly electrostatic? The low reactivity of organozinc reagents compared to organolithium or magnesium bases was ascribed to the substantial covalent character of the zinc-carbon bond. ${ }^{149]}$ Even though today the higher reactivity of $\mathrm{C}-\mathrm{Li} / \mathrm{Mg}$ bonds is explained by the 
greater ionicity, it has to be noted that there was also a long dispute on the nature of the bonding in organolithium compounds. ${ }^{[5,6]}$ Since these dissolve well in apolar media like hexane it was argued that they should exhibit some covalent character. However, this phenomenon could be explained by the formation of oligomers with a polar lithium core surrounded by lipophilic alkyl groups which interact with the solvent. Today the carbon lithium bond is considered to be mainly ionic. ${ }^{[5]}$ But since the electronegativity of zinc (1.65) is much higher than that of lithium (0.98) and even magnesium (1.37), in fact a distinct level of covalency in the zinc-carbon bond can be anticipated.

Frenking et al. analyzed the bonding in organozinc compounds with the NBO (Natural Bond Orbitals ${ }^{[70]}$ method and QTAIM. ${ }^{[171]}$ Interestingly, the NBO analysis of dimethylzinc yielded only one metal-carbon orbital with $96 \%$ s-character. Obviously the zinc atom is not sp hybridized as expected from the linear geometry of the molecule. Translated into the Lewis picture this means that $\mathrm{Me}_{2} \mathrm{Zn}$ can be best described as an overlay of two resonance formulas with only one zinc-carbon bond (scheme 4-13). The positive calculated atomic charge of zinc $(1.33 \mathrm{e})$ suggests a strong polarity of the bond.

\section{$\mathrm{MeZn}^{+} \mathrm{Me}^{-} \rightleftharpoons \mathrm{Me}^{-} \mathrm{ZnMe}^{+}$}

Scheme 4-13. Mesomeric forms of dimethylzinc.

To see how the situation changes when adding a methanide anion, the ED of dimethylzinc (8) and trimethylzincate (9) was computed with Turbomole. When choosing an appropriate basis set some considerations have to be undertaken. First in anionic compounds often the negative charge is not well localized near one nucleus but delocalized over a huge area. Second when performing a topological analysis according to QTAIM, we are not interested in a good description of the core electrons, but the outer electrons since they are responsible for the bonding. Since atomic basis sets are optimized in minimizing the energy, it is always more effective to add additional gaussian functions for the description of core electrons than for the description of valence electrons, since electrons near the nucleus contribute stronger to the atomic energy and this is what is minimized during a structure calculation. Because of these reasons normally diffuse functions are necessary for this kind of purposes. Unfortunately diffuse functions are computationally very expensive. Therefore instead of using a split valence set plus diffuse functions, the bigger Ahlrich's triple zeta basis set TZVP ${ }^{[97]}$ was used. The TZVP basis set is slightly better than the $6-311 \mathrm{G}^{* *}$ basis set and still faster to compute than a smaller basis set with diffuse functions. To exclude functional dependencies the BP86 ${ }^{[172,64]}$ as well as the hybrid functional B3LYP ${ }^{[64,66]}$ were used. For the converged structures the electron density was calculated on a grid with the mesh size 0.1 Bohr with DGRID-4.4. 
A subsequent topological search for bond critical points yielded all anticipated BCPs for the zinc-carbon bonds. The density at the $\mathrm{BCP}$ in $\mathrm{Me}_{2} \mathrm{Zn}\left(0.75 \mathrm{e} \AA^{-3}\right)$ is $27 \%$ higher than in the $\mathrm{Me}_{3} \mathrm{Zn}$ anion $\left(0.59 \mathrm{e}^{-3}\right)$. Higher values generally correspond to a stronger sharing of electrons between nuclei, indicating that the metal-carbon bonds in the neutral dimethylzinc are indeed slightly more covalent than in the triorganozincate. Thereby the absolute values are located between $\rho\left(\mathbf{r}_{\mathrm{BCP}}\right)$ of typical carbon-carbon single bonds $\left(\sim 1.7 \mathrm{e} \AA^{-3}\right)^{[73]}$ and carbon-lithium bonds $\left(\sim 0.15 \mathrm{e} \AA^{-3}\right) \cdot{ }^{[98]}$ Unfortunately transferring the properties at the BCP from one molecule to another is only exactly valid when the atoms connected by a bond path are the same, so here these values give just a rough impression.

Further information of the polarity of the bond can be gathered by evaluating the values for the Laplacian at the BCP. These are $5.09 \mathrm{e}^{-5}$ in dimethylzinc (8) and $5.18 \mathrm{e} \AA^{-5}$ in trimethylzincate. In both cases the curvature is positive which is typical for a polar bond, in which electron density is not concentrated in the bonding region but shifted towards the more electronegative atom. This is also reflected by the calculated charges. The zinc atom in $\mathrm{Me}_{2} \mathrm{Zn}(8)$ has an AIM charge of +0.77 e while the carbon atoms bear a negative charge of 0.46 e each. In $\mathrm{Me}_{3} \mathrm{Zn}^{-}(9)$ the differences are nearly the same, zinc possessing a charge of +0.80 e and carbon -0.43 e (table 4-4).

Besides the discussion of values for the BCP and atomic charges, the situation can be visualized by contour plots of the Laplacian distribution. Herby a distinct region of charge depletion around the zinc nucleus and an accumulation of charge on the carbon atoms can be observed for 8 as with 9 (figure 4-12). The BCP is shifted towards central metal atom and thus settled in a region of charge depletion. On an isosurface value of -0.35 VSCCs on carbon directed towards the zinc cation can be detected, which can be interpreted as a $\mathrm{sp}^{3}$ hybridized lone pair. The above results imply that zinc-carbon bonds in the ate complex do not differ from that in the diorganozinc species in general, but the values for $\rho\left(r_{B C P}\right)$ suggest a weakening of bond strengths for the zincate. This is accompanied by an increase of bond lengths (196.4 pm to $209.0 \mathrm{pm}$ ) in the gas phase also. 

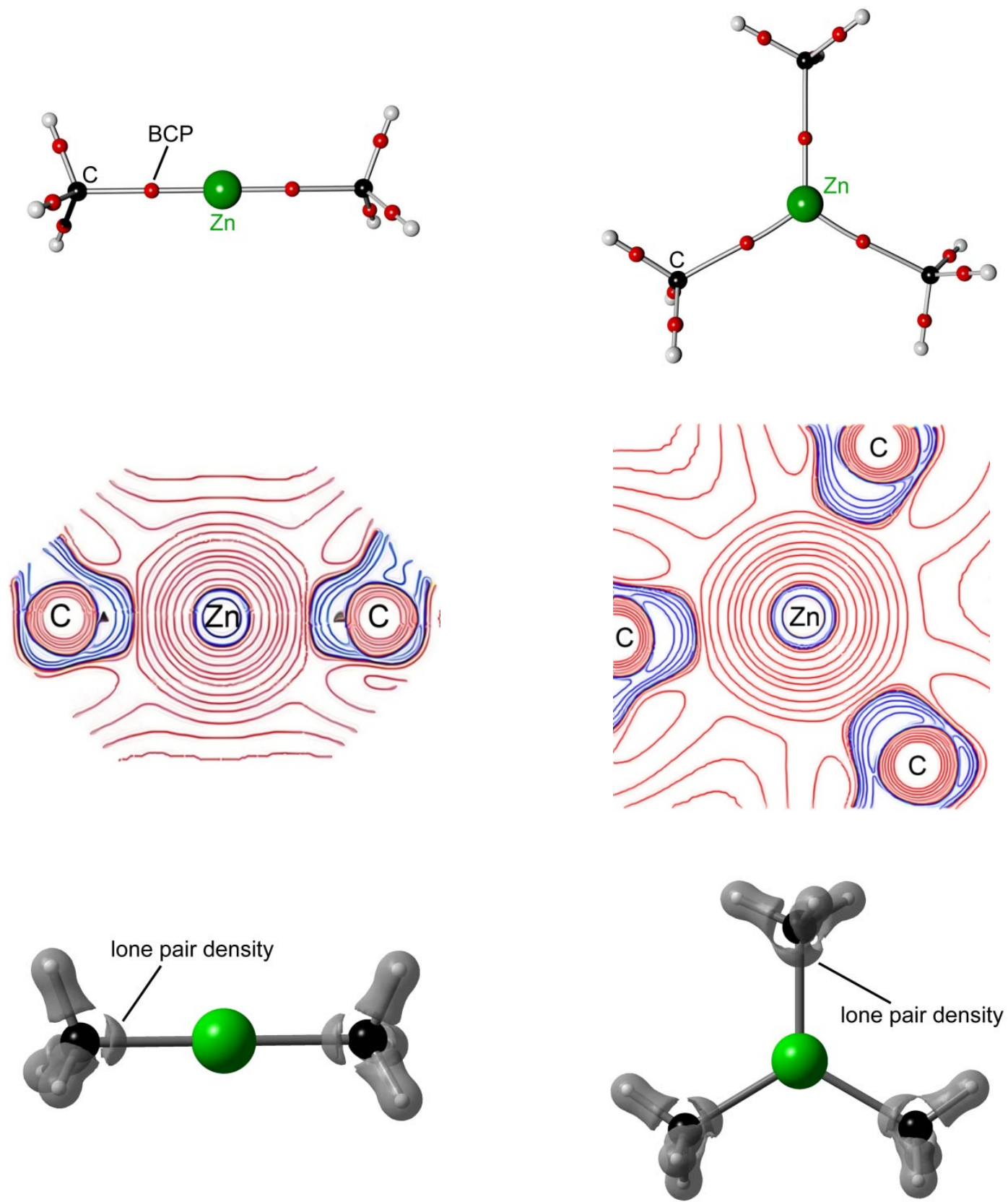

Figure 4-12. Molecular graphs, Laplacian contour maps and Laplace isosurfaces at $-0.35 \mathrm{e} / \AA^{5}$ for dimethylzinc (8, left) and trimethylzincate (9, right).

Summarizing it can be concluded that firstly the additional charge in the ate complex definitely plays a role for the higher reactivity. Although it is delocalized over three methyl groups so each one bears only a slightly increased charge compared to the divalent $\mathbf{8}$, the overall negative charge should play an important electrostatic attraction to electrophiles in total. Secondly the weakened bond strength in $\mathbf{9}$ should ease the transfer of one methyl group to the acceptor substrate.

Independent NBO analysis with gaussian03 on the b3pw91/b3pw91/6-311+ level of theory are in accordance to the QTAIM results. The NBO charges are even higher, being +1.26 for 
zinc and -1.29 for carbon in dimethylzinc and $+1.41 /-1.34$ e in trimethylzincate respectively, pronouncing the strong polarity of the bond. While absolute charge values, which depend not only on the employed method but also on the level of theory, should always be treated with care, the observed trend - the charge separation being higher in $\mathrm{Me}_{3} \mathrm{Zn}^{-}$than in $\mathrm{Me}_{2} \mathrm{Zn}-$ remains intact (table 4-5).

Table 4-5. Selected QTAIM and NBO properties for dimethylzinc (8) and trimethylzincate (9).

\begin{tabular}{l|ll}
\hline & dimethylzinc (8) & trimethylzincate (9) \\
\hline$\rho\left(\mathbf{r}_{\mathrm{BCP}}\right)\left[\mathrm{e} \AA^{-3}\right]$ & 0.75 & 0.57 \\
$\nabla^{2} \rho\left(\mathrm{r}_{\mathrm{BCP}}\right)\left[\mathrm{e}^{-5}\right]$ & 5.09 & 5.18 \\
Q AIM Zn & +0.77 & +0.80 \\
Q AIM C & -0.46 & -0.43 \\
Q NBO Zn & +1.26 & +1.41 \\
Q NBO C & -1.29 & -1.34 \\
NBO Zn-C & Occ. 2.00 e, & Occ 1.99 e, \\
& Zn 95.4 \% s-character & Zn 96.5\% s-character \\
\hline method/ & Optimization and topology: Turbomole bp/TZVP \\
basis set & Optimitation and NBO analysis: Gaussian03 b3pw91/6-311+ \\
\hline
\end{tabular}

To come back to Frenking, who proposed a bond order of 0.5 for the zinc-carbon bond, the situation was also analyzed for $\mathrm{Me}_{3} \mathrm{Zn}^{-}$for comparison. Surprisingly in both dimethylzinc and the zincate anion exists only one bonding metal-carbon orbital with an occupation of two electrons. The s-character is $96.5 \%$ for 9 and $95.4 \%$ for $\mathbf{8}$. Obviously, the bond order in the trimethylzincate anion is less than one and consequently the best Lewis representation of this molecule is given by three mesomeric forms (scheme 4-14).

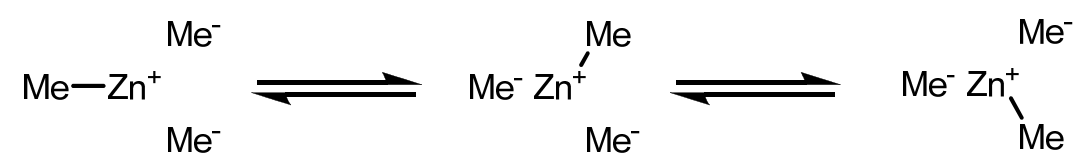

Scheme 4-14. Mesomeric forms of trimethylzincate.

To characterize the bonding in $\left[(\right.$ pmdeta $\left.) \mathrm{Li}(\mu-\mathrm{Me})_{2} \mathrm{ZnMe}\right](4)$ and to substantiate the Li-C agnostic contact in the CIP a gas phase optimization at the B3LYP/TZVP level of theory was performed. The results exclude that the contact originates from fuzzy crystal packing effects. A topological analysis of the electron density employing Bader's quantum theory of atoms in molecules (QTAIM) approach ${ }^{[73]}$ was carried out. In an energy minimized gas phase structure optimization the overall molecular graph was reconstructed starting from the crystal geometry apart from a marginal twist of the $\mathrm{ZnMe}_{3}$ unit (figure 4-13). The theoretical $\mathrm{Li}-\mathrm{C} 1$ bond length 
(233.3 pm) is close to the experimental distance of $231.9(3) \mathrm{pm}$, while the Li-C2 distance of $262.7 \mathrm{pm}$ even is shorter than the experimental (270.6(4) pm).

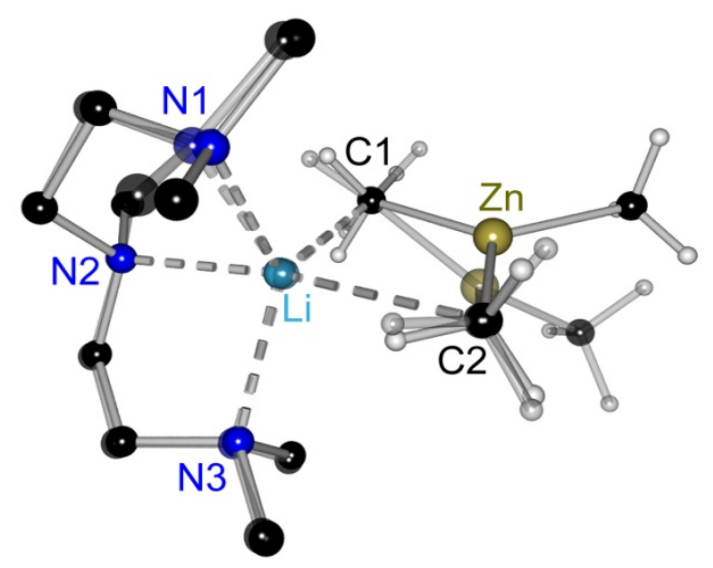

Figure 4-13. Superposition of the molecular structures of $[($ pmdeta $\left.)] \mathrm{Li}(\mu-\mathrm{Me})_{2} \mathrm{ZnMe}\right](\mathbf{6})$ derived from Xray experiment (solid) and geometry optimisation (transparent).

All anticipated BCPs, including the one at the $\mathrm{Li}-\mathrm{C} 1$ bond, could be found. However, there is no bond bath established between Li and C2 (figure 4-14). In the computed CIP the Zn-C distances are even more affected by the proximity to the lithium metal. While the methyl group at $\mathrm{C} 1$ has to share the density with both metals and the $\mathrm{Zn}-\mathrm{C} 1$ bond is elongated to $212.2 \mathrm{pm}$ the pending $\mathrm{Zn}-\mathrm{C} 3$ bond is about $10 \mathrm{pm}$ shorter.

The topological features of the metal-carbon bonds are in the range anticipated for organometallics: the density at the BCP is relatively low and the Laplacian is positive, indicating a polar bond. ${ }^{[173-178]}$ The electron density at the $\mathrm{Li}-\mathrm{C} 1$ bond of $0.11 \mathrm{e} \AA^{-3}$ is approximately as high as at the shorter $\mathrm{Li}-\mathrm{N}$ donor bonds $\left(0.12 \mathrm{e}^{-3}\right)$ and the density at the $\mathrm{Zn}-\mathrm{C}$ bond correlates with the distance: the shorter the bond the higher the density. The BCPs of the $\mathrm{Li}-\mathrm{N}$ and the $\mathrm{Li}-\mathrm{C}$ bonds are located closer to the lithium than to the nitrogen atoms and the carbon atom reflecting the differences in electronegativity.

Remarkably, in the CIP of parent lithium trimethyl zincate no additional amide ligand is required to bridge the two metal atoms and to activate the $\mathrm{Zn}-\mathrm{C}$ bond. Just the suitable donor base pmdeta leaves a coordination site open and maintains the electronic depletion at the lithium atom. 

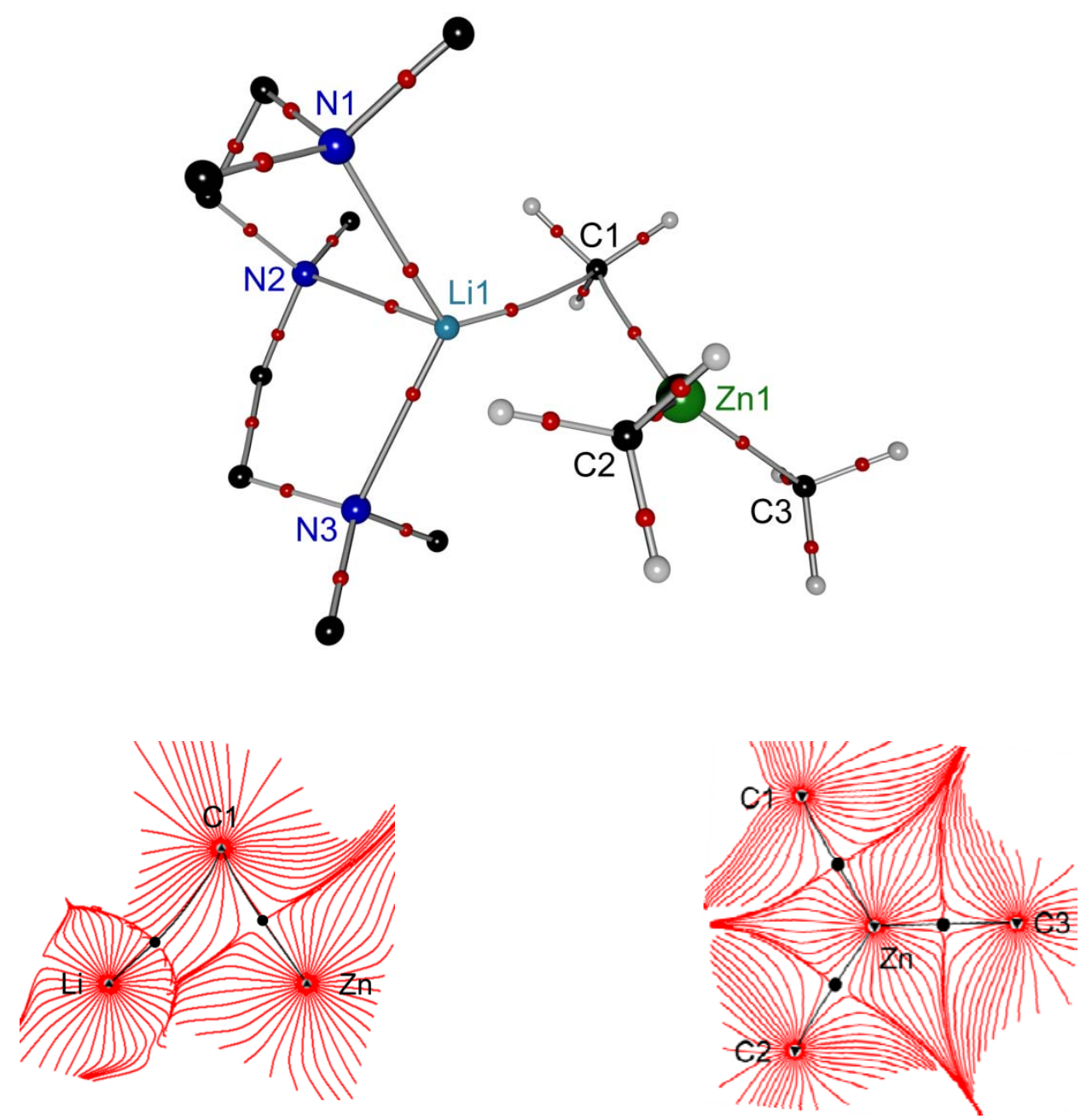

Figure 4-14. Molecular graph of 6 obtained from a gas phase optimization (B3LYP/TZVP). The small red spheres indicate BCPs. Trajectory plot (red lines) of the $\mathrm{Li}-\mathrm{C} 1-\mathrm{Zn}$ (left) and the $\mathrm{ZnMe}_{3}$ plane (right). The black lines indicate the bond paths. The black dots assign the BCPs.

Table 4-6. Bond distances $[p m]$, electron densities $\left[\mathrm{e} \AA^{-3}\right]$ and Laplacians $\left[\mathrm{e} \AA^{-5}\right]$ at the BCPs of the $\mathrm{Zn}-\mathrm{C}, \mathrm{C}-\mathrm{Li}$ and Li-N bonds.

\begin{tabular}{|c|c|c|c|}
\hline & $d$ & $\rho\left(\mathbf{r}_{\mathrm{BCP}}\right)$ & $\nabla^{2} \rho\left(\mathbf{r}_{\mathrm{BCP}}\right)$ \\
\hline C1-Li1 & $231.1(231.9)$ & 0.11 & 1.90 \\
\hline C1-Zn1 & $212.2(205.9)$ & 0.51 & 4.90 \\
\hline C2-Zn1 & $209.0(204.2)$ & 0.55 & 5.19 \\
\hline C3-Zn1 & $203.0(202.0)$ & 0.63 & 4.89 \\
\hline N1-Li1 & $224.1(215.1)$ & 0.12 & 2.49 \\
\hline N2-Li1 & $240.3(222.7)$ & 0.08 & 1.66 \\
\hline N3-Li1 & $226.2(220.8)$ & 0.12 & 2.37 \\
\hline
\end{tabular}




\subsubsection{Conclusion}

Computational studies have been performed to establish differences in the bonding of organozinc reagents and the SSIP and CIP of trimethyzincate. Combined QTAIM and NBO analyses revealed that the zinc-carbon bond actually exhibits a substantial covalent character, although NBO yielded only one bonding orbital for both dimethylzinc and trimethylzincate. Changes in the bond strength could be made visible by evaluating properties at the BCP. Thereby it was found that $\rho\left(r_{B C P}\right)$ decreases from $\mathrm{Me}_{2} Z n$ to $\mathrm{Me}_{3} Z^{-}$. A further weakening is observed for the zinc-carbon bond in the CIP which is involved in agostic bonding to the lithium. This is accompanied by an increase in bond length as well. This could activate the methyl group and improve its kinetic basicity in deprotonation reactions. 


\section{HYPERVALENCY}

\subsection{Evaluation of the bonding situation in formally hypervalent molecules}

The fifth chapter of this thesis goes historically one step back. So far, the electronic properties of modern organometallic reagents have been examined both by QTAIM and ELI to deduce reasonable reactivity patterns. Yet these relatively new tools for bonding analysis are perfectly suited to tackle a fundamental problem of bonding theories as well - the existence of hypervalent molecules, which do not obey the Lewis octet rule. In view of the tremendous popularity of the Lewis concept, these compounds have ever since regarded as exceptional and their development was partly hampered

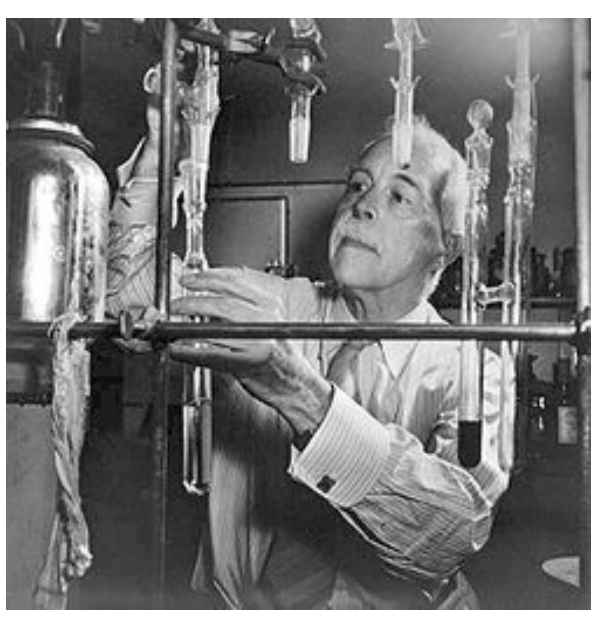

Figure 5-1. G. N. Lewis. since their synthesis was sometimes considered to be impossible. For this reason the chemistry of noble gas compounds has been slowed down, the first representative of it having been published not until $1962 .{ }^{[179]}$ Even though the violation of the octet rule could not be ignored at some stage, various bonding schemes (which are partly inconsistent with another) have been developed which convey the misleading idea that the bonding in these compounds is somehow different from that in well-behaving octet rule molecules. To understand the chronological development it is necessary to give a short survey of the Lewis concept in its original sense. ${ }^{[68]}$

At the beginning of the 20th century Lewis recognized that most of the known stable molecules posses an electronic structure with eight electrons in their valence shell. This feature is reflected by the "Aufbau" of the PSE, which tries to group elements with the same electronic valence structure and more or less similar properties together. When Lewis started to develop his bonding theory, quantum mechanics was still in its infancy and he proceeded on the assumption of the cubical atom model (figure 5-2).

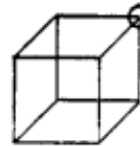

Li

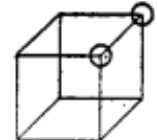

$\mathrm{Be}$

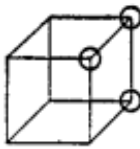

B

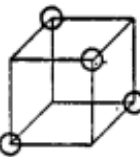

C

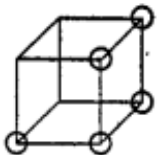

$\mathbf{N}$

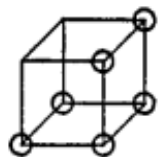

0

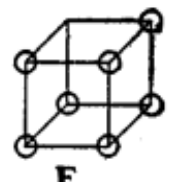

F

Figure 5-2. Representation of the atomic structure according to the cubical atom model. Picture taken from Lewis original publication. ${ }^{[68]}$ 
Hereby the atoms are separated into a kernel, which is composed of the nucleus and the inner shells, and the valence shell. It was recognized at that time that the kernel remains unaltered during chemical reaction whereas the outer shell configuration is responsible for an elements's reactivity. In the cubical atom model the valence electrons, which use to be mainly even numbers and in most cases eight, are believed to occupy the corners of a cube around the kernel. Fundamental quantum chemical statements like the Heisenberg uncertainty principle, leading to an indistinguishability of electrons, were still unknown, thus although the electrons were allowed to change place, it was stated that they are "held in position by more or less rigid constraints". ${ }^{[68]}$ In a molecule an atom consists of a positive charged kernel and a valence shell, which always possesses the charge -8 . Molecules were considered to be stable if the charge difference between kernel and valence shell is small. For example fluorine has a kernel charge of +7 . In a molecule fluorine acquires an electron octet, so the charge difference is +1 . If this difference exceeds four (e.g for alkali metals), the atom was considered to be unable to form covalent bonds. While Lewis believed unpolar molecules to obtain the electron octet by an equally sharing of electrons between them, these ionic compounds reach the closed valence shell by the transfer of electrons from one atom to the other. His mental image of the bond formation in the iodine molecule $I_{2}$ is depicted in figure 5-3.
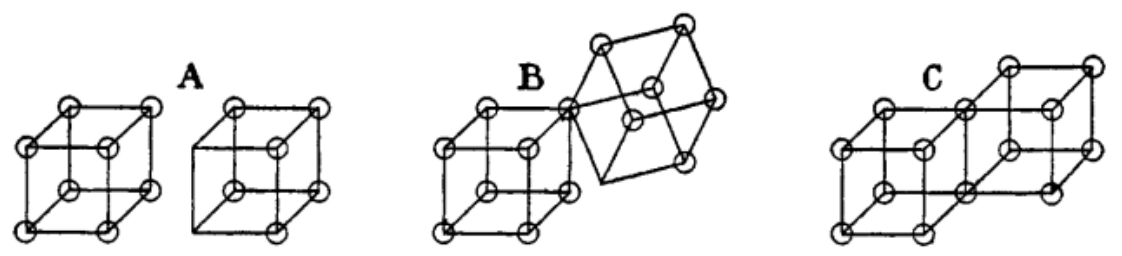

Figure 5-3. Three representations of the chemical bond in $\mathrm{I}_{2}$ Completely ionized (A), partly bound (B) and bound by a pair of electrons $(\mathbf{C})$. Picture taken from Lewis original publication. ${ }^{[68]}$

A represents the ionized form $\mathrm{I}^{-} \mathrm{I}^{+}$. On contrary establishment of a covalent bond can emerge when one atom contributes an electron sitting at a corner to the valence shell of the second atom (B). Vice versa the second atom can allow one electron to fill up the valence shell of the first atom (C), resulting in an electron octet for both iodine kernels. While a single bond is represented by an edge to edge link of two cubes, a double bond would be represented by a face to face connection.

This short introduction into Lewis original was intended to emphasize the state of chemical knowledge and the imagination of the chemical bond at that time. With this in mind the complications arising from hypervalent molecules become much clearer and less surprising. The octet rule was postulated before orbitals, hybridization schemes and many-center bonding theories have been developed. All the more it is surprising that especially the latter 
has been utilized to circumvent the concept of hypervalency and to proof the validity of a rule that is not based on quantum mechanics.

However, before discussing the limits of this empirical model, its tremendous usefulness in chemistry should be outlined. The description of chemical bonds in terms of electron pairs is still in our days a toolbox for chemists if they want to explain reactivities and properties of molecules. From the fundamental octet rule more sophisticated theories like the Lewis acidbase ${ }^{[180]}$ concept, the Klemm-Zintl concept ${ }^{[180]}$ and the VSEPR theory ${ }^{[181]}$ emerged. Especially the VSEPR theory is indispensable for the prediction of the 3-dimensional structure of many molecules by simply postulating, that electron pairs in a molecule should due to Pauli repulsion and electrostatics adopt a position of maximal distance to each other.

While most of the known molecules, especially in the first long row of the PSE, obey the octet rule, for second row elements bonding situations become more diverse. These higher homologous sometimes exhibit coordination numbers greater than four. On the assumption that each ligand atom is bonded to the central atom by a pair of electrons, which are equally shared between both nuclei, the valence shell electron population of the central atom exceeds eight. This phenomenon was termed hypervalency. First rationalizations for the bonding situation in these hypervalent molecules relied on the participation of empty $d$ orbitals, which were thought to be accessible for third-row elements. Hence, a valence expansion by the formation of $\mathrm{sp}^{3} \mathrm{~d}^{\mathrm{n}}$ ( $\mathrm{n}=1$ or 2 ) hybrid orbitals was postulated, so these elements can acquire up to 12 electrons in their outer shell. Prominent candidates are $\mathrm{PF}_{5}$ or $\mathrm{SF}_{6}$, but also sulfuric and phosphoric acid. In contrast, second row elements are limited to the formation of four $\mathrm{sp}^{3}$ hybrid orbitals, which can host a maximum of eight electrons..

However, the assumption, that the availability of energetically low lying d-orbitals yields an explanation for hypervalent molecules, had to be corrected in the 1980'. Theoretical results by Kutzelnigg et al. showed, that the energy gap between $p$ - and d-orbitals for third row elements becomes too big, making the involvement of d-orbitals in the hybridization highly unfavorable. ${ }^{[182]}$ The same is true for the energy required to promote an electron from a s- or p-orbital into an empty d-orbital. Therefore d-orbitals stay unoccupied in these classes of compounds. The only role, where d-orbitals are needed, is the use as polarization functions for $p$-orbitals in the quantum chemical calculations. These d-functions must not be confused with occupied orbitals, since their only purpose is to model the deformation of electron density because of chemical bonding. After d-orbitals were set ad acta, efforts have been made to show that hypervalent molecules in fact do not violate the octet rule. As the majority of the so-called hypervalent molecules possess ligands with a strong electonegativity, they were formulated by different resonance structures with one or more ionic bonds (figure 5-4, a and $\mathbf{b})$. 


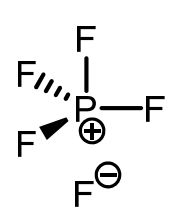

a

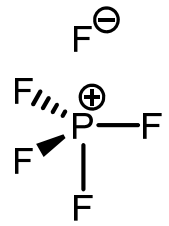

b

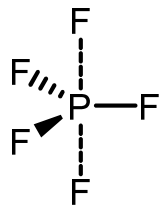

C

Figure 5-4. Different ionic resonance structures for $\mathrm{PF}_{5}(\mathbf{a}$ and $\mathbf{b})$ and description of the axial phosphor-fluorine bonds by 3-center-4-electron bonding (c).

Of course these resonance structures are now in accordance with the octet rule. However, as outlined above the Lewis rules are purely empirical, so there is no need to force hypervalent molecules in structure formulas that preserve the electron octet. A weakened form of this avoids the formulation of ionic bonds but incorporates the polarity of the bond, resulting in a to eight electrons reduced valence shell population of the central atom. Though, this moves away from the original octet rule, because now its fulfillment depends on the electronegativity of the ligands. On examples like $\mathrm{PMe}_{5}$, where the phosphorus valence shell population is 9.42 e as determined by the ELF, ${ }^{[183]}$ it can be seen, that the modified octet rule does not abolish the concept of hypervalency.

An alternative way to account for the bonding in hypervalent molecules without d-orbital participation are 3-center-4-electron bonds (figure 5-4, c). In this way the axial phosphorfluorine bonds in $\mathrm{PF}_{5}$ can be described as being formed between an unhybridized $\mathrm{p}$-orbital at phosphor which overlaps with the ligand orbitals of the fluorine atoms. In addition this bonding scheme features an explanation for the axial bond lengths increase in comparison with the equatorial P-F bonds. On the other hand it suggests that the bonding in the axial bonds is different in nature than in the equatorial bonds. Nevertheless, ELF calculations, which can make the connection of atoms in molecules visible by polysynaptic basins between those atoms, show no sign for a three center bonding in form of a trisynaptic basin. Instead, disynaptic basins are found between all phosphor and fluorine atoms. ${ }^{[184]}$ From this point of view it seems that the $\mathrm{P}-\mathrm{F}$ bonds in $\mathrm{PF}_{5}$ are not different in general and the elongation of axial bonds is easier described by the VSEPR theory.

In total, the usefulness of the concept of hypervalency is questionable, since the bonding in hypervalent molecules does not differ from that in Lewis octet molecules, except that the coordination numbers may be higher due to the increased size of post second-row elements. Still many questions remain. Especially in the case of sulfur and phosphor compounds with a valency of four, which violate the octet rule by the formation of $\mathrm{P}=\mathrm{O}$ or $\mathrm{S}=\mathrm{O}$ double bonds in the Lewis notation, the involved orbitals and the exact distribution of the electrons in these bonds is discussed controversely. ${ }^{[185]}$ While both independent QTAIM and ELF studies have been performed on these compounds, ${ }^{[186,187]}$ their combined power has never been exhausted. The synergistic effects between ELF/ELI can be clarified when keeping in mind 
the strengths of each method. QTAIM partitions the molecular electron density into atomic densities. On integration, atomic charges can be obtained easily. Unfortunately it exists no analog basin for bonding electrons, over which could be integrated to deduce the bond order. Bonds between a pair of atoms are mainly characterized by properties of the bond critical point (BCP) between them. In contrast, the ELI partitions the space into electron localization domains, which in the case of closed-shell systems correspond to the classical electron pairs of the Lewis formulas. While monosynaptic ELI basins are associated with lone par regions in a molecule, disynaptic ELI basins are an indicator for a classical 2-electron-2-center bond. By integrating the electron population of a disynaptic bonding basin $\bar{M}[\boldsymbol{C}, \boldsymbol{Y} \mathbf{]}$, the bond order $b$ between two atoms $X$ and $Y$ can be deduced from the average electron pair number (Eq. 5-1). ${ }^{[188]}$

$$
b_{X Y}=\frac{\bar{N}[V(X, Y)]}{2}
$$

In 2001 Jansen et al. published a method to obtain a quantitative measure of bond polarity by evaluation of the partitioning of $\bar{F}\left[\boldsymbol{K}, Y \mathbf{]}\right.$ between the atomic QTAIM basins. ${ }^{[189]}$ This is done by an intersection of the disynaptic ELI basin $\bar{M} \mathbf{L} / \boldsymbol{C}, Y \mathbf{]}$ with the QTAIM atoms $X$ and $Y$ and denoted by $\bar{M}[V W, Y X]$ and $\bar{M} V \boldsymbol{V}, Y \mathbf{Y} Y \mathbf{1}$. As a result it can be stated how much electrons a given element contributes to the chemical bond. This allows the definition of the bond polarity index, which is given by

$$
p_{n Y}=\frac{a_{X}-a_{Y}}{100}=\frac{\bar{M}[V(X, Y) \mid X]-\bar{M}[V(X, Y, Y]}{\bar{M}[V(X, Y) \mid X]+\bar{M}[V(X, Y Y Y]}
$$

Hereby ax should be the larger percentual value. Values for $p_{X Y}$ range between 0 for perfect covalent bonding and 1 for a total ionic bond. The advantage of this approach is that no reference to one of the available atom or group electronegative scales is needed to determine the polarity of a chemical bond.

For this reason this methodology was applied in the following work to the neutral compounds $\mathrm{S}(\mathrm{NH})_{3}, \mathrm{H}_{3} \mathrm{PO}$ and to the anionic $\mathrm{SO}_{4}{ }^{2-}$ and $\mathrm{PO}_{4}{ }^{3-}$. These four molecules are hypervalent on the assumption of $\mathrm{S}=\mathrm{N}$ and $\mathrm{P}=\mathrm{O}$ double bonds to be present, while a formulation of these bonds as single bonds including formal charges $\left(\mathrm{S}^{+}-\mathrm{N}^{-}\right.$or $\left.\mathrm{P}^{+}-\mathrm{O}^{-}\right)$ preserves the electron octet at the central atom. The quantitative estimation of the bond polarity in combination with the bond order according to the combined QTAIM/ELI approach should help to distinguish between these extreme cases and to analyze the electron distribution in these bonds. 


\subsection{Triimid}

Sulfur triimides are in the focus of our working group both synthetically and analytically for a fairly long time. First synthesized by Glemser and Wegener in 1970 by reaction of sulfurnitridetrifluoride with bis( $N$-trimethylsilylimidosulfurdifluoride $),{ }^{[190]}$ the application of these compounds suffered a long time from limited synthetic access. An alternative route to sulfurtriimides proceeding over the oxidation of $\left[\mathrm{Li}_{4}\left\{(\mathrm{NtBu})_{3} \mathrm{~S}\right\}_{2}\right]$ has presented by Fleischer et al.. ${ }^{[191]}$

In these compounds, which are isolobale to $\mathrm{SO}_{3}$, sulfur has a +6 oxidation state. In Lewis notion bonding to the three nitrogen atoms could be achieved by the formation of three double bonds. This picture is supported by the planarity of the $\mathrm{SN}_{3}$ moiety in combination with short sulfur-nitrogen distances of around $150 \mathrm{pm} .{ }^{[192]}$ The possibility to form six bonds in total was explained by a valence expansion on sulfur, where d-functions in the role of true valence orbitals (in contrast to mere polarization functions) are involved. [193] This obviously violates the octet rule and leads to a so called hypervalent sulfur atom. To elucidate the bonding in these compounds, our working group performed both experimental and theoretical charge density studies. D. Leusser analyzed the electron density (ED) distribution of $\mathrm{S}\left(\mathrm{N}^{t} \mathrm{Bu}\right)_{3}$ obtained from high-resolution X-ray data. ${ }^{[194]}$ From the occurrence of two valence shell charge concentrations (VSCCs) close to nitrogen, which can be interpreted as lone pair densities, he concluded this atom to be $\mathrm{sp}^{3}$-hybridized. On the other hand indicators like the ellipticity at the BCP implied a considerable degree of $\pi$-character for the $\mathrm{S}-\mathrm{N}$ bond. Theoretical results accomplished by J. Henn were not able to reproduce these two VSCCs close to nitrogen. Only one VSCC lying in the $\mathrm{SN}_{3}$ plane could be found, which is indicative for a $\mathrm{sp}^{2}$ hybridization on nitrogen. This is in agreement with the existence of a sulfurnitrogen double bond, which also could be anticipated from the planarity of the $\mathrm{SN}_{3}$ moiety. Overall both theoretical and computational results agree in a strong polarity of the $\mathrm{S}-\mathrm{N}$ bond with a $\pi$-system above and below the $\mathrm{SN}_{3}$ plane. Leusser et al. concluded that the situation in sulfur triimide can be described best as a 4-center-6-electron bond with the $\pi$-orbitals being strongly polarized. Therefore ionic resonance structures are allowed to contribute.

To quantify the number of electrons who participate in $\mathrm{S}-\mathrm{N}$ bonding and to determine the polarity of the bond, theoretical calculations on the B3-LYP/cc-pVTZ level were performed and analyzed with the new combined QTAIM/ELI approach. The topological analysis yielded bond critical points between the sulfur and nitrogen atoms with $\rho\left(\mathbf{r}_{\mathrm{BCP}}\right)$ of $1.93 \AA^{-3}$ and $\nabla^{2} \rho\left(\mathbf{r}_{\mathrm{BCP}}\right)-2.00 \AA^{-5}$. Although the absolute value of $\nabla^{2} \rho\left(\mathbf{r}_{\mathrm{BCP}}\right)$ is very small, the negative sign indicates a slight accumulation of charge density in the bonding region, emphasizing the covalent amount in the polar $\mathrm{S}-\mathrm{N}$ bond. 

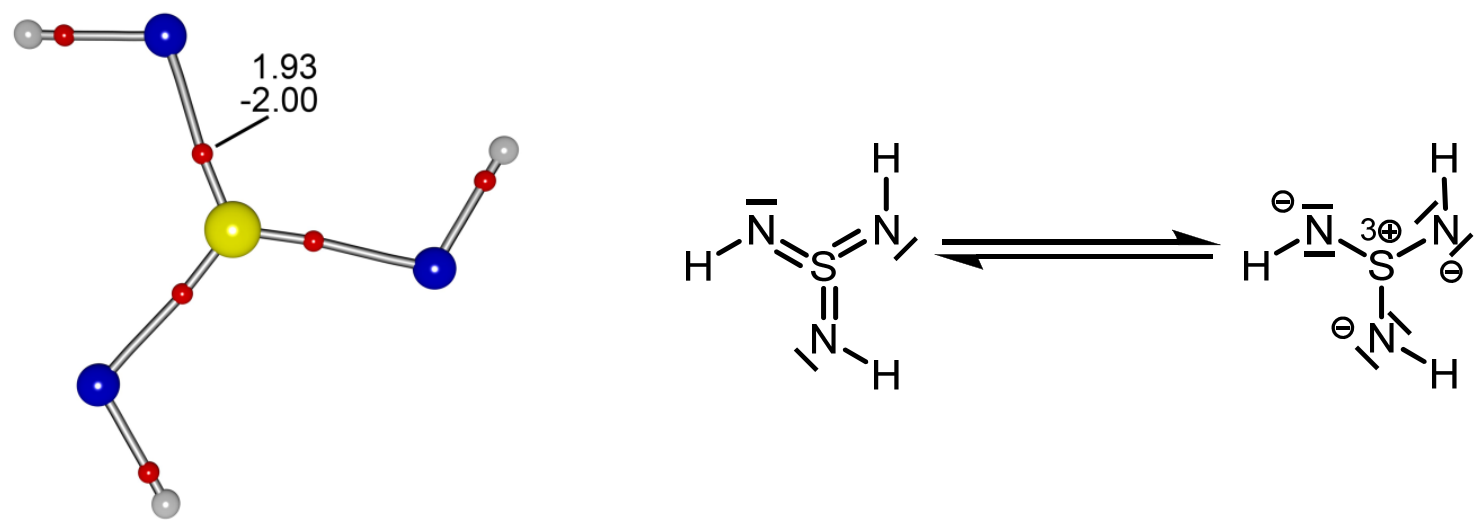

Figure 5-5. Left: Molecular graph of 10. The small red spheres indicate BCPs, with $\rho\left(\mathbf{r}_{\mathrm{BCP}}\right)\left[\mathrm{e} \AA^{-3}\right]$ (upper number) and $\nabla^{2} \rho\left(\mathrm{r}_{\mathrm{BCP}}\right)\left[\mathrm{e} \AA^{-5}\right]$ (bottom number). Right: Two Lewis resonance structures for $\mathrm{S}(\mathrm{NH})_{3}$.

After calculating the QTAIM and ELI grids (mesh size 0.05 Bohr) with DGRID-4.4, these data can also be visualized in form of isosurfaces (figure 5-6). It can be shown that the qualitative results of both methods draw the same picture. At a level of -1.20 e $\AA^{-5}$ for QTAIM and 1.2 for ELI, a whole in the $\mathrm{SN}_{3}$ plane at the bisection of the $\mathrm{N}-\mathrm{S}-\mathrm{N}$ angles can be seen. In QTAIM this hole corresponds to a region of charge depletion. Consequently, the ELI predicts at the same space region low values for electron localization. This hole has been interpreted as the preferred site of attack for nucleophiles, explaining why only small organolithium compounds (MeLi, PhLi) react with sulfur triimides, ${ }^{[191]}$ while no addition can be observed in the case of the bulky $t$ BuLi. ${ }^{[195]}$ If attack would take place from above or below the $\mathrm{SN}_{3}$ plane, this behavior could not be explained.
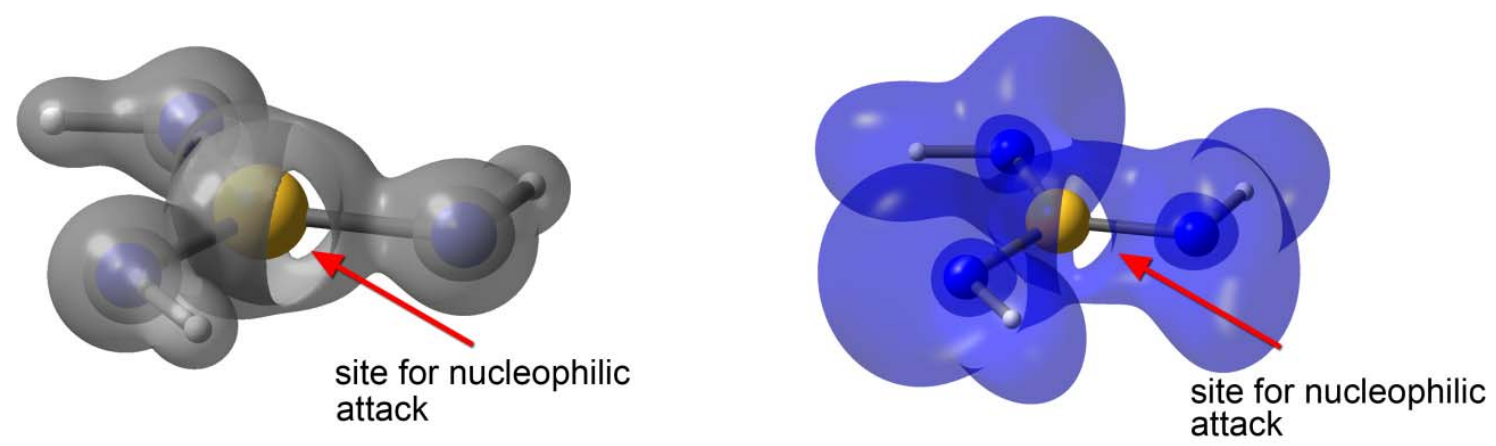

Figure 5-6. Isosurface representation of $\nabla^{2} \rho$ on a level of -1.20 e $\AA^{-5}$ (left) and ELI at a level of 1.2 (right) for 10.

By raising both the QTAIM and the ELI level to $-9.64 \mathrm{e} \AA^{-5}$ and 1.4 respectively, bonding features become visible (figure 5-7). The pictures obtained still yield qualitative results, but a distinct $\pi$-character of the S-N bond can be anticipated from high QTAIM/ELI values above 
and below the $\mathrm{SN}_{3}$ plane. Furthermore, QTAIM reveals an area of charge depletion around the sulfur atom, and the electron density is polarized towards the nitrogen atom. In the ELI isosurface representation features that resemble the nitrogen lone pairs can be detected also.
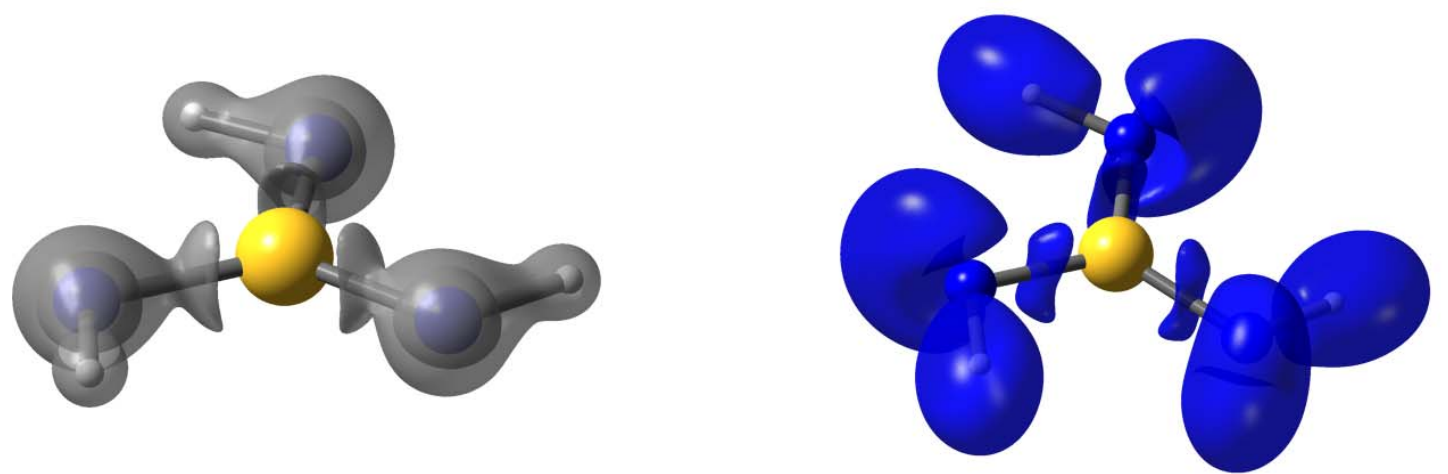

Figure 5-7. Isosurface representation of $\nabla^{2} \rho$ on a level of $-9.64 \mathrm{e} \AA^{-5}$ (left) and ELI at a level of 1.4 (right) for 10.

To get a quantitative picture of the bond, the atomic charges over the QTAIM basins were integrated. The sulfur atom bears a charge of +2.76 e and each nitrogen atom a charge of 1.36 e. While these give a first hint of the polarity, QTAIM charges generally suffer to be too large. In addition, it is not possible to deduce a bond polarity from atomic charges alone. ${ }^{[189]}$ Therefore, in the next step the disynaptic ELI basins between sulfur and nitrogen have been envisaged (scheme 5-1). Two of these can be identified, both with their ELI maximum not on the direct atomic interaction line, but above and below the $\mathrm{SN}_{3}$ plane. The deviation from a rotational symmetry around the atomic interaction line (AIL) is reflected by the eccentricity of 0.53 of the ELI basins too. Surprisingly, the integration of the two disynaptic basins yielded only a total electron population of 2.42 electrons. Consequently, the bond order is 1.21 and closer to a single than to a double bond. Thus, while the number and location of these basins could have been interpreted as a double bond, taking the determined bond order into account this interaction could be classified as a single bond which electron distribution bears resemblance to a double bond. Finally, the intersection of the QTAIM and ELI basins shows, that sulfur offers 1.02 and nitrogen 1.42 electrons for bonding. The calculated bond polarity index is 0.17 , which is in compliance to a predominantly covalent character with small ionic contributions. 


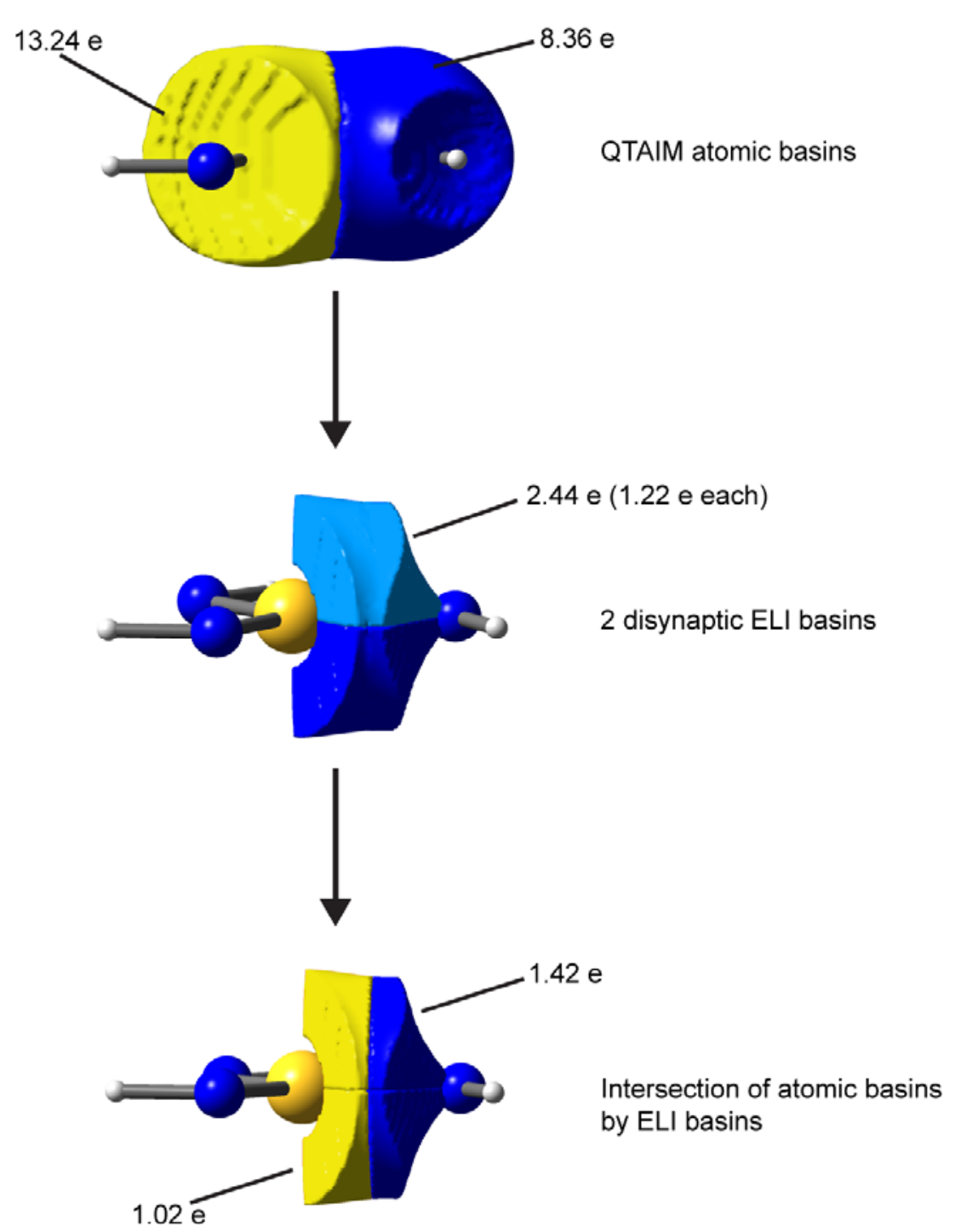

Scheme 5-1. Atomic basins of sulfur and nitrogen in 10, which are intersected by the disynaptic ELI basins.

The determination of the electron population of the monosynaptic valence basins on nitrogen yields 3.54 electrons in total (figure 5-8). This is not far away from two electron pairs wherefore the hybridization on nitrogen seems definitely to have more $\mathrm{sp}^{3}$ - than $\mathrm{sp}^{2}$ character. In contrast to QTAIM, where the determination of the hybridization is an "all or nothing" decision, as it is deduced from the number of VSCCs, integration over the monosynaptic ELI basins yields electron populations, which can also reflect intermediate cases between $\mathrm{sp}^{3}$ and $\mathrm{sp}^{2}$ hybridization. In total, the bonding in $\mathrm{S}(\mathrm{NH})_{3}$ can be described as a mainly polar covalent single bond which is reinforced by substantial m-back-bonding, resulting in a charge accumulation in the bonding region above and below the $\mathrm{SN}_{3}$ plane. 


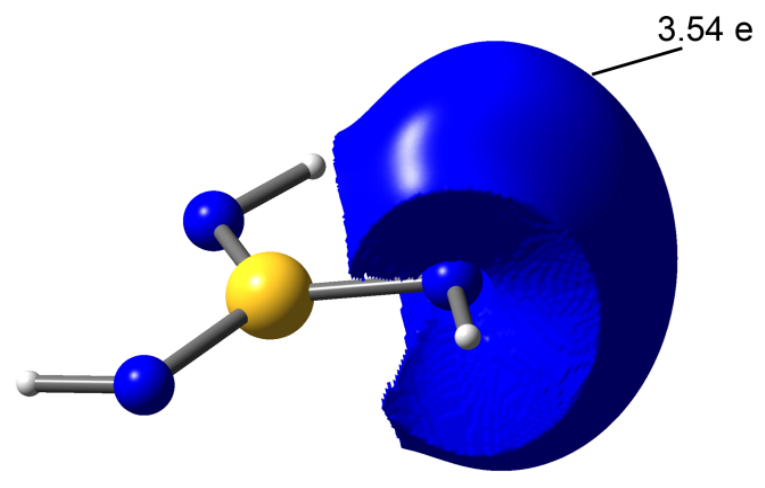

Figure 5-8. ELI isosurface representation of the monosynaptic nitrogen basin in $\mathbf{1 0}$.

\section{$5.3 \mathrm{H}_{3} \mathrm{PO}$}

The second example covers he phosphor oxygen double bond on the basis of $\mathrm{H}_{3} \mathrm{PO}$. The $\mathrm{P}=\mathrm{O}$ bond has been discussed controversially in the literature. Short bond distances as well as the strength of this bond have been regarded as an indicator for a double bond. As $d-$ orbital participation had to be excluded because their energy is too high, ${ }^{[182]}$ a different explanation for the phosphor-oxygen bond was needed. Basically, three different bonding schemes have been postulated (figure 5-9). ${ }^{[185]}$ The first (a) describes the bond as being composed of a $\sigma$-donor bond from the phosphor lone pair to oxygen. To allow back-bonding, the empty d-orbitals have been replaced by a set of suitable acceptor orbitals of e-symmetry of the $\mathrm{R}_{3} \mathrm{P}$ moiety. This back-bonding has been also described as negative hyperconjugation. However, in this description the PO bond is actually a triple bond, since two occupied porbitals on oxygen are involved in $\pi$-back-bonding. Model b goes even one step further, since in this case oxygen is $\mathrm{sp}^{3}$ hybridized and in addition to one $\sigma$-bond there are three $\pi$ back-bonds. Hereby, the oxygen lone pairs would occupy a staggered conformation with respect to the substituents on phosphor. The third model (c) consists of the concept of banana bonds. This view differs from $\mathbf{a}$ and $\mathbf{b}$ in that no separation in $\sigma$ and $\pi$ bonds is present but the triple bond is composed of three equivalent bent bonds.

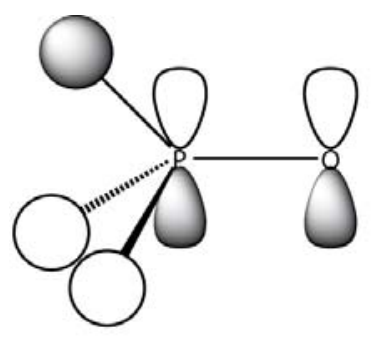

a

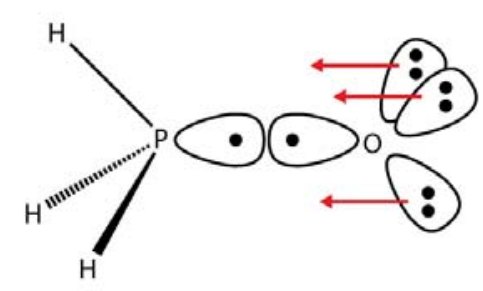

b

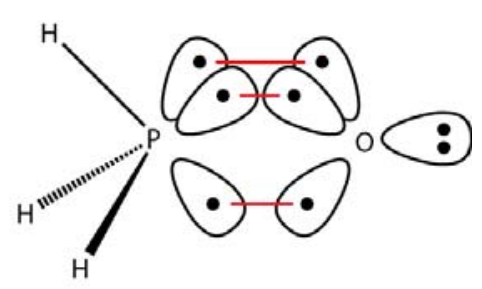

c

Figure 5-9. Three different descriptions of the P-O bond. ${ }^{[186]}$ 
In 1998 Sundberg et al. revised the bonding situation of these compounds by using the QTAIM theory. ${ }^{[186]}$ By evaluating the Laplacian around oxygen in $\mathrm{H}_{3} \mathrm{PO}$, they detected three VSCCs which were staggered relative to the $\mathrm{P}-\mathrm{H}$ bonds. On the AIL only one VSCC was found, which is very close to the oxygen. In contrast, no corresponding VSCC was found in the atomic basin of phosphor. These facts confirm the assertion, that the $\mathrm{P}-\mathrm{O}$ bond is a highly polar $\sigma$-bond which substantial ionic contributions.

Although phosphor is positively charged, in the QTAIM investigations a m-interaction with the three oxygen lone pairs can neither be approved nor excluded. Therefore the combined QTAIM/ELI method was applied (B3-LYP/TZVPP level of theory) to $\mathrm{H}_{3} \mathrm{PO}$ to review whether a more precise description of the $\mathrm{P}-\mathrm{O}$ bond is possible.

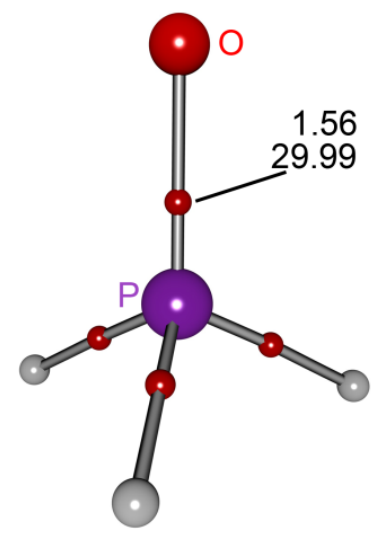<smiles>COP</smiles>

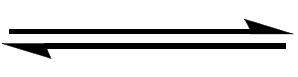<smiles>[O-][PH3+]</smiles>

Figure 5-10. Left: Molecular graph of 11. The small red spheres indicate BCPs, with $\rho\left(\mathbf{r}_{\mathrm{BCP}}\right)\left[\mathrm{e} \AA^{-3}\right]$ (upper number) and $\nabla^{2} \rho\left(\mathbf{r}_{\mathrm{BCP}}\right)\left[\mathrm{e} \AA^{-5}\right]$ (bottom number). Right: Two Lewis resonance structures for $\mathrm{H}_{3} \mathrm{PO}$.

A dramatic difference to the $\mathrm{S}-\mathrm{N}$ bond in 10 is obvious form a value of $29.99 \mathrm{e}^{-5}$ for $\nabla^{2} \rho\left(\mathbf{r}_{\mathrm{BCP}}\right)$. While in the polar $\mathrm{S}-\mathrm{N}$ bond of the sulfur triimide the covalent character prevailed, in this case a substantial ionic contribution is present. By looking at the isosurface plots for QTAIM (-9.64 e $\AA^{-5}$ ) and ELI (level 1.4) a second remarkable distinction to the S-N bond can be seen. While there a $\pi$-type interaction could be identified at first sight, in the present case the electrons seem to be accumulated in a rotational symmetric way around the phosphoroxygen trajectory (figure 5-11). 

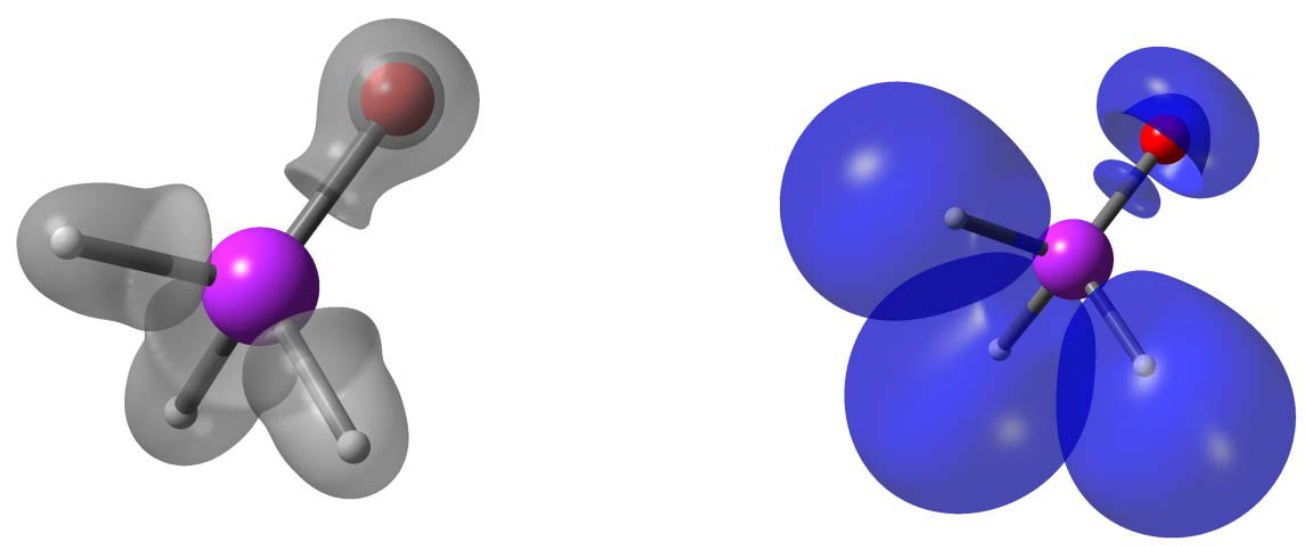

Figure 5-11. Isosurface representation of $\nabla^{2} \rho$ on a level of $-9.64 \mathrm{e} \AA^{-5}$ (left) and ELI at a level of 1.4 for 11.

Indeed, the ELI reveals only one disynaptic basin with its maximum on the AlL. This basin contains 1.95 electrons, which yields a bond order of 0.98 . This is in compliance with one single $\sigma$-bond. By intersecting the disynaptic ELI basin with the QTAIM basin of phosphor and oxygen, it can be seen how the bonding electrons are apportioned between the respective atoms. Only 0.32 of the 1.95 electrons of the bonding basin are located in the phosphor atomic basin, while 1.63 electrons are located in the oxygen atomic basin. This illustrates an enormous charge transfer from phosphor to oxygen during the formation of the $\sigma$-bond. This also shows up in the atomic partial charges, which are +2.93 e for phosphor and -1.22 e for oxygen. The derived bonding polarity index is 0.67 , thus, in contrast to the sulfur triimide, the $\mathrm{P}-\mathrm{O}$ bond has a pronounced ionic character. 


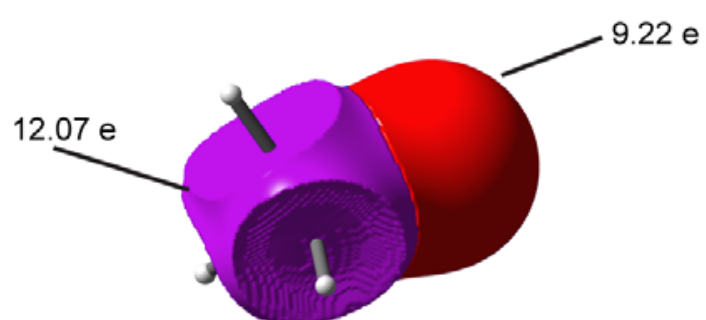

QTAIM atomic basins

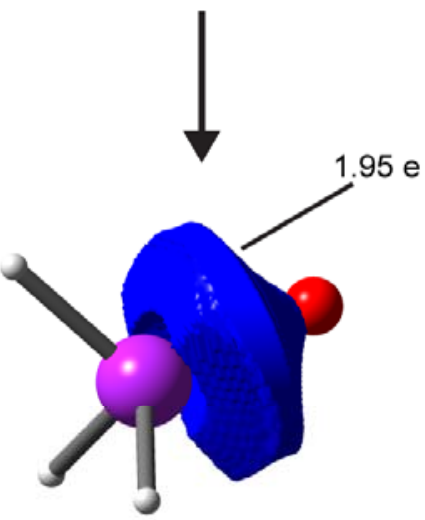

disynaptic ELI basin
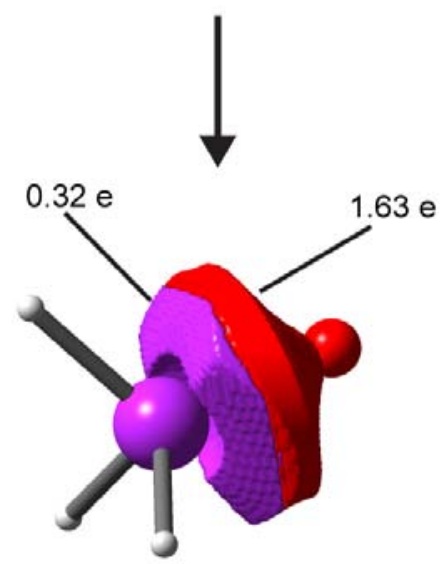

Intersection of atomic basins by ELI basin

Scheme 5-2. Atomic basins of sulfur and nitrogen in 11, which are intersected by the disynaptic ELI basins

Integration over the monosynaptic lone pair basins on oxygen yields 5.46 electrons in total (figure 5-12). The basins could not be separated in three distinct localization domains, but the average electron number corresponds to three lone pairs, so the existence of a double bond between phosphor and oxygen can be excluded. Whether the deviation from six electrons can be attributed to back-bonding is questionable, since this should raise the electron density in the bonding region. This can be observed in $\mathrm{S}(\mathrm{NH})_{3}$ where the $\mathrm{S}^{+}-\mathrm{N}^{-} \sigma-$ bond is supported by $\pi$-interactions raising the bond order to 1.2. A similar effect seems not to occur for the $\mathrm{P}-\mathrm{O}$ bond, where the bond order is only 0.98 . 


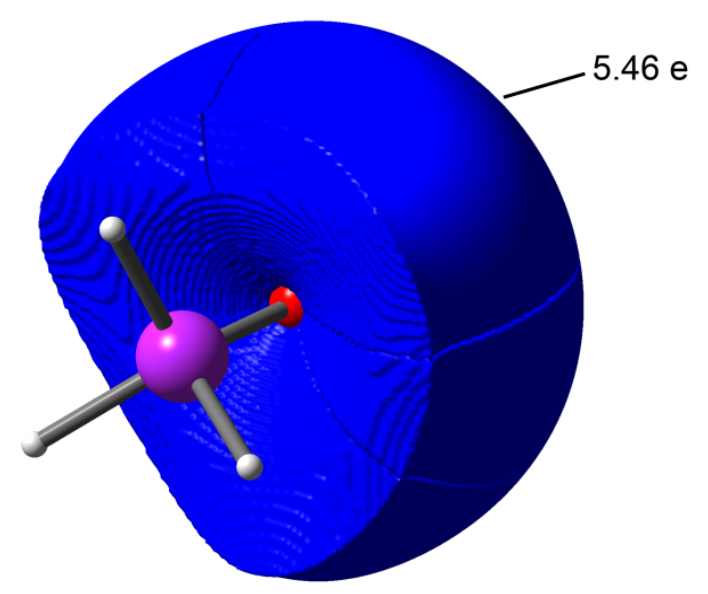

Figure 5-12. ELI isosurface representation of the monosynaptic nitrogen basin in $\mathbf{1 1 .}$

\section{$5.4 \mathrm{SO}_{4}{ }^{2-}$}

The next two molecules under investigation are the sulfate anion and the phosphate anion. The structures were optimized on the B3-LYP level of theory and their sulfur- and phosphor-oxygen bonds will be compared to the $\mathrm{S}-\mathrm{N}$ and $\mathrm{P}-\mathrm{O}$ bonds in 10 and 11 to examine the effect of negative charges on oxygen to the m-backbonding capabilities of the lone pairs. Generally, the hypervalent notation of the sulfate anion is given preference by most textbooks of inorganic chemistry. Since all S-O bonds are equal, resonance structures are needed, which superposition constitutes the real bonding situation. The notation of the sulfate anion with four sulfur-oxygen single bonds including partial charges is still considered unfamiliar by many chemists.
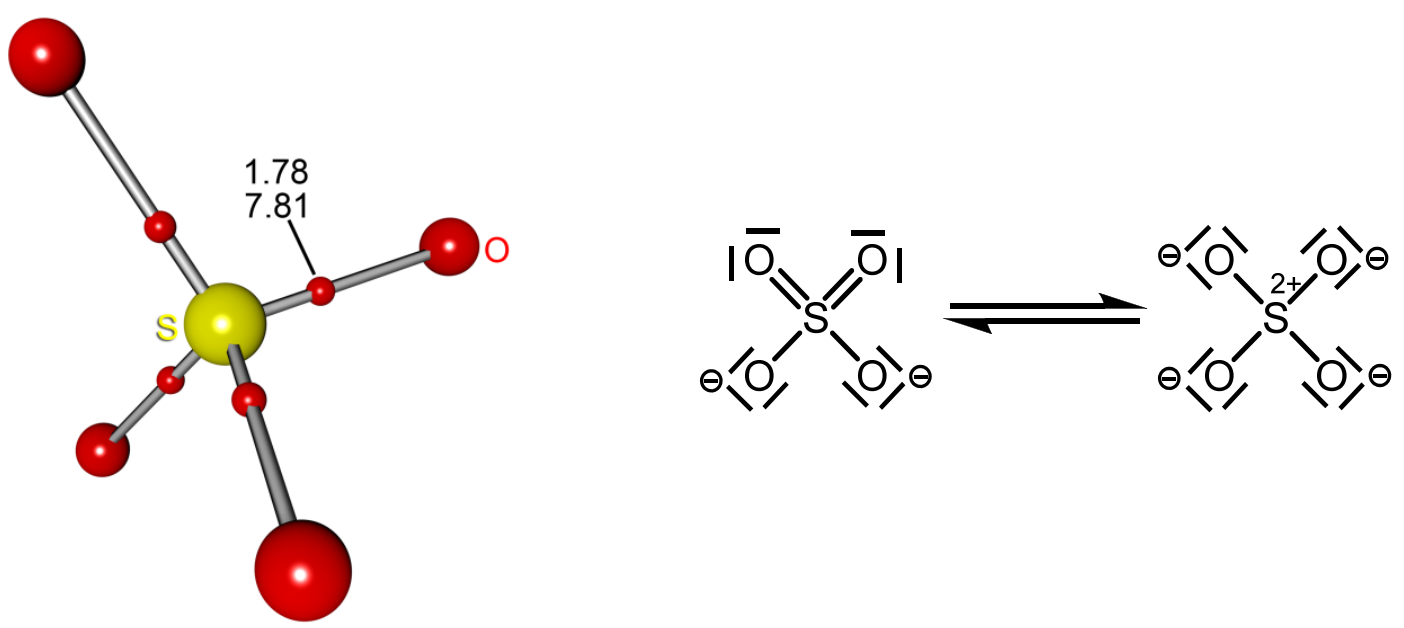

Figure 5-13. Left: Molecular graph of 12. The small red spheres indicate BCPs, with $\rho\left(\mathbf{r}_{\mathrm{BCP}}\right)\left[\mathrm{e} \AA^{-3}\right]$ (upper number) and $\nabla^{2} \rho\left(\mathbf{r}_{\mathrm{BCP}}\right)\left[\mathrm{e} \AA^{-5}\right]$ (bottom number). Right: Two Lewis resonance structures for the sulfate anion. 
By having a first look at the BCP a positive Laplacian of 7.81 e $\AA^{-5}$ seen. Although this is already a hint for a polar bond, it gives no quantitative measure of the bond order or polarity.

Examining the Laplacian isosurface on a level of $-9.64 \mathrm{e}^{-5}$ shows a charge accumulation polarized towards the oxygen atoms, while the region around the sulfur atom is electronically depleted (figure 5-14). On an ELI level of 1.4, localization of electrons can be observed in the valence shells behind the oxygen atoms, which have a torus like shape and correspond to the oxygen lone pairs. In addition electrons are localized between the sulfur and the oxygen atoms with a rotational symmetry around the AIL.
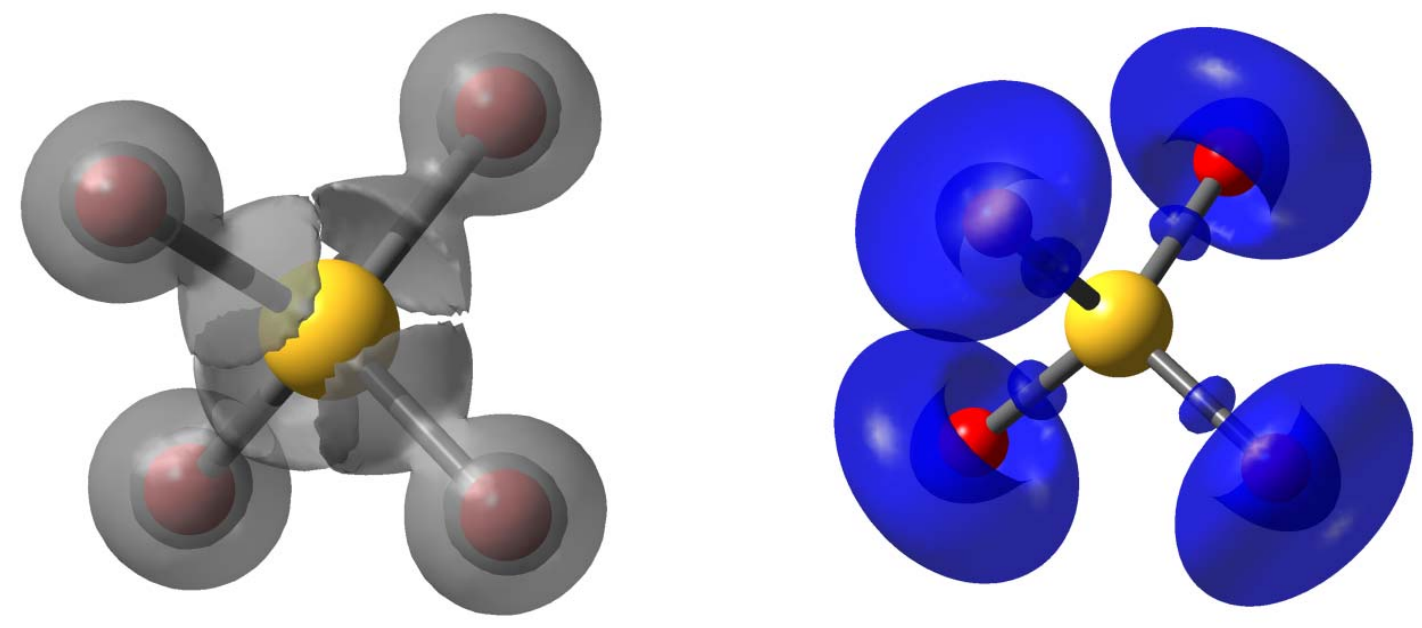

Figure 5-14. Isosurface representation of $\nabla^{2} \rho$ on a level of $-9.64 \mathrm{e} \AA^{-5}$ (left) and ELI at a level of 1.4 for 12.

The sulfur atom features a positive charge of $+3.37 \mathrm{e}$, which is substantially higher than in the triimide 10. The positive charge is balanced by a negative charge of $-1.37 \mathrm{e}$ at each oxygen atom. Although there are already two negative charges in the $\mathrm{SO}_{4}{ }^{2-}$ anion located on four oxygen atoms, leading formally to a charge of -0.5 e on each, still electron density is drawn away from the sulfur atom. In the ELI, between sulfur and all oxygen atoms, disynaptic basins with populations of 1.62 e are found (scheme 5-3). Hence, the resulting bond order $(0.81)$ is definitely below a single bond. It has to be noted that both the eccentricity of the disynaptic ELI basins as well at the ellepticities at the BCPs are zero. Despite the high positive charge of the sulfur atom, no back-bonding of the oxygen lone pairs can be detected.

As anticipated, the bonding electrons are not shared equally between both elements. Only 0.6 e reside in the atomic basin of sulfur, while $1.02 \mathrm{e}$ are in that of oxygen. This results in a bond polarity of 0.26 . While each bond is just slightly more polar than in 10 , the higher charge of sulfur originates in the higher valency of four. 

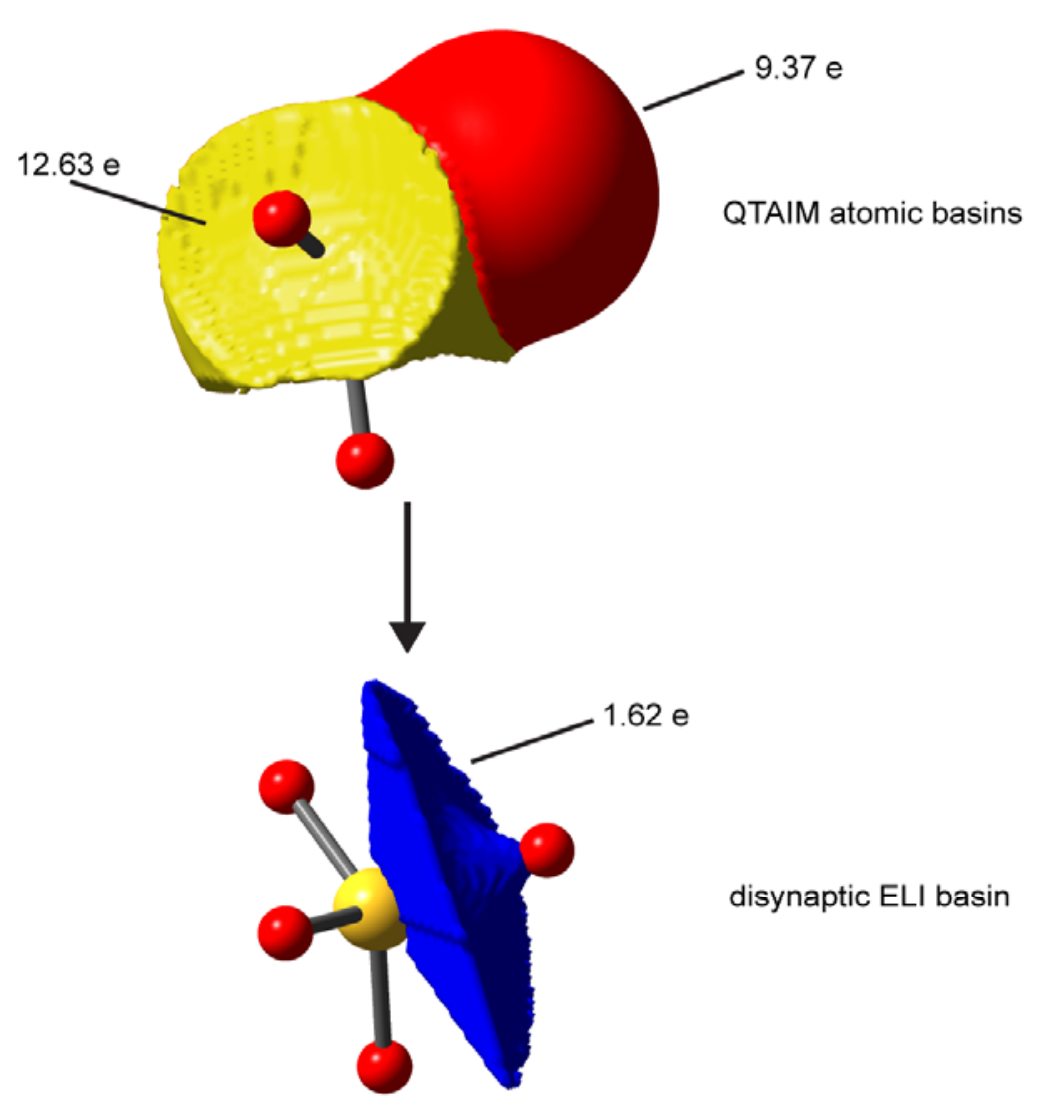

disynaptic ELI basin

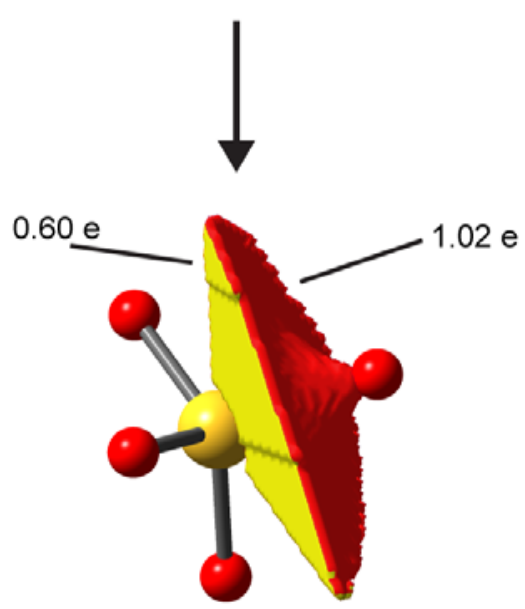

Intersection of atomic basins by ELI basin

Scheme 5-3. Atomic basins of sulfur and nitrogen in 12, which are intersected by the disynaptic ELI basins.

In 12 three separated monosynaptic basins on oxygen were detected, which have a staggered conformation with respect to the other sulfur-oxygen bonds (figure 5-15). 6.24 electrons occupy these localization domains, which is indicative of three full lone pairs on oxygen organized in $\mathrm{sp}^{3}$ hybrid orbitals. 

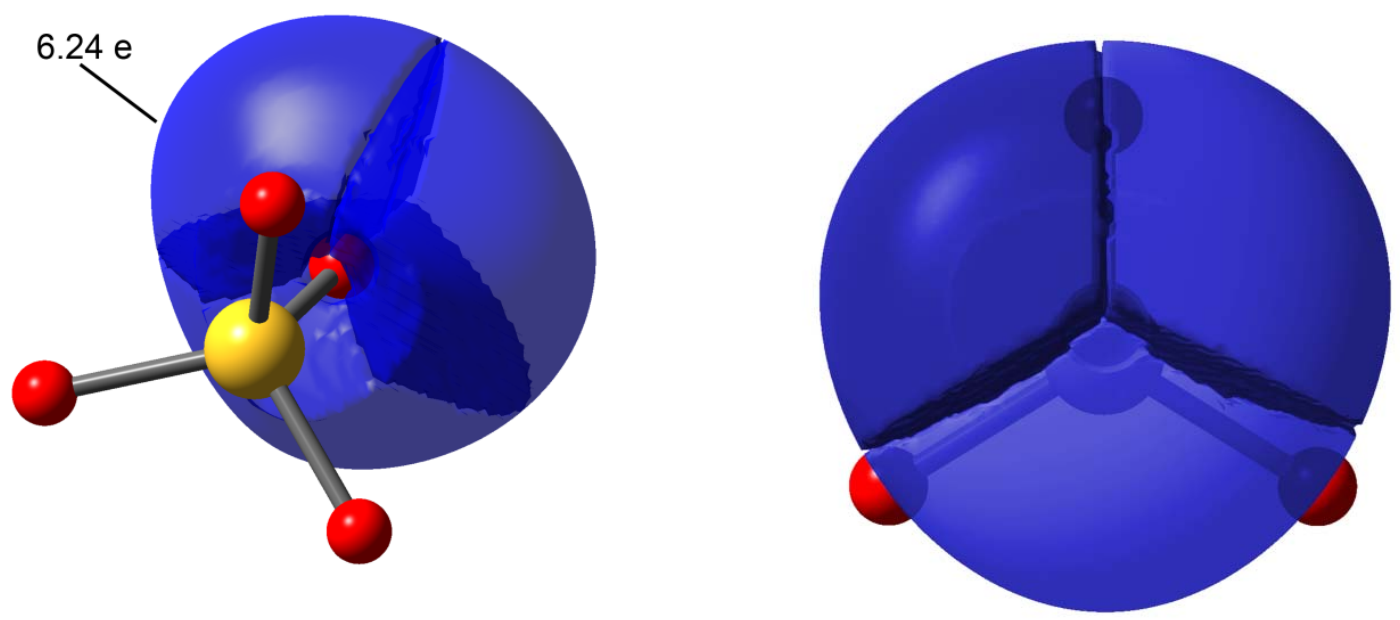

Figure 5-15. ELI isosurface representation of the monosynaptic oxygen basins in $\mathbf{1 2 .}$

\section{$5.5 \mathrm{PO}_{4}^{3-}$}

The last example considers the phosphate anion. Comparing values at $\rho\left(\mathbf{r}_{\mathrm{BCP}}\right)$ of the phosphor-oxygen with the sulfur-oxygen bond in 12 , a reduction from 1.78 to $1.23 \mathrm{e} \AA^{-3}$ can be observed. Concomitantly, $\nabla^{2} \rho\left(\mathbf{r}_{\mathrm{BCP}}\right)$ raises from 7.81 to 16.20 e $\AA^{-5}$. Thus, the P-O bond seems to be more ionic than the S-O bond.
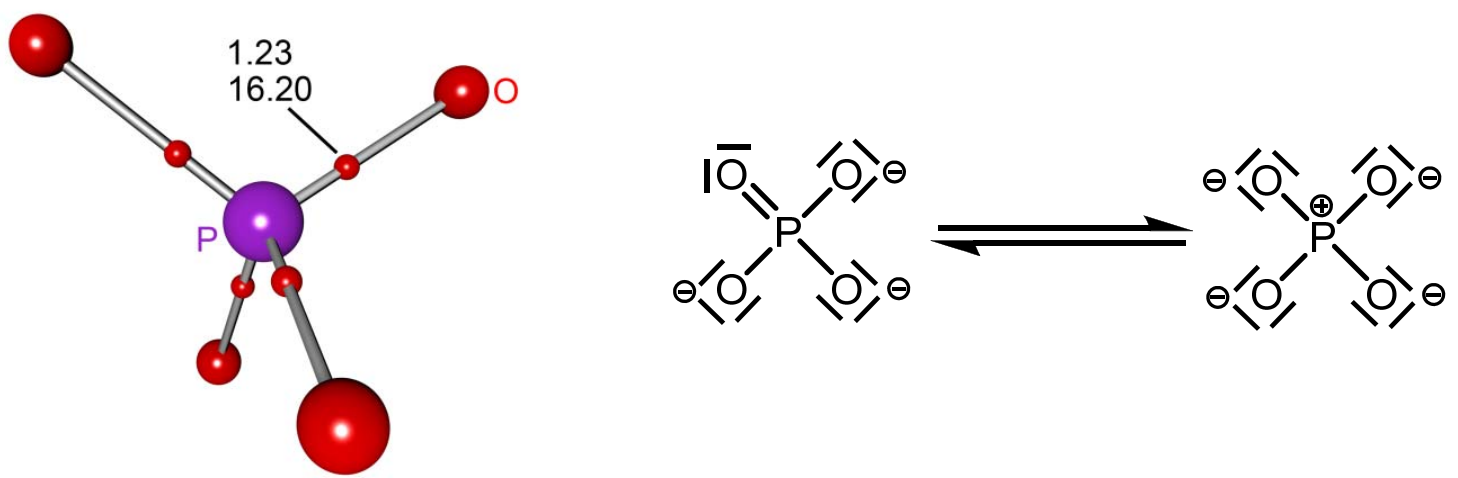

Figure 5-16. Left: Molecular graph of 13. The small red spheres indicate BCPs, with $\rho\left(r_{\mathrm{BCP}}\right)\left[\mathrm{e} \AA^{-3}\right]$ (upper number) and $\nabla^{2} \rho\left(\mathbf{r}_{\mathrm{BCP}}\right)\left[\mathrm{e} \AA^{-5}\right]$ (bottom number). Right: Two Lewis resonance structures for the phosphate anion.

Looking at the Laplacian isosurface, the situation can be visualized. The region of charge accumulation is further shifted away from the bonding region towards the oxygen atom. $A$ further increase in bond polarity would lead to a closed shell interaction. The ELI yields a complementing picture and the disynaptic electron localization domain is very close to oxygen (figure 5-17). 

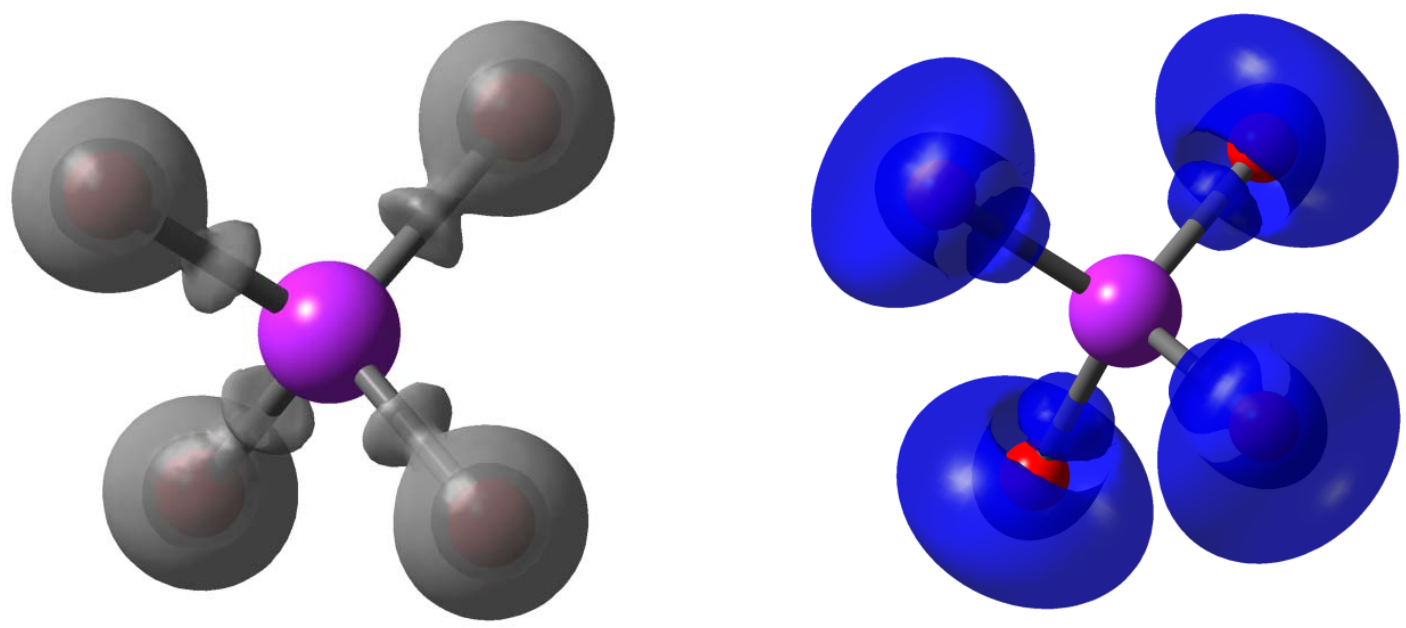

Figure 5-17. Isosurface representation of $\nabla^{2} \rho$ on a level of $-9.64 \mathrm{e} \AA^{-5}$ (left) and ELI at a level of 1.4 for 13.

Accordingly, the integrated charge of oxygen $(-1.59 \mathrm{e})$ is higher than in the sulfate anion $12(1.37 \mathrm{e})$. The charge at phosphor is $+3.33 \mathrm{e}$. The disynaptic phosphor-oxygen bonding basin contains only 1.55 e (scheme $5-4$ ). This results in a bond order of 0.78 , which is comparable to that in 12 (0.81). However, by intersecting the disynaptic ELI basin with the atomic QTAIM basins, it can be seen, that the 1.55 bonding electrons are mainly located in the atomic basin of oxygen (1.18 e), and only 0.37 electrons remain in the atomic basin of phosphor (scheme 5-4). Thus, a substantial charge transfer from phosphor to oxygen has taken place. Therefore, the polarity index of the $\mathrm{P}-\mathrm{O}$ bond is with 0.52 more than twice as large as for the $\mathrm{S}-\mathrm{O}$ bond in $\mathbf{1 2}$. Again, both eccentricity and ellepticity are zero and no $\pi-$ component in the $\mathrm{P}-\mathrm{O}$ bond can be detected. 

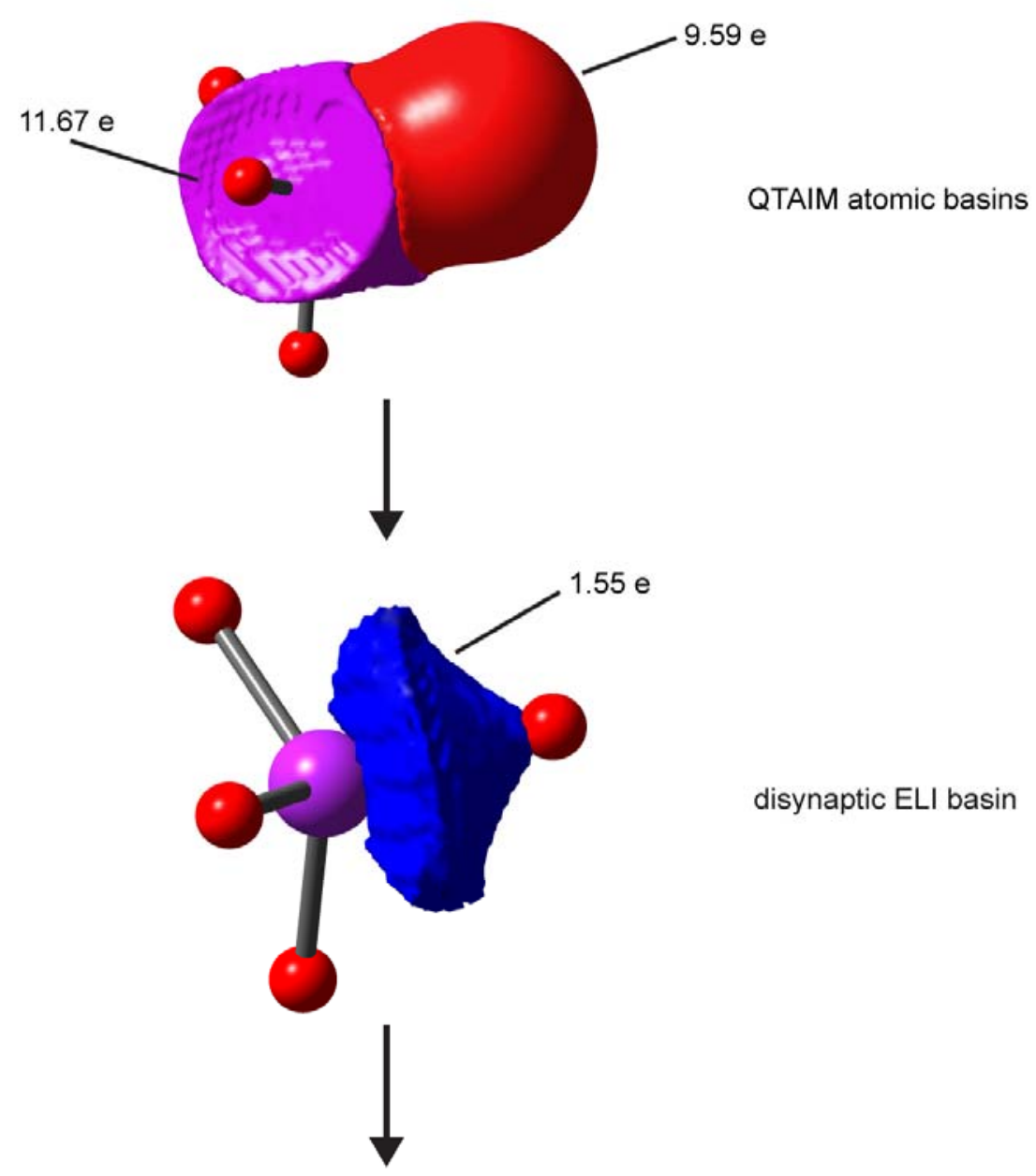

disynaptic ELI basin

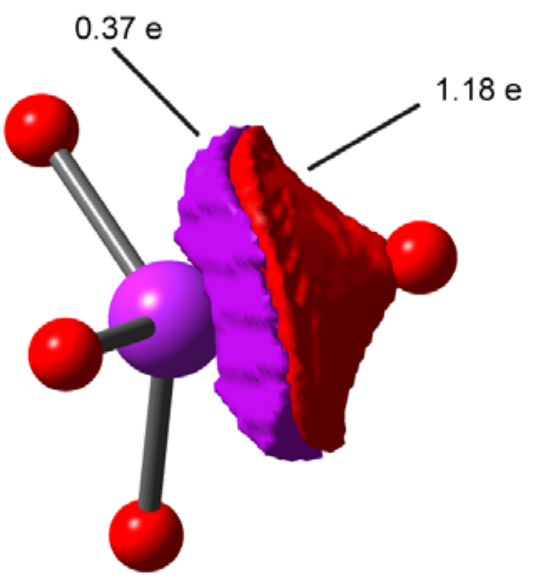

Intersection of atomic basins by ELI basin

Scheme 5-4. Atomic basins of sulfur and nitrogen in 13, which are intersected by the disynaptic ELI basins.

Finally, the electron population in the monosynaptic ELI basins behind the oxygen atom was analyzed. The number of electrons (6.29) in the three basins, which are again staggered with respect to the remaining $\mathrm{P}-\mathrm{O}$ bonds (figure 5-18), again suggests three lone pairs at the oxygen atom and confirms the low bond order of 0.78 . 

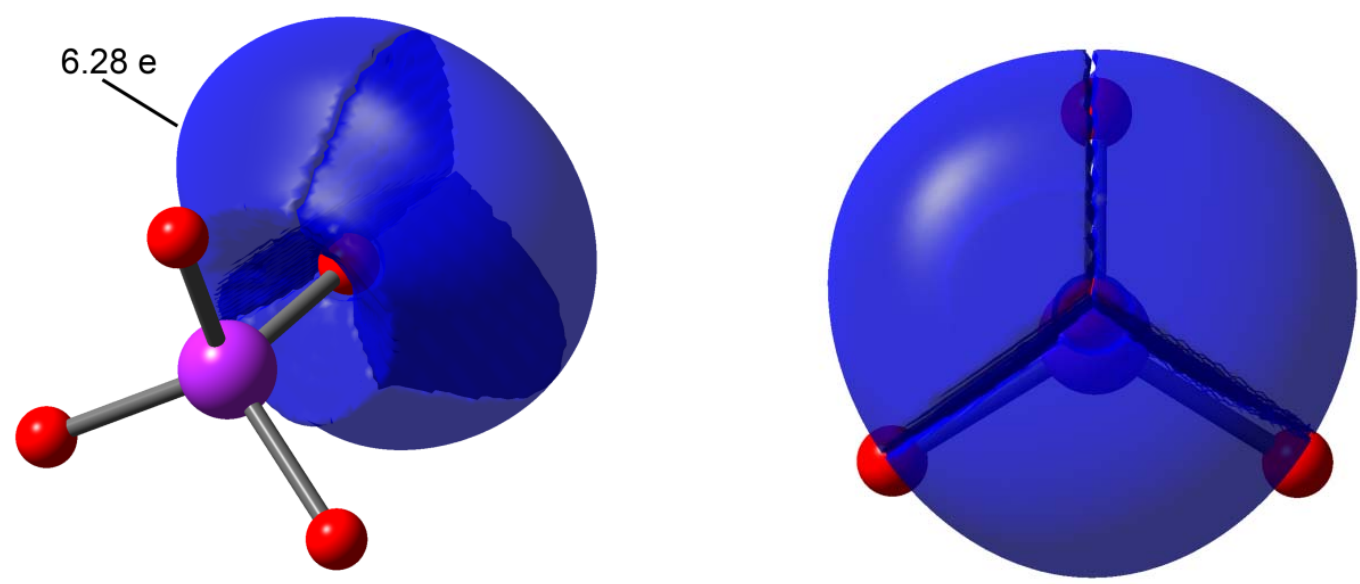

Figure 5-18. ELI isosurface representation of the monosynaptic oxygen basins in 13.

\subsection{Conclusion}

A combined QTAIM/ELI study was performed to study phosphor/sulfur-nitrogen/oxygen bonds in so called hypervalent molecules. To estimate the degree of $\pi$-bonding, electron population of ELI basins have been integrated to determine the bond order. Furthermore, the polarity of these bonds was described quantitatively by evaluating the distribution of the bonding electron numbers in the atomic QTAIM basins.

Only for the sufur triimide 10 a higher bond order than one was deduced. In this case $\pi-$ back-bonding is responsible for the accumulation of ED in the bonding region and its distribution above and below the $\mathrm{SN}_{3}$ plane. The back-bonding is probably possible because of the availability of an empty p-acceptor orbital on sulfur.

$\mathrm{H}_{3} \mathrm{PO}$ (11), $\mathrm{SO}_{4}{ }^{2-}(12)$ and $\mathrm{PO}_{4}{ }^{3-}(13)$ all have a higher valency than 10 . Therefore, the central atom is $s p^{3}$ hybridized and no empty $p$-orbital is available for $\pi$-bonding. This leads to the fact, that although the positive charges at the central atom are significantly higher than in 10, no back-bonding is observed, since the d-orbitals are ineglible as acceptor orbitals because of their energetic position. Furthermore, the bond order drops in 11 to 13 from 0.98 to 0.78 . Especially in the sulfate or phosphate anion it is not a question between single or double bond as appearing from the Lewis notion and certainly the description of the P-O interaction by triple bonds through negative hyperconjugation or banana bonds is highly questionable. Even the $\sigma$-bonds exhibit a mainly ionic character, thus not $\pi$-back-bonding but electrostatics plays a major role for the strengths and lengths of these bonds. 
The results can also be summarized in Lewis notion. Based on the computational studies it is possible to assign resonance structures which reflect the bonding situation within the limits of the Lewis model as close as possible. Accordingly, the sulfur triimide is best presented as a superposition of three resonance structures, which implies a bond order of 1.33 (figure 5-19). This is very close to the obtained ELI bond order of 1.21.

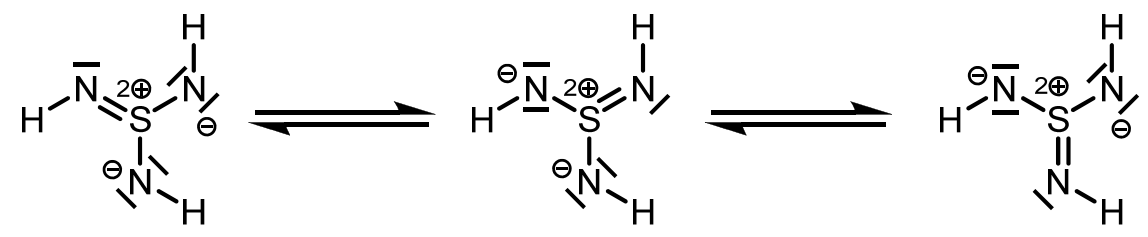

Figure 5-19. Most representative Lewis structures of 10.

For 11-13 it is not necessary to invoke more than one Lewis notion each. Their most appropriate Lewis structures are depicted in figure 5-20.<smiles>[O-][PH3+]</smiles><smiles>O=[PH]12(C=CO1)COC=CO2</smiles>

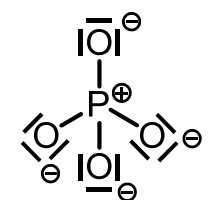

Figure 5-20. Most representative Lewis structures of 11, 12 and 13. 


\section{SUMmARY AND CONCLUSIONS}

The following thesis targeted two topics. The first one dealt with the synthesis and crystallization of reactive organometallic deprotonation agents and important metalated intermediates along the reaction pathway. Subsequently, the extremely air and moisture sensitive crystals were applied to an X-ray diffractometer at $-100^{\circ} \mathrm{C}$ using cryo technologies like the X-Temp 2 system. ${ }^{[11]}$ Thereby, the first crystal structure of a monomeric ortholithiated anisole was elucidated. This molecule is an important intermediate in the directed ortho metalation (DoM) and believed to be stabilized by an interaction between anisole's methoxy group and the adjacent lithium atom. In order to analyze and quantify this stabilizing effect, starting from the crystal geometry computational studies were performed. Two relatively new tools for bonding analysis, QTAIM and ELI, were applied and both coincidenced in that no attractive interactions between the lone pairs on the oxygen atom and the lithium cation can be detected. Thus, while lithium-oxygen interactions in a CIPE (complex induced proximity effect) precomplex are still discussed controversially, they don't seem to play a major role in the ortho-lithiated anisole.

The second compound under investigation was a mixed aggregate formed from the combination of methyllithium and lithium tert-butoxide. Complexes of this type particularly show a significantly increased reactivity compared to single organolithium reagents, a reason, why they were termed Lochman-Schlosser superbases. In the present case, it was shown that these reagents also exhibit oxygen scavenging capabilities. The determined crystal structure reveals a three-dimensional inverse crown ether, consisting of ten $t \mathrm{BuOLi}$ and four MeLi units, with two encapsulated $\mathrm{O}^{2-}$ anions. The coordination number of the oxo dianion and the types of interaction to the solvating lithium atoms were determined by theoretical QTAIM studies.

A further inverse crown ether structure was obtained by the addition of the diamine ligand tmeda to lithium diisoproplyamide (LDA). Lithium hydride, presumably formed by $\beta-\mathrm{H}$ elimination of one LDA unit, is solvated by excess LDA. Thereby, the hydride anion is captured in a pentagonal ring system built from the lithium atoms of the amide. The charge balance is ensured by the solvens separated lithium cation, which is coordinated by two tmeda ligands, in the same unit cell. In contrast to conventional inverse crown ethers, which are mainly based on heterobimetallic complexes, in both related structures of this thesis the captured anion is coordinated by lithium atoms only.

The Simpkin's amide $(R, R)-\left\{[\mathrm{Ph}(\mathrm{Me}) \mathrm{CH}]_{2} \mathrm{NLi}\right\}$.can be regarded as a chiral analog of parent LDA. So far all attempts to rationalize the reaction mechanism of the asymmetric deprotonations by this lithium amide failed, but agostic interactions between the methyl groups of the amidic side chains and the lithium atom have been accredited to be a structure 
determining factor, which should influence the transition state, too. While these secondary interactions could not be retrieved by Andrews et al. in the crystal structure of monomeric $(R, R)-\left\{[\mathrm{Ph}(\mathrm{Me}) \mathrm{CH}]_{2} \mathrm{NLi} \cdot \mathrm{pmdeta}\right\},{ }^{[140]}$ this could be founded in the strong tricoordination of lithium by the bulky pmdeta ligand. In the present work, the molecular structure of $(R, R)-$ $\left\{[\mathrm{Ph}(\mathrm{Me}) \mathrm{CH}]_{2} \mathrm{NLi} \cdot \mathrm{tmeda}\right\}$ (4) was revealed. Surprisingly, the didentate tmeda ligand is able to generate the monomer also. Furthermore, no $\beta-\mathrm{H}$ elimination as in the related LDA did occur. Although the steric demand of tmeda and the ability of two nitrogen donors to saturate the charge depletion on the lithium atom are substantially reduced in comparison to pmdeta, the carbon-lithium distances to the methyl groups are even longer than in the pmdeta analog. For this reason, agostic interactions seem not to influence the conformation of the chiral amidic side chains. Therefore, their importance in a postulated transition state model is questionable, too.

Recently, heterobimetallic ate complexes have become the focus of organometallic chemistry, since their reactivity and chemoselectivity outperforms their monometallic congeners in many cases. ${ }^{[43]}$ To describe fascinating new reaction patterns like the metadeprotonation of aromatic compounds, terms like "bimetallic cooperative effects" or "synergistic effects" have been coined. To recover the origin of these, trimethylzincate as the root compound of triorganozincates has been chosen. Due to the high reactivity, only crystal structures of trialkylzincates with bulky stabilizing substituents are known. However, these are inappropriate model compounds since their molecular structure is altered significantly compared to chemically interesting zincate complexes with small ligands. During this thesis three molecular structures of trimethylzincate were elucidated by cryo X-ray crystal structure determination. In the presence of the tridentate ligand diglyme, the solvens-separated ion pair (SSIP) [(diglyme) $\left.)_{2} \mathrm{Li}\right]\left[\mathrm{ZnMe}_{3}\right](5)$ is produced. Since the proximity of both metals is not ensured in the SSIP, synergistic effects cannot be exerted. On the contrary, replacing diglyme by the tridentate amine ligand pmdeta yields the contact ion pair (CIP) [(pmdeta)Li( $\mu$ $\mathrm{Me})_{2} \mathrm{ZnMe}$ (6), the zinc and lithium atoms being $\mu$-bridged by one methyl group. This affords the necessary precondition for bimetallic cooperative effects to arise. Since the solid state structures obtained from X-ray data do not necessarily coincidence with solution structures, ${ }^{1} \mathrm{H}$ Diffusion ordered NMR experiments (DOSY) were performed. These clearly show that the SSIP as well as the CIP are retained in solution. However, the control of CP vs. SSIP is very sensitive towards further lithium containing salts present in solution. The determined crystal structure of $\left[(\text { pmdeta })_{2}\left(\mathrm{Li}_{2} \mathrm{Cl}\right)\right]\left[\mathrm{ZnMe}_{3}\right](7)$ reveals, that traces of lithium chloride can destroy the CIP and result in a SSIP. Adjoining theoretical calculations on the relative stabilities of modeled CIPS and SSIPS of trimethylzincate in the presence of diglyme and pmdeta exhibited that the formation of the respective aggregates can be predicted correctly. 
In the next step the computational studies were extended to the analysis of bond properties in order to find evidence for the postulated synergistic effects. For this purpose, QTAIM and NBO studies on neutral dimethylzinc, the trimethylzincate anion as a model for a SSIP and the CIP 6 have been performed, to monitor the change in bond characteristics. In fact, the zinc-carbon in $\mathrm{Me}_{3} \mathrm{Zn}^{-}$is weaker than in the divalent organozincate. In the CIP, a further decrease in bond strength is observed for only one of three zinc-carbon bonds, which is involved in an agostic interaction to the lithium atom. The two remaining bonds are not affected in the CIP: It can be concluded, that the contact of one methyl group of the zincate to lithium is vital for its activation in deprotonation reactions.

The second topic of this thesis is of purely theoretical and academical nature. It concerns the concept of hypervalency, which describes the fact that many third and higher row elements acquire coordination numbers, which imply a violation of the Lewis octet rule. Ever since the bonding in hypervalent compounds has been regarded as exceptional and it has been described by d-orbital participation, ionic resonance forms preserving the Lewis octet or many center electron bonding. In the present work, QTAIM and ELI are applied to this old problem in a combined way. While QTAIM offers the definition of an atom basin in a molecule, ELI partitions the space in a molecule in regions, where the localization of electron pairs is very high. On the one hand the number of electrons in a QTAIM basin yields atomic partial charges. On the other hand by integrating the average electron number of disynaptic ELI basins, the bond order can be deduced. The hybridization and number of electron pairs of an atom becomes visible by examination of the electron population of the monosynaptic ELI basins. Afterwards the polarity of a bond between two atoms can be determined quantitatively by intersecting the disynaptic bonding ELI basin with the atomic QTAIM basins. This procedure reveals the portioning of the bonding electrons between the participating atoms. The four so-called hypervalent molecules $\mathrm{S}(\mathrm{NH})_{3} 10, \mathrm{H}_{3} \mathrm{PO}(11), \mathrm{SO}_{4}{ }^{2-}(12)$ and $\mathrm{PO}_{4}{ }^{3-}$ (13) were envisaged. In a notion that avoids formal charges these molecules contain on ore more double bonds, which leads to a valence shell expansion on the central atom. It was shown that the sulfur-nitrogen bond in $\mathbf{1 0}$ features a remarkable $\pi$-contribution and has a moderate polarity at all. On the contrary, the phosphor- and sulfur-oxygen bonds in 11-13 exhibit bond orders below one and show a very high polarity. Furthermore, no hints for $\pi-$ backbonding through negative hyperconjugation or for banana bonds were detected. 


\section{ZUSAMMENFASSUNG UND AUSBLICK}

Die vorliegende Arbeit behandelt zwei Themengebiete. Im ersten Teil geht es um die Synthese und Kristallisation reaktiver ogranometallischer Deprotonierungsreagentien sowie wichtiger metallierter Intermediate, die im Reaktionsverlauf entstehen können. Diese extrem luft- und feuchtigkeitsempfindlichen Kristalle wurden im nächsten Schritt mithilfe des X-Temp-2 Systems ${ }^{[11]}$ bei $-100^{\circ} \mathrm{C}$ auf ein Röntgendiffraktometer appliziert. Dabei wurde die erste Kristallstruktur eines monomeren lithiierten Anisols aufgeklärt. Dieses Molekül stellt eine wichtige Zwischenstufe bei der dirigierenden ortho Metallierung (DoM) dar. Es wird angenommen, dass das lithiierte Intermediat durch eine Wechselwirkung zwischen der Methoxygruppe des Anisols und dem benachbarten Lithium Atom stabilisiert wird. Um den stabilisierenden Effekt zu analysieren und zu quantifizieren, wurden ausgehend von den Kristallkoordinaten quantenchemische Rechnungen durchgeführt. Hierzu wurden die relativ neuen Bindungsanalysetools QTAIM und ELI eingesetzt. Beide treffen die gleiche Aussage, dass keine attraktiven Wechselwirkungen zwischen den freien Elektronenpaaren des Sauerstoffatoms und dem Lithiumkation auszumachen sind. Während Lithium-Sauerstoff Wechselwirkungen in einem CIPE (complex induced proximity effect) Praekomplex noch kontrovers diskutiert werden, scheint es so, dass sie zumindest im ortho-lithiierten Anisol keine grössere Rolle mehr spielen.

Die zweite untersuchte Verbindung stellt ein gemischtes Aggregat aus einer Reaktion von Methyllithium mit Lithium-tert-butanolat dar. Komplexe dieser Art zeigen teilweise eine dramatisch erhöhte Reaktivität gegenüber konventionellen Organolithiumreagentien, weswegen sie auch als Lochman-Schlosser Superbasen bezeichnet werden. Im vorliegenden Fall konnte gezeigt werden, dass diese Reagenzien auch die Fähigkeit besitzen, Sauerstoffatme einzufangen. Die bestimmte Kristallstruktur zeigt einen dreidimensionalen inversen Kronenether, der aus zehn tBuOLi und vier MeLi Einheiten besteht, die zwei $\mathrm{O}^{2-}$ Anionen einkapseln. Die Koordinationszahl des Sauerstoffdianions und die Art der Wechselwirkungen zu den solvatisierenden Lithiumatomen wurden durch theoretische QTAIM Untersuchungen bestimmt.

Eine weitere inverse Kronenetherstrukur wurde durch die Zugabe des Diaminliganden Tmeda zu Lithiumdiisopropylamid (LDA) erhalten. Vermutlich aus der $\beta-\mathrm{H}$ Abstraktion eines LDA Moleküls entstandenes Lithiumhydrid wird von überschüssigem LDA solvatisiert. Dabei wird das Hydridion innerhalb eines aus Lithium Atomen des Amids bestehenden pentagonalen Ringsystems eingefangen. Der Ladungsausgleich wird durch das solvensseparierte Lithiumkation in derselben Elementarzelle gewährleistet, das von zwei Tmeda Liganden koordiniert wird. Im Gegensatz zu herkömmlichen inversen Kronenethern, die zumeist aus heterobimetallischen Komplexen bestehen, wird das eingelagerte Anion in 
den beiden verwandten Strukturen dieser Arbeit ausschliesslich von Lithiumatomen koordiniert.

Das Simpkin's Amid $(R, R)-\left\{[\mathrm{Ph}(\mathrm{Me}) \mathrm{CH}]_{2} \mathrm{NLi}\right\}$ kann als chirales Analogon zur Stammverbindung LDA betrachtet werden. Bis zum gegenwärtigen Zeitpunkt scheiterten alle Versuche, den Reaktionsmechanismus für die asymmetrische Deprotonierung dieses Lithiumamids zu verstehen, jedoch wurden agostische Wechselwirkungen zwischen den Methylgruppen der Amidseitenketten und dem Lithiumatoms als strukturbestimmender Faktor bezeichnet, der auch den Übergangszustand beeinflussen sollte. Zwar konnten Andrews et al. diese sekundären Wechselwirkungen anhand der Kristallstrukutur des monomeren $(R, R)-\left\{[\mathrm{Ph}(\mathrm{Me}) \mathrm{CH}]_{2} \mathrm{NLi} \cdot\right.$ pmdeta $\}$ nicht nachvollziehen, ${ }^{[140]}$ was allerdings an der starken Dreifachkoordination des Lithium Atoms durch den sperrigen Pmdeta Liganden liegen könnte.

In der vorliegenden Arbeit wurde die Molekülstruktur von $(R, R)-\left\{[\mathrm{Ph}(\mathrm{Me}) \mathrm{CH}]_{2} \mathrm{NLi} \cdot \operatorname{tmeda}\right\}$ (4) aufgeklärt. Überraschenderweise ist der zweizähnige Ligand Tmeda ebenfalls in der Lage, ein Monomer zu generieren. Desweiteren ist zu erwähnen, dass keine $\beta-\mathrm{H}$ Eliminierung wie im verwandten LDA auftrat. Obwohl der sterische Anspruch Tmedas und die Fähigkeit seiner lediglich zwei Stickstoffdonoren, die Ladungsverarmung am Lithiumatom auszugleichen, im Vergleich zu Pmdeta beträchtlich verringert ist, sind die KohlenstoffLithium Abstände zu den Methylgruppen sogar länger als im Pmdeta Analogon. Aus diesen Gründen scheinen agostische Wechselwirkungen die Konformation der chiralen Seitenketten nicht $\mathrm{zu}$ beeinflussen. Daher ist ihre Bedeutung in einem postulierten Übergangszustandsmodell ebenfalls fragwürdig.

In letzter Zeit sind heterobimetallische At-Komplexe immer stärker in den Fokus der Organometallchemie gerückt, da sie ihre monometallischen Vorgänger in punkto Reaktivität und Chemoselektivität oftmals um ein Vielfaches übertreffen. ${ }^{[43]}$ Begriffe wie "bimetallisch kooperative Effekte" oder "synergistische Effekte" wurden angeführt, um faszinierende neue Reaktionsmuster wie die meta-Deprotonierung aromatischer Verbindungen zu erklären. Um deren Ursprung aufzuklären, wurde Trimethylzinkat als Basisverbindung aller Triorganozinkate ausgewählt. Wegen ihrer hohen Reaktivität sind lediglich Kristallstrukturen von Trialkylzinkaten mit sperrigen stabilisierenden Substituenten bekannt. Diese eignen sich jedoch schlecht als Modellverbindungen, da ihre Molekularstruktur im Vergleich zu Zinkatkomplexen mit kleineren Liganden, die auch für die chemische Synthese interessant sind, in grossem Masse verschieden ist. Im Laufe dieser Arbeit wurden drei Molekülstrukturen von Trimethylzinkaten mithilfe von Kryo-Röntgenkristallstrukturbestimmung aufgeklärt. In Gegenwart des dreizähnigen Liganden Diglyme entsteht das solvensseparierte lonenpaar (SSIP) [(diglyme) $\left.{ }_{2} \mathrm{Li}\right]\left[\mathrm{ZnMe}_{3}\right](5)$. Da die räumliche Nähe der Metalle im SSIP nicht gewährleistet ist, können synergistische Effekte nicht ausgespielt 
werden. Im Gegensatz dazu führt ein Austauch von Diglyme gegen den dreizähnigen Aminliganden Pmdeta zum Kontaktionenpaar (CIP) [(pmdeta)Li( $\left.\mu-\mathrm{Me})_{2} \mathrm{ZnMe}\right](6)$, in dem Zink- und Lithiumatom durch eine Methylgruppe überbrückt werden. Dies stellt die notwendige Voraussetzung dar, damit bimetallisch koopertive Effekte auftreten können. Da Festkörperstrukturen, die aus Röntgenstrukturdaten erhalten wurden, nicht notwendigerweise mit den Strukturen in Lösung übereinstimmen, wurden ${ }^{1} \mathrm{H}$ DiffusionOrdered NMR Experimente (DOSY) durchgeführt. Diese ergeben eindeutig, dass sowohl das SSIP als auch das CIP in Lösung erhalten bleiben. Die Steuerung CIP vs. SSIP ist allerdings sehr empfindlich gegenüber weiteren Lithiumsalzen, die in Lösung vorhanden sind. Die Aufklärung der Kristallstruktur von [(pmdeta $\left.)_{2}\left(\mathrm{Li}_{2} \mathrm{Cl}\right)\right]\left[\mathrm{ZnMe}_{3}\right](7)$ legt nahe, dass Spuren von Lithiumchlorid das CIP zerstören können und zur Bildung des SSIP führen. Begleitende theoretische Rechnungen zur Bestimmung der relativen Energien modellierter CIPs und SSIPs des Trimethylzinkates in Gegenwart von Diglyme und Pmdeta zeigten, dass die Bildung der jeweiligen Aggregate korrekt vorhergesagt werden kann.

Im nächsten Schritt wurden die computerchemischen Untersuchungen auf die Analyse von Bindungseigenschaften ausgeweitet, um Belege für die postulierten synergistischen Effekte zu finden. Hierzu wurden QTAIM und NBO Studien an neutralem Dimethylzink, dem Trimethylzinkatanion als einem Modell für das SSIP, und dem CIP 6 durchgeführt, um Veränderungen in den Bindungsgegebenheiten zu verfolgen. Tatsächlich ist die ZinkKohlenstoff Bindung in $\mathrm{Me}_{3} \mathrm{Zn}^{-}$gegenüber dem divalenten Organozinkat geschwächt. Im CIP kann für eine der drei Zink-Kohlenstoff Bindungen eine Schwächung beobachtet werden, welche eine agostische Wechselwirkung zu dem Lithiumatom ausbildet. Die zwei übrigen Bindungen im CIP sind davon nicht betroffen. Es kann gemutmasst werden, dass der Kontakt einer Methylgruppe des Zinkates zu Lithium für seine Aktivierung in Deprotonierungsreaktionen entscheidend ist.

Das zweite Thema dieser Dissertation ist rein theoretischer und akademischer Natur. Es betrifft das Konzept der Hypervalenz, welches die Tatsache beschreibt, dass zahlreiche Elemente der höheren Perioden Koordinationszahlen erreichen, die eine Verletzung der Lewis Oktett Regel nahelegen. Seit jeher wurde die Bindungssituation in hypervalenten Verbindungen als ungewöhnlich betrachtet und mit d-Orbitalbeteiligung, ionischen Resonanzstrukturen, die das Lewis Oktett einhalten, und Mehrfachzentrenbindungen erklärt.

In der vorliegenden Arbeit wurden QTAIM und ELI in kombinierter Weise auf dieses alte Problem angesetzt. Während QTAIM die Definition für Atombasins in Molekülen liefert, teilt die ELI den Raum eines Moleküles in Regionen hoher Lokalisierung von Elektronenpaaren auf. Zum einen liefert die Anzahl der Elektronen innerhalb eines QTAIM Basins atomare Partialladungen. Zum anderen kann durch Integration der durchschnittlichen Elektronenanzahl eines disynaptischen ELI Basins die Bindungsordnung abgeleitet werden. 
Die Hybridisierung und Anzahl freier Elektronenpaare eines Atoms wird nach Auswertung der Elektronenpopulation der monosynaptischen ELI Basins erkennbar. Anschliessend kann die Polarität der Bindung quantitativ aus der Schnittmenge der disynaptischen ELI Bindungsbasins mit den atomaren QTAIM Basins bestimmt werden. Diese Vorgehensweise offenbart die Aufteilung der bindenden Elektronen auf die beteiligten Atome.

Die vier sogenannten hypervalenten Moleküle $\mathrm{S}(\mathrm{NH})_{3} 10, \mathrm{H}_{3} \mathrm{PO}(11), \mathrm{SO}_{4}{ }^{2-}$ (12) und $\mathrm{PO}_{4}{ }^{3-}$ (13) wurden ausgewählt. In einer Schreibweise, die Formalladungen vermeidet, enthalten diese Moleküle eine oder mehrere Doppelbindungen, was zu einer Valenzaufweitungen am Zentralatom führt. Es wurde gezeigt, dass die SchwefelStickstoffbindung in 10 einen beträchtlichen m-Beitrag enthält und über eine moderate Polarität verfügt. Im Gegensatz dazu sind die Bindungsordnungen für 11-13 unterhalb von eins und weisen eine sehr hohe Polarität auf. Desweiteren wurden keine Hinweise für $\pi-$ Rückbindungen durch negative Hyperkonjugation oder Banana Bonds gefunden. 


\section{EXPERIMENTAL SECTION}

\subsection{General Procedure}

All experiments were carried under inert condition (purified and dried argon atmosphere) using modified Schlenk techniques. Solid air and moisture sensitive compounds were handled in a MBraun LABmaster sp argon glove box. All glass devices were dried at $120^{\circ} \mathrm{C}$ for several hours, before they were used for chemical reactions. The solvents were freshly distilled from sodium-potassium alloy ( $\mathrm{Et}_{2} \mathrm{O}, n$-pentane) or potassium (dem, thf, $n$-hexane) and degassed. The starting materials were purchased by Sigma-Aldrich or Acros Organics, donated by the Chemetall $A G$ or prepared according to literature procedures. All organometallic compounds of this thesis were extremely reactive and thermally instable. Therefore they were stored under protective atmosphere at low temperatures $\left(-18\right.$ till $\left.-45^{\circ} \mathrm{C}\right)$.

\subsection{Analytical Methods}

\subsubsection{NMR spectroscopy}

The crystalline solids were transferred into an argon glove box, where they were filled up into special NMR tubes with and attached Schlenk valve. Over sodium or potassium dried deuterated solvents (benzene, toluene) were added outside the glove box under inert conditions. Subsequently, the special NMR tube was frozen in liquid nitrogen, evacuated and melted away from the attached Schlenk valve with a Bunsen burner. Immediately afterwards the spectra were recorded at room temperature on Bruker Avance 300 and 500 spectrometers. The measurement frequencies and solvents are given in the particular analysis. The chemical shifts $\delta$ are given in ppm with negative values for high-field shifts against tetramethylsilane $\left({ }^{1} \mathrm{H},{ }^{13} \mathrm{C}\right)$ or lithium chloride in $\mathrm{D}_{2} \mathrm{O}\left({ }^{7} \mathrm{Li}\right)$.

\subsubsection{Elemental Analyses}

Elemental analyses were kindly measured at an Elementar Vario EL3 by the Mikroanalytische Labor des Instituts für Anorganische Chemie der Georg-August-Universität Göttingen. Therefore the samples were prepared in an argon glove box. Still, due to decomposition variances to calculated values can occur. In addition, inclusion of argon in the sample probes leads to small systematic errors. 


\subsection{Syntheses and Characterizations}

\subsubsection{2-AnisoLi-pmdeta (1)}

A solution of $n$-butyllithium in hexane $(22 \mathrm{~mL}, 55 \mathrm{mmol})$ was added slowly to a solution of anisole $(5.42 \mathrm{~mL}, 50 \mathrm{mmol})$ in hexane $(10 \mathrm{~mL})$. Subsequently, pmdeta $(10.44 \mathrm{~mL}, 50 \mathrm{mmol})$ was added and the reaction mixture was heated to $40-50{ }^{\circ} \mathrm{C}$ for $2 \mathrm{~h}$. Crystallization was carried out in a freezer at $-24{ }^{\circ} \mathrm{C}$ and light brown crystals $(4.7 \mathrm{~g}, 87 \%)$ were obtained.

Empirical Formula: $\mathrm{C}_{16} \mathrm{H}_{30} \mathrm{LiN}_{3} \mathrm{O} \quad$ Molecular weight: $287.37 \mathrm{~g} \mathrm{~mol}^{-1}$

${ }^{1} \mathrm{H}$ NMR $\left(\mathbf{5 0 0 . 1 3} \mathbf{~ M H z}, \mathrm{C}_{6} \mathrm{D}_{6}, \mathbf{2 5}^{\circ} \mathrm{C}\right): \delta=2.03\left(\mathrm{~s}, 12 \mathrm{H}, \mathrm{N}\left(\mathrm{CH}_{3}\right)_{2}\right), 2.09\left(\mathrm{~s}, 3 \mathrm{H}, \mathrm{NCH}_{3}\right), 2.17$ (bs, $8 \mathrm{H}, \mathrm{NCH} 8.21(1 \mathrm{H}$, bs) ppm.

${ }^{7} \mathrm{Li}\left\{{ }^{1} \mathrm{H}\right\}-N M R\left(116.64 \mathrm{MHz}, \mathrm{C}_{6} \mathrm{D}_{6}, 25^{\circ} \mathrm{C}\right): \delta=2.04 \mathrm{ppm}$.

${ }^{13} \mathrm{C}\left\{{ }^{1} \mathrm{H}\right\}-N M R\left(125.76 \mathrm{MHz}, \mathrm{C}_{6} \mathrm{D}_{6}, 25^{\circ} \mathrm{C}\right): \delta=43.8\left(\mathrm{~N}\left(\mathrm{CH}_{3}\right), 45.8\left(\mathrm{~N}\left(\mathrm{CH}_{3}\right)_{2}\right), 54.0\left(\mathrm{NCH}_{2}\right)\right.$, $55.3\left(\mathrm{OCH}_{3}\right), 57.6\left(\mathrm{NCH}_{2}\right), 106.4(\mathrm{PhC}), 114.2(\mathrm{PhC}), 120.8(\mathrm{PhC}), 129.6(\mathrm{PhC}), 142.8$ (PhC), $169.8(\mathrm{PhC}) \mathrm{ppm}$.

Elemental analysis calculated for $\mathrm{C}_{16} \mathrm{H}_{30} \mathrm{LiN}_{3} \mathrm{O}$ : C 66.87; $\mathrm{H} 10.52, \mathrm{~N}, 14.62$; found $\mathrm{C}$ 64.36, H 11.24, N $15.77 \%$.

\subsubsection{Scavenger (2)}

A solution of methyllithium in diethyl ether $(20.2 \mathrm{~mL}, 27.0 \mathrm{mmol})$ was added slowly to a solution of tert-BuOH $(1.20 \mathrm{~mL}, 13.5 \mathrm{mmol})$ in diethyl ether $(10.0 \mathrm{~mL})$ at $0{ }^{\circ} \mathrm{C}$. A white solid precipitated and was filtered off. The filtrate was stored at $0{ }^{\circ} \mathrm{C}$ and after one week, a small quantity of colorless crystals deposited from the solution.

Empirical Formula: $\mathrm{C}_{44} \mathrm{H}_{102} \mathrm{Li}_{18} \mathrm{O}_{12} \quad$ Molecular weight: $948.21 \mathrm{~g} \mathrm{~mol}^{-1}$ ${ }^{1} \mathrm{H}$ NMR $\left(\mathbf{5 0 0 . 1 3} \mathrm{MHz}, \mathrm{C}_{6} \mathrm{D}_{6}, 25^{\circ} \mathrm{C}\right): \delta=-1.28(\mathrm{sb}, 12 \mathrm{H}), 1.27(\mathrm{~s}, 90 \mathrm{H}) \mathrm{ppm}$.

${ }^{7}$ Li\{ $\left.{ }^{1} \mathrm{H}\right\}-N M R\left(116.64 \mathrm{MHz}, \mathrm{C}_{6} \mathrm{D}_{6}, \mathbf{2 5}^{\circ} \mathrm{C}\right): \delta=0.69,0.91,1.14,1.31,1.75,1.85,1.93 \mathrm{ppm}$.

${ }^{13} \mathrm{C}\left\{{ }^{1} \mathrm{H}\right\}$-NMR $\left(125.76 \mathrm{MHz}, \mathrm{C}_{6} \mathrm{D}_{6}, \mathbf{2 5}^{\circ} \mathrm{C}\right): \delta=35.4\left(\left(\mathrm{CH}_{3}\right)_{3} \mathrm{C}\right), 65.9\left(\left(\mathrm{CH}_{3}\right)_{3} \mathrm{C}\right) \mathrm{ppm}$.

The bridging methanide carbon atoms could not be detected in the ${ }^{13} \mathrm{C}-\mathrm{NMR}$.

\subsubsection{Hydrid (3)}

A solution of $n$-butyllithium in hexane $(14.6 \mathrm{~mL}, 32.85 \mathrm{mmol})$ was added slowly to a solution of diisopropylamine $(4.20 \mathrm{~mL}, 29.88 \mathrm{mmol})$ in hexane $(7.50 \mathrm{~mL})$ at $0{ }^{\circ} \mathrm{C}$, resulting in viscous syrup. After storing the reaction mixture over night at $2^{\circ} \mathrm{C}$, a white precipitate was 
formed. The solvent was removed by a syringe and the solid washed with cold hexane. Subsequently, the solid was redissolved in excess tmeda and filtered off. The filtrate was stored at $2{ }^{\circ} \mathrm{C}$. After a further week the formation of a red second phase was observed. One week later red crystals were obtained. Unfortunately, this obtained crystal modification could only be obtained once. Attempts to reproduce it always led to the literature known helical modification of LDA. ${ }^{[128]}$

Empirical Formula: $\mathrm{C}_{42} \mathrm{H}_{103} \mathrm{Li}_{6} \mathrm{~N}_{9}$

Molecular weight: $559.96 \mathrm{~g} \mathrm{~mol}^{-1}$

\subsubsection{Simpkin's amide (4)}

A solution of $n$-butyllithium in hexane $(4.5 \mathrm{~mL}, 9.69 \mathrm{mmol})$ was added slowly to a solution of $(R, \mathrm{R})-(+)$-bis(1-methylbenzyl)amine $(2.00 \mathrm{~mL}, 8.74 \mathrm{mmol})$ in hexane $(10 \mathrm{~mL})$. The reaction mixture was stored at $-18{ }^{\circ} \mathrm{C}$ for four days. Subsequently, the solvent was removed with a syringe, the precipitate was washed with cold hexane a redissolved in tmeda. After one week, dark red crystals were obtained $(2.3 \mathrm{~g}, 74 \%)$.

Empirical Formula: $\mathrm{C}_{22} \mathrm{H}_{34} \mathrm{LiN}_{3}$

Molecular weight: $347.47 \mathrm{~g} \mathrm{~mol}^{-1}$

${ }^{1} \mathrm{H}$ NMR (500.13 MHz, $\left.\mathbf{C}_{6} \mathrm{D}_{6}, \mathbf{2 5}^{\circ} \mathrm{C}\right): \delta=1.14\left(\mathrm{~d}, 6 \mathrm{H}, \mathrm{C}(\mathrm{H}) \mathrm{CH}_{3},{ }^{3} \mathrm{~J}=6.7 \mathrm{~Hz}\right), 2.09(\mathrm{~s}, 12 \mathrm{H}$, $\mathrm{NCH}_{3}$ ), $2.28\left(\mathrm{~s}, 4 \mathrm{H}, \mathrm{NCH}_{2}\right.$ ), $3.46\left(\mathrm{q}, 2 \mathrm{H}, \mathrm{CH},{ }^{3} \mathrm{~J}=6.6 \mathrm{~Hz}\right), 7.05-7.20(\mathrm{~m}, 10 \mathrm{H}, \mathrm{PhH}) \mathrm{ppm}$.

${ }^{7} \mathrm{Li}\left\{{ }^{1} \mathrm{H}\right\}-N M R\left(116.64 \mathrm{MHz}, \mathrm{C}_{6} \mathrm{D}_{6}, 25^{\circ} \mathrm{C}\right): \delta=2.01 \mathrm{ppm}$.

${ }^{13} \mathrm{C}\left\{{ }^{1} \mathrm{H}\right\}-\mathrm{NMR}\left(125.76 \mathrm{MHz}, \mathbf{C}_{6} \mathrm{D}_{6}, 2{ }^{\circ} \mathrm{C}\right): \delta=32.0\left(\mathrm{CH}_{3}\right), 45.9\left(\mathrm{NCH}_{3}\right), 55.5\left(\mathrm{NCH}_{2}\right), 58.3$ $(\mathrm{CH}), 126.8(\mathrm{CH}), 126.9(\mathrm{CH}), 128.6(\mathrm{CH}), 146.6(\mathrm{CH}) \mathrm{ppm}$.

\subsection{5 $\mathrm{Me}_{3} \mathrm{ZnLi} \cdot d i g l y m e ~(5)$}

A solution of methyllithium in diethyl ether $(7.5 \mathrm{~mL}, 12.0 \mathrm{mmol})$ was slowly added to a solution of dimethylzinc in toluene $(5 \mathrm{~mL}, 10.0 \mathrm{~mL})$ at $0^{\circ} \mathrm{C}$. Thereby, a white solid precipitated. The reaction mixture was stirred for $2.5 \mathrm{~h}$ at $0{ }^{\circ} \mathrm{C}$, and then diglyme $(2.1 \mathrm{~mL}$, $14.8 \mathrm{mmol}$ ) was added. The precipitate was filtered off and the crystallization was carried out in the freezer at $-45^{\circ} \mathrm{C}$. Yields of crystalline product: $1.4 \mathrm{~g} \mathrm{(36 \% )}$.

Empirical Formula: $\mathrm{C}_{15} \mathrm{H}_{37} \mathrm{LiO}_{6} \mathrm{Zn} \quad$ Molecular weight: $385.80 \mathrm{~g} \mathrm{~mol}^{-1}$

${ }^{1} \mathrm{H}-\mathrm{NMR}\left(\mathbf{5 0 0 . 1 3} \mathrm{MHz}, \mathrm{C}_{6} \mathrm{D}_{5} \mathrm{CD}_{3}, \mathbf{2 5}^{\circ} \mathrm{C}\right): \delta=-0.60\left(\mathrm{~s}, 9 \mathrm{H},\left(\mathrm{CH}_{3}\right)_{3} \mathrm{Zn}\right), 3.11\left(\mathrm{~s}, 12 \mathrm{H},\left(\mathrm{OCH}_{3}\right)\right)$, $3.28\left(\mathrm{~m}, 8 \mathrm{H}, \mathrm{CH}_{2}\right), 3.39\left(\mathrm{~m}, 8 \mathrm{H}, \mathrm{CH}_{2}\right) \mathrm{ppm}$.

$\left.{ }^{7} \mathrm{Li}^{2}{ }^{1} \mathrm{H}\right\}-N M R\left(194.37 \mathrm{MHz}, \mathrm{C}_{6} \mathrm{D}_{5} \mathrm{CD}_{3}, 25^{\circ} \mathrm{C}\right): \delta=0.05(\mathrm{~s}) \mathrm{ppm}$. 
${ }^{13} \mathrm{C}\left\{{ }^{1} \mathrm{H}\right\}-N M R\left(125.76 \mathrm{MHz}, \mathrm{C}_{6} \mathrm{D}_{5} \mathrm{CD}_{3}, 25^{\circ} \mathrm{C}\right): \delta=-7.23\left(\left(\mathrm{CH}_{3}\right)_{3} \mathrm{Zn}\right), 58.60\left(\mathrm{OCH}_{3}\right), 70.79$ $\left(\mathrm{CH}_{2}\right), 72.21\left(\mathrm{CH}_{2}\right) \mathrm{ppm}$.

Elemental analysis calculated for $\mathrm{C}_{15} \mathrm{H}_{37} \mathrm{LiO}_{6} \mathrm{Zn}$ : $\mathrm{C}, 46.70 ; \mathrm{H}, 9.67$; found $\mathrm{C} 46.34 ; \mathrm{H}$ $9.83 \%$.

\subsection{6 $\mathrm{Me}_{3} \mathrm{ZnLi} \cdot \mathrm{pmdeta}(6)$}

A solution of methyllithium in diethyl ether $(7.5 \mathrm{~mL}, 12.0 \mathrm{mmol})$ was slowly added to a solution of dimethylzinc in toluene $(5 \mathrm{~mL}, 10.0 \mathrm{mmol})$ at $0{ }^{\circ} \mathrm{C}$. Thereby, a white solid precipitated. The reaction mixture was stirred for $2.5 \mathrm{~h}$ at $0{ }^{\circ} \mathrm{C}$, and then pmdeta $(2.4 \mathrm{~mL}$, $14.8 \mathrm{mmol}$ ) was added. The precipitate was filtered off and the crystallization was carried out in the freezer at $-45^{\circ} \mathrm{C}$. Yields of crystalline product: $1.3 \mathrm{~g}(45 \%)$.

Empirical Formula: $\mathrm{C}_{16} \mathrm{H}_{30} \mathrm{LiN}_{3} \mathrm{O} \quad$ Molecular weight: $287.37 \mathrm{~g} \mathrm{~mol}^{-1}$

${ }^{1} \mathrm{H}-\mathrm{NMR}\left(500.13 \mathrm{MHz}, \mathrm{C}_{6} \mathrm{D}_{5} \mathrm{CD}_{3}, 25^{\circ} \mathrm{C}\right): \delta=-0.48\left(\mathrm{~s}, 9 \mathrm{H},\left(\mathrm{CH}_{3}\right)_{3} \mathrm{Zn}\right), 1.68\left(\mathrm{~s}, \mathrm{br}, 8 \mathrm{H}, \mathrm{CH}_{2}\right)$, $1.84\left(\mathrm{~s}, \mathrm{br}, 3 \mathrm{H}, \mathrm{NCH}_{3}\right), 1.87\left(\mathrm{~s}, \mathrm{br}, 12 \mathrm{H}, \mathrm{N}\left(\mathrm{CH}_{3}\right)_{2}\right) \mathrm{ppm}$.

${ }^{7} \mathrm{Li}\left\{{ }^{1} \mathrm{H}\right\}-N M R\left(194.37 \mathrm{MHz}, \mathrm{C}_{6} \mathrm{D}_{5} \mathrm{CD}_{3}, 25^{\circ}\right): \delta=0.22$ (s) ppm.

${ }^{13} \mathrm{C}\left\{{ }^{1} \mathrm{H}\right\}$-NMR $\left(126.76 \mathrm{MHz}, \mathrm{C}_{6} \mathrm{D}_{5} \mathrm{CD}_{3}, 25^{\circ} \mathrm{C}\right): \delta=-6.46\left(\left(\mathrm{CH}_{3}\right)_{3} \mathrm{Zn}\right), 44.52\left(\mathrm{NCH}_{3}\right), 45.60$ $\left(\mathrm{N}\left(\mathrm{CH}_{3}\right)_{2}\right), 54.16\left(\mathrm{CH}_{2}\right), 57.16\left(\mathrm{CH}_{2}\right) \mathrm{ppm}$.

Elemental analysis calculated for $\mathrm{C}_{16} \mathrm{H}_{30} \mathrm{LiN}_{3} \mathrm{O}: \mathrm{C} 66.87, \mathrm{H} 10.52, \mathrm{~N} 14.62$; found $\mathrm{C} 65.43, \mathrm{H}$ $11.44, \mathrm{~N} 16.07 \%$. 


\section{Crystallographic Section}

\subsection{Crystal Application}

For the data acquisition the air and moisture sensitive crystals were selected at low temperatures using Schlenk techniques and the X-Temp 2 system. ${ }^{[11]}$ Thereby, the flask containing the crystals, which were freed from their mother liquor, was constantly kept at low temperatures and connected to a Schlenk line available at a special mounting table in the diffractometer room. The crystals were transferred into a perfluorinated oil on the microscope slide, which was cooled to $-100{ }^{\circ} \mathrm{C}$ by a cold nitrogen stream of the X-Temp 2 . There, crystals of appropriate size were selected and their quality was checked by a polarization filter to detect twinned or split crystals. Then, the crystals were fixed in a drop of oil at the tip of a fibre and applied to the X-ray diffractometer, where they were shock-cooled by the crystal cooling device.

\subsection{Data collection and processing}

Data were collected on Bruker diffractometers with D8 goniometer and APEXII detector at $100 \mathrm{~K}$ (Mo Ka radiation, $\lambda=71.073 \mathrm{pm}$ with micro focus sealed tube equipped with INCOATEC Quazar mirror optics or a TXS rotating Anode with INCOATEC Helios mirror optics). The data collection was monitored with the APEX2 program package. ${ }^{[196]}$ The cell determination was carried out either with the tools provided by the APEX2 software or in difficult cases (twinning, poor crystal quality) with Sheldrick's special cell search program CELL_NOW..$^{[197]}$ The data were integrated with SAINT ${ }^{[198]}$ and an empirical absorption correction with SADABS ${ }^{[199]}$ (for single crystals) or TWINABS ${ }^{[200]}$ (for twinned crystals) was applied. Determination of the space group and set up for the files for the structure solution was performed with XPREP. ${ }^{[201]}$

\subsection{Structure Solution and refinement}

The structures were solved by direct methods with SHELXS ${ }^{[202]}$ and refined by full-matrix least squares methods against $F^{2}$. All non-hydrogen atoms were refined with anisotropic displacement parameters. The hydrogen atoms were refined isotropically on calculated positions using a riding model with their $U_{\text {iso }}$ values constrained to 1.5 times the $U_{e q}$ of their pivot atoms for terminal $\mathrm{sp}^{3}$-carbon atoms and 1.2 times for all other carbon atoms.

Disordered moieties were refined using bond length restraints, rigid bond restraints, similarity restraints and ADP-restraints. The quality of the refinement was checked by the 
differences between the calculated and observed structure factors $F_{o b s}$ and $F_{c a l c .}$. The most significant criteria are the $R 1$ and the $w R 2$.

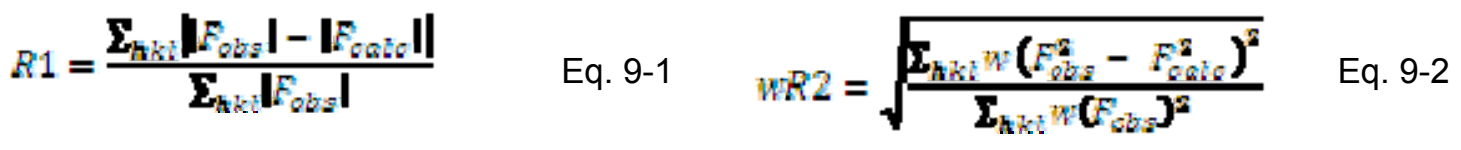

In the $w R 2$ the reflections are weighted by a factor $w$ with respect to the precision of their determination, as expressed by the estimated standard deviations (esd).

Furthermore, the GooF (goodness of fit) was analyzed, where the difference of the number of reflections $n$ and of the refined parameters $p$ is considered, too. While its value should be ideally one or slightly above, values lower than one indicate an over-determination of refined parameters, in other words the refined model shows a higher accuracy than the data quality provides.

$$
G 005=\sqrt{\frac{\sum_{h k i} n\left(F_{Q b 2}^{2}-F_{Q k i \alpha}^{2}\right)^{2}}{n-p}}
$$




\subsection{Crystallographic Details for 1-7}

\subsubsection{2-AnisoLi·pmdeta (1)}

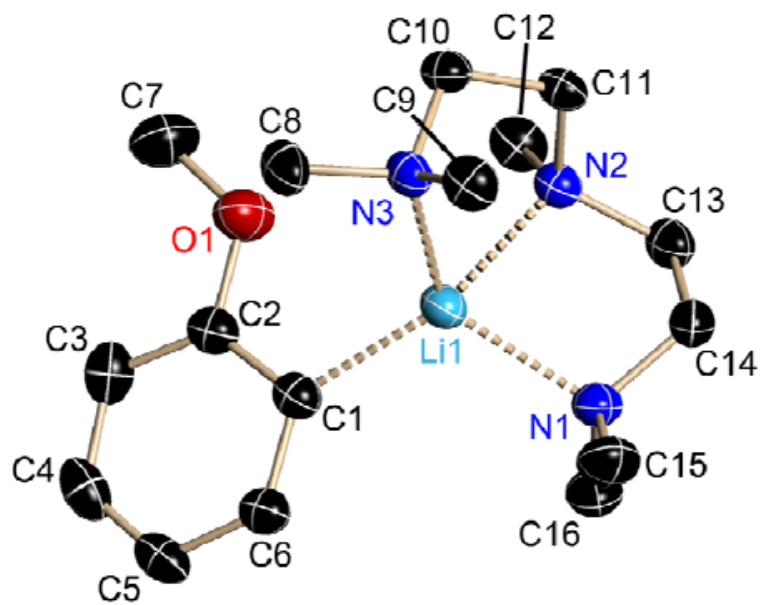

Figure 9-1. Asymmetric unit of 2-AnisoLi-pmdeta (1). Hydrogen atoms are omitted for clarity.

2-AnisoLi-pmdeta (1) crystallizes as light brown blocks in the monoclinic space group $P 2_{1} / n$ with the whole monomer in the asymmetric unit.

Table 9-1. Crystallographic data for 2-AnisoLi-pmdeta (1).

\begin{tabular}{ll|ll}
\hline identification code & AnisoLi-pmedta & & \\
empirical formula & $\mathrm{C}_{16} \mathrm{H}_{30} \mathrm{LiN}_{3} \mathrm{O}$ & $\mu\left[\mathrm{mm}^{-1}\right]$ & 0.065 \\
molar mass $\left[\mathrm{g} \mathrm{mol}^{-1}\right]$ & 287.37 & $\mathrm{~F}(000)$ & 632 \\
crystal size $[\mathrm{mm}]$ & $0.4 \times 0.2 \times 0.1$ & min./max. transmission & $0.9935 / 0.9744$ \\
temperature $[\mathrm{K}]$ & $100(2)$ & $\theta$ range $\left[^{\circ}\right]$ & $1.98-25.36$ \\
crystal system & monoclinic & completeness to $\theta_{\text {max }}$ & $99.8 \%$ \\
space group & $P 2_{1} / n$ & reflections collected & 27406 \\
$a[\AA]$ & $8.5759(9)$ & independent reflections & 3318 \\
$b[\AA]$ & $15.0426(16)$ & $\mathrm{R}_{\text {int }}$ & 0.0451 \\
$c[\AA]$ & $14.3945(15)$ & restraints / parameters & $0 / 196$ \\
$\alpha\left[^{\circ}\right]$ & 90 & GooF & 1.084 \\
$\beta\left[^{\circ}\right]$ & $102.1890(10)$ & $R 1($ all data) & 0.0617 \\
$\gamma\left[^{\circ}\right]$ & 90 & $R 1(I>2 \sigma(I))$ & 0.0516 \\
$V\left[\AA^{3}\right]$ & $1815.1(3)$ & $w R 2($ all data) & 0.1510 \\
$Z$ & 4 & $w R 2(\mathrm{I}>2 \sigma(\mathrm{I}))$ & 0.1420 \\
$\rho_{\text {calc }}\left[\mathrm{g} \mathrm{cm}{ }^{-3}\right]$ & 1.052 & diff. peak/hole $\left[\mathrm{e} \AA^{-3}\right]$ & $0.502 /-0.316$ \\
\hline
\end{tabular}




\subsubsection{Scavenger (2)}

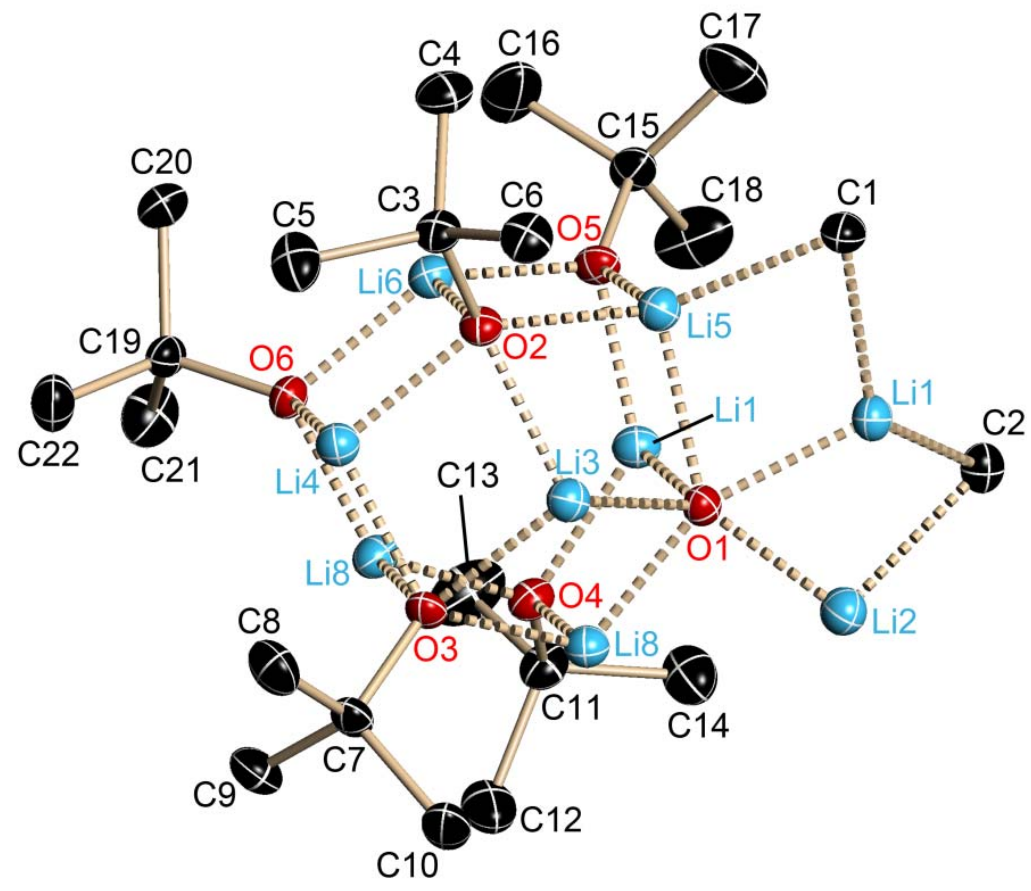

Figure 9-2. Asymmetric unit of Scavenger (2). Hydrogen atoms are omitted for clarity.

Scavenger (2) crystallizes as colorless blocks in the orthorhombic space group Pbca. The asymmetric unit contains only half of the molecule. The second half is generated by an inversion center. The hydrogen atoms of the methanide ions $\mathrm{C} 1$ and $\mathrm{C} 2$ were located in a difference Fourier map and refined without any constraints. 
Table 9-2. Crystallographic data for Scavenger (2).

\begin{tabular}{ll|ll}
\hline identification code & Scavenger & & \\
empirical formula & $\mathrm{C}_{22} \mathrm{H}_{51} \mathrm{Li}_{9} \mathrm{O}_{6}$ & $\mu\left[\mathrm{mm}^{-1}\right]$ & 0.063 \\
molar mass $\left[\mathrm{g} \mathrm{mol}^{-1}\right]$ & 474.09 & $F(000)$ & 2064 \\
crystal size $[\mathrm{mm}]$ & $0.38 \times 0.30 \times 0.19$ & min./max. transmission & $0.99 / 0.74$ \\
temperature $[\mathrm{K}]$ & $100(2)$ & $\theta$ range $\left[^{\circ}\right]$ & 1.91 to 25.38 \\
crystal system & Orthorhombic & completeness to $\theta_{\text {max }}$ & $99.5 \%$ \\
space group & $P b c a$ & reflections collected & 44935 \\
$a[\AA]$ & $1751.1(2)$ & independent reflections & 5850 \\
$b[\AA]$ & $1836.0(2)$ & $R_{\text {int }}$ & 0.0285 \\
$c[\AA]$ & $1990.9(2)$ & restraints / parameters & $36 / 398$ \\
$\alpha\left[^{\circ}\right]$ & 90 & GooF & 1.019 \\
$\beta\left[^{\circ}\right]$ & 90 & $R 1$ (all data) & 0.0428 \\
$\gamma\left[^{\circ}\right]$ & 90 & $R 1(>2 \sigma(I))$ & 0.0350 \\
$V\left[\AA^{3}\right]$ & $6400.5(13)$ & $w R 2($ all data) & 0.0933 \\
$Z$ & 8 & $w R 2(/>2 \sigma(I))$ & 0.0879 \\
$\rho_{\text {calc }}\left[\mathrm{g} \mathrm{cm}{ }^{-3}\right]$ & 0.984 & diff. peak/hole $\left[\mathrm{e} \AA^{-3}\right]$ & $0.197 /-0.153$ \\
\hline
\end{tabular}




\subsubsection{Hydrid (3)}

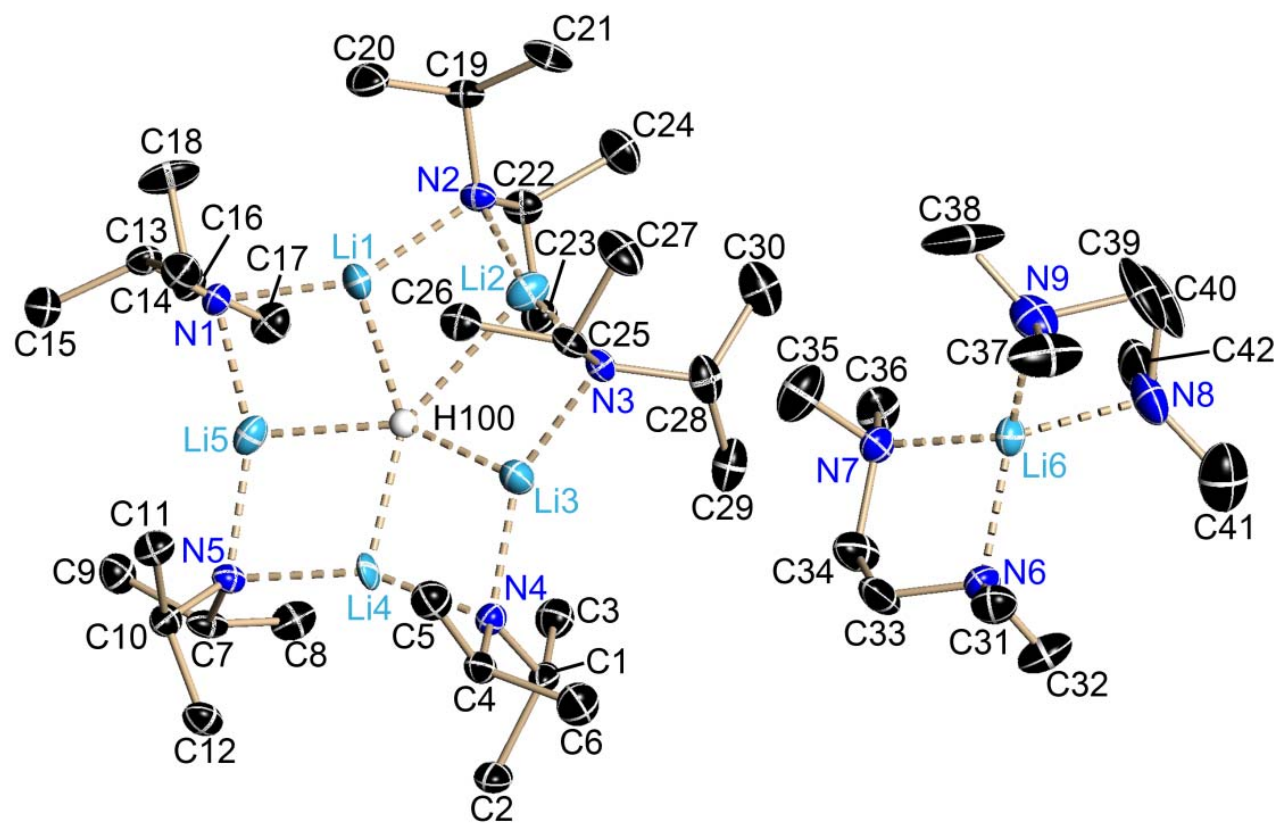

Figure 9-3. Asymmetric unit of Hydrid (3). Hydrogen atoms except for the hydrid anion $\mathrm{H} 100$ are omitted for clarity.

Hydrid (3) crystallizes as colorless, shiver like blocks in the monoclinic space group $P$ n. The hydrid anion $\mathrm{H} 100$ was found in the difference Fourier map and refined without further constraints. Replacement of the hydrid by heavier elements (helium) and modeled positional disorders, where the hydrid anion was partly substitueted by a hydroxide ion yielded considerably worse R-factors. One of the tmeda ligands showed a disorder with occupancy of $36 \%$ (omitted for clarity).

Table 9-3. Crystallographic data for Hydrid(3).

\begin{tabular}{ll|ll} 
identification code & Hydrid & & \\
empirical formula & $\mathrm{C}_{42} \mathrm{H}_{103} \mathrm{Li}_{6} \mathrm{~N}_{9}$ & $\mu\left[\mathrm{mm}^{-1}\right]$ & 0.054 \\
molar mass $\left[\mathrm{g} \mathrm{mol}^{-1}\right]$ & 559.96 & $F(000)$ & 872 \\
crystal size $[\mathrm{mm}]$ & $0.25 \times 0.20 \times 0.20$ & min./max. transmission & $0.9892 / 0.9865$ \\
temperature $[\mathrm{K}]$ & $100(2)$ & $\theta$ range $\left[^{\circ}\right]$ & $1.69-26.02$ \\
crystal system & monoclinic & completeness to $\theta_{\text {max }}$ & $99.9 \%$ \\
space group & $P \mathrm{n}$ & reflections collected & 59361 \\
$a[\AA]]$ & $14.3752(8)$ & independent reflections & 10638 \\
$b[\AA]]$ & $12.8875(7)$ & $R_{\text {int }}$ & 0.0221 \\
$c[\AA]$ & $15.4049(9)$ & restraints / parameters & $2 / 553$
\end{tabular}




\begin{tabular}{ll|ll}
$\alpha\left[^{\circ}\right]$ & 90 & GooF & 1.034 \\
$\beta\left[^{\circ}\right]$ & $108.5250(10)^{\circ}$ & $R 1$ (all data) & 0.0435 \\
$\gamma\left[^{\circ}\right]$ & 90 & $R 1(I>2 \sigma(I))$ & 0.0379 \\
$V\left[\AA^{3}\right]$ & $2706.0(3)$ & $w R 2($ all data) & 0.1070 \\
$\mathrm{Z}$ & 2 & $w R 2(>2 \sigma(I))$ & 0.1005 \\
$\rho_{\text {calc }}\left[\mathrm{g} \mathrm{cm}^{-3}\right]$ & 0.952 & diff. peak/hole $\left[\mathrm{e} \AA^{-3}\right]$ & $0.211 /-0.181$ \\
\hline
\end{tabular}

\subsubsection{Simpkin's amide (4)}

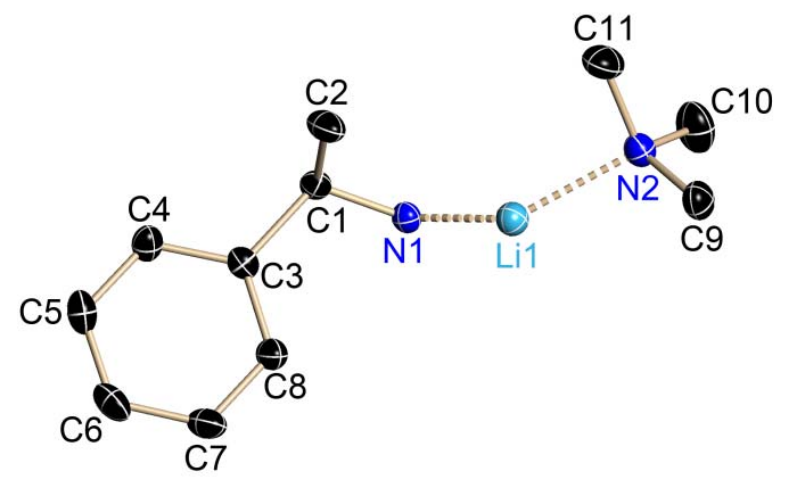

Figure 9-4. Asymmetric unit of Simpkin's Amide (4). Hydrogen atoms are omitted for clarity.

The Simpkin's amide (4) crystallizes as dark red plates in the orthorhombic space group $P 2{ }_{1} 2_{1} 2_{1}$. The asymmetric unit contains only half of the molecule while the other half is generated by crystal symmetry.

\begin{tabular}{|c|c|c|c|}
\hline identification code & simpkins & & \\
\hline empirical formula & $\mathrm{C}_{22} \mathrm{H}_{34} \mathrm{LiN}_{3}$ & $\mu\left[\mathrm{mm}^{-1}\right]$ & 0.060 \\
\hline molar mass $\left[\mathrm{g} \mathrm{mol}^{-1}\right]$ & 347.46 & $F(000)$ & 380 \\
\hline crystal size [mm] & $0.23 \times 0.17 \times 0.10$ & min./max. transmission & $0.99 / 0.834$ \\
\hline temperature $[\mathrm{K}]$ & $100(2)$ & $\theta$ range $\left[{ }^{\circ}\right]$ & $2.00-28.35$ \\
\hline crystal system & Orthorhombic & completeness to $\theta_{\max }$ & $96.0 \%$ \\
\hline space group & $P 2{ }_{1} 2_{1} 2_{1}$ & reflections collected & 7635 \\
\hline$a[\AA]]$ & $12.015(2)$ & independent reflections & 2609 \\
\hline$b[\AA]]$ & $9.0966(18)$ & $R_{\text {int }}$ & 0.0269 \\
\hline$c[\AA]$ & 10.1.7(2) & restraints / parameters & $0 / 121$ \\
\hline$\alpha\left[^{\circ}\right]$ & 90 & GooF & 1.034 \\
\hline$\beta\left[^{\circ}\right]$ & 90 & $R 1$ (all data) & 0.0548 \\
\hline$\gamma\left[^{\circ}\right]$ & 90 & $R 1(1>2 \sigma(I))$ & 0.0394 \\
\hline$V\left[\AA^{3}\right]$ & $1112.3(4)$ & $w R 2$ (all data) & 0.1011 \\
\hline
\end{tabular}




\begin{tabular}{ll|ll}
$\mathrm{Z}$ & 2 & $w R 2(1>2 \sigma(I))$ & 0.0932 \\
$\rho_{\text {calc }}\left[\mathrm{g} \mathrm{cm}^{-3}\right]$ & 1.037 & diff. peak/hole $\left[\mathrm{e} \AA^{-3}\right]$ & $0.202 /-0.149$ \\
\hline
\end{tabular}

\subsection{5 $\mathrm{Me}_{3} \mathrm{ZnLi}$-diglyme (5)}

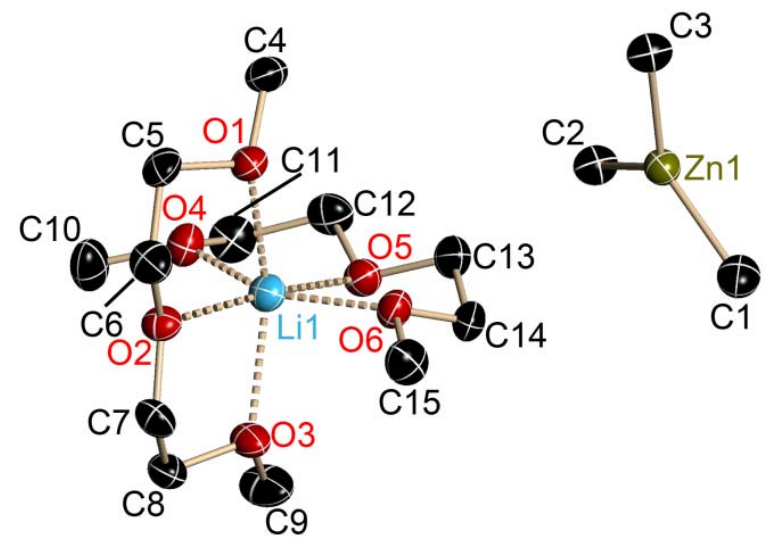

Figure 9-5. Asymmetric unit of $\mathrm{Me}_{3} \mathrm{ZnLi}$-diglyme (5). Hydrogen atoms are omitted for clarity.

The solvent separated complex [(Diglyme $\left.)_{2} \mathrm{Li}\right]\left[\mathrm{ZnMe}_{3}\right](5)$ crystallizes as colorless plates in the monoclinic space group $P 2_{1} / c$.

Table 9-4. Crystallographic data for $\mathrm{Me}_{3} \mathrm{ZnLi}$ diglyme (5).

\begin{tabular}{ll|ll}
\hline identification code & Zincat1 & & \\
empirical formula & $\mathrm{C}_{15} \mathrm{H}_{37} \mathrm{LiO}{ }_{6} \mathrm{Zn}$ & $F(000)$ & 832 \\
molar mass $\left[\mathrm{g} \mathrm{mol}^{-1}\right]$ & 385.76 & min./max. transmission & $0.89 / 0.80$ \\
crystal size $[\mathrm{mm}]$ & $0.20 \times 0.10 \times 0.10$ & $\theta$ range $\left[^{\circ}\right]$ & $1.88-23.81$ \\
temperature $[\mathrm{K}]$ & $100(2)$ & completeness to $\theta_{\max }$ & $99.0 \%$ \\
crystal system & monoclinic & reflections collected & 20157 \\
space group & $P 2_{1} / c$ & independent reflections & 3288 \\
$a[\AA]$ & $11.428(5)$ & $R_{\text {int }}$ & 0.0623 \\
$b[\AA]$ & $11.695(5)$ & restraints/parameters & $0 / 215$ \\
$c[\AA]$ & $1689.7(8) \mathrm{pm}$ & GooF & 1.084 \\
$\beta\left[{ }^{\circ}\right]$ & $108.899(6)$ & $R 1$ (all data) & 0.0495 \\
$V\left[\AA^{3}\right]$ & $2136.5(17)$ & $R 1(>2 \sigma(I))$ & 0.0705 \\
$Z$ & 4 & $w R 2($ all data) & 0.1149 \\
$\rho_{\text {calc }}\left[\mathrm{g} \mathrm{cm}^{-3}\right]$ & 1.199 & $w R 2(/>2 \sigma(I))$ & 0.1233 \\
$\mu\left[\mathrm{mm}^{-1}\right]$ & 1.171 & diff. peak/hole $\left[\mathrm{e} \AA^{-3}\right]$ & $0.680 /-0.535$ \\
\hline
\end{tabular}




\subsection{6 $\mathrm{Me}_{3} \mathrm{ZnLi} \cdot \mathrm{pmdeta}(6)$}

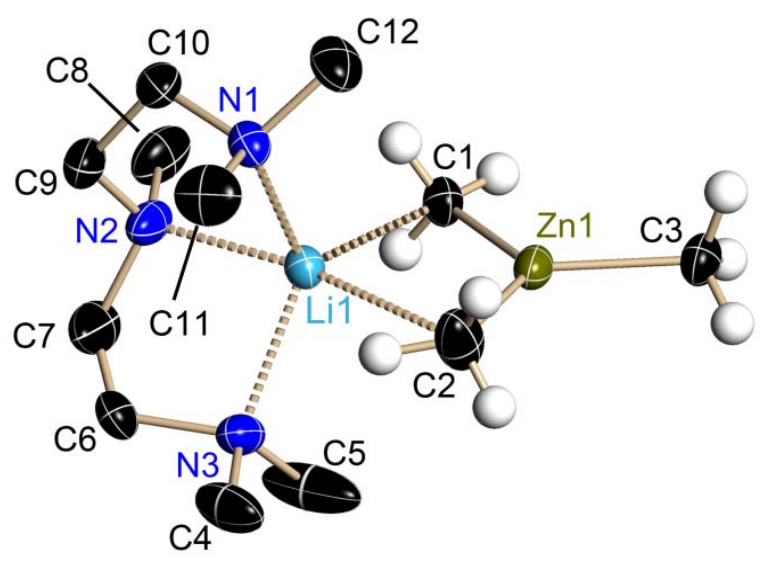

Figure 9-6. Asymmetric unit of $\mathrm{Me}_{3} \mathrm{ZnLi} \cdot$ pmdeta (6). Hydrogen atoms of the donor pmdeta are omitted for clarity.

[(PMDETA)Li( $\mu$-Me $\left.)_{2} Z n M e\right](1)$ crystallizes as colorless needles in the orthorhombic space group Pbca. 
Table 9-5. Crystallographic data for $\mathrm{Me}_{3} \mathrm{ZnLi} \cdot \operatorname{pmdeta}(\mathbf{6})$.

\begin{tabular}{ll|ll}
\hline identification code & Zincat2 & & \\
empirical formula & $\mathrm{C}_{12} \mathrm{H}_{32} \mathrm{LiN}_{3} \mathrm{Zn}$ & $F(000)$ & 1264 \\
molar mass $\left[\mathrm{g} \mathrm{mol}^{-1}\right]$ & 290.72 & min./max. transmission & $0.87 / 0.76$ \\
crystal size $[\mathrm{mm}]$ & $0.20 \times 0.20 \times 0.10$ & $\theta$ range $\left[^{\circ}\right]$ & $2.61-26.37$ \\
temperature $[\mathrm{K}]$ & $100(2)$ & completeness to $\theta_{\max }$ & $99.0 \%$ \\
crystal system & Orthorhombic & reflections collected & 39046 \\
space group & $P b c a$ & independent reflections & 3835 \\
$a[\AA]$ & $12.0040(10)$ & $R_{\text {int }}$ & 0.0317 \\
$b[\AA]$ & $11.3603(10)$ & restraints/parameters & $72 / 208$ \\
$c[\AA]$ & $24.328(2)$ & GooF & 1.048 \\
& & $R 1($ all data $)$ & 0.0370 \\
$V\left[\AA^{3}\right]$ & $3317.5(5)$ & $R 1(/>2 \sigma(I))$ & 0.0292 \\
$Z$ & 8 & $w R 2($ all data) & 0.0715 \\
$\rho_{\text {calc }}\left[\mathrm{g} \mathrm{cm}^{-3}\right]$ & 1.164 & $w R 2(/>2 \sigma(I))$ & 0.0677 \\
$\mu\left[\mathrm{mm}^{-1}\right]$ & 1.466 & diff. peak/hole $\left[\mathrm{e} \AA^{-3}\right]$ & $0.332 /-0.235$ \\
\hline
\end{tabular}

\subsection{7 $\mathrm{Me}_{3} \mathrm{ZnLi} \cdot \mathrm{pmdeta} \cdot \mathrm{LiCl}(7)$}
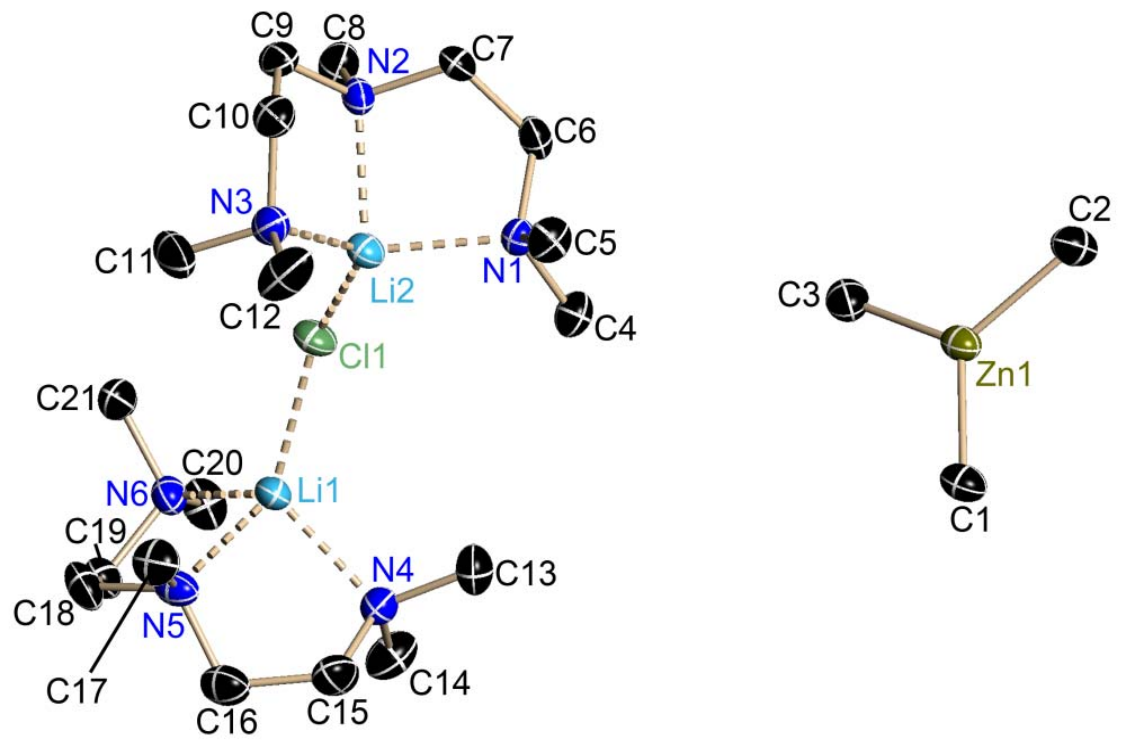

Figure 9-7. Asymmetric unit of $\mathrm{Me}_{3} \mathrm{ZnLi} \cdot p m d e t a \cdot \mathrm{LiCl}(7)$. Hydrogen atoms are omitted for clarity. 
$\mathrm{Me}_{3} \mathrm{ZnLi} \cdot \mathrm{pmdeta} \cdot \mathrm{LiCl}(7)$ crystallizes as colorless crystals in the monoclinic space group $P 2_{1} / n$.

Table 9-6. Crystallographic data for $\mathrm{Me}_{3} \mathrm{ZnLi} \cdot \mathrm{pmdeta} \cdot \mathrm{LiCl}$ (7).

\begin{tabular}{|c|c|c|c|}
\hline identification code & Zincat3 & & \\
\hline empirical formula & $\mathrm{C}_{21} \mathrm{H}_{56} \mathrm{ClL}_{2} \mathrm{i} \mathrm{N}_{6} \mathrm{Zn}$ & $F(000)$ & 1104 \\
\hline molar mass $\left[\mathrm{g} \mathrm{mol}^{-1}\right]$ & 506.42 & min./max. transmission & $0.9141 / 0.9141$ \\
\hline crystal size $[\mathrm{mm}]$ & $0.10 \times 0.10 \times 0.10$ & $\theta$ range $\left[{ }^{\circ}\right]$ & $1.60-26.35$ \\
\hline temperature $[\mathrm{K}]$ & $100(2)$ & completeness to $\theta_{\max }$ & $100 \%$ \\
\hline crystal system & monoclinic & reflections collected & 21295 \\
\hline space group & $P 2_{1} / n$ & independent reflections & 6162 \\
\hline$a[\AA]$ & $8.7649(18)$ & $R_{\text {int }}$ & 0.0385 \\
\hline$b[\AA]]$ & $15.446(3)$ & restraints/parameters & $0 / 293$ \\
\hline \multirow[t]{2}{*}{$c[\AA ̊]$} & $22.656(5)$ & GooF & 1.048 \\
\hline & & $R 1$ (all data) & 0.0646 \\
\hline$V\left[\AA^{3}\right]$ & $3032.1(11)$ & $R 1(I>2 \sigma(I))$ & 0.0394 \\
\hline Z & 4 & $w R 2$ (all data) & 0.0951 \\
\hline$\rho_{\text {calc }}\left[\mathrm{g} \mathrm{cm}^{-3}\right]$ & 1.109 & $w R 2(1>2 \sigma(I))$ & 0.0885 \\
\hline$\mu\left[\mathrm{mm}^{-1}\right]$ & 0.915 & diff. peak/hole $\left[e \AA^{-3}\right]$ & $0.563 /-0.365$ \\
\hline
\end{tabular}




\subsection{Cooperation Projects}

Several service structures were determined in cooperation with the research group of Prof. Dr. H. W. Roesky. In addition, for two compounds (14 and 15), in collaboration with J. Henn computational studies including a topological analysis according to Bader's QTAIM have been performed. The synthetic chemists were Dr. R. Ghadwal and Dr. J. Li.

\subsubsection{NHC. $\mathrm{SiCl}_{2} \cdot(14)$}

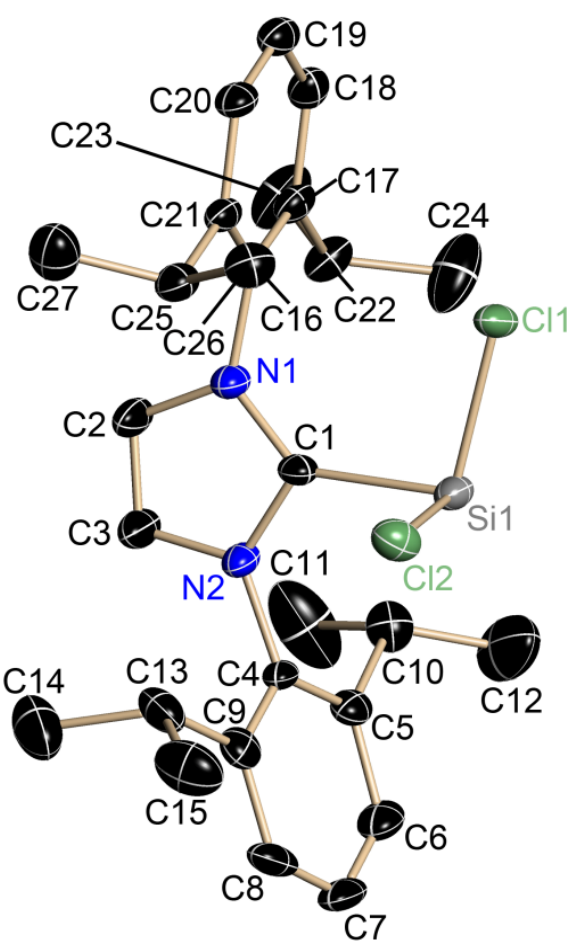

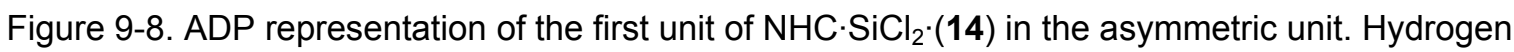
atoms are omitted for clarity.

$\mathrm{NHC} \cdot \mathrm{SiCl}_{2}(14)$ is the first Lewis base stabilized dichlorosilylene. It crystallises as orange plates in the monoclinic space group $P 2_{1} / c$ with two independent molecules in the asymmetric unit. All bond lengths and angles are given as average numbers of the two molecules. 
Table 9-7. Crystallographic data for $\mathrm{NHC} \cdot \mathrm{SiCl}_{2} \cdot(\mathbf{1 4})$.

\begin{tabular}{ll|ll}
\hline identification code & Rajendra3 & & \\
empirical formula & $\mathrm{C}_{27} \mathrm{H}_{36} \mathrm{Cl}_{2} \mathrm{~N}_{2} \mathrm{Si}$ & $\mu\left[\mathrm{mm}^{-1}\right]$ & 0.298 \\
molar mass $\left[\mathrm{g} \mathrm{mol}^{-1}\right]$ & 487.57 & $F(000)$ & 2080 \\
crystal size $[\mathrm{mm}]$ & $0.32 \times 0.15 \times 0.10$ & min./max. transmission & $0.9107 / 0.9708$ \\
temperature $[\mathrm{K}]$ & $100(2)$ & $\theta$ range $\left[^{\circ}\right]$ & $1.36-25.39$ \\
crystal system & $P 2_{1} / c$ & completeness to $\theta_{\text {max }}$ & $99.6 \%$ \\
space group & monoclinic & reflections collected & 55762 \\
$a[\AA]$ & $16.135(4)$ & independent reflections & 10029 \\
$b[\AA]$ & $19.150(5)$ & $R_{\text {int }}$ & 0.0988 \\
$c[\AA]$ & $19.068(5)$ & restraints/parameters & $0 / 594$ \\
$\alpha\left[^{\circ}\right]$ & 90 & GooF & 1.027 \\
$\beta\left[^{\circ}\right]$ & $111.679(4)$ & $R 1$ (all data) & 0.0978 \\
$\gamma\left[^{\circ}\right]$ & 90 & $R 1(>2 \sigma(I))$ & 0.0407 \\
$V\left[\AA^{3}\right]$ & $5475(3)$ & $w R 2($ all data) & 0.1680 \\
$Z$ & 8 & $w R 2(/>2 \sigma(I))$ & 0.0991 \\
$\rho_{\text {calc }}\left[\mathrm{g} \mathrm{cm}{ }^{-3}\right]$ & 1.183 & diff. peak/hole $\left[\mathrm{e} \AA^{-3}\right]$ & $0.763 /-0.407$ \\
\hline
\end{tabular}

In addition, computational studies starting from the crystal coordinates have been performed. After a gas phase optimization on the B3-P86/TZVP level of theory, $\rho(\mathbf{r})$ and $\nabla^{2} \rho(\mathbf{r})$ have been calculated on a grid (mesh size 0.1 Bohr) with DGRID. Of special interest was the localization of the silylenic lone pair. In the Laplacian of $\rho(\mathbf{r})$, four VSCCs near Si were found and one of them in the non-bonding region can be interpreted within QTAIM as marking a lone pair density $\mathrm{LP}_{\mathrm{Si}}$. The angle between $\mathrm{LP}_{\mathrm{Si}}-\mathrm{Si}-\mathrm{C}$ (carbene), LP $\mathrm{P}_{\mathrm{Si}}-\mathrm{Si}-$ $\mathrm{Cl}$ (inplane) and $\mathrm{LP}_{\mathrm{Si}}-\mathrm{Si}-\mathrm{Cl}$ (orth.plane) are $114.40^{\circ}, 121.93^{\circ}$ and $123.37^{\circ}$, respectively. This arrangement can be visualized by an isosurface representation of $\nabla^{2} \rho(r)$ at -0.53 e $\AA^{-5}$ (figure 9-9). 


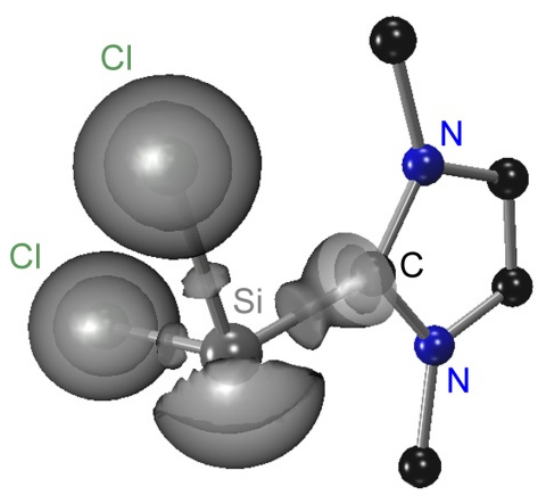

a

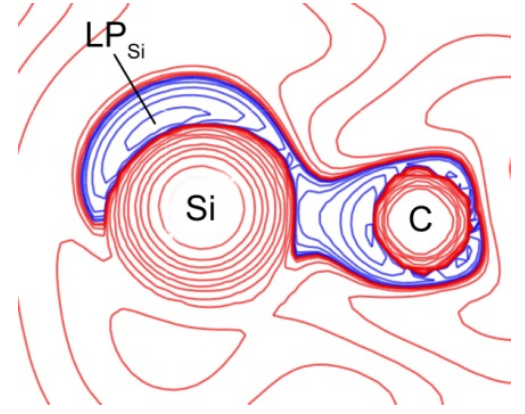

b

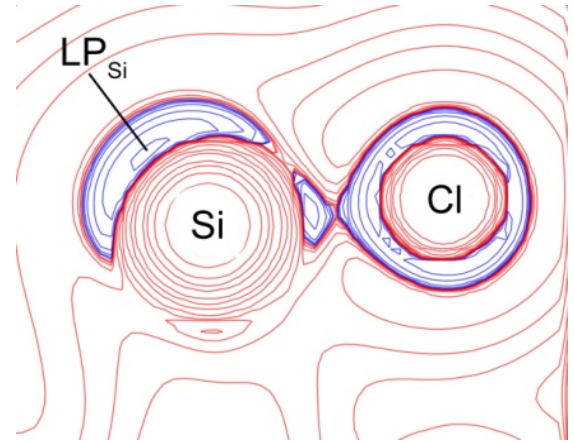

C

Figure 9-9. Isosurface plot of $\nabla^{2} \rho(\mathbf{r})$ of 14 at the $-0.53 \mathrm{e} / \AA^{5}$ level around $\mathrm{Si}(\mathbf{a})$, contour plots of $-\nabla^{2} \rho(\mathbf{r})$ in the C-Si-VSCC plane (b) and in the Cl(inplane)-Si-VSCC (c). Local charge concentrations are depicted in blue, charge depletions in red. The contour values are at $0.2 \cdot 10^{\mathrm{n}}, 0.4 \cdot 10^{\mathrm{n}}$ and $0.8 \cdot 10^{\mathrm{n}}$ with $n=-3, \pm 2, \pm 1$.

In agreement the highest occupied orbital (HOMO) shows the most distinct contribution from the silicium atom (figure 9-10), while the lowest occupied orbital (LUMO) is mainly located at the carbene ligand (figure 9-11).
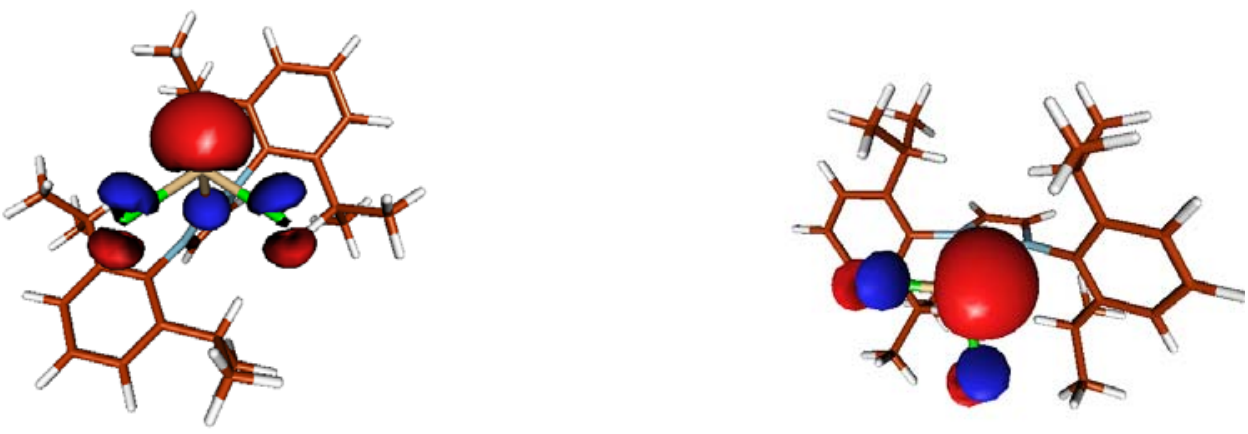

Figure 9-10. Two orientations of the HOMO of the optimized singlet of Silylene (14). 

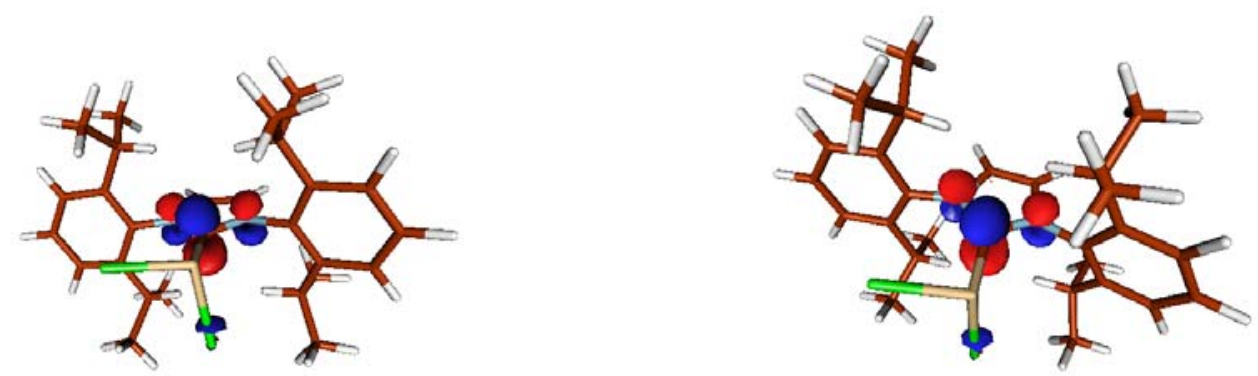

Figure 9-11. Two orientations of the LUMO of the optimized singlet of Silylene (14).

\subsection{2 $\mathrm{NHC} \cdot \mathrm{SiCl}_{2} \cdot \mathrm{B}\left(\mathrm{C}_{6} \mathrm{~F}_{5}\right)_{3}(15)$}

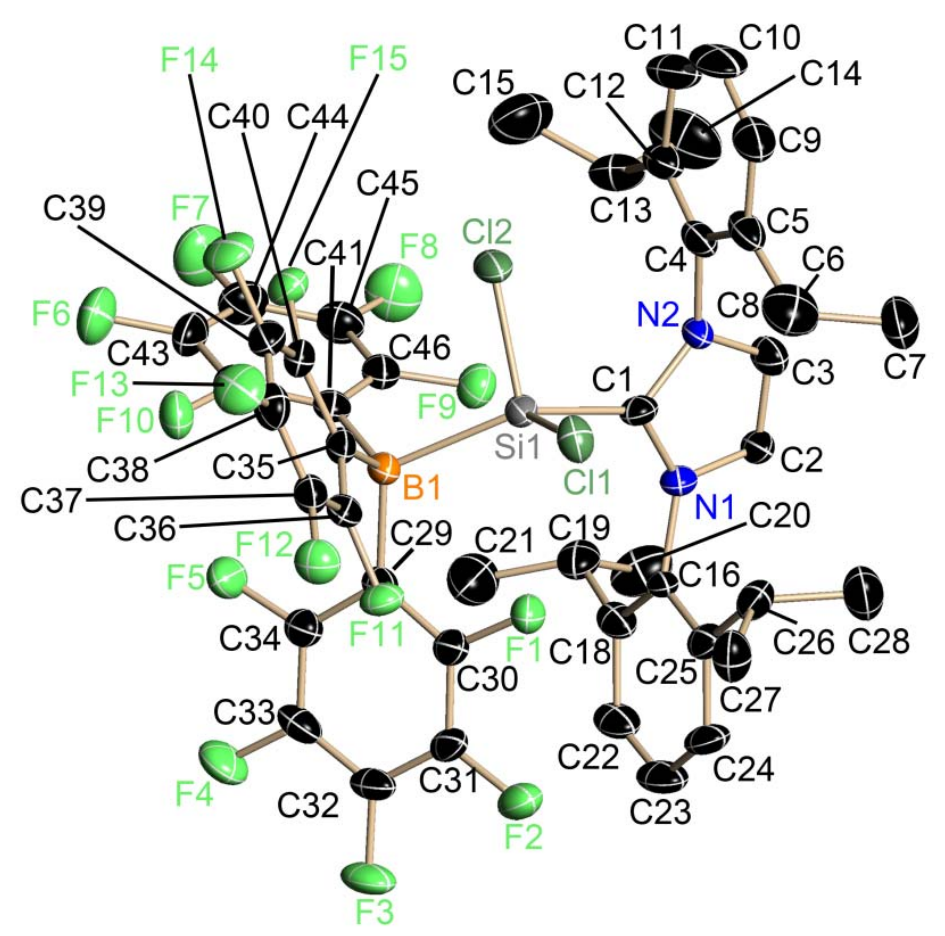

Figure 9-12. Asymmetric unit of $\mathrm{NHC} \cdot \mathrm{SiCl}_{2} \cdot \mathrm{B}\left(\mathrm{C}_{6} \mathrm{~F}_{5}\right)_{3}(15)$. Hydrogen atoms are omitted for clarity.

$\mathrm{NHC} \cdot \mathrm{SiCl}_{2} \cdot \mathrm{B}\left(\mathrm{C}_{6} \mathrm{~F}_{5}\right)(15)$ is the silylene-borane adduct being formed by the reaction of the Lewis base stabilized $\mathrm{NHC} \cdot \mathrm{SiCl}_{2}$ (14) with the Lewis acid $\mathrm{B}\left(\mathrm{C}_{6} \mathrm{~F}_{5}\right)_{3}$. 15 crystallizes as colorless plates in the triclinic space group $P_{1}^{-}$as a twin with three toluene molecules in the crystal lattice. 
Table 9-8. Crystallographic data for $\mathrm{NHC} \cdot \mathrm{SiCl}_{2} \cdot \mathrm{B}\left(\mathrm{C}_{6} \mathrm{~F}_{5}\right)_{3}(\mathbf{1 5})$.

\begin{tabular}{|c|c|c|c|}
\hline $\begin{array}{l}\text { identification code } \\
\text { empirical formula }\end{array}$ & $\begin{array}{l}\quad \text { Rajendra12 } \\
\left(\mathrm{C}_{45} \mathrm{H}_{36} \mathrm{BCl}_{2} \mathrm{~F}_{15} \mathrm{~N}_{2} \mathrm{Si}\right) \\
\text { (toluene })_{3}\end{array}$ & $\mu\left[\mathrm{mm}^{-1}\right]$ & 0.240 \\
\hline molar mass $\left[\mathrm{g} \mathrm{mol}^{-1}\right]$ & 2275.52 & $F(000)$ & 2332 \\
\hline crystal size $[\mathrm{mm}]$ & $0.20 \times 0.15 \times 0.05$ & min./max. transmission & $0.9881 / 0.9537$ \\
\hline temperature $[\mathrm{K}]$ & $100(5)$ & $\theta$ range $\left[{ }^{\circ}\right]$ & $2.35-25.45$ \\
\hline crystal system & triclinic & completeness to $\theta_{\max }$ & $98.7 \%$ \\
\hline space group & $P_{1}^{-}$ & reflections collected & 84398 \\
\hline$a[\AA]$ & $11.615(2)$ & independent reflections & 19865 \\
\hline$b[\AA]]$ & $21.069(4)$ & $R_{\text {int }}$ & 0.0634 \\
\hline$c[\AA]$ & $22.573(4)$ & restraints / parameters & $183 / 1398$ \\
\hline$\alpha\left[^{\circ}\right]$ & $75.772(2)$ & GooF & 1.101 \\
\hline$\beta\left[^{\circ}\right]$ & $79.651(2)$ & $R 1$ (all data) & 0.0953 \\
\hline$\gamma\left[^{\circ}\right]$ & $89.560(2)$ & $R 1(\mid>2 \sigma(I))$ & 0.0737 \\
\hline$V\left[\AA^{3}\right]$ & $5263.7(15)$ & $w R 2$ (all data) & 0.1846 \\
\hline Z & 2 & $w R 2(1>2 \sigma(l))$ & 0.1725 \\
\hline$\rho_{\text {calc }}\left[\mathrm{g} \mathrm{cm}^{-3}\right]$ & 1.436 & diff. peak/hole $\left[e \AA^{-3}\right]$ & $0.803 /-0.558$ \\
\hline
\end{tabular}

Remarkably, in $15 \mathrm{SiCl}_{2}$ acts simultaneously as Lewis base and acid. On the one hand, 15 exhibits a $\mathrm{C} \rightarrow \mathrm{Si}$ donor bond from the NHC ligand to the $\mathrm{Si} 1$ atom, which stabilizes the dichlorosilylene. On the other hand, the silylene forms a $\mathrm{Si} \rightarrow \mathrm{B}$ donor bond to the electron deficient $\mathrm{B} 1$ atom. To investigate these bonding modes, based on the crystal coordinates a gas phase optimization on the B3LYP/TZVP level of theory was performed. Thereon, the electron density (ED) distribution was analyzed in terms of Bader's QTAIM theory. Both $\mathrm{C} \rightarrow \mathrm{Si}$ and $\mathrm{Si} \rightarrow \mathrm{B}$ donor bonds were confirmed by bond critical points (BCPs) and their characteristics deduced by evaluating $\rho\left(\mathbf{r}_{\mathrm{BCP}}\right)$ and $\nabla^{2} \rho\left(\mathbf{r}_{\mathrm{BCP}}\right) . \rho(\mathbf{r})$. The electron density at the critical points is in both cases nearly identical $\left(0.55\right.$ and $\left.0.57 \mathrm{e}^{-3}\right)$, so from this side no distinction can be made. However, the $\nabla^{2} \rho\left(\mathrm{r}_{\mathrm{BCP}}\right)$ reveals a positive value of $2.67 \mathrm{e} \AA^{-5}$ for the carbon-silicon bond, but a negative value of $-2.66 \mathrm{e}^{-5}$ for the silicon-boron bond. Figure 913 illustrates that the first BCP resides in a region of charge depletion, while the second is located in a region of charge accumulation. This indicates that the $\mathrm{Si} \rightarrow \mathrm{B}$ bond features a stronger covalent character compared to the $\mathrm{C} \rightarrow \mathrm{Si}$ bond. 

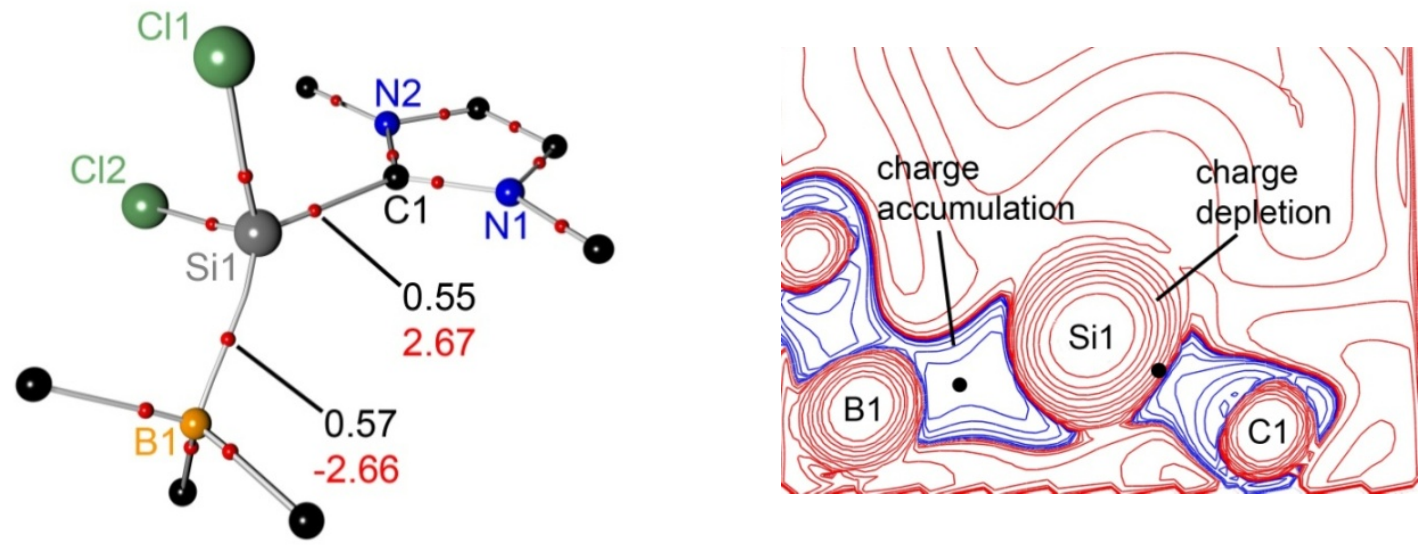

Figure 9-13. Molecular graph of 15 (left) obtained from a gas phase optimization (B3LYP/TZVP). The small red spheres indicate BCPs, with $\rho\left(\mathbf{r}_{\mathrm{BCP}}\right)\left[\mathrm{e} \AA^{-3}\right]$ (upper number) and $\nabla^{2} \rho\left(\mathbf{r}_{\mathrm{BCP}}\right)\left[\mathrm{e} \AA^{-5}\right]$ (bottom number) and contour plot of $\nabla^{2} \rho(\mathbf{r})$ (right) for the C1-Si1-B1 plane.

Since the BCP between $\mathrm{C} 1$ and $\mathrm{Si} 1$ is close to a region, where the Laplacian changes sign, meaning that just a slight inaccuracy in its determination can lead to considerable deviations, $\nabla^{2} \rho(\mathbf{r})$ was monitored along the whole bond path, too. While the Laplacian along the bond path between the carbon and the silicon atom is considerably negative in the carbon basin and only changes sign close to the BCP (figure 9-14, left) the related function along the $\mathrm{Si} \rightarrow \mathrm{B}$ bond (figure 9-14, right) shows much more balanced behavior, indicative for a covalent bond. These QTAIM results are in accordance with the differences in electronegativity. $\mathrm{EN}_{\mathrm{Si}}$ is 0.8 smaller than that of carbon, but only 0.3 smaller than of boron.
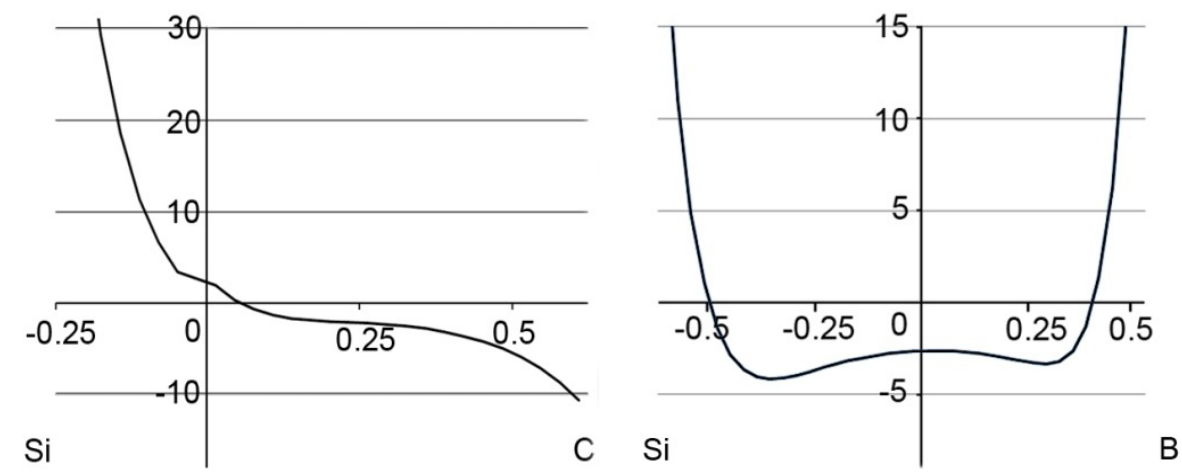

Figure 9-14. $\nabla^{2} \rho(\mathbf{r})\left[\mathrm{e} \AA^{-5}\right]$ of the Si1-C1-bond (left) and along the bond path Si1-B1 (right), $[\AA]$ $(0=\mathrm{BCP})$. 


\subsubsection{NHC. $\left(\mathrm{C}_{14} \mathrm{H}_{10} \mathrm{Si}_{3} \mathrm{Cl}_{6}\right)$}

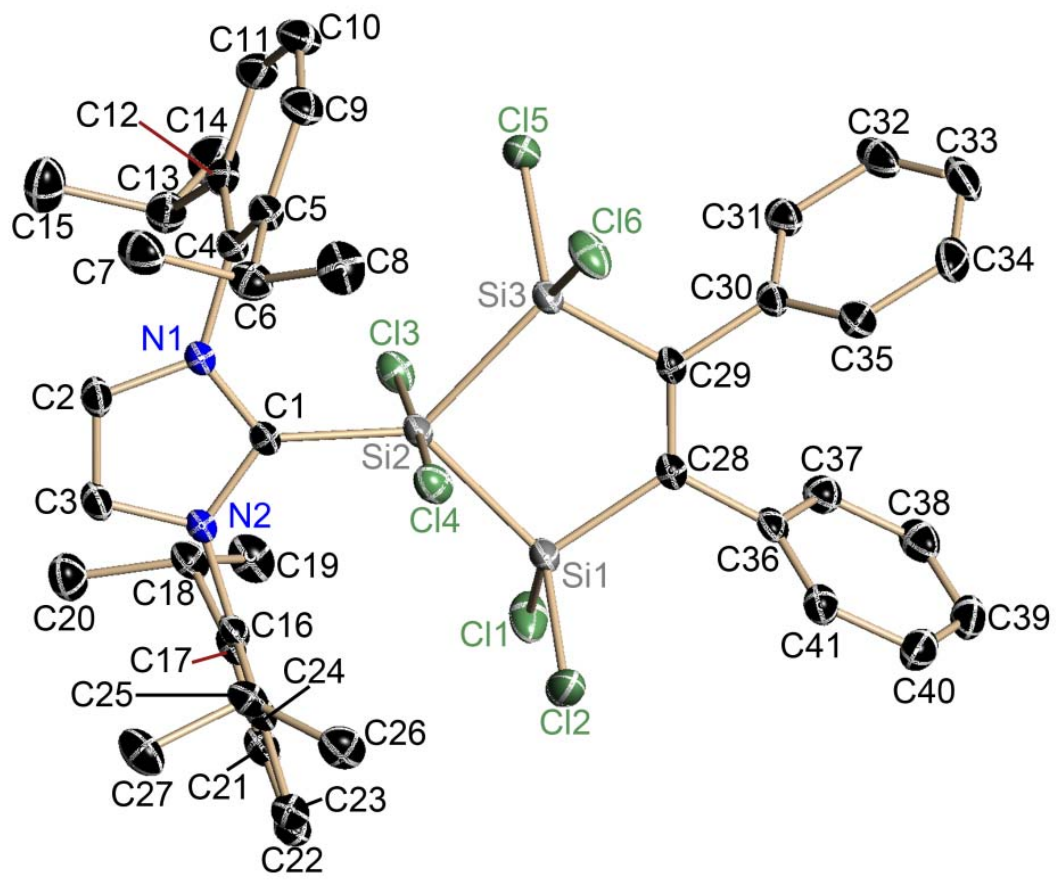

Figure 9-15. Asymmetric unit of $\mathrm{NHC} \cdot\left(\mathrm{C}_{14} \mathrm{H}_{10} \mathrm{Si}_{3} \mathrm{Cl}_{6}\right)$. Hydrogen atoms and solvent molecules are omitted for clarity.

$\mathrm{NHC} \cdot\left(\mathrm{C}_{14} \mathrm{H}_{10} \mathrm{Si}_{3} \mathrm{Cl}_{6}\right)$ crystallizes as colorless plates in the triclinic space group $P \overline{1}$ with toluene as lattice solvent. It contains three $\mathrm{SiCl}_{2}$ moieties in a five-membered $\left(\mathrm{Si}_{3} \mathrm{C}_{2}\right)$ ring. One toluene molecule displays a disorder around an inversion center, which was modeled by rigid body constraints (FRAG and FEND) and restraints on the displacement parameters (SIMU, DELU). 
Table 9-9. Crystallographic data for $\mathrm{NHC} \cdot\left(\mathrm{C}_{14} \mathrm{H}_{10} \mathrm{Si}_{3} \mathrm{Cl}_{6}\right)$.

\begin{tabular}{ll|ll}
\hline identification code & Rajendra4 & & \\
empirical formula & $\mathrm{C}_{58.50} \mathrm{H}_{66} \mathrm{Cl}_{6} \mathrm{~N}_{2} \mathrm{Si}_{3}$ & $\mu\left[\mathrm{mm}^{-1}\right]$ & 0.401 \\
molar mass $\left[\mathrm{g} \mathrm{mol}{ }^{-1}\right]$ & 1094.10 & $F(000)$ & 1150 \\
crystal size $[\mathrm{mm}]$ & $0.39 \times 0.17 \times 0.05$ & min./max. transmission & $0.8592 / 0.9802$ \\
temperature $[\mathrm{K}]$ & $100(2)$ & $\theta$ range $\left[^{\circ}\right]$ & $1.76-26.73$ \\
crystal system & triclinic & completeness to $\theta_{\max }$ & $99.8 \%$ \\
space group & $P \overline{1}$ & reflections collected & 62828 \\
$a[\AA]$ & $10.8505(10)$ & independent reflections & 12152 \\
$b[\AA]$ & $11.7674(10)$ & $R_{\text {int }}$ & 0.0355 \\
$c[\AA]$ & $22.944(2)$ & restraints / parameters & $57 / 654$ \\
$\alpha\left[^{\circ}\right]$ & $89.9000(10)$ & GooF & 1.037 \\
$\beta\left[^{\circ}\right]$ & $82.9020(10)$ & $R 1($ all data) & 0.0505 \\
$\gamma\left[^{\circ}\right]$ & $80.4590(10)$ & $R 1(>2 \sigma(I))$ & 0.0361 \\
$V\left[\AA^{3}\right]$ & $2866.3(4)$ & $w R 2($ all data) & 0.0884 \\
$Z$ & 2 & $w R 2(/>2 \sigma(I))$ & 0.0823 \\
$\rho_{\text {calc }}\left[\mathrm{g} \mathrm{cm}{ }^{-3}\right]$ & 1.268 & diff. peak/hole $\left[\mathrm{e} \AA^{-3}\right]$ & $0.435 /-0.403$ \\
\hline
\end{tabular}




\subsubsection{NHC.SiF 4}

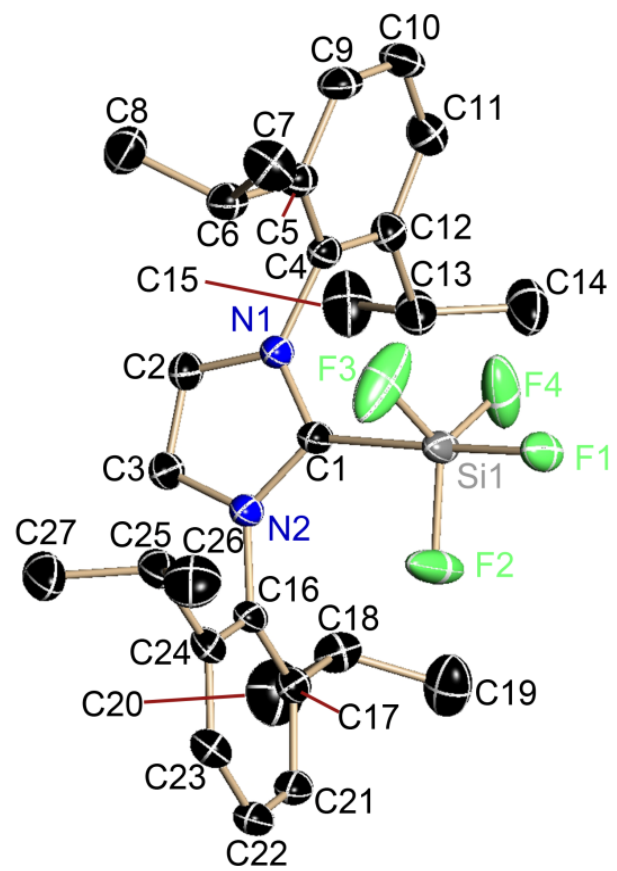

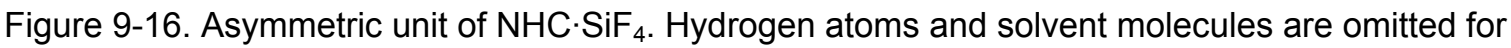
clarity.

$\mathrm{NHC} \cdot \mathrm{SiF}_{4}$. crystallizes as colorless plates in the monoclinic space group $c 2 / c$ with free toluene as lattice solvent.

Table 9-10. Crystallographic data for $\mathrm{NHC} \cdot \mathrm{SiF}_{4}$.

\begin{tabular}{ll|ll} 
identification code & Rajendra5 & & \\
empirical formula & $\mathrm{C}_{37.50} \mathrm{H}_{48} \mathrm{~F}_{4} \mathrm{~N}_{2} \mathrm{Si}$ & $\mu\left[\mathrm{mm}^{-1}\right]$ & 0.112 \\
molar mass $\left[\mathrm{g} \mathrm{mol}{ }^{-1}\right]$ & 630.87 & $F(000)$ & 2696 \\
crystal size $[\mathrm{mm}]$ & $0.55 \times 0.25 \times 0.01$ & min./max. transmission & $0.9989 / 0.9460$ \\
temperature $[\mathrm{K}]$ & $100(2)$ & $\theta$ range $\left[^{\circ}\right]$ & $1.72-26.55$ \\
crystal system & monoclinic & completeness to $\theta_{\text {max }}$ & $99.8 \%$ \\
space group & $C 2 / c$ & reflections collected & 76619 \\
$a[\AA]$ & $22.332(5)$ & independent reflections & 7534 \\
$b[\AA]$ & $14.576(4)$ & $R$ int & 0.0435 \\
$c[\AA]$ & $24.406(8)$ & restraints / parameters & $57 / 431$ \\
$\alpha\left[^{\circ}\right]$ & 90 & GooF & 1.038 \\
$\beta\left[^{\circ}\right]$ & $R 1$ (all data) & 0.0744 \\
$\gamma\left[^{\circ}\right]$ & $114.188(3)$ & $R 1(>2 \sigma(I))$ & 0.0526 \\
$V\left[\AA^{3}\right]$ & 90 & $w R 2$ (all data) & 0.1467
\end{tabular}




\begin{tabular}{ll|ll}
$\mathrm{Z}$ & 8 & $w R 2(>2 \sigma(I))$ & 0.1313 \\
$\rho_{\text {calc }}\left[\mathrm{g} \mathrm{cm}^{-3}\right]$ & 1.156 & diff. peak/hole $\left[\mathrm{e} \AA^{-3}\right]$ & $0.836 /-0.518$ \\
\hline
\end{tabular}

\subsubsection{NHC.SiBr 4}

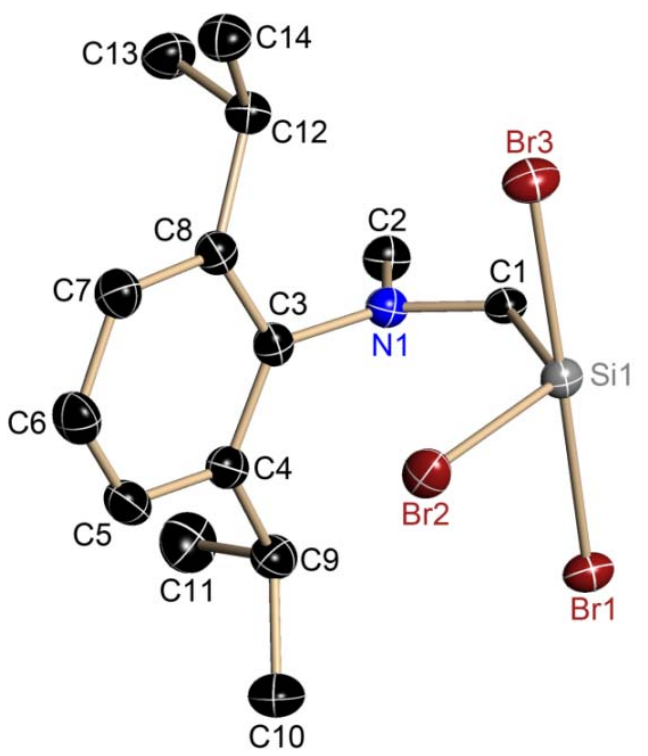

Figure 9-17. Asymmetric unit of $\mathrm{NHC} \cdot \mathrm{SiBr}_{4}$. Hydrogen atoms and solvent molecules are omitted for clarity.

$\mathrm{NHC} \cdot \mathrm{SiBr}_{4}$. crystallizes as colorless blocks in the orthorhombic space group Pnma with free toluene as lattice solvent.

Table 9-11. Crystallographic data for $\mathrm{NHC} \cdot \mathrm{SiBr}_{4}$.

\begin{tabular}{ll|ll} 
identification code & Rajendra6 & & \\
empirical formula & $\mathrm{C}_{41} \mathrm{H}_{52} \mathrm{Br}_{4} \mathrm{~N}_{2} \mathrm{Si}$ & $\mu\left[\mathrm{mm}^{-1}\right]$ & 3.966 \\
molar mass $\left[\mathrm{g} \mathrm{mol}{ }^{-1}\right]$ & 920.58 & $F(000)$ & 1864 \\
crystal size $[\mathrm{mm}]$ & $0.40 \times 0.30 \times 0.15$ & min./max. transmission & $0.9892 / 0.9755$ \\
temperature $[\mathrm{K}]$ & $99(2)$ & $\theta$ range $\left[^{\circ}\right]$ & $1.06-27.12$ \\
crystal system & orthorhombic & completeness to $\theta_{\max }$ & $99.0 \%$ \\
space group & $P n m a$ & reflections collected & 79555 \\
$a[\AA]$ & $16.0120(16)$ & independent reflections & 15220 \\
$b[\AA]]$ & $14.9509(15)$ & $R_{\text {int }}$ & $0.0448]$ \\
$c[\AA]$ & $17.2127(17)$ & restraints / parameters & $0 / 244$ \\
$\alpha\left[^{\circ}\right]$ & 90 & GooF & 1.070 \\
$\beta\left[^{\circ}\right]$ & 90 & $R 1($ all data $)$ & 0.0292 \\
$\gamma\left[^{\circ}\right]$ & 90 & $R 1(>2 \sigma(I))$ & 0.0232
\end{tabular}




\begin{tabular}{ll|ll}
$V\left[\AA^{3}\right]$ & $4120.6(7)$ & $w R 2$ (all data) & 0.0617 \\
$Z$ & 4 & $w R 2(/>2 \sigma(I))$ & 0.0587 \\
$\rho_{\text {calc }}\left[\mathrm{g} \mathrm{cm}^{-3}\right]$ & 1.484 & diff. peak/hole $\left[\mathrm{e} \AA^{-3}\right]$ & $0.403 /-0.320$ \\
\hline
\end{tabular}




\subsection{6 $(\mathrm{NHC})_{2} \cdot \mathrm{SiF}_{4}$}

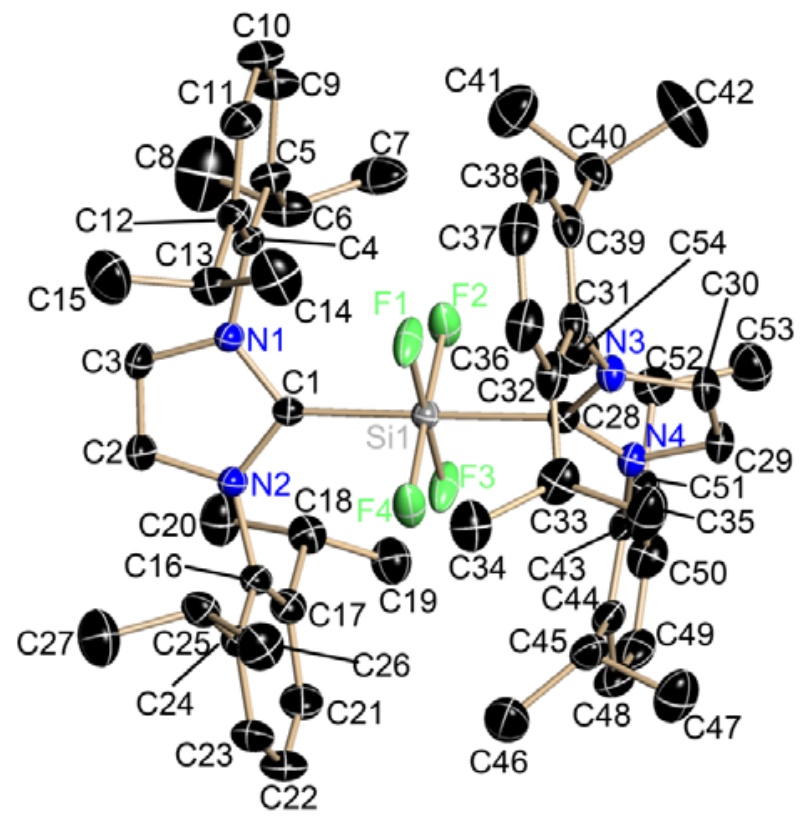

Figure 9-18. Asymmetric unit of $\left(\mathrm{NHC}_{2} \cdot \mathrm{SiF}_{4}\right.$. Hydrogen atoms and solvent molecules are omitted for clarity.

Table 9-12. Crystallographic data for $\left(\mathrm{NHC}_{2} \cdot \mathrm{SiF}_{4}\right.$.

\begin{tabular}{ll|ll}
\hline identification code & Rajendra8 & & \\
empirical formula & $\mathrm{C}_{61} \mathrm{H}_{80} \mathrm{~F}_{4} \mathrm{~N}_{4} \mathrm{Si}$ & $\mu\left[\mathrm{mm}^{-1}\right]$ & 0.078 \\
molar mass $\left[\mathrm{g} \mathrm{mol}^{-1}\right]$ & 937.38 & $F(000)$ & 1048 \\
crystal size $[\mathrm{mm}]$ & $0.32 \times 0.14 \times 0.14$ & min./max. transmission & $0.9892 / 0.9755$ \\
temperature $[\mathrm{K}]$ & $100(2)$ & $\theta$ range $\left[^{\circ}\right]$ & 1.06 to $27.12^{\circ}$ \\
crystal system & triclinic & completeness to $\theta_{\text {max }}$ & $99.7 \%$ \\
space group & $P \mathrm{I}$ & reflections collected & 79555 \\
$a[\AA]$ & $12.7184(11)$ & independent reflections & 15220 \\
$b[\AA]$ & $14.6505(11)$ & $R_{\text {int }}$ & $0.0448]$ \\
$c[\AA]$ & $20.0103(16)$ & restraints / parameters & $48 / 685$ \\
$\alpha\left[^{\circ}\right]$ & $73.5780(10)$ & GooF & 1.052 \\
$\beta\left[^{\circ}\right]$ & $85.8090(10)$ & $R 1$ (all data) & 0.0713 \\
$\gamma\left[^{\circ}\right]$ & $74.8090(10)$ & $R 1(>2 \sigma(I))$ & 0.0520 \\
$V\left[\AA^{3}\right]$ & $3451.5(5)$ & $w R 2$ (all data) & 0.1362 \\
$Z$ & 2 & $w R 2(>2 \sigma(I))$ & 0.1293 \\
$\rho_{\text {calc }}\left[\mathrm{g} \mathrm{cm}{ }^{-3}\right]$ & 0.937 & diff. peak/hole $\left[\mathrm{e} \AA^{-3}\right]$ & $0.403 /-0.320$ \\
\hline
\end{tabular}


$(\mathrm{NHC})_{2} \cdot \mathrm{SiF}_{4}$. crystallizes as colorless plates in the triclinic space group PI. The crystals displayed severe twinning and a disorder of the fluorine molecules. In addition, toluene as lattice solvent is present. One toluene molecules could be modeled successfully, while diffuse residual density of a further solvent molecule was substracted out by the program SQUEEZE of the PLATON suite by A. L. Spek. ${ }^{[203]}$

\subsection{7 $\left[\left(\mathrm{NHC} \cdot \mathrm{SiCl}_{2}\right)_{2} \cdot \mathrm{Co}(\mathrm{CO})_{3}\right]\left[\mathrm{CoCl}_{3}(\right.$ thf $\left.)\right]$}

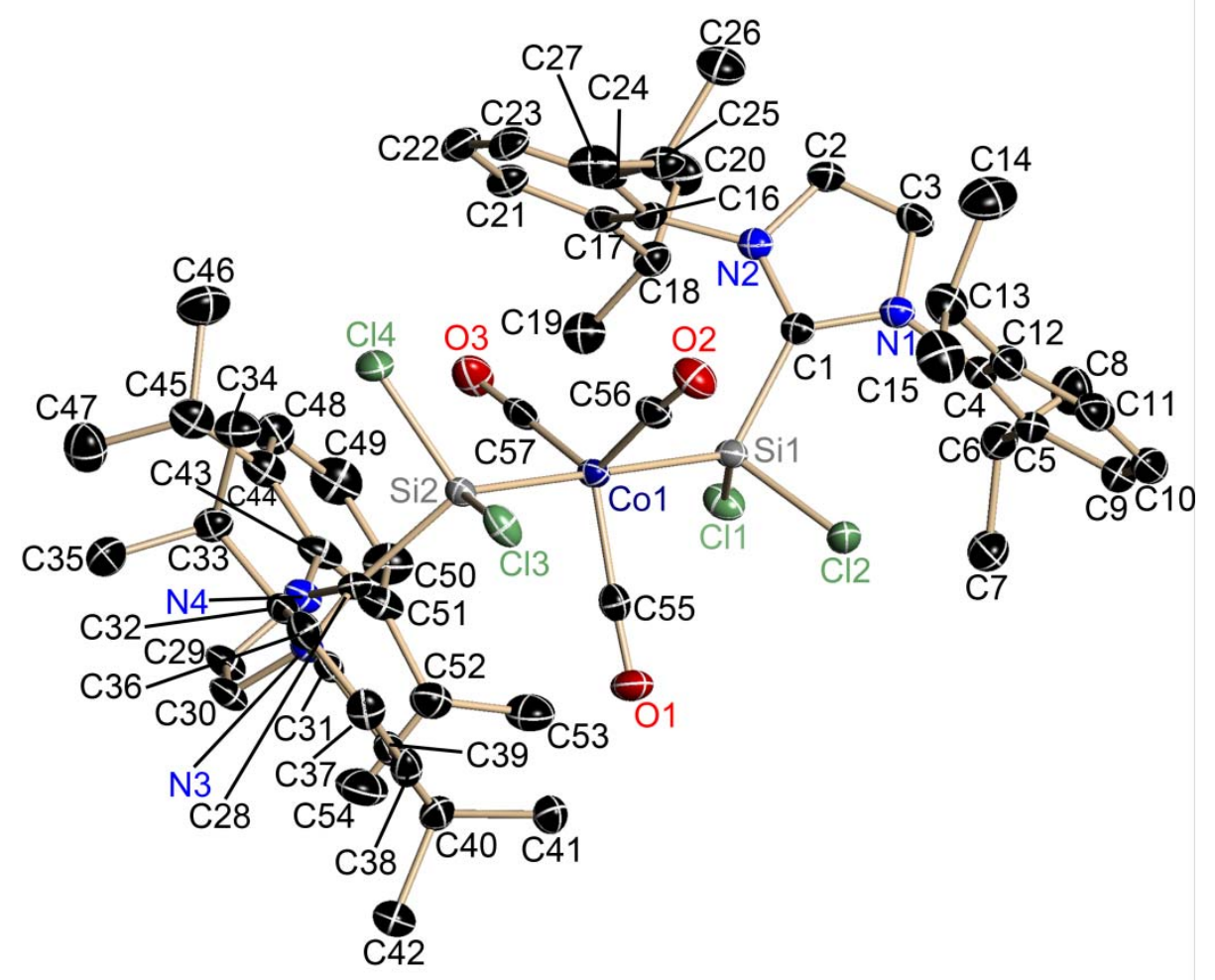

Figure 9-19. ADP representation of $\left[\left(\mathrm{NHC} \cdot \mathrm{SiCl}_{2}\right)_{2} \mathrm{Co}(\mathrm{CO})_{3}\right.$ in the asymmetric unit. Hydrogen atoms, counter ion and solvent molecules are omitted for clarity.

$\left[\left(\mathrm{NHC} \cdot \mathrm{SiCl}_{2}\right)_{2} \cdot \mathrm{Co}(\mathrm{CO})_{3}\right]\left[\mathrm{CoCl}_{3}\right.$ (thf) $]$ crystallizes as blue plates in the monoclinic space group $P 2_{1} / n$. The crystal is twinned and features two domains. Furthermore, besides the a disorder in the $\left[\mathrm{CoCl}_{3}\right.$ (thf)] counter ion additionaly three disordered thf molecules are present in the asymmetric unit, which were modeled with rigid group constraints (FRAG/FEND) for database known thf moieties, and restraints on displacement parameters (SIMU, DELU, ISOR). 
Table 9-13. Crystallographic data for $\left[\left(\mathrm{NHC} \cdot \mathrm{SiCl}_{2}\right)_{2} \cdot \mathrm{Co}(\mathrm{CO})_{3}\right.$.

\begin{tabular}{|c|c|c|c|}
\hline identification code & Li3-Co & & \\
\hline empirical formula & $\mathrm{C}_{69} \mathrm{H}_{96} \mathrm{C}_{17} \mathrm{Co}_{2} \mathrm{~N}_{4} \mathrm{O}_{6} \mathrm{Si}_{2}$ & $\mu\left[\mathrm{mm}^{-1}\right]$ & 0.771 \\
\hline molar mass $\left[\mathrm{g} \mathrm{mol}^{-1}\right]$ & 1499.69 & $F(000)$ & 3148 \\
\hline crystal size $[\mathrm{mm}]$ & $0.20 \times 0.20 \times 0.01$ & min./max. transmission & $0.9923 / 0.8611$ \\
\hline temperature $[\mathrm{K}]$ & $100(5)$ & $\theta$ range $\left[{ }^{\circ}\right]$ & $1.05-25.74$ \\
\hline crystal system & monoclinic & completeness to $\theta_{\max }$ & $99.4 \%$ \\
\hline space group & $P 2_{1} / n$ & reflections collected & 134253 \\
\hline$a[\AA]]$ & $9.9910(15)$ & independent reflections & 15050 \\
\hline$b[\AA]$ & $29.253(4)$ & $R_{\text {int }}$ & 0.0532 \\
\hline$c[\AA]$ & $25.866(4)$ & restraints / parameters & $426 / 960$ \\
\hline$\alpha\left[^{\circ}\right]$ & 90 & GooF & 1.127 \\
\hline$\beta\left[^{\circ}\right]$ & $94.831(2)$ & $R 1$ (all data) & 0.0592 \\
\hline$\gamma\left[^{\circ}\right]$ & 90 & $R 1(\mid>2 \sigma(I))$ & 0.0539 \\
\hline$V\left[\AA^{3}\right]$ & $7532.9(19) \AA^{3}$ & $w R 2$ (all data) & 0.1271 \\
\hline Z & 4 & $w R 2(1>2 \sigma(l))$ & 0.1244 \\
\hline$\rho_{\text {calc }}\left[\mathrm{g} \mathrm{cm}^{-3}\right]$ & 1.322 & diff. peak/hole $\left[\mathrm{e}^{-3}\right]$ & $0.810 /-0.452$ \\
\hline
\end{tabular}




\section{References}

[1] J. Deberitz, in Lithium - Production and application of a fascinating and versatile element, Vol. 2, Verlag Moderne Industrie, München, 2006.

[2] M. Schlosser, in Organometallics in Synthesis - A Manual, 2nd Ed. ed. (Ed.: M. Schlosser), John Wiley \& Sons Ltd., Chichester, 2004, pp. 1.

[3] F. A. Carey, R. J. Sundberg, in Organische Chemie, Vol. 2, WILEY-VCH, Weinheim, 2004.

[4] T. Stey, D. Stalke, in The chemistry of organolithium compounds (Eds.: Z. Rappoport, I. Marek), John Wiley \& Sons, Chichester (GB), 2004, pp. 47.

[5] A. Streitwieser, S. M. Bachrach, A. Dorigo, P. v. R. Schleyer, in Lithium Chemistry - A Theoretical and Chemical Overview (Eds.: P. v. R. Schleyer, A.-M. Sapse), John Wiley \& Sons, New York, 1995, pp. 1.

[6] F. M. Bickelhaupt, N. J. R. van Eikema Hommes, C. F. Guerra, E. J. Baerends, Organometallics 1996, 15, 2923.

[7] F. M. Bickelhaupt, M. Solà, C. F. Guerra, J. Chem. Theory Comput. 2006, 2, 965.

[8] E. Matito, J. Poater, F. M. Bickelhaupt, M. Solà, J. Phys. Chem. 2006, B110, 7189.

[9] B. J. Wakefield, in The Chemistry of Organolithium Compounds, Pergamon Press, Oxford, New York, 1974.

[10] V. H. Gessner, C. Däschlein, C. Strohmann, Chem. Eur. J. 2009, 15, 3320.

[11] T. Kottke, D. Stalke, J. Appl. Crystallogr. 1993, 26, 615.

[12] T. Kottke, D. Stalke, Angew. Chem. 1993, 105, 619; Angew. Chem. Int. Ed. Engl. 1993, 32, 580 .

[13] N. D. R. Barnett, R. E. Mulvey, J. Am. Chem. Soc. 1993, 115, 1573.

[14] M. A. Nichols, P. G. Williard, J. Am. Chem. Soc. 1993, 115, 1568.

[15] D. B. Collum, A. J. M. McNeil, A. Ramirez, Angew. Chem. 2007, 119, 3060; Angewandte Chemie Int. Ed. 2007, 46, 3002.

[16] R. A. Gossage, J. T. B. H. Jastrzebski, G. van Koten, Angew. Chem. 2005, 44, 1448; Angew. Chem. Int. Ed 2005, 117, 1472.

[17] D. Seebach, Angew. Chem. 1988, 100, 1685; Angew. Chem. Int. Ed. Engl. 1988, 27, 1624.

[18] B. Tchoubar, A. Loupy, in Salt Effects in Organic and Organometallic Chemistry, Wiley-VCH, New York, 1992.

[19] B. L. Lucht, D. B. Collum, Acc. Chem. Res. 1999, 32, 1035.

[20] F. M. Piller, P. Appukuttan; A. Gavryushin; M. Helm; P Knochel, Angew. Chem. 2008, 120, 6907; Angew. Chem. Int. Ed. 2008, 47, 6802.

[21] C. M. P. Kronenburg, E. Rijnberg, J. T. B. H. Jastrzebski, H. Kooijman, A. L. Spek, G. van Koten, Eur. J. Org. Chem. 2004, 153.

[22] P. Beak, A. I. Meyers, Acc. Chem. Res. 1986, 19, 356. 
[23] V. Snieckus, Chem. Rev. 1990, 90, 879.

[24] C. M. P. Kronenburg, E. Rijnberg, J. T. B. H. Jastrzebski, A. L. Spek, G. van Koten, Chem. Eur. J. 2005, 11, 253.

[25] U. Schöllkopf, W. Hartwig, U. Groth, Angew. Chem. 1979, 91, 922; Angew. Chem. Int. Ed. Engl. 1979, 18, 863.

[26] D. A. Evans, Aldrichimica Acta 1982, 15, 23.

[27] J. R. Gage, D. A. Evans, Org. Synth. 1993, 8, 339.

[28] D. A. Evans, M. D. Ennis, D. J. Mathre, J. Am. Chem. Soc. 1982, 104, 1737.

[29] P. J. Cox, N. S. Simpkins, Tetrahedron: Asymmetry 1991, 2, 1.

[30] P. O'Brien, J. Chem.Com. 1998, Perkin Trans. 1, 1439.

[31] D. Hoppe, T. Hense, Angew. Chem. 1997, 109, 2376; Angew. Chem. Int. Ed. Engl. 1997, 36, 2282.

[32] C. Strohmann, T. Seibel, K. Strohfeldt, Angew. Chem. 2003, 115, 4669; Angew. Chem. Int. Ed. 2003, 42, 4531.

[33] T. Tatic, H. Ott, D. Stalke, Eur. J. Inorg. Chem. 2008, 3765.

[34] D. Hoppe, F. Hintze, P. Tebben, Angew. Chem. 1990, 102, 1457; Angew. Chem. Int. Ed. Engl. 1990, 29, 1422.

[35] S. C. Kuo, F. Chen, D. Hou, A. Kim-Maede, C. Bernard, J. Liu, S. Levy, G. G. Wu, J. Org. Chem. 2002, 68, 4984.

[36] D. Barr, P. R. Raithhy, P. v. R. Schleyer, R. Snaith, D. S. Wright, J., J. Chem. Soc. Chem. Commun. 1990, 643.

[37] C. Lambert, P. v. R. Schleyer, U. Pieper, D. Stalke, Angew. Chem. 1992, 104, 78; Angew. Chem. Int. Ed. Engl. 1992, 31, 77.

[38] F. Xu, R. A. Reamer, R. Tillyer, J. M. Cummins, E. J. J. Grabowski, P. J. Reider, D. B. Collum, J. C. Huffman, J. Am. Chem. Soc. 2000, 122, 11212.

[39] A. Thompson, E. G. Corley, M. F. Huntington, E. J. J. Grabowski, J. F. Reamer, D. B. Collum, J. Am. Chem. Soc. 1998, 120, 2028.

[40] L. Tan, C. Chen, R. Tillyer, E. J. J. Grabowski, P. J. Reider, Angew. Chem. 1999, 111, 724; Angew. Chem. Int. Ed. Engl. 1999, 38, 711.

[41] R. L. Parsons, J. M. Fortunak, J. L. Dorow, G. D. Harris, G. S. Kauffman, W. A. Nugent, M. D. Winemiller, T. F. Briggs, B. Xiang, D. B. Collum, J. Am. Chem. Soc. 2001, 123, 9135.

[42] C. Elschenbroich, in Organometallchemie - Eine kurze Einführung, Vol. 6, Teubner, Stuttgart, 2008.

[43] R. E. Mulvey, F. Mongin, M. Uchiyama, Y. Kondo, Angew. Chem. 2007, 119, 3876; Angew. Chem. Int. Ed. 2007, 46, 3802.

[44] A. Krasovskiy, P. Knochel, Angew. Chem. 2004, 116, 3396; Angew. Chem. Int. Ed. 2004, 43, 3333.

[45] H. Ren, A. Krasovskiy, P. Knochel, Chem. Commun. 2005, 543. 
[46] A. Krasovskiy, V. Krasovskaya, P. Knochel, Angew. Chem. 2006, 118, 511; Angew. Chem. Int. Ed. 2006, 45, 2958.

[47] R. A. Benkeser, D. Goggin, G. Schroll, J. Am. Chem. Soc. 1954, 76, 4025.

[48] M. D. Rausch, D. J. Ciappenelli, J. Organomet. Chem. 1967, 10, 127.

[49] A. F. Halasa, D. P. Tate, J. Organomet. Chem. 1970, 24, 769.

[50] W. Clegg, K. W. Henderson, A. R. Kennedy, R. E. Mulvey, C. T. O'Hara, R. B. Rowlings, D. M. Tooke, Angew. Chem. 2001, 113, 4020; Angew. Chem. Int. Ed. 2001, 40, 3902.

[51] H. Naka, M. Uchiyama, Y. Matsumoto, A. E. H. Wheatley, M. McPartlin, J. V. Morey, Y. Kondo, J. Am. Chem. Soc. 2007, 1921.

[52] Y. Kondo, M. Shilai, M. Uchiyama, T. Sakamoto, J. Am. Chem. Soc. 1999, 121, 3539.

[53] M. Uchiyama, Y. Matsumoto, D. Nobuto, T. Furuyama, K. Yamaguchi, J. Morokuma, J. Am. Chem. Soc. 2006, 128, 8748.

[54] J. Garcia-Alvarez, A. R. Kennedy, J. Klett, R. E. Mulvey, Angew. Chem. 2007, 119, 1123; Angew. Chem. Int. Ed. 2007, 46, 1105.

[55] P. Hohenberg, W. Kohn, Phys. Rev. 1964, B864, 136.

[56] N. K. Hansen, P. Coppens, Acta Crystallogr. 1978, A34, 909.

[57] K. Meindl, J. Henn, N. Kocher, D. Leusser, K. A. Zachariasse, G. M. Sheldrick, T. Koritsanszky, D. Stalke, J. Phys. Chem. A 2009, 113, 9684.

[58] M. J. Frisch, G. W. Trucks, H. B. Schlegel, G. E. Scuseria, M. A. Robb, J. R. Cheeseman, J. A. Montgomery, J. Vreven, T. , K. N. Kudin, J. C. Burant, J. M. Millam, S. S. lyengar, J. Tomasi, V. Barone, B. Mennucci, M. Cossi, G. Scalmani, N. Rega, G. A. Petersson, H. Nakatsuji, M. Hada, M. Ehara, K. Toyota, R. Fukuda, J. Hasegawa, M. Ishida, T. Nakajima, Y. Honda, O. Kitao, H. Nakai, M. Klene, X. Li, J. E. Knox, H. P. Hratchian, J. B. Cross, V. Bakken, C. Adamo, J. Jaramillo, R. Gomperts, R. E. Stratmann, O. Yazyev, A. J. Austin, R. Cammi, C. Pomelli, J. W. Ochterski, P. Y. Ayala, K. Morokuma, G. A. Voth, P. Salvador, J. J. Dannenberg, V. G. Zakrzewski, S. Dapprich, A. D. Daniels, M. C. Strain, O. Farkas, D. K. Malick, A. D. Rabuck, K. Raghavachari, J. B. Foresman, J. V. Ortiz, Q. Cui, A. G. Baboul, S. Clifford, J. Cioslowski, B. B. Stefanov, G. Liu, A. Liashenko, P. Piskorz, I. Komaromi, R. L. Martin, D. J. Fox, T. Keith, M. A. Al-Laham, C. Y. Peng, A. Nanayakkara, M. Challacombe, P. M. W. Gill, B. Johnson, W. Chen, M. W. Wong, C. Gonzalez, J. A. Pople, Gaussian, Inc., Wallingford CT, 2003.

[59] R. Ahlrichs, M. Bär, M. Häser, H. Horn, C. Kölmel, Chem. Phys. Lett. 1989, 162, 165.

[60] O. Treutler, R. Ahlrichs, J. Chem. Phys. 1995, 102, 346.

[61] K. Eichkorn, F. Weigend, O. Treutler, R. Ahlrichs, Theor. Chem. Acc. 1997, 97, 119.

[62] F. Jensen, in Introduction to Computational Chemistry, Wiley, West Sussex, 2007.

[63] W. Kohn, L. Sham, Phys. Rev. 1965, A140, 1133.

[64] A. D. Becke, Phys. Rev. 1988, A38, 3098. 
[65] C. Lee, W. Yang, R. G. Parr, Phys. Rev. 1988, B37, 785.

[66] A. D. Becke, J. Chem. Phys. 1993, 98, 5648.

[67] W. Kohn, Rev. Mod. Phys. 1999, 71, 1253.

[68] G. N. Lewis, J. Am. Chem. Soc. 1916, 38, 762.

[69] S. Shaik, P. C. Hiberty, Rev. Comp. Chem. 2004, $20,1$.

[70] A. E. Reed, R. B. Weinstock, F. Weinhold, J. Chem. Phys. 1985, 83, 735.

[71] F. L. Hirshfeld, Theor. Chim. Acta 1977, 44, 129.

[72] R. S. Mulliken, J. Chem. Phys. 1962, 36, 3428.

[73] R. F. W. Bader, in Atoms in Molecules, Oxford University Press, New York, 1990.

[74] A. D. Becke, K. E. Edgecombe, J. Chem. Phys. 1990, 92, 5397.

[75] M. Kohout, Int. J. Quantum Chem. 2004, 97, 651.

[76] M. Kohout, K. Pernal, F. R. Wagner, Y. Grin, Theor. Chem. Acc. 2004, 112, 453.

[77] P. Popelier, in Atoms in Molecules - An Introduction, Pearson Education Limited, Harlow, 2000.

[78] M. Kohout, DGrid, version 4.4, Germany, Radebeul, 2008.

[79] C. Gatti, P. Fantucci, G. Pacchioni, Theor. Chim. Acta 1987, 72, 433.

[80] R. F. W. Bader, J. Phys. Chem. A 1998, 102, 7314.

[81] S. Deuerlein, D. Leusser, U. Flierler, H. Ott, D. Stalke, Organometallics 2008, 27, 2306.

[82] R. F. W. Bader, H. Essén, J. Chem. Phys. 1984, 80, 1943.

[83] R. F. W. Bader, P. J. MacDougall, J. Am. Chem. Soc. 1985, 107, 6788.

[84] T. S. Koritsanszky, P. Coppens, Chem. Rev. 2001, 101, 1583.

[85] T. Kato, Comm. Pure Appl. Math. 1957, 10, 151.

[86] A. Savin, R. Nesper, S. Wengert, T. F. Fässler, Angew. Chem. 1997, 109, 1892; Angew. Chem. Int. Ed. Engl. 1997, 36, 1808.

[87] H. Gilman, R. L. Bebb, J. Am. Chem. Soc. 1939, 61, 109.

[88] G. Wittig, G. Fuhrman, Chem. Ber. 1940, 73, 1197.

[89] S. T. Chadwick, R. A. Rennels, J. L. Rutherford, D. B. Collum, J. Am. Chem. Soc. 2000, 122, 8640.

[90] J. D. Roberts, D. Y. Curtin, J. Am. Chem. Soc. 1946, 68, 1658.

[91] M. C. Whisler, S. MacNeil, V. Snieckus, P. Beak, Angew. Chem. 2004, 116, 2256; Angew. Chem. Int. Ed. 2004, 43, 2206.

[92] W. Bauer, P. v. R. Schleyer, J. Am. Chem. Soc. 1989, 111, 7191.

[93] S. Harder, J. Boersma, L. Brandsma, G. P. M. van Mier, J. A. Kanters, J. Organomet. Chem. 1989, 364, 1.

[94] S. Harder, J. Boersma, L. Brandsma, J. A. Kanters, J. Organomet. Chem. 1988, 339, 7.

[95] H. Köster, D. Thoennes, E. Weiss, J. Organomet. Chem. 1978, $160,1$. 
[96] Cambridge Structural Database, v5.30 (November 2008), Cambridge Crystallographic Data Centre, Cambridge, 2008.

[97] A. Schäfer, C. Huber, R. Ahlrichs, J. Chem. Phys. 1994, 100, 5829.

[98] J. P. Ritchie, S. M. Bachrach, J. Am. Chem. Soc. 1987, 109, 5909.

[99] R. Ponec, J. Roithova, X. Gironés, L. Lain, A. Torre, R. Bochicchio, J. Phys. Chem. A 2002, 106, 1019.

[100] A. Haaland, D. J. Shorokhov, N. V. Tverdova, Chem. Eur. J. 2003, 10, 4416.

[101] A. M. Pendás, E. Francisco, M. A. Blanco, C. Gatti, Chem. Eur. J. 2007, 13, 9362.

[102] L. Lochmann, J. Pospísil, D. Lím, Tetrahedron Lett. 1966, 2, 257.

[103] M. Schlosser, J. Organomet. Chem. 1967, 8, 9.

[104] M. Schlosser, in Struktur und Reaktivität polarer Organometalle, Springer, Berlin, 1973.

[105] G. van Koten, Pure Appl. Chem. 1989, 61, 1681.

[106] J. F. McGarrity, C. A. Ogle, J. Am. Chem. Soc. 1985, 107, 1805.

[107] M. Marsch, K. Harms, L. Lochmann, G. Boche, Angew. Chem. 1990, 102, 334; Angew. Chem. Int. Ed. Engl. 1990, 29, 308.

[108] L. Lochmann, Eur. J. Inorg. Chem. 2000, 1115.

[109] M. Schlosser, Pure Appl. Chem. 1988, 60, 1627.

[110] W. Bauer, L. Lochmann, J. Am. Chem. Soc. 1992, 114, 7482.

[111] R. E. Mulvey, Chem. Commun. 2001, 1049.

[112] D. R. Armstrong, A. R. Kennedy, R. E. Mulvey, R. B. Rowlings, Angew. Chem. 1999, 111, 231; Angew. Chem. Int. Ed. Engl. 1999, 38, 131.

[113] G. Boche, J. C. W. Lohrenz, Chem. Rev. 2001, 101, 697.

[114] R. E. Mulvey, Chem. Soc. Rev. 1991, 20, 167.

[115] H. Nekola, F. Olbrich, U. Behrens, Z. Anorg. Allg. Chem. 2002, 628, 2067.

[116] J. F. Allan, R. Nassar, E. Specht, A. Beatty, N. Calin, K. W. Henderson, J. Am. Chem. Soc. 2004, 126, 484.

[117] C. Lambert, F. Hampel, P. v. R. Schleyer, M. G. Davidson, R. Snaith, J. Organomet. Chem. 1995, 487, 139.

[118] J. F. Allan, R. Nassar, E. Specht, A. Beatty, N. Calin, K. W. Henderson, J. Am. Chem. Soc. 2004, 484.

[119] H. Dietrich, Acta Crystallogr., Sect. E. 1963, 16, 681.

[120] E. Weiss, E. A. C. Lucken, J. Organomet. Chem. 1964, 2, 197.

[121] M. Adamczyk, D. S. Watt, D. A. Naetzel, J. Org. Chem. 1984, 49, 4226.

[122] Y. Nagaoka, K. Tomioka, J. Org. Chem. 1998, 63, 6428.

[123] A. P. Krapcho, D. S. Kashdan, E. G. E. Jahngen, J. Org. Chem. 1977, 42, 1189.

[124] M. Mallet, G. Quéguiner, Tetrahedron 1982, 38, 3035.

[125] J. L. Herrmann, G. R. Kieczykowski, R. H. Schlessinger, Tetrahedron Lett. 1973, 2433 
[126] P. G. Williard, J. M. Salvino, J. Org. Chem., 58, 1.

[127] D. R. Armstrong, D. Barr, C. W., S. M. Hodgson, R. E. Mulvey, D. Reed, R. Snaith, D. S. Wright, J. Am. Chem. Soc. 1989, 4719.

[128] N. D. R. Barnett, R. E. Mulvey, J. Am. Chem. Soc. 1991, 113, 8187.

[129] D. B. Collum, Acc. Chem. Res. 1992, 25, 448.

[130] J. L. Rutherford, D. Hoffmann, D. B. Collum, J. Am. Chem. Soc. 2002, 124, 264.

[131] M. P. Bernstein, F. E. Romesberg, D. J. Fuller, A. T. Harrison, P. G. Williard, Q. Y. Liu, D. B. Collum, J. Am. Chem. Soc. 1992, 114, 5100.

[132] B. Walfort, L. Lameyer, W. Weiss, R. Herbst-Irmer, R. Bertermann, J. Rocha, D. Stalke, Chem. Eur. J. 2001, 7, 1417.

[133] P. Müller, R. Herbst-Irmer, A. L. Spek, T. R. Schneider, M. R. Sawaya, in IUCr Texts on Crystallography, Vol. 8, 1 ed. (Ed.: P. Müller), Oxford University Press, Oxford (England), 2006.

[134] N. S. Simpkins, Pure Appl. Chem. 1996, 68, 691.

[135] D. Pettersen, M. Amedjkouh, P. Ahlberg, Tetrahedron 2002, 58, 4669.

[136] B. J. Bunn, P. J. Cox, N. S. Simpkins, Tetrahedron 1993, 49, 207.

[137] R. E. Ireland, P. Wipf, J. D. Armstrong, J. Org. Chem. 1991, 56, 650.

[138] M. Majewski, D. M. Gleave, J. Org. Chem. 1992, 57, 1599.

[139] A. J. Edwards, S. Hockey, F. S. Mair, P. R. Raithby, R. Snaith , N. S. Simpkins, J. Org. Chem. 1993, 58, 6942.

[140] P. C. Andrews, P. J. Duggan, M. Maguire, P. J. Nichols, Chem. Commun. 2001, 53.

[141] J. A. Wanklyn, Liebigs Ann. Chem. 1858, 107, 125.

[142] K. Soai, S. Niwa, Chem. Rev. 1992, 833.

[143] E. Negishi, M. Kobayashi, J. Org. Chem. 1980, 45, 5223.

[144] E. Erdik, Tetrahedron 1992, 48, 9557.

[145] E. Nakamura, I. Klawajima, Tetrahedron Lett. 1986, 27, 83.

[146] W. Schlenk, J. Holtz, Ber. Dtsch. Chem. Ges. 1917, 50, 262.

[147] H. Gornitzka, C. Hemmert, G. Bertrand, M. Pfeiffer, D. Stalke, Organometallics 2000, $19,112$.

[148] G. Wittig, F. J. Meyer, G. Lange, Justus Liebigs Ann. Chem. 1951, 571, 167.

[149] H. Gilman, F. K. Cartledge, J. Organomet. Chem. 1964, 2, 447.

[150] T. A. Mobley, S. Berger, Angew. Chem. 1999, 20, 3256; Angew. Chem. Int. Ed. 1999, 20,3070 .

[151] H. Tang, M. Parvez, H. G. Richey, Jr., Organometallics 2000, 19, 4810.

[152] J. F. G. A. Jansen, B. L. Feringa, Tetrahedron Lett. 1988, 29, 3593.

[153] Y. Kondo, N. Takazawa, C. Yamazaki, T. Sakamoto, J. Org. Chem. 1994, 59, 4717.

[154] M. Uchiyama, S. Furumoto, M. Saito, Y. Kondo, T. Sakamoto, J. Am. Chem. Soc. 1997, 119, 11425. 
[155] T. Harada, T. Katsuhira, A. Osada, K. Iwazaki, K. Maejima, A. Oku, J. Am. Chem. Soc. 1996, 118, 11377.

[156] E. Weiss, R. Wolfrum, Chem. Ber. 1968, 101, 35.

[157] T. Furuyama, M. Yonehara, S. Arimoto, M. Kobayashi, Y. Matsumoto, M. Uchiyama, Chem. Eur. J. 2008, 14, 10348.

[158] W. Clegg, S. H. Dale, E. Hevia, G. W. Honeyman, R. E. Mulvey, Angew. Chem. 2006, 118, 2430; Angew. Chem. Int. Ed. 2006, 45, 2370.

[159] P. C. Andrikopoulos, D. R. Armstrong, H. R. L. Barley, W. Clegg, S. H. Dale, E. Hevia, G. W. Honeyman, A. R. Kennedy, R. E. Mulvey, J. Am. Chem. Soc. 2005, 127, 6184.

[160] W. Clegg, S. H. Dale, R. W. Harrington, E. Hevia, G. W. Honeyman, R. E. Mulvey, Angew. Chem. 2006, 118, 2434; Angew. Chem. Int. Ed. 2006, 45, 2374.

[161] Y. Kondo, J. V. Morey, J. C. Morgan, H. Naka, D. Nobuto, P. R. Raithby, M. Uchiyama, A. E. H. Wheatley, J. Am. Chem. Soc. 2007, 129, 12734.

[162] D. R. Armstrong, W. Clegg, S. H. Dale, E. Hevia, L. M. Hogg, G. W. Honeyman, R. E. Mulvey, Angew. Chem. 2006, 118, 3859; Angew. Chem. Int. Ed. 2006, 45, 3775.

[163] P. C. Andrikopoulos, D. R. Armstrong, D. V. Graham, E. Hevia, A. R. Kennedy, R. E. Mulvey, C. T. O'Hara, C. Talmard, Angew. Chem. 2005, 117, 3525; Angew. Chem. Int. Ed. 2005, 44, 3459.

[164] G. Wittig, H. Merkle, Chem. Ber. Dtsch. Chem. Ges. 1942, 75, 1491.

[165] A. R. Lepley, W. A. Khan, A. B. Giumanini, A. G. Giumanini, J. Org. Chem. 1966, 31, 2047.

[166] M. Westerhausen, B. Rademacher, W. Schwarz, Z. Anorg. Allg. Chem. 1993, 619, 675.

[167] M. Westerhausen, M. Wieneke, W. Ponikwar, H. Nöth, W. Schwarz, Organometallics 1998, 17, 1438.

[168] D. R. Armstrong, C. Dougan, D. V. Graham, E. Hevia, A. R. Kennedy, Organometallics 2008, 27, 6063.

[169] H. Barjat, G. A. Morris, S. Smart, A. G. Swanson, S. C. R. Williams, J. Magn. Reson. 1995, B108, 170.

[170] I. Fernández, E. Martínez-Viviente, F. Breher, P. S. Pregosin, Chem. Eur. J. 2005, 11, 1495.

[171] I. Antes, G. Frenking, Organometallics 1995, 14, 4263.

[172] J. P. Perdew, Phys. Rev. 1986, B33, 8822.

[173] P. Macchi, A. Sironi, Coord. Chem. Rev. 2003, 238-239, 383.

[174] L. J. Farrugia, C. Evans, M. Tegel, J. Phys. Chem. A 2006, 110, 7952.

[175] C. Gatti, D. Lasi, Faraday Discuss. 2007, 135, 55.

[176] A. Reisinger, N. Trapp, I. Krossing, S. Altmannshofer, V. Herz, M. Presnitz, W. Scherer, Angew. Chem. 2007, 119, 8445; Angew. Chem. Int. Ed. 2007, 46, 8295. 
[177] U. Flierler, M. Burzler, D. Leusser, J. Henn, H. Ott, H. Braunschweig, D. Stalke, Angew. Chem. 2008, 120, 4393; Angew. Chem. Int. Ed. 2008, 47, 4321.

[178] L. J. Farrugia, C. Evans, D. Lentz, M. Roemer, J. Am. Chem. Soc. 2009, 131, 1251.

[179] N. Bartlett, D. H. Lohmann, J. Chem. Soc. 1962, 5253.

[180] F. A. Cotton, G. Wilkinson, C. A. Murillo, M. Bochmann, in Advanced inorganic chemistry, Vol. 6, John Wiley \& Sons, Inc., New York, 1999.

[181] R. J. Gillespie, Coord. Chem. Rev. 2008, 252, 1315.

[182] W. Kutzelnigg, Angew. Chem. 1984, 96, 262; Angew. Chem. Int. Ed. Engl. 1984, 25, 272.

[183] S. Noury, B. Silvi, R. J. Gillespie, Inorg. Chem. 2002, 41, 2164.

[184] R. J. Gillespie, B. Silvi, Coord. Chem. Rev. 2002, 233-234, 53.

[185] D. G. Gilheany, Chem. Rev. 1994, 94, 1339.

[186] J. A. Dobado, H. Martínez-Garzía, J. M. Molina, M. R. Sundberg, J. Am. Chem. Soc. 1998, 120, 8461.

[187] D. B. Chesnut, J. Am. Chem. Soc. 2003, 107, 4307.

[188] A. Savin, B. Silvi, F. Colonna, Can. J. Chem. 1996, 74, 1088.

[189] G. Jansen, S. Raub, Theor. Chem. Acc. 2001, 106, 223.

[190] O. Glemser, J. Wegener, Angew. Chem. 1970, 82, 324; Angew. Chem. Int. Ed. Engl. 1970, 9, 309.

[191] R. Fleischer, S. Freitag, D. Stalke, J. Chem. Soc., Dalton Trans. 1998, 193.

[192] S. Pohl, B. Krebs, U. Seyer, G. Henkel, Chem. Ber. 1979, 112, 1751.

[193] I. Mayer, J. Mol. Struct. 1987, 149, 81.

[194] D. Leusser, J. Henn, N. Kocher, B. Engels, D. Stalke, J. Am. Chem. Soc. 2004, 126, 1781.

[195] B. Walfort, A. P. Leedham, C. R. Russell, D. Stalke, Inorg. Chem. 2001, 40, 5668.

[196] Bruker AXS Inst. Inc., APEX2 v2.2-0, WI, USA, Madison, 2008.

[197] G. M. Sheldrick, CELL_NOW in Bruker APEX v2.1-0, WI, USA, Madison, 2005.

[198] Bruker AXS Inc., SAINT v7.46A, WI, USA, Madison, 2007.

[199] G. M. Sheldrick, SADABS 2008/2, Göttingen, 2008.

[200] G. M. Sheldrick, TWINABS 2008/1, Göttingen, 2008.

[201] G. M. Sheldrick, XPREP Version 2005/2 for Windows, WI, USA, Madison, 2005.

[202] G. M. Sheldrick, SHELXS in SHELXTL v2008/4, WI, USA, Madison, 2008.

[203] P. v. d. Sluis, A. L. Spek, Acta Crystallogr. 1990, A46, 194. 


\section{LIST OF PUBLICATIONS}

[1] Sebastian Merkel, Daniel Stern, Julian Henn, Dietmar Stalke, "Solvent separated and contact ion pairs of parent lithium trimethyl zincate" Angew. Chem. 2009, 121, 6468; Angew. Chem. Int. Ed. 2009, 48, 6350.

[2] Rajendra S. Ghadwal, Herbert W. Roesky, Sebastian Merkel, Julian Henn, Dietmar Stalke, "Lewis Base Stabilized Dichlorosilylene" Angew. Chem. 2009, 121, 5793; Angew. Chem. Int. Ed. 2009, 48, 5683.

[3] Rajendra S. Ghadwal, Sakya S. Sen, Herbert W. Roesky, Gasper Tavcar, Sebastian Merkel, Dietmar Stalke, "Neutral Penta- and Hexacoordinate N-Heterocyclic Carbene Complexes Derived from SiX 4 (X = F, Br)", Organometallics, 2009, 28 , 6374.

[4] Jianfeng Li, Carola Schulzke, Sebastian Merkel, Herbert W. Roesky, Prinson P. Samuel, Alexander Döring, Dietmar Stalke, "Synthesis and Characterization of Nheterocyclic Carbene Complexes of Titanium(IV) and Titanium(III)", Z. Anorg. Allg. Chem., submitted.

[5] Rajendra S. Ghadwal, Herbert W. Roesky, Sebastian Merkel, Dietmar Stalke, "Ambiphilicity of Dichlorosilylene in a Single Molecule", Chem. Eur. J., in print.

[6] Jianfeng Li, Sebastian Merkel, Julian Henn, Kathrin Meindl, Alexander Döring, Herbert W. Roesky, Rajendra S. Ghadwal, Dietmar Stalke, "Lewis Base Stabilized Dichlorosilylene: A Novel Two Electron o-Donor Ligand", J. Am. Chem. Soc., submitted. 


\section{Poster Presentations}

[1] Sebastian Merkel, Tanja Tatić, Holger Ott, Stephan Deuerlein, Dietmar Stalke, "Solvatisierte Anionen in Alkalimetallkäfigen“, Wissenschaftsforum Chemie 2007 _ GDCh-Jahrestagung, 2007, Ulm, Germany.

[2] Sebastian Merkel, Kathrin Meindl, Julian Henn, Dietmar Stalke, "Comparison of Bonding Descriptors on a Contact Ion Pair of Trimethylzincat and Diphenyl Phosphinic acid“, Workshop "Electron Localizability and Chemical Bonding”, 2009, MPI Dresden, Germany.

\section{INVITED TALKS}

[1] Sebastian Merkel, "Solvensseparierte- und Kontaktionenpaare von Trimethylzinkat", 11. Norddeutsches Doktorandenkolloquium der Anorganischen Chemie, 2008, Burg Warberg, Germany. 


\section{Curriculum vitae}

\section{Persönliche Daten}

geboren am

Familienstand

Staatsangehörigkeit

\section{Ausbildungsdaten}

Schulausbildung

Studium in Schorndorf

ledig

deutsch

$$
\begin{aligned}
& \text { 08/1985 - 06/1989 Besuch der Grundschule Grünsfeld } \\
& \text { 08/1989 - 06/1998 Besuch des Matthias-Grünewald-Gymnasiums in } \\
& \text { Tauberbischofsheim } \\
& \text { 07/1998 - 04/1999 Allgemeiner Wehrdienst } \\
& \text { 05/1999 - 04/2001 Grundstudium Chemie Diplom an der Julius- } \\
& \text { Maximilians-Universität Würzburg } \\
& \text { Mündliche Diplom-Vorprüfung } \\
& \text { Hauptstudium Chemie Diplom an der Julius- } \\
& \text { Maximilians-Universität Würzburg } \\
& \text { 08/2002 - 02/2003 Praktikum bei der Syngenta AG, Basel } \\
& 02 / 2004 \\
& \text { Mündliche Diplomprüfung } \\
& \text { 03/2004-12/2004 Diplomarbeit an der Julius- Maximilians- } \\
& \text { Universität Würzburg im Arbeitskreis von Prof. G } \\
& \text { Bringmann, Thema: „Untersuchungen zur } \\
& \text { Biogenese der Carbazol-Alkaloide“ } \\
& 04 / 2005- \\
& \text { Beginn der Promotion an der Georg-August- } \\
& \text { Universität Göttingen im Arbeitskreis Prof. D. }
\end{aligned}
$$

